DØ Note \#1752

May, 1993

The D $\emptyset$ Intercryostat Detector: Design Considerations, Test Beam Studies and Initial Performance

Terece Louise Geld

25 


\section{THE DØ INTERCRYOSTAT DETECTOR: DESIGN CONSIDERATIONS,}

TEST BEAM STUDIES AND INITIAL PERFORMANCE

by

Terece Louise Geld

A dissertation submitted in partial fulfillment of the requirements for the degree of Doctor of Philosophy

(Physics)

in The University of Michigan

1993

Doctoral Committee:

Professor Homer A. Neal, Chair Associate Professor Ratindranath Akhoury

Associate Research Scientist Robert C. Ball

Professor Dmitri N. Coucouvanis

Professor T. Michael Sanders

Assistant Professor Kaushik De, The University of Texas, Arlington 


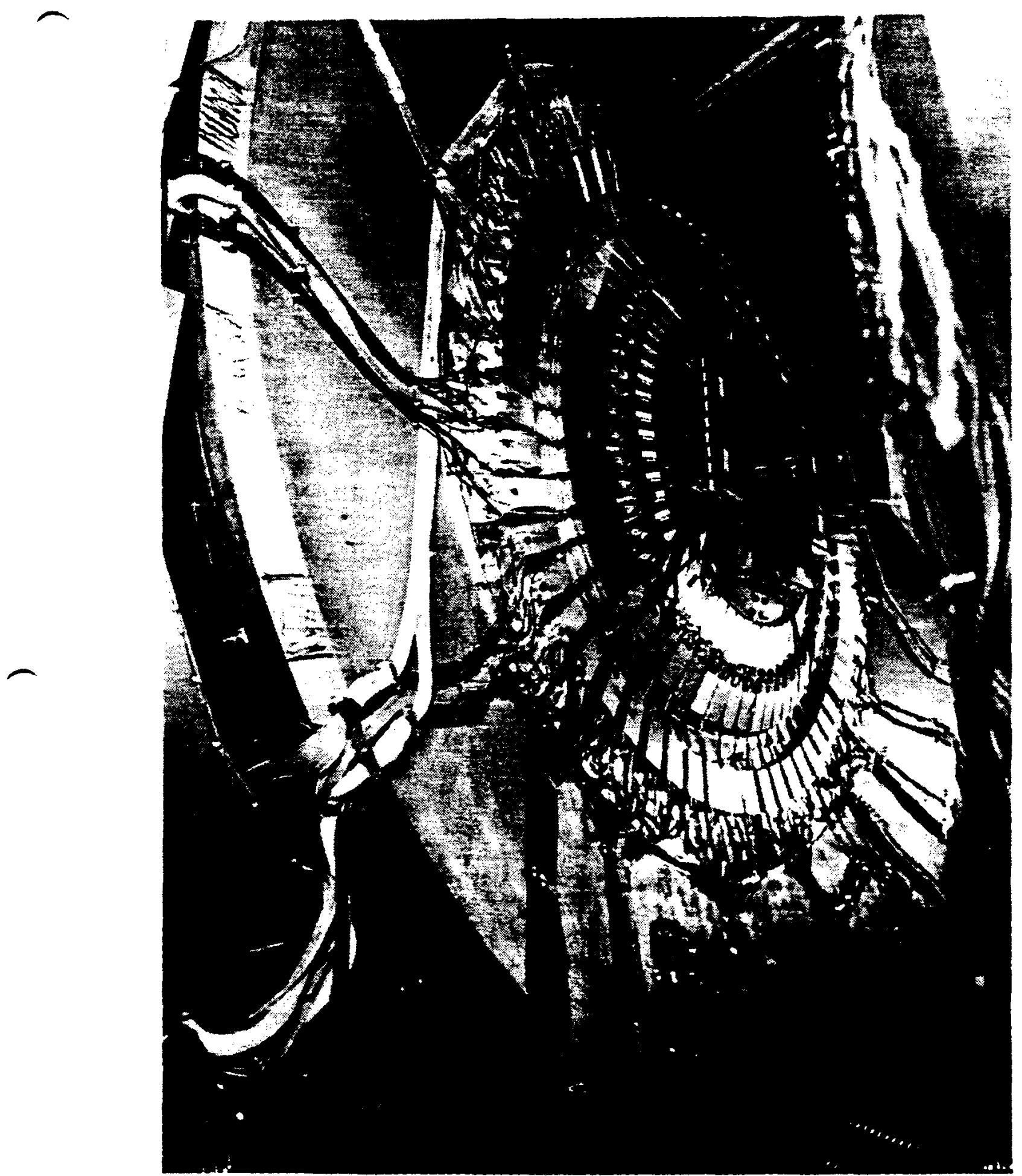


(C) Terece L. Geld 1993

All Rights Reserved 
To my parents ...

who never told me what little giris should not do. 


\section{ACKNOWLEDGEMENTS}

So many people, so few lines. I would like to thank Dr. Homer A. Neal for the guidance and support he has provided me. To Dr. Kaushik De, I owe a debt of gratitude for everything he has done to insure the success of the test beam project and the ICD. A heartfelt thanks

goes out to all the other ICD-er's who have helped make this all possible, but especially, Lori H. Oesch, Peter A. Seegers, David Ciampa, Jeff Youngen, Dr. Andrew P. White, and Dr. Zachary Wolfe.

I would like to thank the other test beam graduate students, especially Terry C. Heuring and John P. Borders, for all their help and solace throughout the test beam run. Thanks go to Jerry Blazey and Bob Hirosky for the contamination studies. Thanks to Bob Ball for the test beam calibration system. Bob and Kaushilk also get gold stars for their more than thorough critique of my writing. My sincere appreciation goes to Mirek Fatyga and Dan Owen for their unwavering help and support in including the ICD in the Test Beam run.

I acknowledge the Department of Energy for its support, without which none of this would have been possible. The $\mathrm{D} \emptyset$ experiment was built by many people at many institutions across the world. Thank you all, for doing such a great job on such a great experiment.

To my husband, Jon, for patience and support and everything else that made this possible. 


\section{TABLE OF CONTENTS}

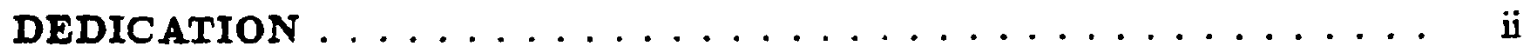

ACKNOWLEDGEMENTS ................

LIST OF TABLES $\ldots \ldots \ldots \ldots \ldots \ldots \ldots \ldots \ldots \ldots$ vi

IIST OF FIGURES $\ldots \ldots \ldots \ldots \ldots \ldots \ldots \ldots \ldots$ vii

LIST OF APPENDICES $\ldots \ldots \ldots \ldots \ldots \ldots \ldots \ldots$ ix

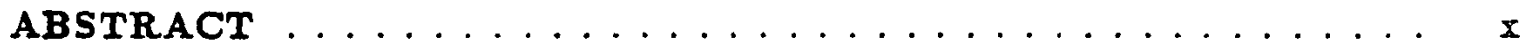

CHAPTER

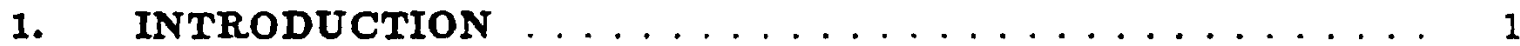

2. THEORY $\ldots \ldots \ldots \ldots \ldots \ldots \ldots \ldots \ldots$

2.1 The Standard Model . . . . . . . . . . . . . . . 4

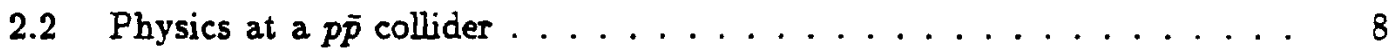

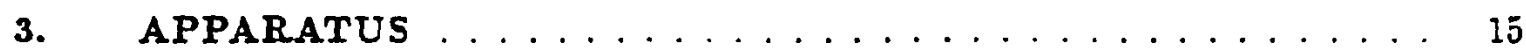

3.1 The Tevatron Collider . . . . . . . . . . . . . . . 15

3.2 The $\mathrm{D} \emptyset$ Detector . . . . . . . . . . . . . . . . . . 19

3.2.1 The Central Tracking Detectors . . . . . . . . . . 20

3.2 .2 The Calorimeters . . . . . . . . . . . . . . 23

3.2 .3 The Muon Detectors . . . . . . . . . . . . . . . . . 29

3.2 .4 The Trigger System . . . . . . . . . . . . . 30

3.3 The Intercryostat Detector . . . . . . . . . . . . . . . 33

3.3.1 Motivation for the Intercryostat Detector . . . . . . . . . . 33

3.3.2 Description of the Intercryostat Detector . . . . . . . . . 35

3.3.3 Intercryostat Detector Electronics . . . . . . . . . . 41

3.3.4 The Laser Calibration System . . . . . . . . . . . . . 43

4. THE TEST BEAM PROJECT $\ldots \ldots \ldots \ldots \ldots \ldots \ldots \ldots$

4.1 Overview of the Test Beam Project . . . . . . . . . . . 45

4.2 The Test Beam Intercryostat Detector . . . . . . . . . . . . . 46

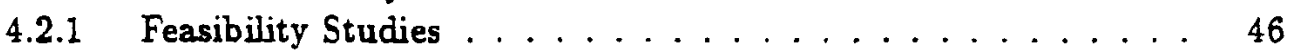


4.2.2 Contamination Studies ................ 63

4.2.3 Final Design ..................... 64

4.2.4 Production of the Test Beam ICD Modules . . . . . . . . . . 65

4.2.5 Calibration System . . . . . . . . . . . . . . . . 66

4.3 Description of Test Beam Setup . . . . . . . . . . . . . . 66

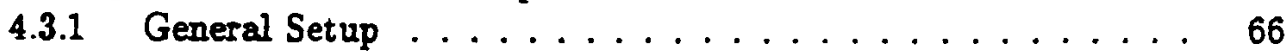

4.3.2 Intercryostat Region Setup . . . . . . . . . . . . 70

4.3.3 Beamline Elements . . . . . . . . . . . . . . . 72

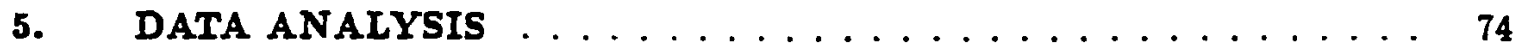

5.1 The ICD Test Beam Mini-DST Package . . . . . . . . . . . . . 74

5.2 Discussion of Energy Loss in Matter . . . . . . . . . . . . . 75

5.3 Muon Calibration of Test Beam Tiles . . . . . . . . . . . . . 76

5.4 Results from Studies with Muon Beams ... . . . . . . . . . . 80

5.4.1 Energy Dependence of MIP Calibration . . . . . . . . . . 81

5.4.2 Stability of Detector Response .............. 83

5.5 Determination of Sampling Fractions .............. 86

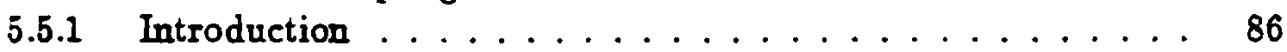

5.5.2 $\chi^{2}$ Optimization Technique .............. 87

5.6 ICD Performance Results . . . . . . . . . . . . . . . 90

5.6.1 Total Energy Determination ............... 90

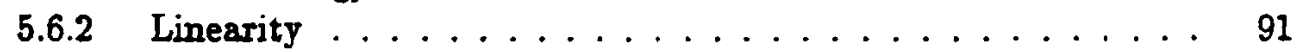

6. THE TEST BEAM MONTE CARLO SIMULATION $\ldots \ldots \ldots$

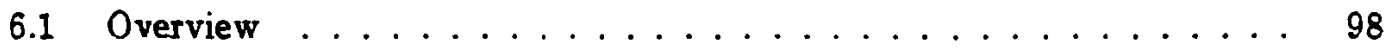

6.2 Description of General Simulation . . . . . . . . . . . . . . . . 98

6.2.1 Description of Calorimeter Simulation . . . . . . . . . . 99

6.2.2 Description of Intercryostat Region Simulation . . . . . . . . . 99

6.3 Determination of Calorimeter Normalization Constants . . . . . . . 102

6.4 Comparison of Monte Carlo Data with Test Beam Data . . . . . . . . 104

7. THE IMPACT OF THE INTERCRYOSTAT DETECTOR ON DO

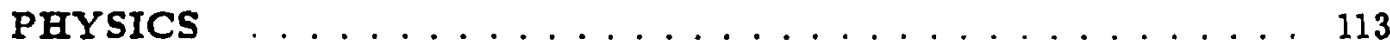

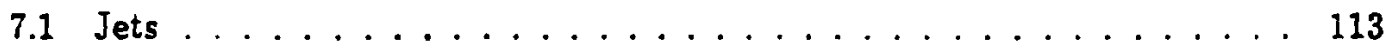

7.2 Jet-Photon Asymmetry . . . . . . . . . . . . . . 115

7.3 Top Search .......................... 116

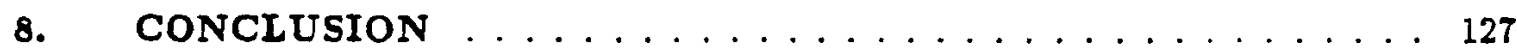

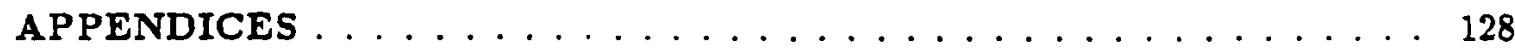

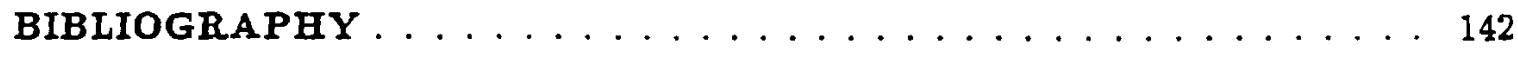




\section{LIST OF TABLES}

\section{Table}

3.1

4.1

4.2

4.3

5.1

5.2

The ICD tile sizes . . . . . . . . . . . . . . . . . . 37

A comparison of DØ ICD and TB ICD design specifications ..... 65

Test beam tile response to cosmic rays . . . . . . . . . . . . 66

The output signal ganging scheme used for the test beam tiles . . . . . 67

Comparison of Landau and Landau + Gaussian fits . . . . . . . . . 79

The test beam tile MIP normalization constants . . . . . . . . . . 80

A comparison of the tile response to different muon energies . . . . . 84

Sampling fraction calculation example .............. 87

Optimized sampling weights in the intercryostat region . . . . . . . 90 The $d E / d x$ sampling fractions used for the test beam Monte Carlo simulation modules . . . . . . . . . . . . . . . . . . 101

The detector readout layer labels . . . . . . . . . . . . . 105

A comparison of the $D \emptyset$ and CDF detectors' $Z_{T}$ resolution . . . . 114 


\section{LIST OF FIGURES}

\begin{tabular}{|c|c|c|}
\hline & & \\
\hline 2.1 & The particles of the Standard Model & 4 \\
\hline 2.2 & A simple hard scattering process & - \\
\hline 2.3 & First order Feynman diagrams for parton-parton scattering & 10 \\
\hline 2.4 & Lowest order Feynman diagrams for direct photon production . . . & 11 \\
\hline 2.5 & Lowest order Feynman diagrams for $Z^{0}$ and $W^{ \pm}$decay $\ldots$. & 11 \\
\hline 2.6 & Decay channels for top production & 13 \\
\hline 2.7 & Higgs production via $\mathrm{Z}^{0}$ and $\mathrm{W}^{ \pm}$ & 14 \\
\hline 3.1 & The layout of FNAL's Tevatron and fixed target areas . . . . . . . & 17 \\
\hline 3.2 & A cutaway view of the Central Detector systems & 21 \\
\hline 3.3 & An overview of the $\mathrm{D} \emptyset$ calorimeters & 24 \\
\hline 3.4 & A schematic of the basic calorimeter cell . . . . . . . . . . & 25 \\
\hline 3.5 & An overview of the Level 0 Detector & 32 \\
\hline 3.6 & The fractional energy loss in the intercryostat region & 33 \\
\hline 3.7 & A close-up view of the $\mathrm{D} \emptyset$ calorimeters . . . . . . . . . . & 34 \\
\hline 3.8 & The final design for an ICD tile . . . . & 36 \\
\hline 3.9 & $\ldots \ldots \ldots \ldots \ldots$ & 38 \\
\hline 3.10 & An end-on view of the ICD boxes mounted on the EC cryostat .. & 39 \\
\hline 3.11 & A picture of the installed ICD $\ldots \ldots \ldots \ldots$ & 40 \\
\hline 3.12 & The ICD electronics card design . . . . . . . . . . . . . . . & 42 \\
\hline 4.1 & A schematic of the setup used in fiber splicing . . & 51 \\
\hline 4.2 & A schematic of the liquid nitrogen test setup . . . . . . . . . & 53 \\
\hline 4.3 & PMMA/FP radius of curvature test in air, using UV light & 54 \\
\hline 4.4 & PS/PMMA radius of curvature test in air, using UV light $\ldots \ldots \ldots$ & $5 \overline{5}$ \\
\hline 4.5 & Baseline test of PMMA/FP fiber in air, using green LED . . . . . . . . & 56 \\
\hline 4.6 & PMMA/FP radius of curvature test in air, using green LED . . . & 56 \\
\hline 4.7 & PMMA/FP radius of curvature test in $L N_{2}$, using green LED & 57 \\
\hline 4.8 & PMMA / FP long term radius of curvature test, in $\mathrm{LN}_{2}$, using green LED & 58 \\
\hline 4.9 & A schematic of the setup for the prototype test beam tile test $\ldots \ldots$ & 59 \\
\hline 4.10 & Results from a test beam tile prototype study & 60 \\
\hline 4.11 & Interface between the tile and outer medium . & 62 \\
\hline 4.12 & A plan view of the test beam load & 68 \\
\hline 4.13 & An elevation view of the test beam load & 69 \\
\hline 4.14 & A close-up view of the test beam intercryostat region . . & 71 \\
\hline 4.15 & The Neutrino West Area beamline & 73 \\
\hline 5.1 & Some typical distributions of ADC counts for a muon run & 77 \\
\hline 5.2 & Ratio of Landau peak to Landau + Gaussian peak . . . . & 79 \\
\hline 5.3 & Comparison of test beam tile response to various energies & 82 \\
\hline
\end{tabular}


5.4 Time dependent comparison of test beam tile energy response . . . . 83

5.5 Comparison of test beam tile response to same energ . . . . . . . 84

$5.6 \quad$ Stability of test beam tile response over time . . . . . . . . . 85

5.7 Total energy in intercryostat region, low $\eta^{\prime}$ 's . . . . . . . . . . . 92

5.8 Total energy in intercryostat region, high $\eta^{\prime}$ ' . . . . . . . . . . 93

$5.9 \quad$ Calorimeter energy and resolution . . . . . . . . . . . . 94

$5.10 \quad$ Linearity for $\eta=0.75, \phi=31.6 \ldots \ldots \ldots \ldots$

$5.11 \quad$ Linearity for $\eta=0.85, \phi=31.6 \ldots \ldots \ldots \ldots$

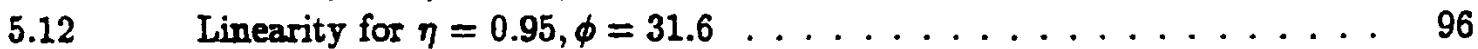

$5.13 \quad$ Linearity for $\eta=1.05, \phi=31.6 \ldots \ldots \ldots 6$

$5.14 \quad$ Linearity for $\eta=1.15, \phi=31.6 \ldots \ldots \ldots . \ldots \ldots$

6.1 Test beam Monte Carlo simulation geometry . . . . . . . . . . 100

6.2 Close-up of intercryostat region in the Monte Carlo simulation geometry 103

6.3 Comparison of test beam data with Monte Carlo simulation data at

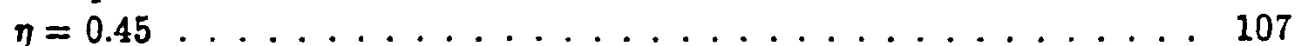

6.4 Comparison of test beam data with Monte Carlo simulation data at

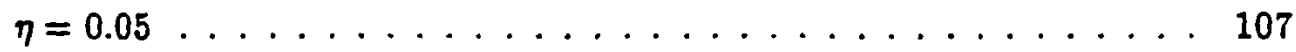

6.5 Comparison of test beam data with Monte Carlo simulation data at $\eta=0.75 \ldots \ldots \ldots \ldots \ldots . \ldots \ldots \ldots$

6.6 Comparison of test beam data with Monte Carlo simulation data at $\eta=0.85 \ldots \ldots \ldots \ldots . \ldots \ldots . \ldots \ldots$

6.7 Comparison of test beam data with Monte Carlo simulation data at

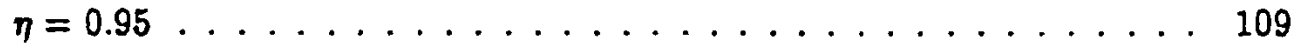

6.8 Comparison of test beam data with Monte Carlo simulation data at

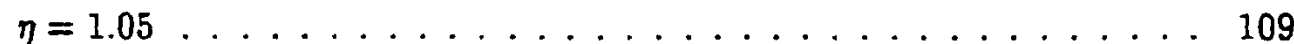

6.9 Comparison of test beam data with Monte Carlo simulation data at

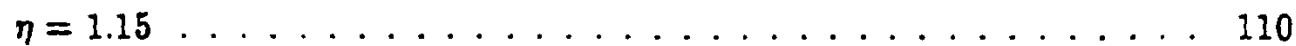

6.10 Comparison of test beam data with Monte Carlo simulation data at

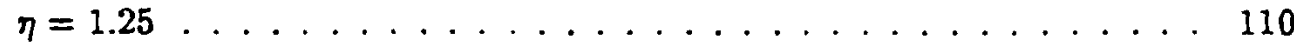

6.11 Comparison of test beam data with Monte Carlo simulation data at

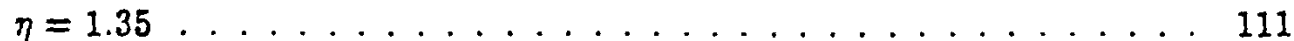

6.12 Comparison of test beam calorimeter energy and resolution with Monte Carlo simulation calorimeter . . . . . . . . . . . . . . . . . 112

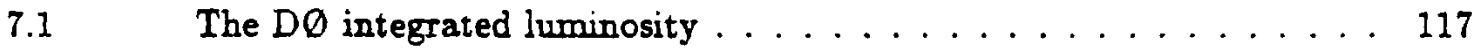

7.2 Event display showing a jet in intercryostat region, R-Z view ... 118

7.3

7.4 Event display showing a jet in intercryostat region, R- $\phi$ view . . . . 119

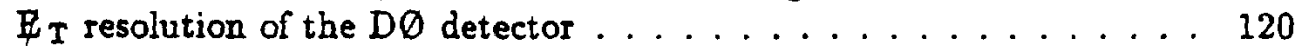
Inclusive cross section for jet production . . . . . . . . . . . . 121 Jet production as a function of $E_{\mathrm{T}} \ldots \ldots \ldots \ldots \ldots \ldots$ Direct photon candidate . . . . . . . . . . . . . . . 123 Asymmetry of jet-photon events . . . . . . . . . . . . . 124 Event display showing a possible top candidate, calorimeter and rauon chambers ........................ 125

7.10 Event display showing a possible top candidate, calorimeter only . . 126 


\section{LIST OF APPENDICES}

\section{Appendix}

A. The Dø Collaboration . . . . . . . . . . . . . . 129

B. Study of inclusive $\Lambda$ production in $e^{+} e^{-}$annihilations at $29 \mathrm{GeV} \ldots 133$

C. Frequently Used Abbreviations . . . . . . . . . . . . . . 140 


\title{
ABSTRACT \\ THE DØ INTERCRYOSTAT DETECTOR: DESIGN CONSIDERATIONS, TEST BEAM STUDIES AND INITIAL PERFORMANCE
}

by

\author{
Terece Louise Geld
}

Chair: Homer A. Neal

Reported herein are the deveiopment, test beam studies and initial performance results of a novel scientific device, the Intercryostat Detector (ICD). The ICD is a subsystem in the $D \emptyset$ Experiment, a major high energy physics experiment running at the Fermi National Accelerator Laboratory's pp Tevatron collider. The D $\emptyset$ detector is designed to study fundamental particle interactions at a center-of-mass energy of $1.8 \mathrm{TeV}$. The ICD plays an integral role in these studies by significantly improving the ability of $D \emptyset$ to measure the energy of particle showers in a critical region of the detector. The ICD uses a layer of scintillator to sample particle showers as they pass through the detector. Its readout system is a unique design of wavelength shifting fiber bundles embedded in the scintillator, which we specifically developed for use in the ICD.

During its development, we studied the ICD in a test beam. The inclusion of the Intercryostat Detector into the test beam run represents the first use of a scintillator sampling device operating in a liquid argon environment. The feasibility studies performed to develop a modified version of the ICD to operate in liquid argon are discussed. The test beam 
project was critical to fully exploit the ability of the ICD to improve the DØ detector performance. The specific calibration constants for the intercryostat detectors, used to relate the detector response to the actual energy deposition in the detector, are determined from the test beam data and are presented. In addition, comparisons are made to Monte Carlo simulation data and the impact of the ICD on the $D \emptyset$ detector performance is discussed. 


\section{CHAPTER 1}

\section{INTRODUCTION}

This dissertation reports on the research and development, design, performance studies, and physies impact of the $D \emptyset$ Intercryostat Detector (ICD). The major portion of this dissertation will focus on the performance studies of the ICD. These performance studies

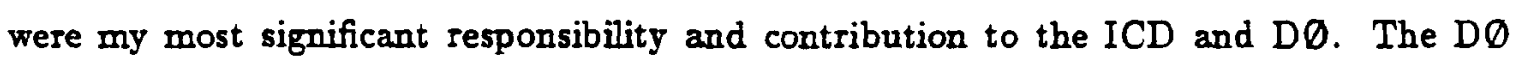
Collaboration is an international collaboration consisting of over 30 institutions, including the University of Michigan. The institutions and collaborators are listed in Appendix A. The focus of the collaboration is the $\mathrm{D} \emptyset$ detector, a major high energy physics detector which is currently running in the $p \bar{p}$ Tevatron collider at Fermi National Accelerator Laboratory (FNAL). The DO detector is designed to study properties of particle interactions at $1.8 \mathrm{TeV}$ center of mass energy. The collaboration and detector derive their name from the Tevatron interaction site, $D 0$, where the $D \emptyset$ detector is located.

The Intercryostat Detector (ICD) is an innovative detector designed to improve the overall solid angle coverage (or 'hermeticity') of the $D O$ detector. The general concept behind the ICD is to use scintillator to sample particle showers as they travel between two large cryostats. The ICD uses a unique readout system of bundles of wavelength shifting optical fibers. A modified version of the ICD was implemented into the final $D \emptyset$ test beam run. This run was carried out in the Neutrino West Area of FNAL's fixed target beam. The successful inclusion of the ICD into this test beam run provided invaluable calibration information for the ICD. Moreover, it was one of the first instances of operating a scintillator based detector in a liquid argon (IAr) environment.

Chapter 2 will discuss the physics background and motivations behind the $D \emptyset$ experiment. The D $\emptyset$ detector and the ICD will be described in detail in Chapter 3. To begin, I would like to give the reader a brief overview of $D \emptyset$, the ICD and my involvement in 
the project. The $D \emptyset$ calorimeter uses liquid argon calorimetry to sample particle showers produced in the $p \bar{p}$ collisions. The calorimeter is contained in three separate cryostats, one central cryostat and two others which cap the central cryostat on either end. The cryostat walls create large amounts of dead material which severely degrade the energy measurement in the gaps between the cryostats. Two separate solutions were proposed to solve this energy measurement problem. One was to include an extra layer of liquid argon sampling, called 'massless gaps' (MG's); the other was to instrument the region between the cryostats with an extra layer of sampling, the Intercryostat Detector.

The University of Michigan and the University of Florida undertook responsibility for the research and development of the ICD. I joined the UM DØ group in early 1988, when the group was just starting. I took part in much of the early prototype work. This included construction and testing of various scintillator and fiber tile designs; testing of various photomultiplier tubes; prototype module designs; prototype calibration system design and the many other aspects. which went into the development of a final ICD design.

In early 1990, one of our DO collaborators suggested it might be possible to include the ICD in the upcoming test beam run. The ICD group had not previously considered the possibility of running in the test beam as it would have required putting the scintillator in liquid argon. The test beam project will discussed in detail in Chapter 4. The test beam offered us the possibility to calibrate the detector in full $\mathrm{DV}$ configuration and to extract the calibration constants needed to use the ICD in DØ. So two of us in the UM group [1] began work on the test beam project. The test beam project itself was an enormous effort carried out by a large number of $D \emptyset$ collaborators. My contributions to the ICD part of the test beam project span all aspects of the project. I worked on the development of the modified ICD design which was used in the test beam; I designed and completed the feasibility studies, described in Chapter 4, to insure that a scintillator-based detector would operate in liquid argon; I worked in collaboration with the Brookhaven group responsible for the overall test beam design to fit the ICD into its small niche in the test cryostat; I built the test beam tiles (with invaluable help from the our group's technical person and work-study students) and installed them in the test beam cryostat (with much help from many $D \emptyset$ collaborators).

Chapter 5 will discuss the test beam data analysis. All the analyses were aided by several software developments, including the DST package which was a responsibility of mine. I was involved in all aspects of the data analysis, including the initial data-taking phase; the 
determination of the calibration constants; the detector normalization necessary to transfer that information from the test beam detector to the real Intercryostat Detector; and the studies of detector stability.

Chapter 6 will describe the test beam Monte Carlo (MC) simulation and its comparison to the test beam data. The Monte Carlo simulation was implemented by many $\mathrm{D} \emptyset$ collaborators. I was responsible for implementing the intercryostat region geometry in the $\mathrm{MC}$, including the ICD, the MG's, and the various dead material in that region; I also wrote the necessary routines to read out the MC energy for these detectors.

Chapter 7 will discuss the impact the ICD has on the D $\emptyset$ detector performance and on the $D \emptyset$ physics. In this chapter, I will be showing some preliminary results from the actual $D \emptyset$ sun, results which are the work of the entire collaboration. Chapter 8 will summarize this thesis. 


\section{CHAPTER 2}

\section{THEORY}

\subsection{The Standard Model}

The basic tenets of particle interactions have evolved over the past three decades and have culminated in the Standard Model (SM) [2, 3, 4, 5]. The SM encompasses both the basic building blocks and forces of nature. The lowest order building blocks consist of two leptons (one charged, one neutral) and two quarks. This scheme is repeated for a total of three generations of quarks and leptons. The three generations are shown in Figure 2.1. The leptons are, by generation, the electron $\left(e^{-}\right)$and its neutrino $\left(\nu_{e}\right)$; the muon $\left(\mu^{-}\right)$and its neutrino $\left(\nu_{\mu}\right) ;$ and the tau $\left(\tau^{-}\right)$and its neutrino $\left(\nu_{\tau}\right)$. The quarks are, by generation, the up $(u)$ and down $(d)$; the charm (c) and strange $(s)$; and the top $(t)$ and bottom $(b)$.

$$
\begin{aligned}
& \text { Quarks : } \quad\left(\begin{array}{l}
u \\
d
\end{array}\right) \quad\left(\begin{array}{l}
c \\
s
\end{array}\right) \quad\left(\begin{array}{l}
t \\
b
\end{array}\right) \\
& \text { Leptons: } \quad\left(\begin{array}{c}
\nu_{e} \\
e
\end{array}\right) \quad\left(\begin{array}{c}
\nu_{\mu} \\
\mu
\end{array}\right) \quad\left(\begin{array}{c}
\nu_{\tau} \\
\tau
\end{array}\right)
\end{aligned}
$$

Figure 2.1: The particles of the Standard Model. There are three generations of quariss and leptons. Currently, the top quark and the tau neutrino have not been confirmed.

There are three forces which govern the interactions between particles in the SM. These forces can all be described by gauge theories and are propagated by spin-one gauge bosons 
$[6,7]$. The electromagnetic force is carried by the neutral photon, $\gamma$; the weak force is carried by one neutral and two charged bosons, $Z^{0}$ and $W^{ \pm}$; and the strong force is carried by eight gluons, $g$.

The Standard Model Lagrangian which describes the interactions between the fermions and gauge bosons is given by [7]:

$$
\begin{aligned}
\mathcal{L} & =\sum_{f=f \text { ermions }} e Q_{f}\left(\bar{f} \gamma^{\mu} f\right) A_{\mu} \\
& +\frac{e}{\sin \theta_{w} \cos \theta_{w}} \sum_{f}\left[\overline{f_{L}} \gamma^{\mu} f_{L}\left(T_{f}^{3}-Q_{f} \sin ^{2} \theta_{w}\right)+\bar{f}_{R} \gamma f_{R}\left(-Q_{f} \sin ^{2} \theta_{w}\right)\right] Z_{\mu} \\
& +\frac{e}{\sqrt{2} \sin \theta_{w}}\left[\left(\overline{u_{L}} \gamma^{\mu} d_{L}+\overline{\nu_{L}} \gamma^{\mu} \nu_{L}\right) W_{\mu}^{+}+\left(\overline{d_{L}} \gamma^{\mu} u_{L}+\bar{e}_{L} \gamma^{\mu} \nu_{L}\right) W_{\mu}^{-}\right] \\
& +\sqrt{\pi \alpha_{,}} \sum_{q=u_{d}} \overline{q_{\alpha}} \gamma^{\mu} \lambda_{\alpha \beta}^{a} q_{\beta} G_{\mu}^{a} \\
& + \text { terms for the second and third generations. }
\end{aligned}
$$

where $Q_{f}$ is the electric charge of the fermion, $T_{3}^{f}$ is the weak isospin $\left(T_{3}^{f}=1 / 2\right.$ for the $\nu_{L}, u_{L} ; 0$ for the $u_{R}, d_{R}$; and $-1 / 2$ for the $\left.e_{L}, d_{L}\right), \theta_{w}$ is the electroweak mixing angle and $\alpha_{,}$is the strong coupling constant. $A_{\mu}$ is the photon field, $Z_{\mu}$ is the $Z^{0}$ boson, $W_{\mu}$ is the $\mathrm{W}^{ \pm}$boson and the $G^{a}$ are the eight gluons. The $\gamma$ and $\lambda$ matrices are the generators of the $S U(2)$ and $S U(3)$ symmetries.

The first term in the Lagrangian is the usual electromagnetic interaction. The second term is the neutral current part of the electroweak interaction, proceeding via the neutral gauge boson, $\mathrm{Z}^{0}$. The third term represents charged current electroweak interactions which proceed via the charged bosons, $\mathrm{W}^{ \pm}$. The fourth term is the strong interaction term.

Electromagnetism is well described by quantum electrodynamics (QED). The beauty of QED is that it is a renormalizable local gauge theory. Renormalizable means that troublesome unphysical infinities in the theory all cancel leaving only finite results. The local gauge means that the Lagrangian is unchanged by a transformation of the type:

$$
\begin{aligned}
\psi(x) & \rightarrow \exp [\operatorname{ie\Lambda }(x)] \psi(x) \\
A_{\mu}(x) & \rightarrow A_{\mu}(x)+\partial \Lambda(x) / \partial x_{\mu}
\end{aligned}
$$

where $\psi(x)$ is the fermion field and $A_{\mu}(x)$ is the photon field. Thus, changing the fermion field and photon field with the same phase factor does not alter the theory. QED is governed by the $U(1)$ symmetry group and the $\exp [i \Lambda(z)]$ are $U(1)$ unitary transforms. The unification of electromagnetism with the theory of weak interactions occurs by combining 
the $U(1)$ QED theory with the $S U(2)$ symmetry which governs weak interactions. The masses of the electroweak gauge bosons, $Z^{0}$ and $W^{ \pm}$, arise from the spontaneous breaking of the $S U(2) \times U(1)$ symmetry group which governs the electroweak interaction.

The gauge theory which describes strong interactions is known as quantum chromodynamics (QCD). Strongly interacting particles transform invariantly under an $S U(3)$ symmetry. All strongly interacting particles carry the QCD quantum number, the color charge. This includes the quarks and the gauge bosons, the gluons. As the propagator of the force also carries the charge, it can have self-interactions. This feature makes QCD a much more complicated theory than QED. As such, QCD can only be used at high energies where the coupling constant, $\alpha_{1}$ is small enough to allow a perturbative treatment. The final states of quarks and gluons (collectively called partons) are color-neutral, which only adds to the complications of QCD. Thus, it is difficult to test QCD directly.

Tests of QCD have used several different types of experiments, such as deep inelastic scattering experiments (DIS), $e^{\dagger} e^{-}$collisions, and hadron-hadron collisions. Consider the simplest case of parton-parton hard scattering as shown in Figure 2.2. The initial states are the hadrons, $A$ and $B$. The final states are two jets of hadrons, with leading hadrons $h_{1}$ and $h_{2}$. In the collision, parton $a$ in hadron $A$, collides with parton $b$ in hadron $B$. The parton momentum is given by the distribution function, $G$, where $G_{a / A(x)}$ is the probability of finding parton $a$ in hadron $A$ with a momentum fraction between $x$ and $x+d x$ of the parent momentum.

In the final states, the outcomes are described by the fragmentation functions, D. These functions give the probability for a final state hadron $h_{1(2)}$ to be produced from parton c (d), with momentum fraction between $z$ and $z+d z$. The parton hard scattering process itself takes place with a cross section of $d \sigma / d t$, which can be calculated from the appropriate lowest order Feynman diagrams.

The process can therefore be divided into two distinct sub-processes. The initial collision takes place subject to the distribution functions of the initial hadrons. The final state hadrons are produced according to the fragmentation functions. DIS and $e^{+} e^{-}$collisions can be used to separate and study individually these two processes. In DIS, an electron (or other lepton) is shot at fixed targets, such as heavy nuclei or protons (hydrogen). The electron acts as a probe to study the substructure, or distribution function, of the protons and neutrons in the target.

In $e^{+} e^{-}$collisions, the colliding particles have no (known) substructure. Therefore, the 


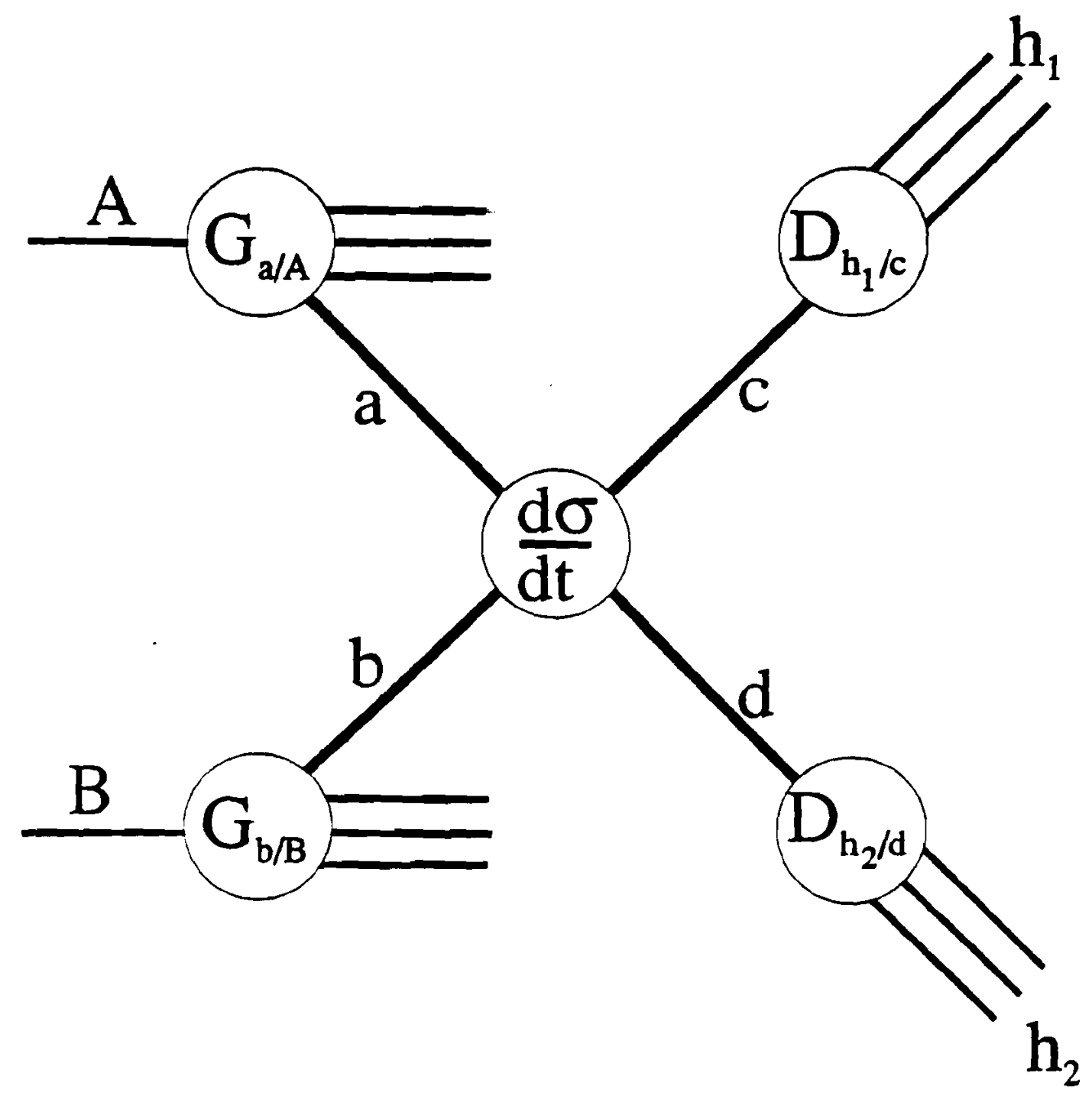

Figure 2.2: A simple hard scattering process for a parton-parton collision. $\mathrm{G}_{a / A(x)}$ and $\mathrm{G}_{b / B(x)}$ are the structure functions for partons $a$ and $b$ in hadrons $A$ and $B ; D_{h_{1} / c}$ and $D_{h_{2} / d}$ are the fragmentation functions for produce hadron 1 or 2 from parton $c$ or d. 
distribution functions are equal to unity. However, the fragmentation process is assumed to proceed according to the same final state fragmentation functions. In a hadron event resulting from an $e^{+} e^{-}$collision, a $q \bar{q}$ pair is immediately formed. The quark pair then fragments into two or more hadrons, which appear as jets of particles.

This fragmentation process is a complicated many-body problem which is incompletely understood. There are currently several theoretical models in use to describe the process. One can hope to learn more about this fragmentation process by studying the decay products from $e^{+} e^{-}$collisions and comparing experimentally determined distributions and cross sections with theoretical predictions. For instance, strange particle production provides a unique opportunity to tag the initial quarks, which helps in understanding the outcome of a collision. Before working on the ICD, I completed a study on strange particle production at the High Resolution Spectrometer [8]. In that study, we determined the cross section for inclusive $\Lambda$ production as a function of the fractional energy and of the momentum of the $\Lambda$. We then compared the experimental distributions with theoretical predictions and used the data to extract a value for $\delta$, the extra strange diquark suppression parameter. This parameter is defined to be:

$$
\delta=\frac{u s / u d}{s / d}
$$

and is a measure of how much more frequently a strange diquark is produced than a nonstrange diquark, normalized to how much more frequently a single strange quark is produced than a single non-strange quark. The $\Lambda$ consists of an up, a down and a strange quark and is thus very sensitive to $\delta$. From our analysis, we determined a value of $\delta=0.59 \pm 0.10$, as compared to the default value of 0.32 , indicating a higher than theoretically expected production of strange particles. I refer the reader to Appendix B, the published paper resulting from that study, Study of inclusive $\Lambda$ production in $e^{+} e^{-}$annihilations at $29 \mathrm{GeV}$ [9].

\subsection{Physics at a $p \bar{p}$ collider}

A detector such as $\mathrm{D} \emptyset$, operating at a high center-of-mass energy, provides an excellent opportunity to test QCD, to further ow understanding of the Standard Model and to probe for new physics beyond the Standard Model. The simplest process is parton-parton scattering, which occurs according to the Feynman diagrams shown in Figure 2.3 [10]. 
Within the detector, the signature of such an event will be two or more high PT hadronic jets plus two low pr beam jets at high rapidity (close to the beam pipe). Additional jets can be created via the radiation of a gluon in the initial and/or final state. The cross-section for inclusive production of jets as a function of jet energy, momentum or other variables can provide a comparison to test theoretical predictions based on the many different structure functions.

From the perspective of detector design, good jet energy measurement and resolution is crucial to the study of jet production, and to many other aspects of QCD and the SM. Equally important is to minimize cracks or gaps in the detector which would degrade the resolution or increase the missing energy (i.e., the energy which is not contained and measured in the calorimeter. The major impetus behind the D $\emptyset$ Intercryostat Detector, as will be discussed in detail in the next chapter, was to fill in a gap between the calorimeter cryostats with an extra layer of energy sampling. In doing so, the total energy measurement and energy resolution is improved and the determination of the missing energy is more accurate.

Direct photon production, $p \bar{p} \rightarrow \gamma+\mathrm{X}$ ( $\mathrm{X}$ is anything), provides another method to test $Q C D[11,12]$. Direct photon production proceeds, in leading order, via the $Q C D$ Compton process, $q g \rightarrow q \gamma$, and the $q \bar{q}$ annihilation process, $q \bar{q} \rightarrow g \gamma$, as shown in the Feynman diagrams in Figure 2.4. The initial gluon in the dominant process $(q g \rightarrow \gamma q)$ provides an excellent method to probe the gluon structure function. Good electromagnetic energy measurement and photon identification are critical to any direct photon studies. An electron or $\pi^{0} \rightarrow 2 \gamma$ can easily mimic a single photon. Good tracking can be used to veto such backgrounds. Since the direct photon is usually balanced by a single jet, direct photon production can be used to check the electromagnetic and hadronic energy scales of a detector.

$D \emptyset$ provides an excellent tool for studying the properties of the the three intermediate vector bosons, the $\mathrm{Z}^{0}$ and the $\mathrm{W}^{ \pm}$. These bosons decay through the diagrams in Figure 2.5 . The signature of a $Z^{0}$ in the detector will be two back-to-back leptons or two back-to-back jets. Good lepton identification and resolution is essential to a good measurement of the $\mathrm{Z}^{0}$ mass and width. The $\mathrm{W}^{ \pm}$signature will be a single lepton plus missing transverse energy $\left(Z_{T}\right)$ or two jets. Once again, good lepton measurement is important, and good $Z_{T}$ determination and resolution is crucial.

One area of physics $D \emptyset$ has been eager to join is the search for the one remaining piece 

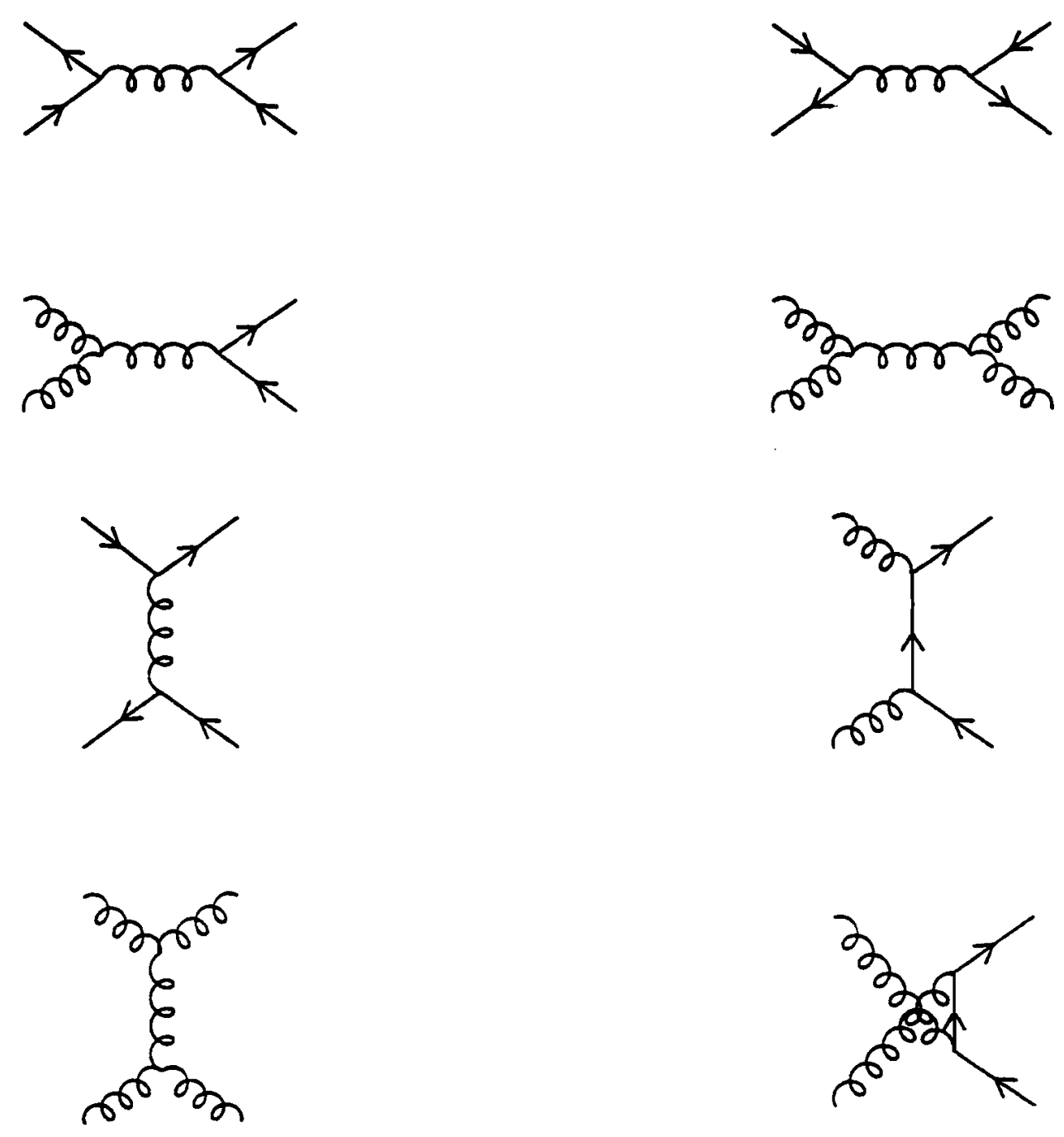

Figure 2.3: The Feynman diagrams for parton-parton scattering in lowest order. Quarks are represented by the straight lines, gluons by the spiral lines. 

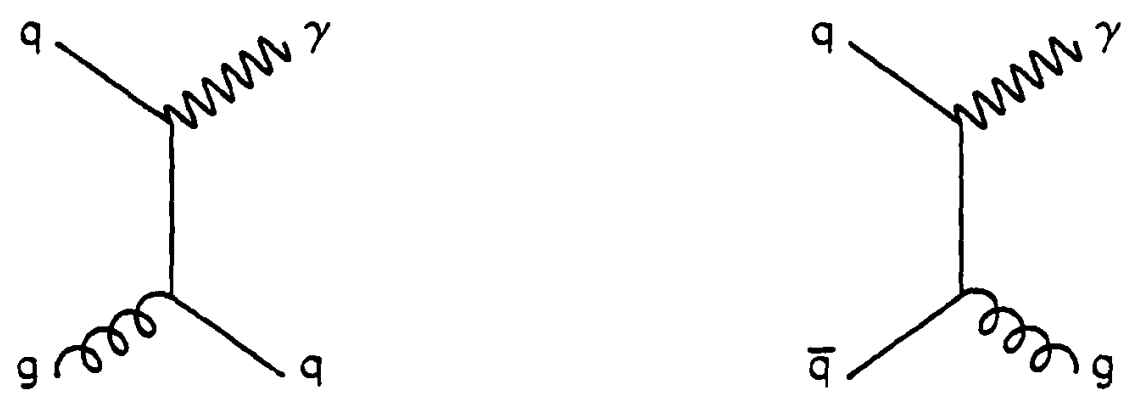

Figure 2.4: The two dominant Feynman diagrams contributing in lowest order to the production of direct photons. The $q g \rightarrow \gamma q$ process will dominate the $q \bar{q} \rightarrow \gamma g$ process.
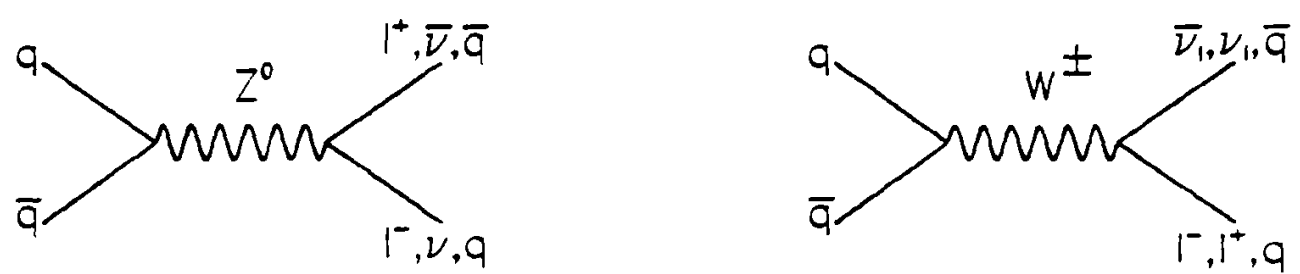

Figure 2.5: The lowest order Feynman diagrams for $Z^{0}$ and $W^{ \pm}$decay. The $Z^{0}$ decays to two fermions, either a lepton (1) and its antiparticle or a quark and its anti-quark. The $W^{ \pm}$will decay to a fermion and the anti-particle of the fermion's doublet partner. 
of the standard model picture, the top quark. The major decay channels for top production are shown in Figure 2.6. Due to their low background, the most promising channels are single lepton + jets $+Z_{T}$ and leptons + jets $+Z_{T}$. In this analysis, all the above detector design goals are necessary. Good lepton identification, good $\nabla_{\mathrm{T}}$ measurement and good jet resolution are all important both to find the top and to measure its mass and width.

An interesting problem with the SM Lagrangian is that there is no mechanism within it to give mass to the fermions. Experimentally, the fermions have been observed to have mass. Yet, the theory does not predict the masses. The solution to this problem is the postulated existence of the Higgs boson, a scalar field which arises from the spontaneous breaking of the $S U(2)$ symmetry. The Higgs boson couples to all massive particles and most strongly to the most massive. Possible decay and discovery channels would be through its coupling to the $\mathrm{Z}^{0}$ and $\mathrm{W}^{ \pm}$, as shown in Figure 2.7. In the $\mathrm{Z}^{0}$ channel, the signal would consist of two leptons or jets adding up to the mass of the $Z^{0}$ boson, plus another two leptons or jets from the $\mathrm{H}^{0}$. The $\mathrm{W}^{ \pm}$channel signal would consist of one or more leptons plus $Z_{\mathrm{T}}$ plus one or more jets.

In all of these analysis, good lepton identification, good energy measurement, good energy resolution, and minimization of $\mathbb{F}_{\mathrm{T}}$ is essential. Chapter 3 will discuss the design characteristics of the $D \emptyset$ detector which were optimized for doing a wide range of physics analyses, in particular the addition of the Intercryostat Detector, and Chapter 7 will briefiy look at the impact the Intercryostat Detector has on the performance of the $D \emptyset$ detector. 

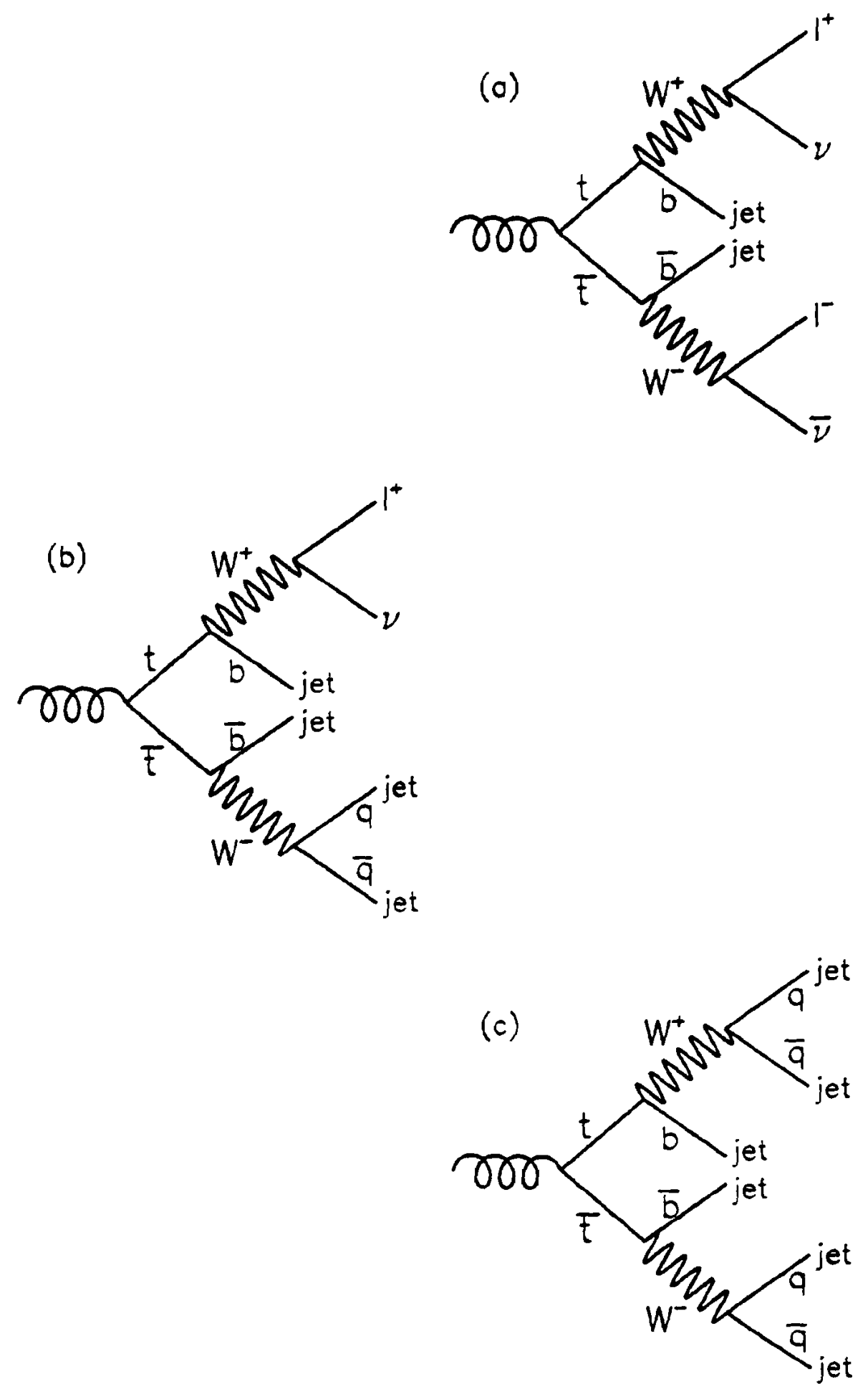

Figure 2.6: The lowest order decay channels for top production. In (a), both $W^{ \pm}$decay leptonically giving a signal of two leptons, jets and $E_{T} ;$ in (b), one $W^{ \pm}$decays leptonically and one $W^{ \pm}$ hadronically giving a signal of one lepton, jets and $E \mathrm{~T}$; in (c) both $W^{ \pm}$decay hadronically. 
I

1

1

(a)

1

1

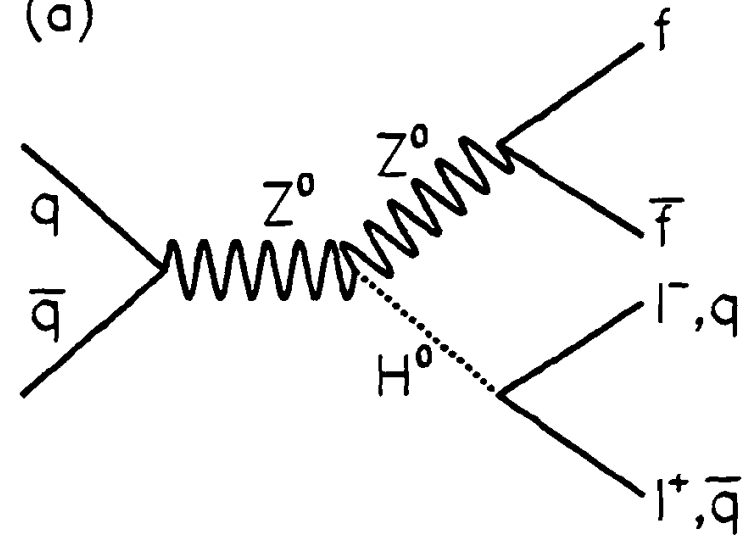

(b)

1
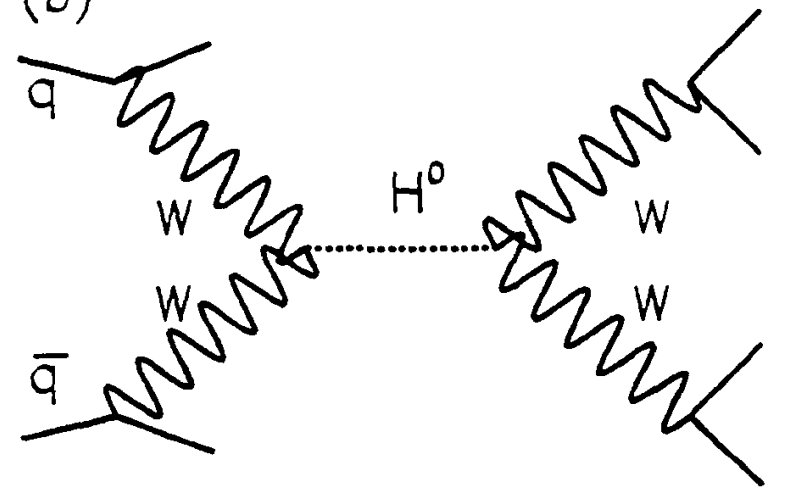

Figure 2.7: Lowest order channels for possible discovery of the Higgs by its decay via the $\mathrm{Z}^{0}$ and $\mathrm{W}^{ \pm}$. 


\section{CHAPTER 3}

\section{APPARATUS}

The $D \emptyset$ experiment was originally proposed in 1983 . It was planned for the D0 interaction region at the Fermi National Accelerator Lab's Tevatron Collider. Installation was completed in early 1992 and the detector started taking data on May 12, 1992. An extensive test beam program was conducted in late 1991 to study the calorimeter, including the ICD. This thesis will primarily concern data taken during that final Test Beam run. In addition, some initial performance results in data taking runs will also be discussed.

The $\mathrm{D} \emptyset$ detector $[13,14]$ is a general purpose detector. Its strengths are its excellent calorimetric energy and spatial resolution, good electron and muon identification, and its high degree of hermeticity (the detector is highly hermetic, i.e., it covers nearly $4 \pi$ of the solid angle). The detector has three major components: the central detector system, used for tracking and electron identification; the calorimetry, which includes a Central Calorimeter, two End Calorimeters, the Massless Gaps, and the Intercryostat Detector; and the muon system, which includes both the large angle and small angle muon counters. In addition there is a scintillator detector, Level 0 , mounted on each End Calorimeter which is used for fast triggering and for luminosity monitoring.

\subsection{The Tevatron Collider}

The $D \emptyset$ detector is currently running at the highest energy particle accelerator in the world at $1.8 \mathrm{TeV}$. The basic principle behind a particle accelerator is simple: a charged particle is given an energy boost as it crosses a gap which has an electromagnetic field across it. Many such gaps can be crossed by lining them up in a 'linear' accelerator. Alternately, a 
single gap can be reused by containing the particles in a circular orbit which allows them to pass through the gap many times per second, as is done at the Tevatron. The particles are maintained in the orbit by a magnetic field. The strength of this field must increase in a synchronized fashion with the increased energy of the particles-hence the moniker 'synchrotron.' In either case the number of crossings, and thus particle acceleration, is limited mainly by technology, size, and money.

After acceleration in a synchrotron, the accumulated particles can be used in one of two ways: (i) fixed target mode or (ii) collider mode. Each mode is used for specific purposes. In fixed target mode, the accelerated particles are extracted in bunches and steered (with magnets) either directly into detectors or onto various targets to produce a wide range of charged and neutral particles. These new particles can then be delivered to waiting detectors. The major advantage of the fired target mode is the control available to the researcher to change the particle type and energy.

In collider mode, the circulating beams of particles are strongly focussed to a headon collision at an interaction region which is surrounded by a detector which catches the resultant debris. The advantage of the collider mode is that much higher energies are available than in fired target mode. This can be seen from a simple calculation of the center of mass energy, $E_{c m}$. Consider two incident particles with energies

$$
\mathrm{E}_{1}=\sqrt{\vec{p}_{1}^{2}+m_{1}^{2}} \text { and } \mathrm{E}_{2}=\sqrt{{\overrightarrow{p_{2}}}^{2}+m_{2}^{2}}
$$

where $\vec{p}_{1}$ and $\vec{p}_{2}$ are the 3 -vector momenta. The 4 -vector momenta are $p_{1}$ and $p_{2}$. The energy available in any collision is

$$
\begin{gathered}
E_{c m}=\sqrt{p_{1}+p_{2}} \\
E_{c m}=\left[\left(E_{1}+E_{2}\right)^{2}-\left(\vec{p}_{1}+\vec{p}_{2}\right)^{2}\right]^{1 / 2} \\
E_{c m}=\left[m_{1}^{2}+m_{2}^{2}+2 E_{1} E_{2}\left(1-\beta_{1} \beta_{2} \cos \theta\right)\right]^{1 / 2}
\end{gathered}
$$

where $E_{1}$ and $E_{2}$ are the energies of the incident particles and $\vec{\beta}_{i}=\vec{p}_{i} / E_{i}$ is the particle velocity. In fixed target mode,

$$
\begin{gathered}
E_{1}=\text { beam energy }=\sqrt{m_{b}^{2}+\vec{p}_{b}^{2}} \\
E_{2}=\text { target energy }=m_{t}
\end{gathered}
$$


$1-$

1

\section{Fermi National Accelerator Lab}

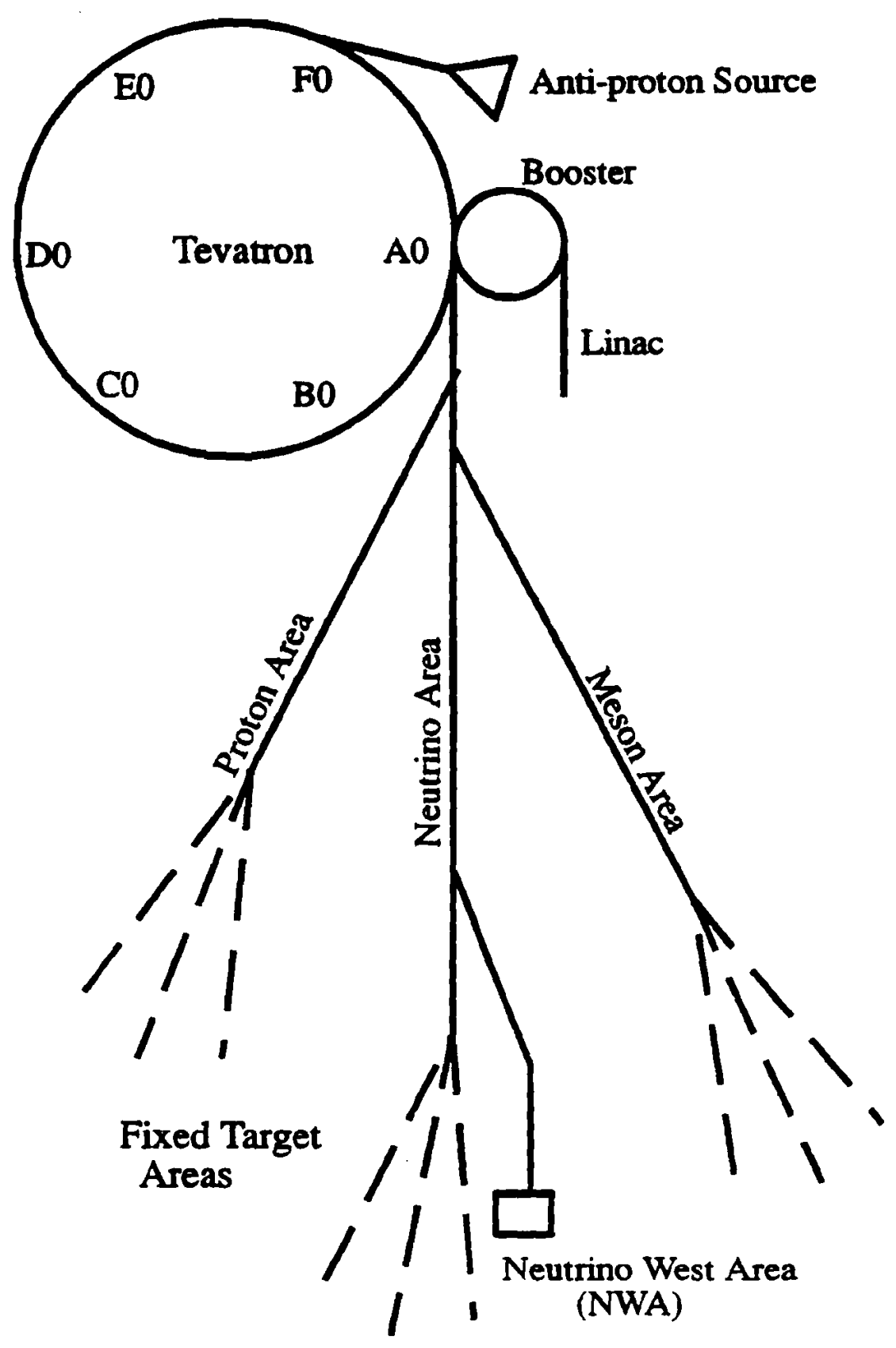

Figure 3.1: The schematic layout of Fermilab's Tevatron collider and the fired target areas. The $D \emptyset$ detector is locate at the $D O$ interaction region in the Tevatron and test beam took place at the Neutrino West Area (NWA) of the Fixed Target area. 
and the velocity of the target is zero. Thus the center of mass energy in fixed target mode is

$$
E_{c m} \equiv \sqrt{s}=\left[m_{b}^{2}+m_{t}^{2}+2 E_{b} m_{t}\right]^{1 / 2}
$$

In current accelerators, the mass of the incident particle and of the target are typically much smaller than the beam energy. With that approximation, the center of mass energy becomes

$$
\sqrt{s}=\sqrt{2 \mathrm{E}_{b} m_{t}}
$$

In collider mode, with two beams of identical particles with equal energy,

$$
E_{1}=E_{2}=\sqrt{m^{2}+p^{2}}=E_{b}
$$

and

$$
\sqrt{s}=\left[2 m^{2}+2 E^{2}\left(1-\beta^{2}\right)\right]^{1 / 2} \text {. }
$$

Substituting for $\beta^{2}=\left|\vec{p}^{2}\right| / E^{2}$ and with a little math, one finds

$$
\sqrt{s}=2 E_{b}
$$

At the Tevatron, each particle beam (proton and anti-proton) is a $900 \mathrm{GeV}$ beam, giving a center of mass energy of $\sqrt{s}=1.8 \mathrm{TeV}$. If instead, a $900 \mathrm{GeV} \bar{p}$ beam were incident on a fuxed $p$ target, the center of mass energy would be only $42 \mathrm{GeV}$. Thus the energy available for study in fixed target mode is much lower than in the colliding mode.

Fermi National Accelerator Lab (FNAL or Fermilab) is in Batavia, IL, about 40 miles west of Chicago. Ferrnilab's current collider machine is known as the Tevatron [15?. It is a $p \bar{p}$ collider with a center of mass energy of $\sqrt{s}=1.8 \mathrm{TeV}$. The current luminosity is $L=\sim 5 \times 10^{30} \mathrm{~cm}^{-2} \mathrm{~s}^{-1}$. The high energy attainable at the Tevatron is available through the use of superconducting magnets. The general layout of the Tevatron is shown in Figure 3.1. Protons, which have been accelerated in the linear accelerator ('linac'), are injected into the booster where their energy is increased to $8 \mathrm{GeV}$. From the booster, they are injected into the Main Ring, where they are accelerated to $120 \mathrm{GeV}$ before being injected into the Tevatron at AO. Antiprotons ( $\bar{p}$ 's) are produced by extracting the $120 \mathrm{GeV}$ 's from the Main Ring at F0 and shooting them at a tungsten target. The resulting negatively charged particles are collected in the debuncher where they circulate until only the $\bar{p}$ 's survive. These $\bar{p}$ 's are transferred to the accumulator and stored as subsequent batches are created. Approximately $10^{7} \bar{p}$ 's can be produced from each batch of $1.8 \times 10^{12} p$ 's. The $p$ 's and $\bar{p}$ 's 
are then accelerated in the Tevatron to approximately $900 \mathrm{GeV}$. There are four interaction regions available: $\mathrm{BO}, \mathrm{CO}, \mathrm{DO}$, and $\mathrm{EO}$, two of which are currently in use. $\mathrm{BO}$ is home to CDF (Collider Detector Facility) and DO is home to the D $\emptyset$ Detector. The fixed target areas are also indicated in Figure 3.1, including the Neutrino West Area (NWA) where the final $D \emptyset$ test beam run took place.

One other point should be noted about the collider construction. The Main Ring and the Tevatron share the same tunnel. At DO, the Main Ring and Tevatron beam pipe separation is increased to several meters. However, the Main Ring roust still pass through the $D \emptyset$ calorimeters.

\subsection{The DØ Detector}

The design of the $D \emptyset$ detector centers on several important features:

- Good calorimetric energy and spatial resolution.

- Good electron identification in the central region.

- Maximum possible muon coverage and muon identification.

- Good missing transverse energy $\left(Z_{T}\right)$ measurement.

- Fine $\eta$ and $\phi$ segmentation ( $\phi$ is the azimuthal angle and $\eta=-\ln (\tan \theta / 2), \theta$ the polar angle).

- Good electron - hadron compensation.

Jets contain both electromagnetic and hadronic energy. However, within the jet, it is impossible to discern how much of the jet energy is of one type or the other. It is therefore important to have the detector response to electromagnetic energy and hadronic energy as similar as possible. The ratio $e / h$ (or $e / \pi$ ) measures the ratio of the detector response to each type of energy and ideally should be as close to one as possible. All of the above goals resulted in the choice of a uranium/liquid argon calorimeter with no central magnetic field, with full muon coverage and central tracking. 


\subsubsection{The Central Tracking Detectors}

As particles move radially outward from the interaction point, the first detector encountered is the Central Detector system $[16,17]$. This system consists of four subsystems. Three of them are concentrically nested devices: the vertex drift chambers (VTX); the transition radiation detector (TRD); and the central drift chambers (CDC). The fourth subsystem consists of two forward/backward drift chambers (FDC) which cap the previous chambers at either end. These detectors start just outside the beryllium beam pipe at a radius of $3.7 \mathrm{~cm}$ and extend to a radius of $78 \mathrm{~cm}$. They cover the range (parallel to the beam pipe) $|z| \leq 135 \mathrm{~cm}$. A cutaway view of the central detectors can be seen in Figure 3.2.

\section{The Vertex Drift Chambers}

The innermost detector is the vertex chamber [16]. Its purpose is to provide a precise position determination of any secondary vertices and to provide a veto to reject any photons which may convert before the TRD. The VTX chamber consists of three layers of cylindrical drift chamber cells, concentric and parallel to the beam pipe. It uses a gas mixture of $95 \% \mathrm{CO}_{2}$ and $5 \%$ ethane at atmospheric pressure. Each cell contains 8 sense wires. The cylinders are carbon, layered with Kapton and aluminum strips which are used to shape the field. The spatial resolution of the VTX is $50 \mu m$ over most of the cell. In addition, hits on two tracks within a cell are found with $90 \%$ efficiency when the separation of the tracks is greater than $700 \mu \mathrm{m}$.

\section{The Transition Radiation Detector}

The TRD $[18,19]$ makes up the next three concentric layers of detection beyond the VTX. The TRD takes advantage of the phenomenon in which $\mathrm{X}$-rays are produced by highly relativistic particles when crossing the boundary (transition) between media with two different dielectric constants. It consists of three cylindrical radiator/detector units. The radiator consists of $18 \mu \mathrm{m}$ polypropylene foils separated by an average gap of $150 \mu \mathrm{m}$, formed by pressing the foils with an indented pattern. The radiator stack is followed by a $15 \mathrm{~mm}$ gap, filled with a mixture of $90 \% \mathrm{Xe}$ and $10 \% \mathrm{C}_{2} \mathrm{H}_{6}$, for ionization cluster formation and photon detection. The clusters drift radially outward towards anode wires at the center of cells formed by the potential and grid wires. Each detector unit is divided into 256 cells 


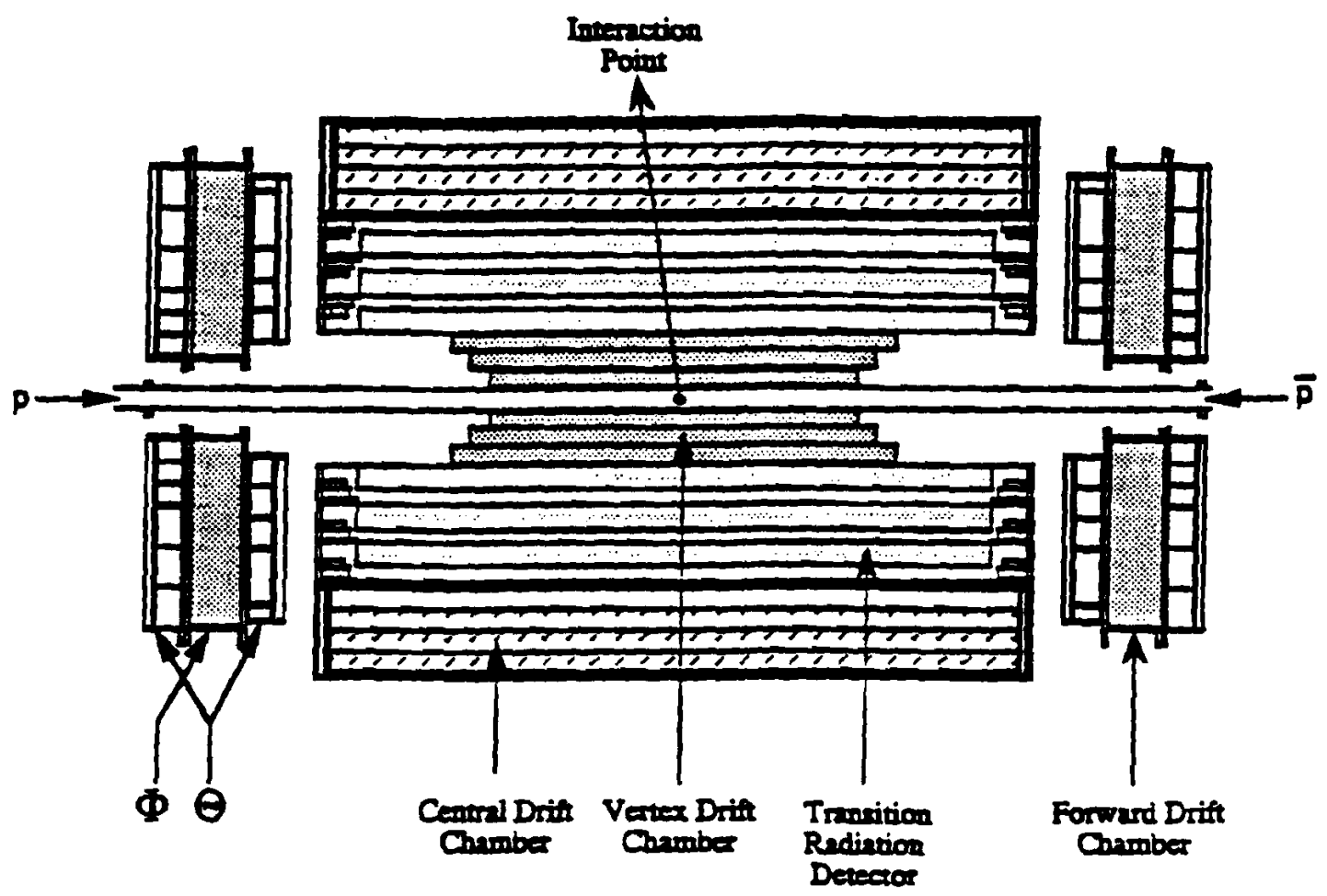

Figure 3.2: A cutaway view of the Central Detector systems. Moving radially outward, the detectors are: the Verter Drift Chamber, the Transition Radiation Detector and the Central Drift Chamber. The Forward Drift Chambers cap the CD system on either side. 
aximmthally. The TRD has been found to have a 50:1 rejection factor for electrons versus picns, for an electron efficiency of $90 \%[20]$.

\section{The Central Drift Chamber}

Beyond the TRD are the four cylindrical, concentric layers of the CDC [16, 21]. Each layer is divided into 32 cells in the $(r, \phi)$ plane. Each cell contains 7 sense wires and 2 delay lines parallel to the beam direction. The sense wires are split between the inner and outer walls of each layer. Five of the sense wires are located along the inner radius, two along the outer radius. Thus each particle traversing this chamber will be seen by 28 sense wires and 8 delay lines. The delay lines are embedded in the outer and inner walls of each cell for measurement of the longitudinal coordinate. The chamber uses a gas mixture of $93 \% \mathrm{Ar}$, $4 \% \mathrm{CH}_{4}$, and $3 \% \mathrm{CO}_{2}$ at atmospheric pressure. The sense wires are staggered by $\pm 200 \mu \mathrm{m}$ to resolve the left-right ambiguity and adjacent layers are shifted by a half cell.

The CDC was tested with both cosmic rays and in the 1990 test beam run. The drift velocity was found to be $37 \mu \mathrm{m} / \mathrm{ns}$ with a maximum drift distance of $7 \mathrm{~cm}$. The resolution of the inner and outer sense wires was found to be $230 \mu \mathrm{m}$ and $330 \mu \mathrm{m}$, respectively. The efficiency for seeing two tracks was measured to be $90 \%$ for tracks separated by 65 ns or $2.5 \mathrm{~mm}$. $d E / d x$ measurements yielded a rejection factor for double minimum ionizing particle tracks of $95 \pm 13[22]$.

\section{The Forward Drift Chambers}

The FDC $[16,23]$, which caps either end of the Central Detector system, consists of two types of chamber: a $\phi$ chamber with a radial wire orientation; and a $\theta$ chamber with wire orientation parallel to the $\mathrm{X}$ axis for the top/bottom sub-chambers and parallel to the $\mathrm{Y}$ axis for the left/right sub-chambers. Each FDC unit consists of two $\theta$ chambers with a $\phi$ chamber between them. The $\phi$ chamber has 16 layers of radial sense wires of length $50 \mathrm{~cm}$. The maximum drift distance is $5.3 \mathrm{~cm}$. The chambers use the same gas mixture as the CDC. The $\theta$ chambers have 8 layers of sense wires. The two $\theta$ chambers are staggered at a $45^{\circ}$ with respect to each other. Sense wires in both the $\phi$ and $\theta$ chambers are staggered by $\pm 200 \mu \mathrm{m}$. The FDC position resolution is $200 \mu \mathrm{m}$ and reaches $90 \%$ efficiency for double track rejection at $2 \mathrm{~mm}$ track separation [24]. 


\subsubsection{The Calorimeters}

The main part of the $D \emptyset$ detector is the liquid argon calorimeter with uranium, copper or steel absorber. As stated earijer, the calorimetry was designed to meet certain goals. These goals include:

- Good energy resolution for jets and electrons.

- Fine transverse segmentation.

- Multiple longitudinal segmentation.

- Uniform response.

- Good e/ $\pi$ ratio (compensation).

- Good gain stability.

- Radiation hardness.

- Compact and low cost.

- Hermetic and no gaps.

An overview of the $D \emptyset$ calorimetry can be seen in Figure 3.3. The energy measurement principle used is that of liquid ionization sampling. The basic calorimeter cell is shown in Figure 3.4. Layers of absorber material are inter-spaced with readout pads and gaps filled with liquid argon. As a particle passes through the active medium (in the case of DO, LAr), it leaves a trail of ionization, proportional to the energy lost in the active medium. The ionized atoms drift to the readout pads, creating a current. This charge is accumulated in a capacitor. The resulting voltage as the capacitor is discharged forms the readout signal. As the particle passes through each layer of absorber material, it loses some of its energy. By including enough absorber material in the calorimeter, all of the particle shower can be contained. The total energy can be calculated by knowing how much energy is "sampled" in the active material and how much is lost in the inactive (absorber) material. The sampling fraction, which will be discussed in detail later, is given by:

$$
s f=\frac{E_{\text {active }}}{E_{\text {inactive }} T E_{\text {active }}}
$$




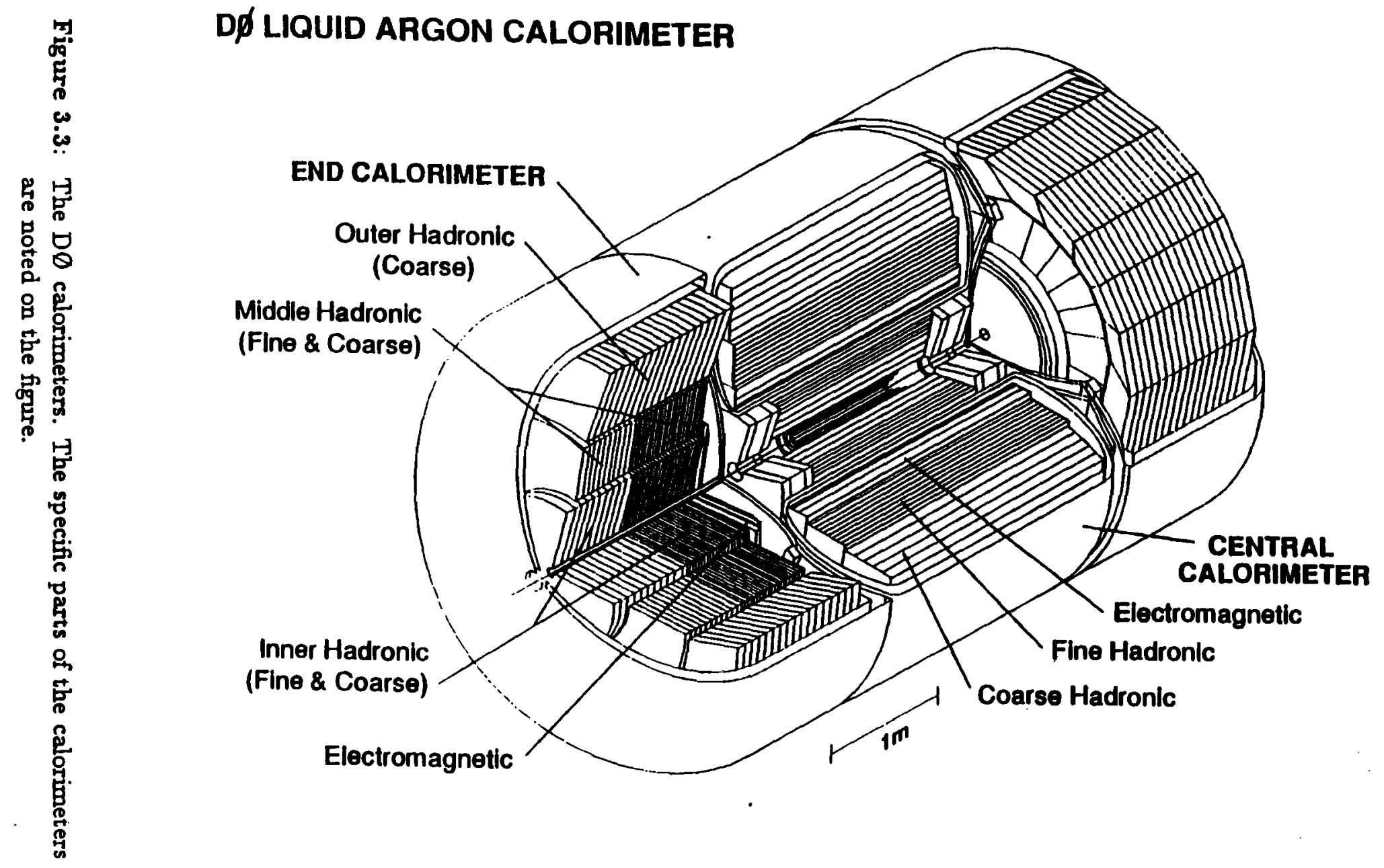




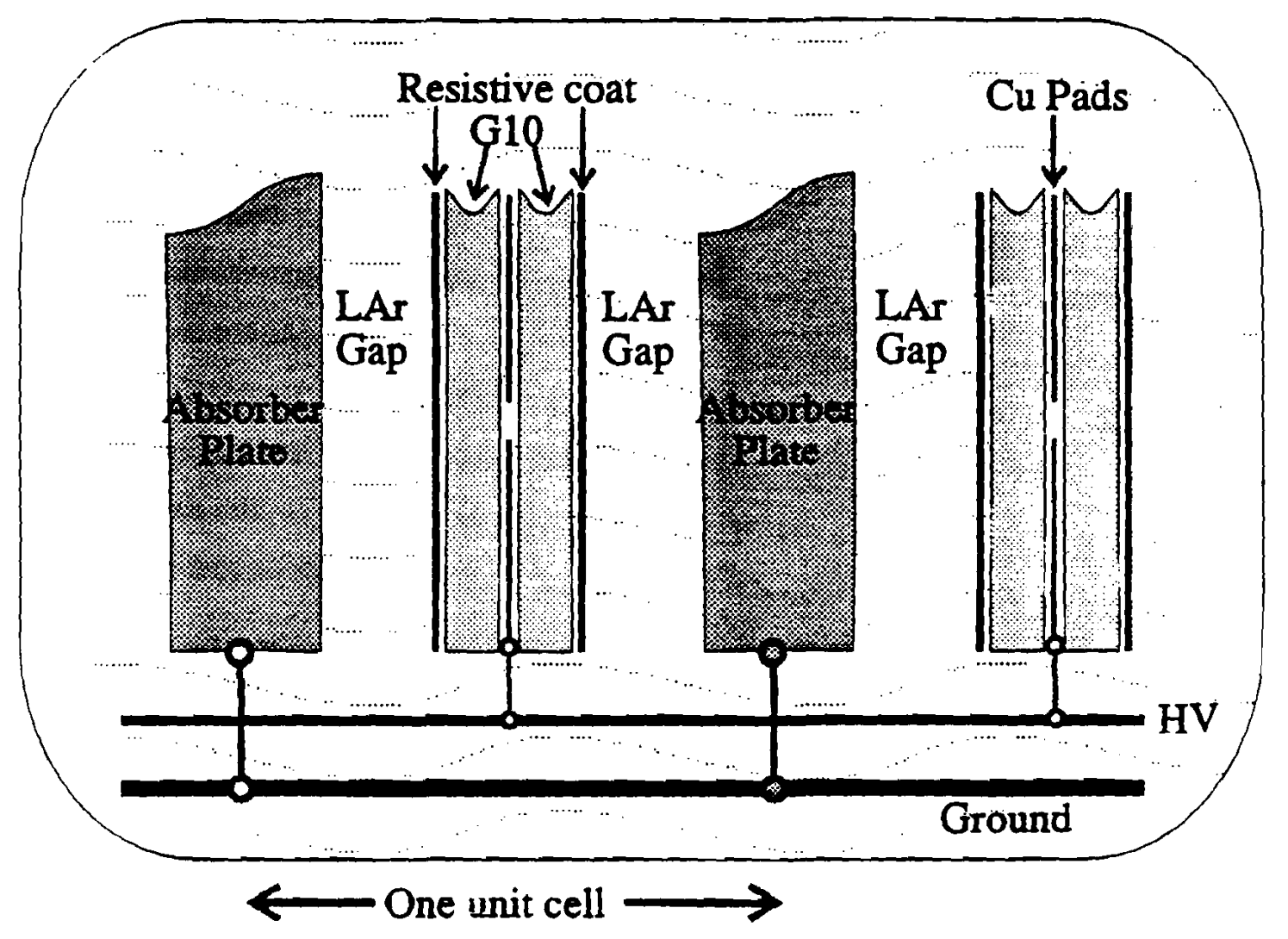

Figure 3.4: A schematic of the basic calorimeter cell. The absorber material in the fine hadronic section is uranium; in the coarse hadronic sections, the absorber is steel or copper. 
The Central Calorimeter (CC) is a cylinder, concentric to the beam axis, with an active region covering the radial range $75<\mathrm{I}<222 \mathrm{~cm}$ from the beam pipe and longitudinal range $226 \mathrm{~cm}$ parallel to the beam pipe. The $\mathrm{CC}$ is made of 64 individual wedge-shaped modules which are arranged in three concentric rings that extend along the entire length of the $226 \mathrm{~cm}$ active region. The three rings are an electromagnetic section, a fine hadronic section and a coarse hadronic section. Each concentric ring is rotated aximuthally by half the arimithal angle to avoid continuous cracks. The CC is contained in a double walled cryostat. Its total weight is over 300 tons.

The CC electromagnetic (CCEM) section is 21 radiation lengths $\left(\mathrm{X}_{0}\right)$ or 0.76 nuclear interaction lengths $\left(\lambda_{0}\right)$ in depth. Its coverage range is $35^{\circ} \leq \theta \leq 145^{\circ}$ and it has 32 modules. Its absorber plates are $3 \mathrm{~mm}$ thick depleted uranium with a $2.3 \mathrm{~mm}$ argon gap for a sampling fraction of $\sim 12.9 \%$. Each CCEM module has 21 radial cells (i.e., 21 cells consisting of an absorber plate and liquid argon gaps) which are arranged in four readout layers. The readout layers are respectively $2,2,7$, and $10 \mathrm{X}_{0}$ deep and are denoted CCEM1, CCEM2, CCEM3 and CCEM4. The third readout layer, which is at shower maximum, has a finer segmentation of $\Delta \eta=\Delta \phi=0.05$ to improve position resolution.

The fine hadronic (CCFH) section extends approximately $3.24 \lambda_{0}$ in depth. Its absorber plates are $6 \mathrm{~mm}$ uranium-niobium (1.7\%) alloy plates with a $2.3 \mathrm{~mm}$ argon gap for a sampling fraction of $\sim 6.9 \%$. There are 16 modules with 50 radial cells each. The modules are divided into three readout layers of $1.3,1.0$ and $0.9 \lambda_{0}$, which are denoted CCFH1, CCFH2 and CCFH3.

The coarse hadronic ( $\mathrm{CCCH}$ ) or leakage section also has 16 modules and is a single readout layer $3.2 \lambda_{0}$ thick. It uses $46.5 \mathrm{~mm}$ copper plates, with a $2.3 \mathrm{~mm}$ argon gap for a sampling fraction of $1.7 \%$. The total CC depth is $6.93 \lambda_{0}$.

The CCEM energy resolution was measured in a FNAL test beam [25] with electrons of energies $10 \mathrm{GeV} / \mathrm{c}$ to $150 \mathrm{GeV} / \mathrm{c}$. The resolution was found to be $\sigma / \sqrt{E}=16 \%$. The position resolution for CCEM3 (at shower maximum) was found to be $2 \mathrm{~mm}$.

\section{The End Calorimeters}

The two End Calorimeters (EC) are divided into four sections: the forward electromagnetic (EM), the inner hadronic (IH), the middle hadronic ring (MH), and the outer hadronic ring $(\mathrm{OH})$. Each EC is contained within its own double set of cryostat walls. 
The ECEM consists of two circular modules covering the forward and backward regions in the range $3^{\circ}<\theta<27^{\circ}$. These modules are approximately 21 radiation lengths in depth and have 21 radial cells. The absorber material is $3 \mathrm{~mm}$ depleted uranium. The signals are ganged in four readout layers of $2,2,7$ and 10 cells each, corresponding to depths of 2.6, 2.6, 7.8 and $10.4 \mathrm{X}_{0}$, respectively. These layers extend from an inner radius of 3.125 inches to an outer radius varying between $80.3 \mathrm{~cm}$ to $104.4 \mathrm{~cm}$. The transverse segmentation matches the standard $\Delta \phi=\Delta \eta=0.1$ calorimeter segmentation throughout most of the ECEM. However, above $\eta=3.2$, pad size becomes too small so the segmentation is increased to $\Delta \phi=\Delta \eta=0.2$. As in the CCEM, the third ECEM layer is more finely segmented to improve position resolution at the shower maximum. The segmentation is $\Delta \eta=\Delta \phi=0.05$ for $\eta<2.7 ; 0.10$ for $2.7<\eta<3.2$; and 0.2 for $\eta>3.2$.

Each IH module is a single circular plug which sits directly behind the ECEM. It extends from an inner radius of $8.0 \mathrm{~cm}$ to an outer radius of $86.4 \mathrm{~cm}$. Longitudinally, the $\mathrm{IH}$ is divided into a fine hadronic (IFH) section, and a coarse hadronic (ICH) section. The IFH uses $6 \mathrm{~mm}$ depleted uranium plates in 65 radial cells which are arranged in four readout layers. The ICH uses $41.3 \mathrm{~mm}$ steel plates in 16 cells and is a single readout layer. The total IH depth is 9.3 interaction lengths. Transversely, the IH matches the ECEM segmentation. For $\eta<3.2$, the segmentation is $\Delta \eta=\Delta \phi=0.1$. For $\eta>3.2$, the IH pad size increases to $\Delta \eta=\Delta \phi=0.2$, and above $\eta=3.8$ (beyond ECEM coverage), the pad size is increased to $\Delta \eta=0.4, \Delta \phi=0.2$.

The MH ring consists of 16 wedge-shaped modules surrounding the $\mathbb{I H}$. The ring extends from an inner radius of 35.13 inches to an outer radius of 62.0 inches. Each module subtends an angle of $22.5^{\circ}$ and is divided longitudinally into a fine hadronic and coarse hadronic section (MFH and $\mathrm{MCH}$ ) as in the $\mathrm{IH}$. The $\mathrm{MFH}$ consists of 60 radial cells arranged in four readout layers, denoted ECMFH1-ECMFH4. The first cell uses the front plate of the module as its absorber plate; all other cells use $6 \mathrm{~mm}$ U-Nb alloy plates. The transverse segmentation follows the usual pattern. The ECMCH is a single readout layer of 14 cells, which use $46.5 \mathrm{~mm}$ steel absorber plates. The total ECMH depth is about 8.1 interaction lengths.

The $O H$ ring also consists of 16 modules, all of the coarse hadronic (OCH) type. The modules have an inner radius of 63.49 in and an outer radius of 89.00 in. Each module forms a parallelogram with the inner face at an angle of $27.4^{\circ}$ with respect to the $x$-y plane. There are 25 radial cells, read out in three layers, ECOCH1, ECOCH2, and ECOCH3. Each 
ceil uses $46.5 \mathrm{~mm}$ steel absorber plates except for the first and last cells. These cells use stainless steel plates which are also used to define the structure of the $O H$.

\section{The Massless Gaps}

In the crossover region from $\mathrm{CC}$ to $\mathrm{EC}$, there are several $\eta$ regions where a particle must travel through mostly support structures (e.g., cryostat walls, end support plates, etc.) be-

fore (or after) reaching the sampling calorimeter modules (this will be discussed in more detail later). To partially compensate for the energy loss in these support walls, an additional layer of LAr sampling was included on the face of each ECMH and ECOH module and on each end of the CCFH modules. These 'massless gaps' (MG) have no significant absorber material but do sample the shower energy before and after the dead material between the cryostats. The CCMG's cover the $\eta$ range, $0.7<\eta<1.2$. The ECMH MG's cover the range, $1.1<\eta<1.4$ while the ECOH MG's cover $0.8<\eta<1.1$. The segmentation matches the usual $\Delta \eta=\Delta \phi=0.1$.

\section{Calorimeter Electronics}

The calorimeter electronics consists of three main parts: the charge sensitive preamps, the shaping and sample-and-hold circuits, and the Analog to Digital converters [26]. There are over 50,000 readout channels in the $\mathrm{D} \emptyset$ calorimetry. Each cryostat has four feedthrough ports in order to bring out the signals. The signals are organized into projective towers in $\eta$ and $\phi$. These towers are then used to provide the Level 1 trigger.

The calorimeter preamps are FET-based hybrid charge sensitive preamps. The feedback capacitance of the preamps is $5 \mathrm{pF}$ in most of the calorimeter. This is changed to $10 \mathrm{pF}$ for CCEM3, where the electromagnetic shower maximum occurs and where the segmentation changes from the nominal $\Delta \eta=\Delta \phi=0.1$ to $\Delta \eta=\Delta \phi=0.05$. The design of the preamps emphasizes a low rms noise of $\sim 2000$ electrons with a noise slope of $\sim 3000$ electrons $/ \mathrm{nF}$. A pulser system is used to monitor the gain differences between the preamps.

Signal shaping and sampling is accomplished at the front end of the base line subtractor (BLS) hybrid. The signal is first shaped with a $250 \mathrm{~ns}$ integration and a $30 \mathrm{~ns}$ differentiation. The double sampling occurs over a $2.2 \mu$ s period, taking advantage of the $3.6 \mu$ s bunch crossing time. The output next passes through a high speed analog trigger pickoff where the signals for all depths are added into the $2 \times 2(\eta \times \phi)$ towers. These projective towers 
are then incorporated into the Level 1 trigger system. The back end of the BLS consists of three analog memory circuits. These are used to hold the baseline sample (taken just before the beam crossing), the peak sample (taken $2.2 \mu \mathrm{s}$ after crossing) and, if a trigger occurs, the difference signal. This difference signal (or output buffer) is later read out by the ADC (analog-to-digital converter) system.

The $A D C$ system is a 12 bit digitizer with a $5 \mu$ s digitization time. Each ADC channel includes pedestal and limit memory. This information can be used to do offset subtraction and suppression of small or zero signal channels.

\subsubsection{The Muon Detectors}

The Muon system [27] plays an integral part in DØ's excellent lepton identification and measurement. The system is broken into two subsystems: the wide angle muon spectrometer (WAMUS) and the small angle muon spectrometer (SAMUS). Each subsystem uses three super-layers of proportional drift tubes (PDT) with magnetized toroids after the first layer of drift tubes. In all, there are five toroids: the central toroid (CF), covering the region between $41^{\circ}$ to $139^{\circ}$; the two end wall toroids (EF), covering the region from $9^{\circ}$ to $43^{\circ}$; and the two SAMUS toroids, covering the region between $2.5^{\circ}$ to $11^{\circ}$. Each toroid is magnetized to a field of $19 \mathrm{kG}$.

\section{WAMUS}

Muon position is determined by the three super-lavers of PDT's. In the WAMUS, each super-layer consists of 3 or 4 planes of PDT's. The first layer, designated layer A, is just inside each of the WAMUS toroids and has four PDT planes. Layer B is just outside each of the toroids and has three PDT planes. Layer $C$, which is situated approximately $135 \mathrm{~cm}$ outside Layer B, also has three PDT planes. The unit cell for each PDT is $10.1 \mathrm{~cm}$ wide by $5.5 \mathrm{~cm}$ high. The top and bottom of each aluminum tube have vernier cathode pads. The central sense wire is a nickel struck gold plated $50 \mu \mathrm{m}$ tungsten wire at $300 \mathrm{~g}$ tension. It is kept at a potential of $+4.54 \mathrm{kV}$ with respect to the grounded $\mathrm{Al}$ cell. The cathodes are copper-clad Glasteel (polyester and epoxy based plastic sheets with a glass fiber mat) which are cut into a repeating diamond pattern. This pattern repeats itself every $61 \mathrm{~cm}$. The cathodes are kept at $+2.60 \mathrm{kV}$. The tubes are filled with a mixture of $90 \%$ argon and $10 \% \mathrm{CO}_{2}[28]$. 
In all, there are 156 wide angle drift chambers and 11032 unit cells. The WAMUS chambers range in size from $254 \mathrm{~cm}$ wide by $579 \mathrm{~cm}$ long by 3 decks high to $142 \mathrm{~cm}$ wide by $335 \mathrm{~cm}$ long by 4 decks high. The minimum momentum necessary for the mon to punch through the toroid is $3.5 \mathrm{GeV}$ at $\eta=0$. The position resolution of the wide angle system is $200 \mu \mathrm{m}$.

Additionally, there is a system of scintillator hodoscopes which sit above the WAMUS system. These hodoscopes provide timing information which is used to veto cosmic rays.

\section{SAMUS}

The small angle muon system consists of six stations of PDT's [29]. The unit cell is a $3 \mathrm{~cm}$ diameter stainless steel tube with a $50 \mu \mathrm{m}$ sense wire through the center. Each station contains three planes of drift tubes. The cylindrical tubes in each plane are arranged in two sub-planes. These overlapping sub-planes are made of rows of offset tubes to compensate for the inefficiency inherent in a circular tube design. The three sub-planes in each SAMUS station are oriented in the three directions, $X, Y$, and $U$, where $U$ is offset by $45^{\circ}$ to resolve multiple tracks. As in the WAMUS system, Stations $A$ and $B$ sit respectively on the inside and outside edge of the SAMUS toroids, while Station C is located $330 \mathrm{~cm}$ outside Station B. The position resolution for the small angle system is about $300 \mu \mathrm{m}[30]$.

\subsubsection{The Trigger System}

\section{The Level 0 Detector}

The Level 0 (LO) Detector $[31,32]$ provides a lowest order trigger for $D \emptyset$. It consists of two arrays of hodoscopes which are mounted on the face of the EC cryostats. The LO scintillation counters surround the beam pipe and provide nearly complete coverage over the range $2.2 \leq|\eta| \leq 3.9$ with partial coverage extending the overall range to $1.9 \leq|\eta| \leq 4.3$. Light guides are used to transport the light to photomultiplier tubes (PMT's) situated on the outside of the array. The scintillator is BC-408, produced by the Bicron Corp. and is 0.625 inches thick. The PMT's are 8-stage Phillips XP228 tubes with a gain of $1 \times 10^{6}$ at $-3000 \mathrm{~V}$.

The hodoscope arrays consist of two different types of counters. Long counters measure 25 inches $\times 2.75$ inches and cover the entire length of the array. The long counters are read 
out by two photomultiplier tubes, one at each end, and have a time-of-flight resolution of $\sim 80$ ps. Short counters, which are 2.75 inches $\times 2.75$ inches, have a single phototube on the outer edge and a time-of-flight resolution of $\sim 120$ ps [33]. Each array is made of two sub-planes, one arranged vertically of short and long counters and the other arranged in the same pattern but horizontally. In Figure 3.5 , the bottom figure shows one of the sub-planes, with the hatched area indicating the scintillator coverage and the clear area indicating the readout wave guides. The top figure shows the crossed sub-planes and the total detector coverage is indicated by the hatched areas.

Level 0 serves several important roles in DO. It provides luminosity monitoring; it can detect multiple interactions within a beam crossing; it can identify actual beam-beam interactions; and it can determine the $z$ coordinate of the interaction vertex. A laser calibration system is used to monitor the performance of the detector [34].

\section{The Level 1 Trigger}

The Level 1 Trigger is a hardware trigger. Its purpose is to provide a fast decision to keep or discard an event by looking at calorimeter energy sums in the event and comparing them to preset conditions. The calorimeter level 1 trigger adds four adjoining cells (covering $\Delta \eta=\Delta \phi=0.2)$ to form a trigger tower. The trigger coverage extends over the range $|\eta| \leq 4.0$, with 2500 separate trigger towers. These trigger towers are summed to produce the variables used for comparison: the global total electromagnetic and hadronic energies, the EM, hadronic and total transverse energies $\left(E_{T}\right)$, and the missing transverse energy (H $\mathrm{T}$ ). The results of the comparisons are used to set a trigger bit which is logically added to the information from the other detector systems (muon, central detectors) to digitize the event and pass it through to the Level 2 system.

\section{The Level 2 Filter}

The Level 2 filter is a software trigger. It consists of 50 Digital Equipment Corp 4000/60 workstations which subject individual events to a more rigorous analysis before a decision is made to $\log$ an event to tape. At this level, there are calculations of jet energies, $\mathbb{F}_{\mathrm{T}}$, comparisons made between the tracking detectors and the calorimeter, muon momentum determination and other event information. These variables must meet a set of preset criteria in order for the event to be written to tape. 


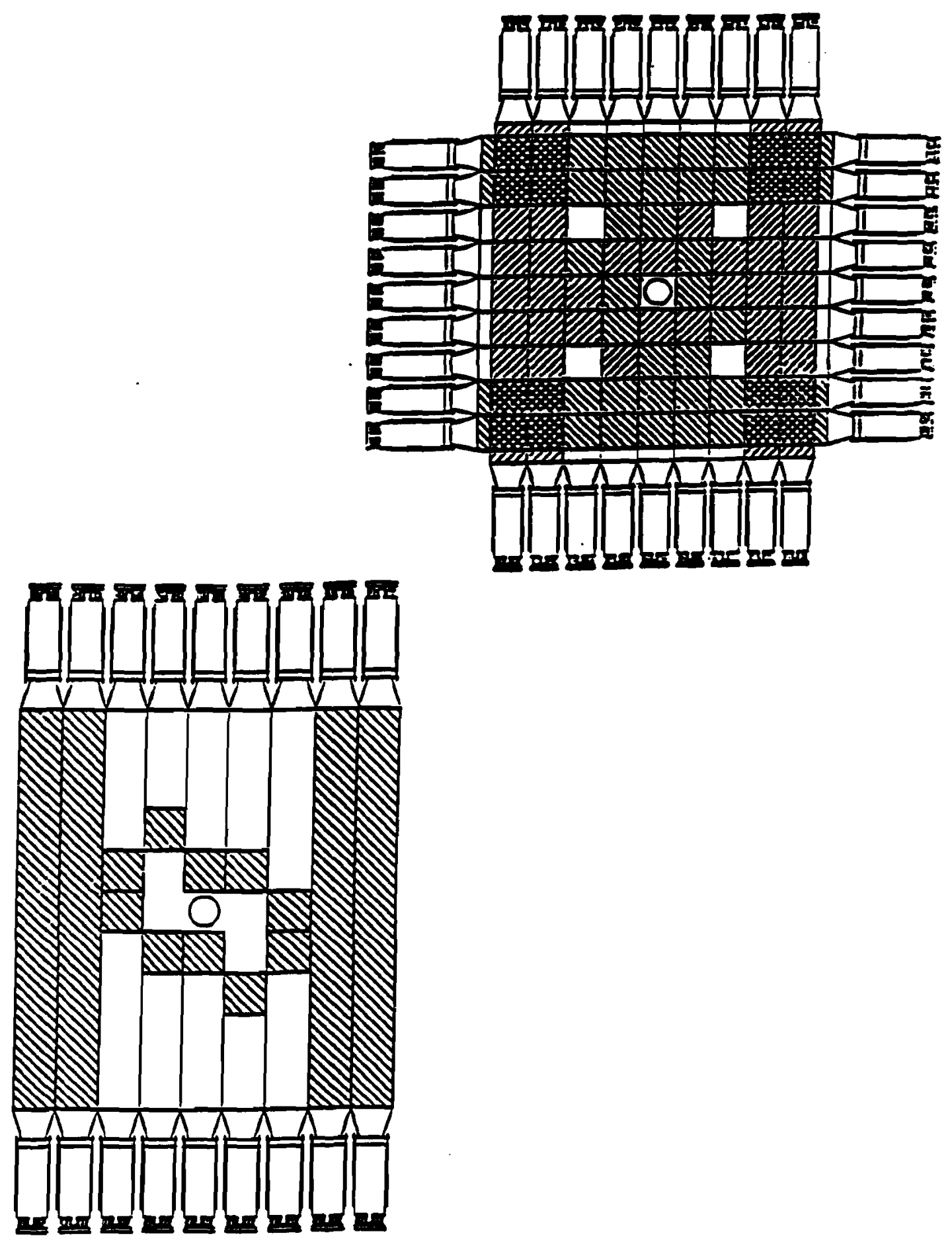

Figure 3.5: A view of the Level 0 Detector. The bottom picture shows one of the sub-planes of the detector. The top picture shows the two crossed sub-planes which make up the detector. Hatched areas represent actual detector (scintillator) coverage. 


\subsection{The Intercryostat Detector}

\subsubsection{Motivation for the Intercryostat Detector}

Early Monte Carlo simulations of the $D \emptyset$ detector $[35,36,37]$ indicated poor energy reconstruction in the region $0.7<\eta<1.4$, where $\eta=-\ln (\tan \theta / 2)$. As shown in Figure 3.6, as much as $40 \%$ of the initial energy escapes detection in this critical $\eta$ region. This energy loss can be easily understood if one considers Figure 3.7, a side view of the $D \emptyset$ calorimeters. Consider a particle travelling along $\eta=1.1$. That particle will pass through very little of the CC calorimetry, perhaps just catching the edge of the CCEM, before going through the do'sble set of CC cryostat walls, through the small air gap between the cryostats and through the double set of EC cryostat walls. Finally, at this point, its energy will be measured by the EC hadronic modules. Of course, by this time, a great deal of the particle's energy has been irrevocably lost in the cryostat walls.

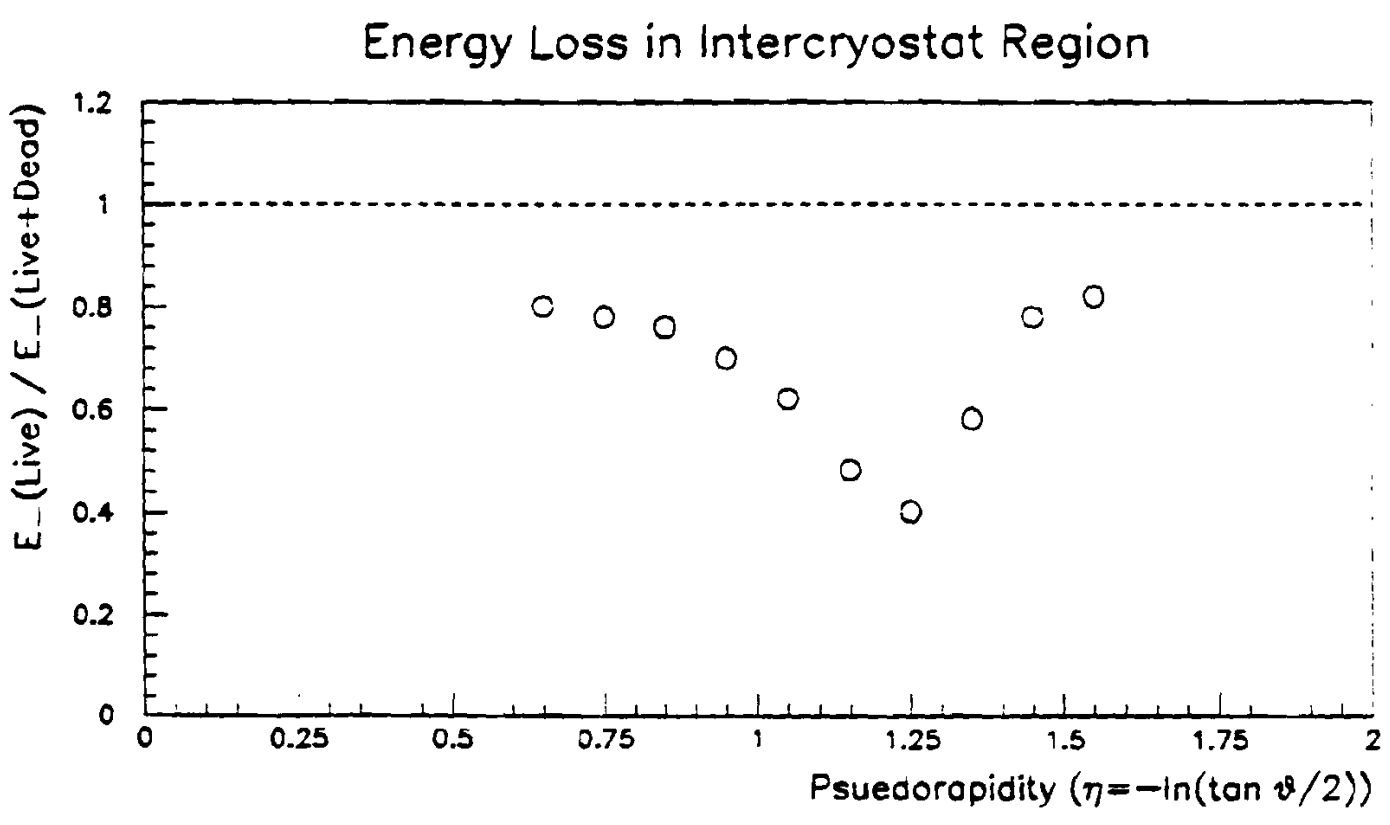

Figure 3.6: Results from an eariy Monte Carlo simulation show the fractional energy lost in the intercryostat region. The live energy over total initial energy is plotted as a function of $\eta$.

Several proposals were introduced by members of the $D \emptyset$ collaboration to soive this 


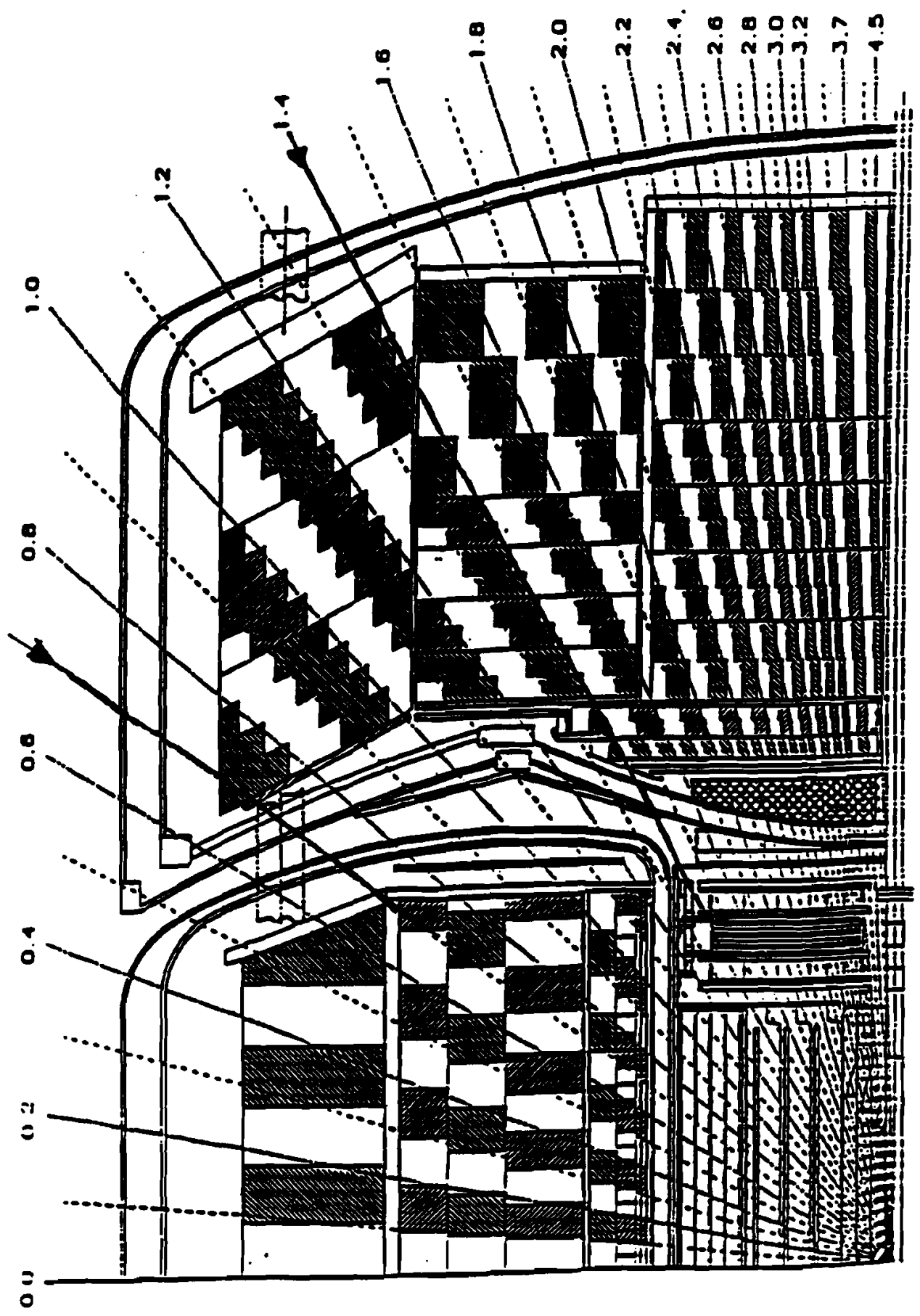

Figure 3.7: Close-up view of the $D \emptyset$ calorimeters. The regions within the indicated $\eta=-\ln (\tan \theta / 2)$ lines have poor overall coverage due to the large amount of dead material (e.g., cryostat walls). 
energy loss problem. One such solution was the massless gaps, as mentioned previously. Another proposed solution was to insert a single layer of scintillator in the small air gap between the CC and EC. As this detector would be situated between the CC and EC cryostats, it became known as the Intercryostat Detector (ICD). The University of Michigan (UM) joined with the University of Florida group in 1988 on an R\&D project to develop the ICD. Subsequently, the UM played a major role in the final design, production, installation and commissioning of the ICD.

\subsubsection{Description of the Intercryostat Detector}

The ICD was subject to several demanding constraints. The D 0 LAr calorimeter is a highly uniform and finely segmented device. It was important that the ICD maintain that segmentation and uniformity. In addition, the space between the cryostats was extremely limited; $\sim 2$ inches was allocated for the ICD. Most importantly, this device had to have a linear response with energy. A scintillator-based detector was able to satisfy all of these criteria. Scintillator is an excellent sampling device, with the light output roughly proportional to the amount of energy deposited in the scintillator. Likewise, a scintillator tile could be made thin enough to fit in the allotted space and easily cut into sizes to maintain the $\Delta \eta=\Delta \phi=0.1$ tower structure of the calorimeter. However, the minimal space available did pose significant difficulties in using the traditional scintillator readout system of light guides. Instead, research focussed on a newer concept: using bundles of wavelength shifting fibers embedded in the scintillator [38] to take the light to a photomultiplier tube. By carefully choosing the distribution of fibers across the surface of the scintillator tiles, a high degree of uniformity can be achieved. The final ICD scintillator tile design is shown in Figure 3.8.

The scintillator tile is made of BC-414 scintillator produced by the Bicron Corporation. BC-414 is a polyvinyltoluene (PVT) based scintillator with a maximum emission at $392 \mathrm{~nm}$. The index of refraction of BC-414 is 1.58. The six tile sizes are given in Table 3.1. Each tile spans 0.1 in $\eta$ and 0.1 in $\phi$. The entire ICD covers the six $\eta$ regions: $0.8 \rightarrow 1.4$ and the 64 azimuthal $(\phi)$ units. Embedded in each tile are either three or four bundles of wavelength shifting fibers (WLS). As light enters the fibers, its wavelength is shifted to a higher wavelength. As the wavelength is shifted, the light rays change direction. This allows the light to be captured through total internal reflection due to the different indices of refraction of 


\section{Schematic of Final ICD Tile Design}

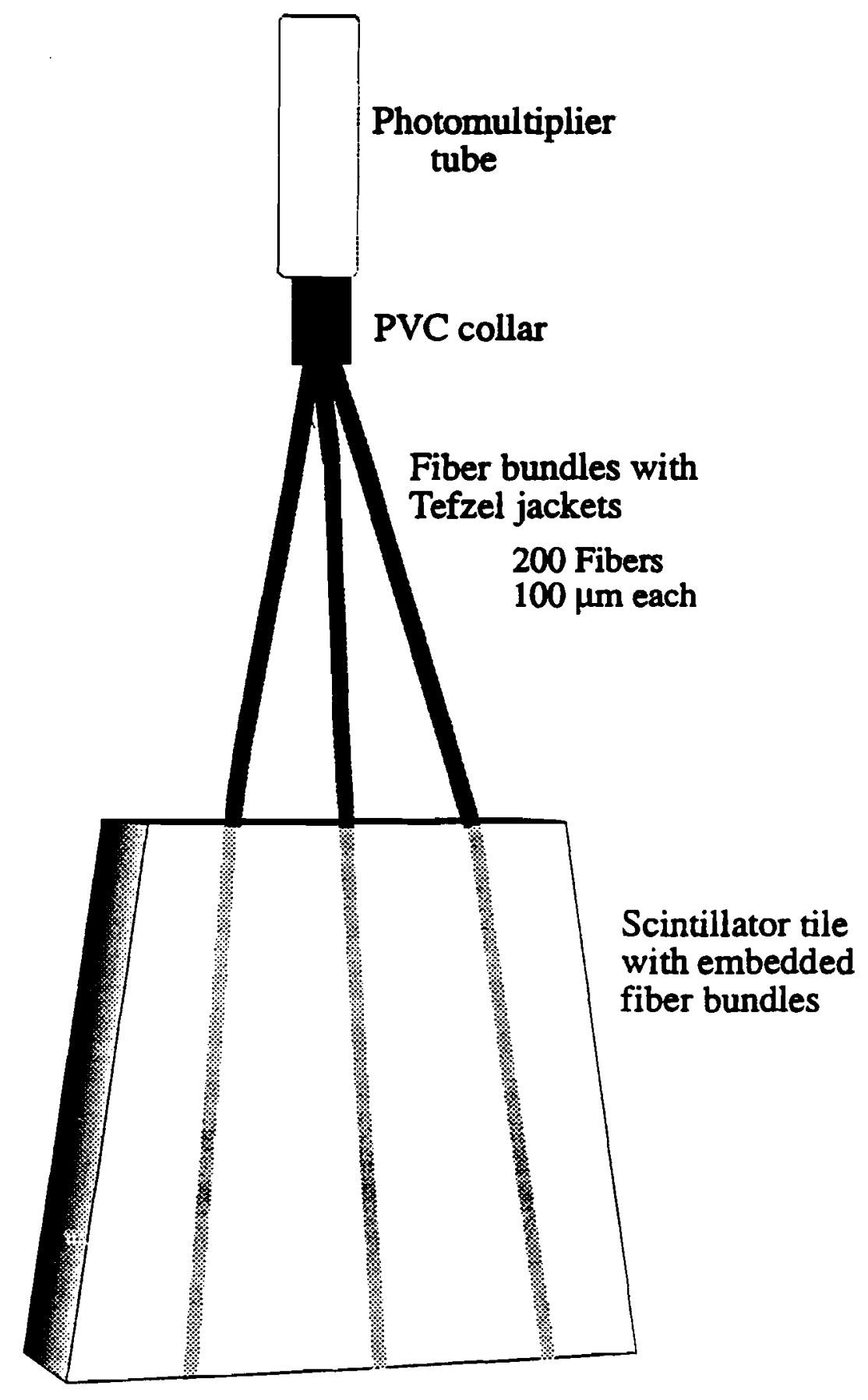

Figure 3.8: The final design for an ICD tile consists of a scintillating tile with embedded fiber bundles. The flexible fiber bundles are gathered into a PVC collar and are viewed by the photomultiplier tube. 
the fiber core and cladding. The fibers are each $100 \mu \mathrm{m}$ in diameter with approximately 200 fibers per bundle. The fibers are made of a polystyrene core with a PMMA (polymethyl methacrylate) cladding. The core and cladding have indices of refraction of 1.60 and 1.49 , respectively. The wavelength shifting dopant is BBOT. The arrangement of fiber bundles embedded in the tile yields a maximum non-uniformity of $10 \%$ across the surface of a tile. The fibers from all bundles in a tile are gathered into a plastic collar and viewed by a 0.5 inches diameter photomultiplier tube (PMT).

\begin{tabular}{|l|c|c|c|c|c|c|}
\hline Tile & 6 & 5 & 4 & 3 & 2 & 1 \\
\hline$\eta$ range (radians) & $1.4-1.3$ & $1.3-1.2$ & $1.2-1.1$ & $1.1-1.0$ & $1.0-0.9$ & $0.9-0.8$ \\
\hline Length (inches) & 5.10 & 6.30 & 7.30 & 5.90 & 6.70 & 7.85 \\
\hline Inner Width (inches) & 2.812 & 3.275 & 3.845 & 4.590 & 5.165 & 5.816 \\
\hline Outer Width (inches) & 3.267 & 3.837 & 4.496 & 5.155 & 5.807 & 6.568 \\
\hline Area (sq. in.) & 15.50 & 22.40 & 30.44 & 28.75 & 36.76 & 48.61 \\
\hline \# of Bundles & 3 & 3 & 3 & 4 & 4 & 4 \\
\hline
\end{tabular}

Table 3.1: Dimensions and specifications for the six sized of ICD tiles.

In all, there are 768 individual tiles (64 $\phi$ units $\times 6 \eta$ units $\times 2$ End Calorimeters). The mechanical housing designed for the ICD stresses modularity and quick replacement, if necessary. There are two modules per $\phi$ unit. Each module consists of an aluminum box covering $3 \eta$ units. Included in each box are the three scintillator tiles, their three PMT's and an electronics card with the three bases and preamps to run the PMT's, as shown in Figure 3.9. The inner module extends from a radius of $\sim 30$ inches to $\sim 50$ inches and covers $1.4>\eta>1.1$. The outer module extends from $\mathrm{I} \simeq 50$ inches to $\mathrm{r} \simeq 70$ inches and covers $1.1>\eta>0.8$. The modules are mounted in two annular rings on the surface of the cryostat, as shown in Figure 3.10. In addition, Figure 3.11 and the frontispiece (at the beginning of this thesis) are photographs taken after installation of the ICD. The two rings of boxes can be seen, with their servicing cables bringing in the HV and calibration light and taking the signals out to the $D \emptyset$ electronics. 


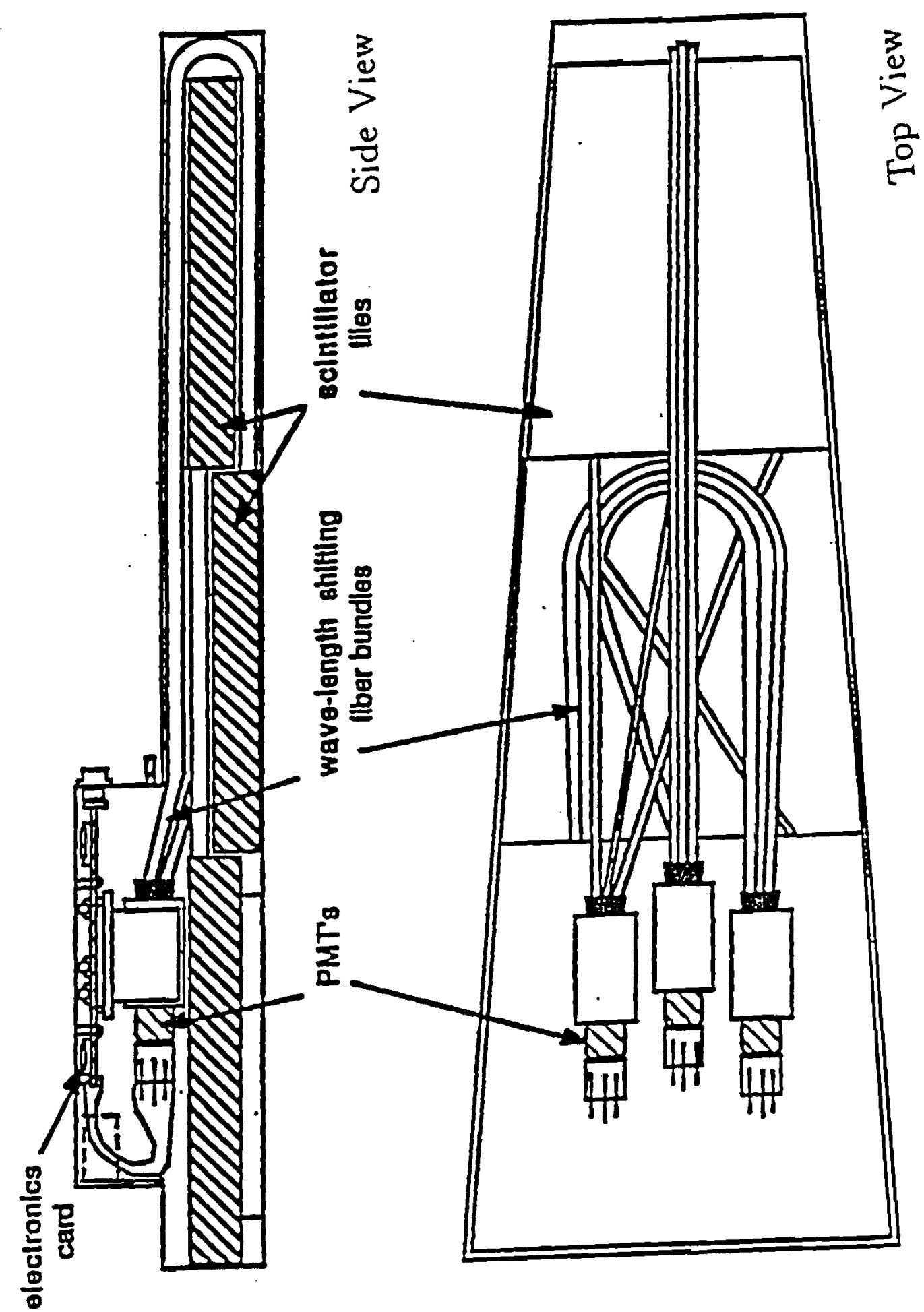

I

Figure 3.9: A complete ICD box. The three tiles are slightly offset vertically within the box to accommodate the fibers bending around to the photomultiplier tubes. The tubes and electronics are contained in the small pod on the top of the box. 


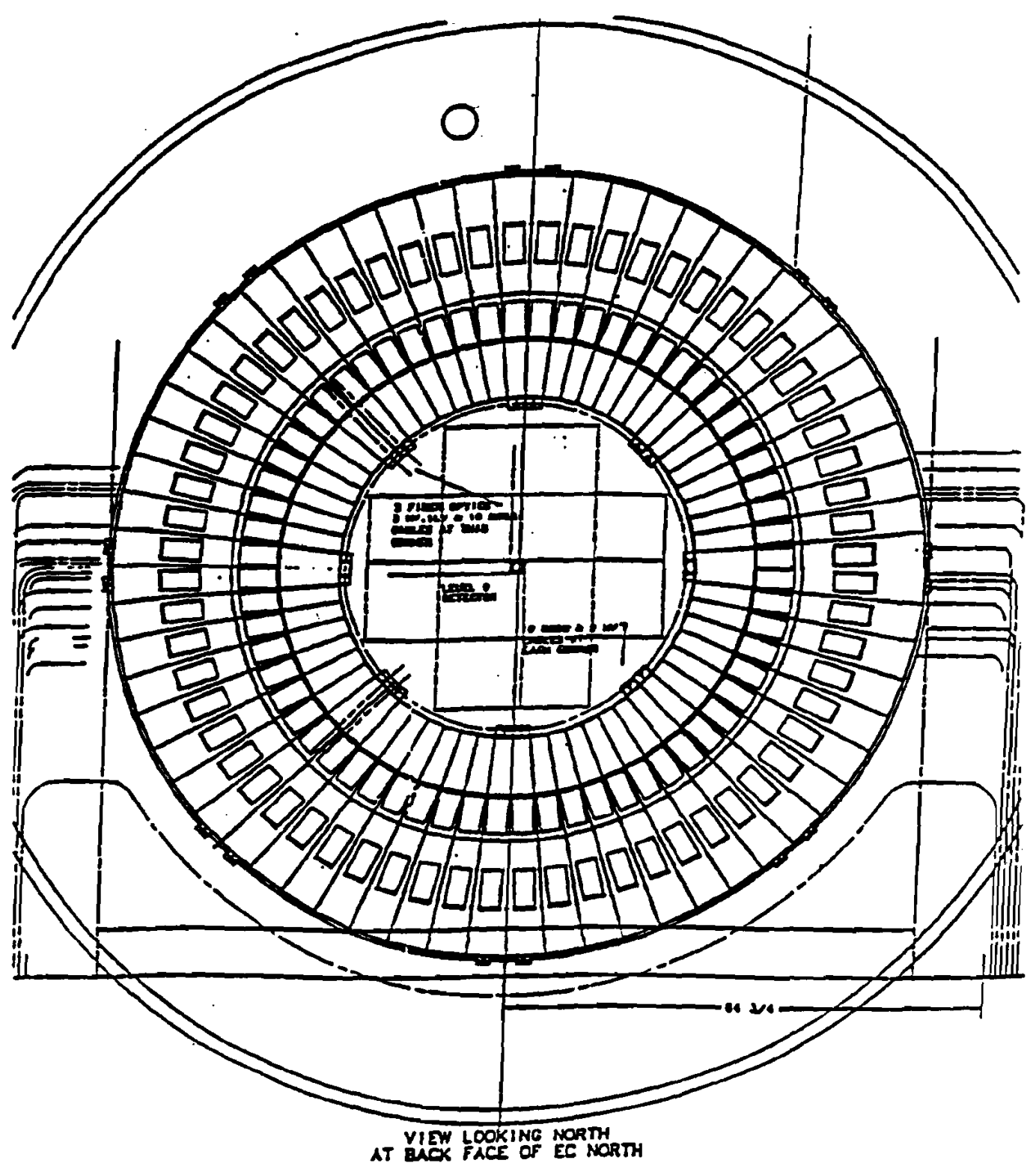

Figure 3.10: End-on view of the two annular rings of ICD boxes mounted on the end cryostats. 


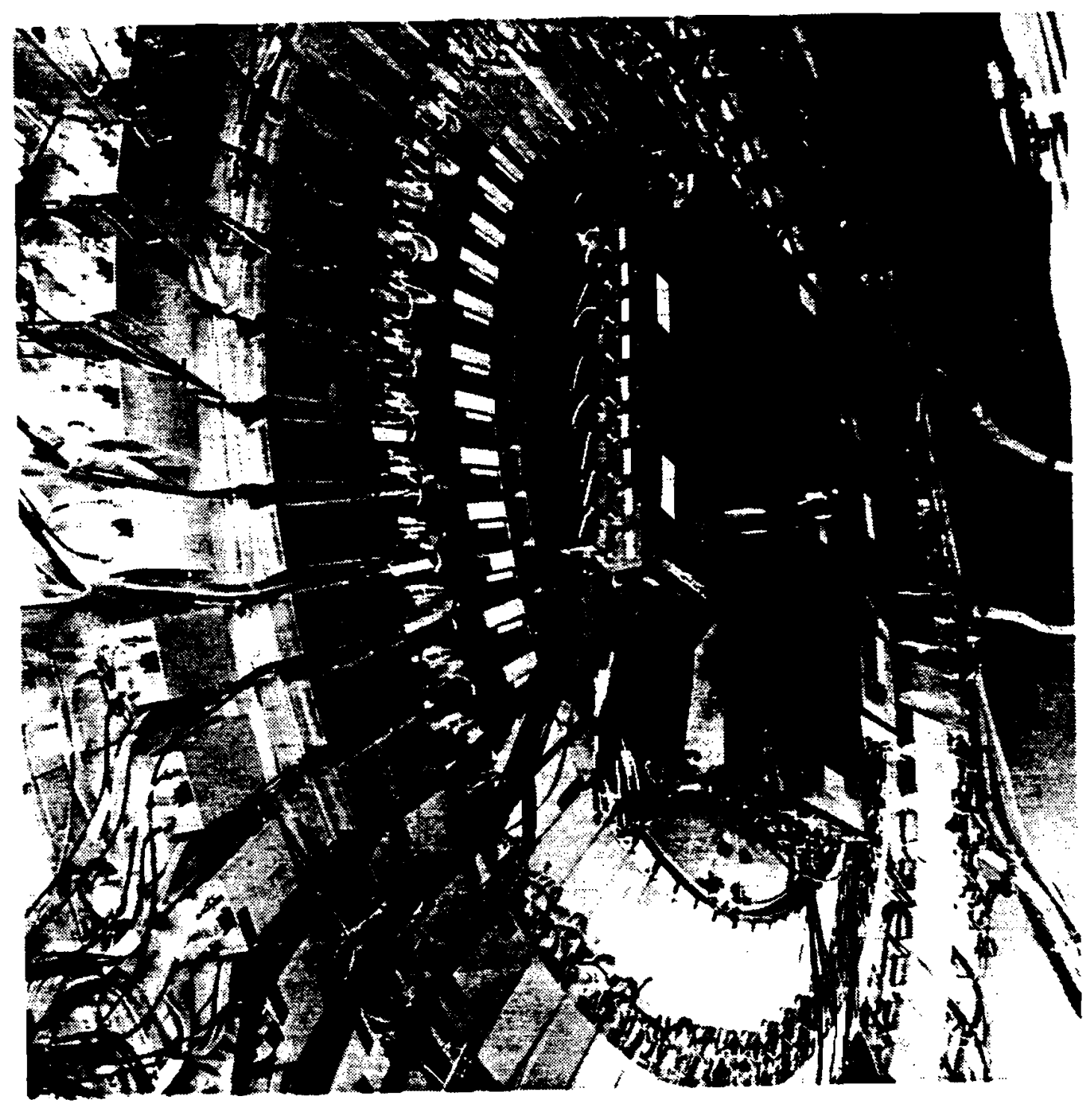

Figure 3.11: A photograph of the installed ICD. This is a close-up view of a section of the two annular rings of ICD boxes mounted on the end cryostats. The service cables for the high voltage and output signais are clearly visible. The Level 0 detector can be seen in the center of the end cryostat, with the beam pipe extending out slightly. 


\subsubsection{Intercryostat Detector Electronics}

The electronics for the ICD consists of several stages. The voltage dividers and charge integrating preamplifiers for the PMT's are located within the ICD boxes. The signals are then taken to specially designed VME boards where they are merged with the calorimeter signals. The signals are then noise subtracted and digitized using the standard DØ BLS (base-line subtractor). The power supplies and digitization electronics are located in a more accessible site, remote from the boxes.

The basic layout for the ICD electronics card can be seen in Figure 3.12. All three tubes in a box are driven from a single high voltage supply. The motivation behind this feature is mainly monetary, as it cuts the high voltage supply and cabling costs considerably. The three tubes in a single box must therefore be well matched for gain-voltage characteristics. The single incoming high voltage feeds directly into one tube and is fanned out to the other two via a resistor chain. The resistors can be used to fine tune any slight mismatching of the PMT's. The design of the voltage dividers emphasizes:

- low gain (factor of about one thousand less than obtained with traditional voltage dividers) in order to increase the lifetime of the tubes in a high intensity calorimeter environment, $G=2 * 10^{4}$;

- low DC current (by a factor of ten) in the voltage divider to protect tube against accidental exposure to large signals (e.g. a beam dump), I = $100 \mu \mathrm{mA}$;

- high efficiency and linearity to exploit the full dynamic range from one minimum ionizing particle to large electromagnetic showers equivalent to 500 minimum ionizing particles;

- low noise to allow precision measurement of pulse heights, $\sigma=1 / 30 \mathrm{MIP}$.

Due to budget constraints, two different types of PMT's were used. All the high 7 tiles and $\sim 25 \%$ of the low $\eta$ tiles are equipped with Hamamatsu R647 phototubes. These are 10 stage tubes with linear focussing dynodes. The remaining tiles use Russian-made PM60 phototubes. These are also 10 stage tubes, but with a different dynode structure in the last stage. Two different voltage divider chains were designed to take into account the differences in the two types of PMT's. The tubes have a nominal operating voltage of 

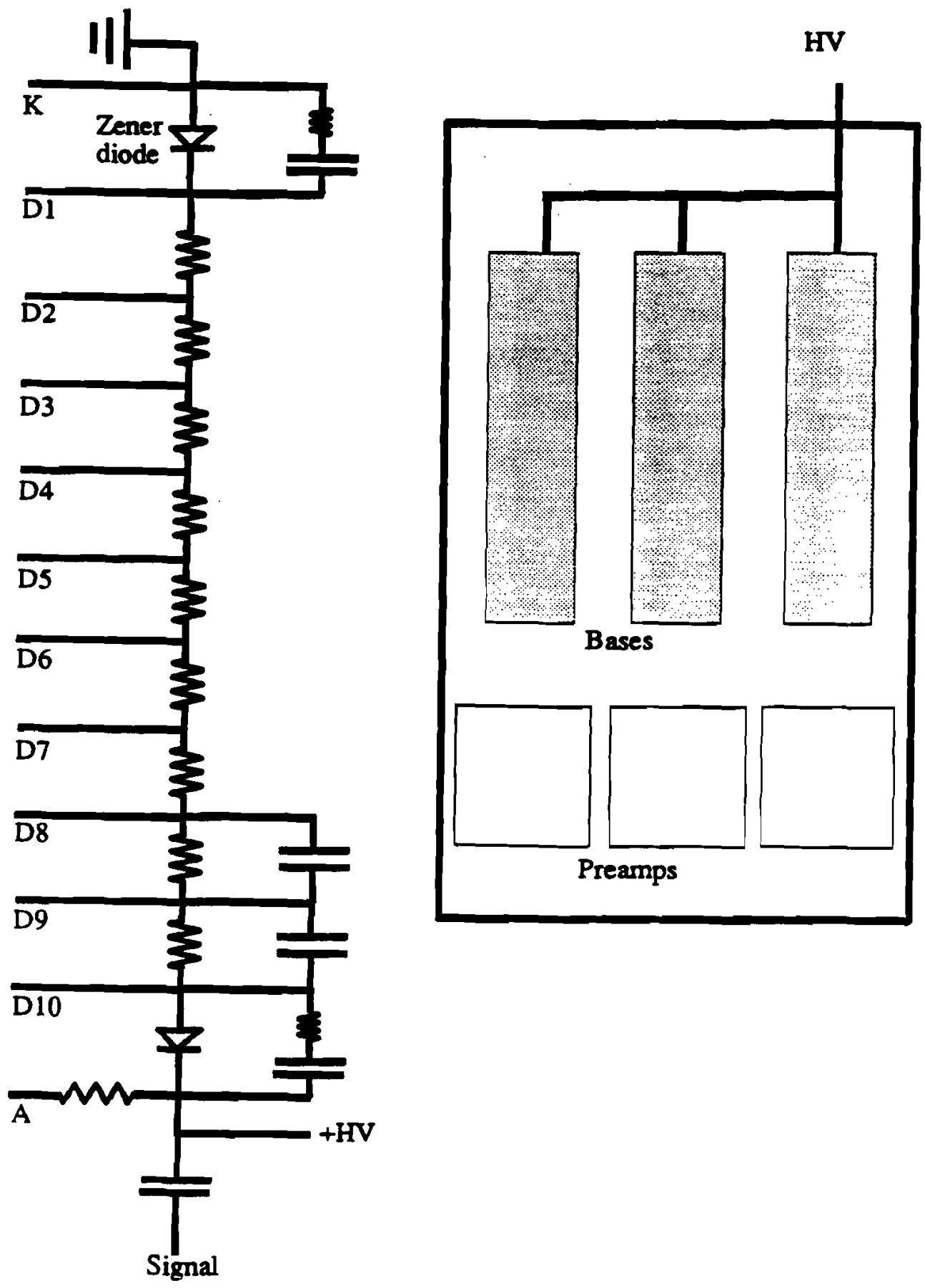

Figure 3.12: Base and preamplifier card design for the ICD. The zener diode on the first stage of the voltage divider protects against accidental catastrophes. The preamp design is similar to that used in the rest of the $D \emptyset$ calorimeter. 
$+750 \mathrm{~V}$ to $+1000 \mathrm{~V}$. To protect against PMT damage due to a potential beam dump, the base is designed to run at low current, at a factor of two less than the maximum anode current rating. The first dynode has a zener diode instead of a resistor so that the voltage drop across that dynode is fixed, regardless of initial input, thereby increasing cathode efficiency. The middle stages of the base serve to kill the tube gain from the design gain of $\sim 10^{6}$ down to $\sim 2 \times 10^{4}$. The resistances on the dynodes (D1-D10) are (in $M \Omega$ ): 0.68 , $1.0,0.68,0.3,0.3,: 68,1.0,1.0,1.0$ for the PM60 tubes and: $1.0,0.68,0.54,0.41,0.30,0.41$, $0.54,0.68,1.0$ for the $R 647$ tubes. The main reason the gain-killing feature was added was to increase the tube life, an important consideration when designing an experiment where access will be limited during long colliding periods.

The preamplifiers used in the ICD are similar to the FET based circuit used in the rest of the $\mathrm{D} \emptyset$ calorimeter. The capacitance has been changed from $10 \mathrm{pF}$ to $22 \mathrm{pF}$ to lower the gain.

\subsubsection{The Laser Calibration System}

A scintillator/PMT detector has inherent differences from unit to unit due to uncontrollable factors such as the tile/fiber couplings or varying quantum efficiencies of the PMT's. An initial testing of each tile was done using a cosmic ray test setup at the UM. The yield for each tile was determined using a cosmic ray test stand. The average yield for all tiles was $\sim 17$ photoelectrons per minimum ionizing particle (pe/MIP). Each PMT was subjected to a set of rigorous tests in a computer-controlled test stand which utilized an LED pulser system [39]. Operating voltage, gain-voltage characteristics and quantum efficiency were determined for each tube. Then tiles and tubes were carefully chosen to form sets of three units with similar input voltages and output signals. These three units were assembled into an aluminum box as a module and tested again with cosmic rays and with a laser setup. Slight mismatching could be fixed by adjusting the extra resistors included in the base design. Severe mismatching could only be solved by choosing new tiles or tubes and reiterating the process.

On-line calibration of the ICD will be accomplished with the use of an innovative laser calibration system [40]. Scintillator aging and PMT gain changes are expected to slowly degrade the ICD response over time. This laser calibration system will provide the primary method of monitoring these changes and maintaining a calibration of the detector through- 
out the duration of the experiment. The advantage of the laser system is that is provides an 'at the source' calibration, i.e., the laser light is brought all the way to the individual tiles where it produces an ionization signal similar to that produced by a particle shower. In this way, the entire detector can be monitored, including tiles, fiber, photomultiplier tubes, electronics and signal digitization.

The system uses a nitrogen ultraviolet (UV) laser. Trunk lines consisting of silica optical fibers carry the laser light $65 \mathrm{~m}$ to four patch boxes located on either side of the two end calorimeters. The patch boxes fan out the laser light to $6 \mathrm{~m}$ jumper cables which take the light to a feedthrough on each ICD module. The jumper cable consists of a single fiber encased in a protective jacket. Viewing the jumper cable from the inside of the ICD module is a one-to-three fanout consisting of a bundle of three silica fibers. The opposite ends of the fibers are embedded in the three scintillator tiles in each ICD box.

The laser is housed in a sturdy aluminum box along with its necessary optics. A filter wheel will be used to vary the amount of light sent to the tiles. The filter wheel system is a set of two wheels with a Tangen Drive wheel indexer that provides reproducible filter positioning over many cycles. By using a variety of neutral density filters, the amount of light seen by a tile can be varied from a few MIPS to 1000 MIP, thus exercising the entire dynamic range of the ICD. A reference photodiode is used to monitor the laser light. 


\section{CHAPTER 4}

\section{THE TEST BEAM PROJECT}

\subsection{Overview of the Test Beam Project}

The developmental phase of a detector often includes some type of test beam run. Generally, a test beam is run in the fixed target mode, where a user-controlled beam of known particles, with specified energy and momentum, is steered towards the module under study. A test beam can be used to test prototype designs of detector elements, as was done for the CC modules. It can also be used to perform in-depth studies of the response of a finalized design as was done for both the CC and EC modules and many of the central detectors. In addition, a test beam can be used to calibrate a detector. By using beams of known energy and particle type, one can study the specific response of the detector to specific input and utilize that information when the detector is exposed to unknown events such as those at the Tevatron.

Most of the initial studies during the R\&D phase of the ICD utilized cosmic rays or radioactive sources. These studies were used primarily to study ICD tile prototype designs. After the final design was chosen, in order to gain a better understanding of the final detector in a realistic $D \emptyset$ setting, we included the ICD in the final $D \emptyset$ test beam run. This run was known as Load II as it was the second in a series of test beam studies. This run focussed on the central calorimeter and on the transition region between the $\mathrm{CC}$ and the EC. This region includes the Intercryostat Detector and the massless gaps. In early 1991, we proposed to include the ICD in the Load II run. This idea posed several interesting problems. A single, small cryostat had been used to contain each of the previous test beam 
loads. The plan for Load II was to build a special cart which would support the included CC and EC modules within the cryostat. The missing cryostat walls would be simulated with steel walls and the intercryostat region (ICR) would be filled with an excluder to simulate the air gap. Our proposal to include the ICD thus required that the scintillator tiles sit in liquid argon. The test beam offered a unique place to study and calibrate the ICD before the actual experiment and so we embarked on a program to determine the feasibility of operating scintillator in LAr.

\subsection{The Test Beam Intercryostat Detector}

\subsubsection{Feasibility Studies}

The impetus for considering placing scintillator in LAr for Load II was a report that a team of Russian scientists had successfully operated scintillator in a LAr environment [41]. A literature search produced only one other such instance, a 1970 bubble chamber experiment which used a scintillator fence for triggering [42]. Using Pilot B scintillator (an early plastic scintillator), a light loss of approximately $30 \%$ was seen at liquid helium temperatures and a loss of approximately $20 \%$ was seen at LAr temperatures. We were thus encouraged to proceed on the general assumption that this idea could succeed. We anticipated several potential problems:

- Survival of scintillator in LAr.

- Survival of fiber in LAr.

- Light losses.

- PMT and electronics survival in LAr.

- Contamination of the LAr by ICD elements.

We wanted to keep things inside the cryostat simple to minimize the $R \& D$ required to put the various elements in liquid argon. We decided to place the PMT's outside the cryostat and instead use long readout fibers to bring the light from the tiles to the PMT's, via sapphire glass interfaces. This decision created its own problems. In the DØ ICD, a 
short ( $0.5 \mathrm{~m}$ ) length of fiber bundle is used. We estimated we would need roughly $3 \mathrm{~m}$ of fibers to traverse the distance from the tiles to the feedthrough port housing the PMT's. The fiber used in the DO ICD is a wavelength shifter with a polystyrene core and a PMMA cladding. The attenuation length of the fiber ensured that we would lose nearly all available light signal from the tile over the distances involved, as will be shown below. We were thus forced into using a non-wavelength shifting (non-WLS) fiber to carry the light over the distance from the tile to the PMT. We also chose to use bundles of non-WLS fiber for the same reason as in the DØ ICD. There were several potentially very tight turns over the distance from tile to feedthrough port. Bundles of thin fibers can be turned through much tighter radii of curvature than a single thick fiber.

Recalling an earlier ICD prototype design, we considered a tile with embedded solid $2 \mathrm{~mm}$ diameter wavelength shifting fibers. These solid core fibers gave a higher light yield than the fiber bundles which were used in the real ICD tiles (but were not flexible enough to fit into the small space available at $D \emptyset$ ). We then took advantage of a new technique [43] in which plastic fibers are spliced together by melting and quickly cooling them after a bond has been formed. This technique enabled us to splice a bundle of non-WLS fibers, with a longer attenuation length, to the solid core fibers. (An additional benefit of using the solid core WLS fibers is that it is much easier to splice a solid core fiber to a bundle than to splice two bundles together.) At this point, we had a general design: solid core WLS fibers spliced to long non-WLS fibers which then carry the light to optical interfaces. The PMT's, safely outside the cryostat, would view the light through the interfaces.

We moved on to the choice of materials. During the R\&D phase of the ICD design, we had studied the possible use of $2 \mathrm{~mm}$ solid core fibers that absorbed in the blue and emitted in the green. These fibers have a PMMA core and a fluorinated polymer cladding. The indices of refraction are 1.6 for the core and 1.4 for the cladding. The absorption spectrum of the fibers was well matched to the emission spectrum of Bicron's BC-400 scintillator, which has a peak emission wavelength of $423 \mathrm{~nm}$. The fibers were supplied by the Optectron Corporation. For the non-WLS fiber, we considered several different clear fibers from PolyOptical. These will be discussed in more detail later.

\section{Qualitative Testing}

There are two major properties of LAr that could affect the light output of the tile: the temperature and the index of refraction. LAr has a much higher index of refraction than 
air (1.22 as opposed to 1.0) and therefore we expected a certain amount of light loss due to a smaller angle of refraction. As LAr is expensive and dificult to use in small quantities, we decided to do initial prototype testing using a small dewar of liquid nitrogen ( $\left.\mathrm{LN}_{2}\right)$. The temperatures of $\mathrm{LN}_{2}$ and $\mathrm{LAr}$ are very similar $\left(\mathrm{N}_{2}\right.$ is liquid at $-85 \mathrm{~K}$ while Argon is liquid at -90K). Likewise, the indices of refraction are not very different (1.22 for LAs, 1.20 for $\mathrm{LN}_{2}$ ). A series of qualitative testing was first done to ensure the mechanical durability of the various detector materials. Small samples of the scintillator, WLS fiber and nonWLS fibers were immersed in $\mathrm{LN}_{2}$ for $5 \mathrm{~min}, 20 \mathrm{~min}$ and overnight. The samples were carefully examined, macroscopically and under a microscope, before and after immersion. Results were very encouraging. The scintillator samples maintained proper color, opacity and showed no signs of crazing or other mechanical duress. The fiber samples aiso showed no signs of degradation and maintained their general light transmission properties.

As a next step, samples consisting of WLS fiber embedded in a small block of scintillator were tested. Samples were prepared using either $5 \mathrm{~min}$ epoxy or optical epoxy (24 hr curing time). These mini tiles were also immersed for short intervals and overnight. As before, there were no signs of mechanical duress and the glue joints showed no signs of degradation. Overall, there did not appear to be any short-term survival problems with operating in $\mathrm{LN}_{2}$. In addition, repeated cycling between the temperature extremes did not appear to adversely affect either scintillator or fiber. However, we did find it necessary to slowly cool the scintillator to avoid shattering.

\section{The Non-Wavelength Shifting Fiber}

The choice of non-WLS fiber was based on several factors. The fiber had to transmit in the appropriate wavelength range; the attenuation length had to be long enough to minimize light loss over the length of the fiber from tile to feedthrough port; space limitations required a fairly tight radius of curvature for the fibers as they exited the aluminum boxes on the way to the optical interfaces. Cost was also a factor.

For the non-WLS fiber, we considered several different plastic fibers manufactured by PolyOptical Products, Inc. PolyOptical was providing the WLS fiber used in the D $\emptyset$ ICD tiles and had proved to be a reliable and competitively priced supplier. Plastic was a necessary choice as we needed to be able to melt the fiber in order to splice it to the WLS fibers. The three fiber types explored were (i) polystyrene fibers; (ii) industrial grade acrylic fibers; and (iii) optical grade acrylic. The polystyrene fibers had a polystyrene (PS) core with a 
polymethyl methacrylate (PMMA) cladding. Its attenuation of $1200 \mathrm{~dB} / \mathrm{km}$ at $675 \mathrm{~nm}$ was not very good; however, the low cost of the fiber made it attractive. Also, the PolyOptical data sheet claimed "cryogenic" temperatures were allowable with the PS/PMMA fiber.

The acrylic fibers had a PMMA core with a fluorinated polymer (FP) cladding. The attenuation was much better; a nominal $250 \mathrm{~dB} / \mathrm{km}$ at $650 \mathrm{~nm}$ for the industrial grade quality and $200 \mathrm{~dB} / \mathrm{km}$ at $650 \mathrm{~nm}$ for the optical grade fiber. The difference between optical and industrial grade fiber became apparent at shorter wavelengths. At the operating wavelength for the WLS fiber ( $\sim 500 \mathrm{~nm}$ ), the attenuation of the optical grade was still $\sim 200 \mathrm{~dB} / \mathrm{km}$ while the industrial grade's rose to $400 \mathrm{~dB} / \mathrm{km}$.

Some 'back of the envelope' calculations were used to further narrow down the choice of fiber. An estimate of the distance from where the tiles would sit in the test beam cryostat to the feedthrough port where the PMT's would sit was approximately $3 \mathrm{~m}$. Over that distance, the light loss for the PS/PMMA fiber would be greater than $95 \%$. For the industrial grade PMMA/FP fiber, the light loss would be approximately $70 \%$ and for the optical grade, approximately $45 \%$. In the case of the DØ ICD tiles, the light output averages around 20 photoelectrons per MIP (pe/MIP) at the PMT. The signal can be broken into two parts. The first part is the signal from the tile and the length of WLS fiber that is actually embedded into the tile. The second part is a signal loss as the light travels through the extra length of WLS from where it exits the tile to the PMT. How much attenuation is this? The extra fiber distance is approximately $0.5 \mathrm{~m}$. The attenuation of the WLS fiber is no better than that of the PS fiber. Thus, the light loss is around $50 \%$ in that extra length of WLS fiber. So the original signal from the tile and embedded WLS fiber, at the point where they would be spliced, can be estimated to be $40 \mathrm{pe} / \mathrm{MIP}$. Assuming a comparable signal in the test beam tiles, we could expect a final signal of about 2 pe/MIP using the PS/PMMA fiber; 12 pe/MIP using the industrial grade PMMA/FP fiber; and 20 pe/MIP using the optical grade PMMA/FP fiber.

These calculations convinced us that we were going to have real signal loss problems if we tried to use a cheap fiber. Therefore, we decided to use the optical grade PMMA/FP fiber and ordered a small spool for testing purposes. However, we also ordered a small spool of the PS/PMMA for comparison purposes, as cryogenic temperatures were acceptable for that fiber. 


\section{The Splicing Technique}

We ordered 0.02 inch diameter fibers of each type. After its arrival, we worked on development of the splicing technique based on that described in a recent Fermilab preprint article [43]. In this technique, single fibers are inserted into glass tubes. A resistive wire is wrapped once around the tube and current is applied until the fibers melt and are completely fused. After a few seconds cooling time, the glass tube can be removed and the resultant fiber joint has an optical efficiency approaching $95 \%$. Our situation was slightly different. We wanted to splice a bundle of many fibers to a single $2 \mathrm{~mm}$ fiber. The $2 \mathrm{~mm}$ fiber was thicker than had been tried by the Fermilab group, which had fused $1 \mathrm{~mm}$ fibers to $1 \mathrm{~mm}$ fibers. By trial and error, we arrived at a working system to splice the solid core WLS fibers to the bundle of non-WLS fibers.

A schematic of the splicing setup is shown Figure 4.1. Eleven of the 0.02 inches nonWLS fibers were slid into one end of a short length ( 1.5 inches) of glass tubing. The WLS fiber, polished flat, was slid into the other end. A $2.4 \Omega$ resistive wire was slid over the tubing. Three loops of the wire were used to surround the fusing point. A $12 \mathrm{~V}$ power supply was used to heat the wire. As the wire heated, the fiber and bundle were slowly kept in motion, rotating slightly and moving them back and forth in the tubing. This helped to distribute the heat evenly as the thicker fiber and bundle took longer to heat and melt thoroughly. After approximately 30 seconds, the fibers would begin to fuse. At this point, pressure was applied to push the fiber and bundle firmly together so that all eleven fibers in the bundle would fuse to the solid core fiber. The fiber and bundle joint expanded slightly to fill the tube. After another few seconds, the joint was about $1 / 2$ inches long and completely fused, as shown in Figure 4.1. Shutting off the power supply, the resistive wire was quickly slid off the glass to ensure no further melting. After a short ( $\sim 30$ seconds) cooling time, the glass tubing could usually be easily removed. However, we found that sometimes an attempt to remove the glass would cause enough stress to break the joint or would scratch the cladding of the thicker joint area. This seemed to be related to the room temperature. In hotter weather, it was more difficult to safely remove the glass, even after several minutes of cooling. To solve this problem, we used a shot of cold water on the glass tubing to quickly cool the joint and glass. The fused joint between the fiber and bundle was found to be susceptible to shear stress but withstood tensile stress well. When assembling the tiles, a drop of optical glue was added to each joint to decrease the danger of shear 
Schematic of Splicing Setup
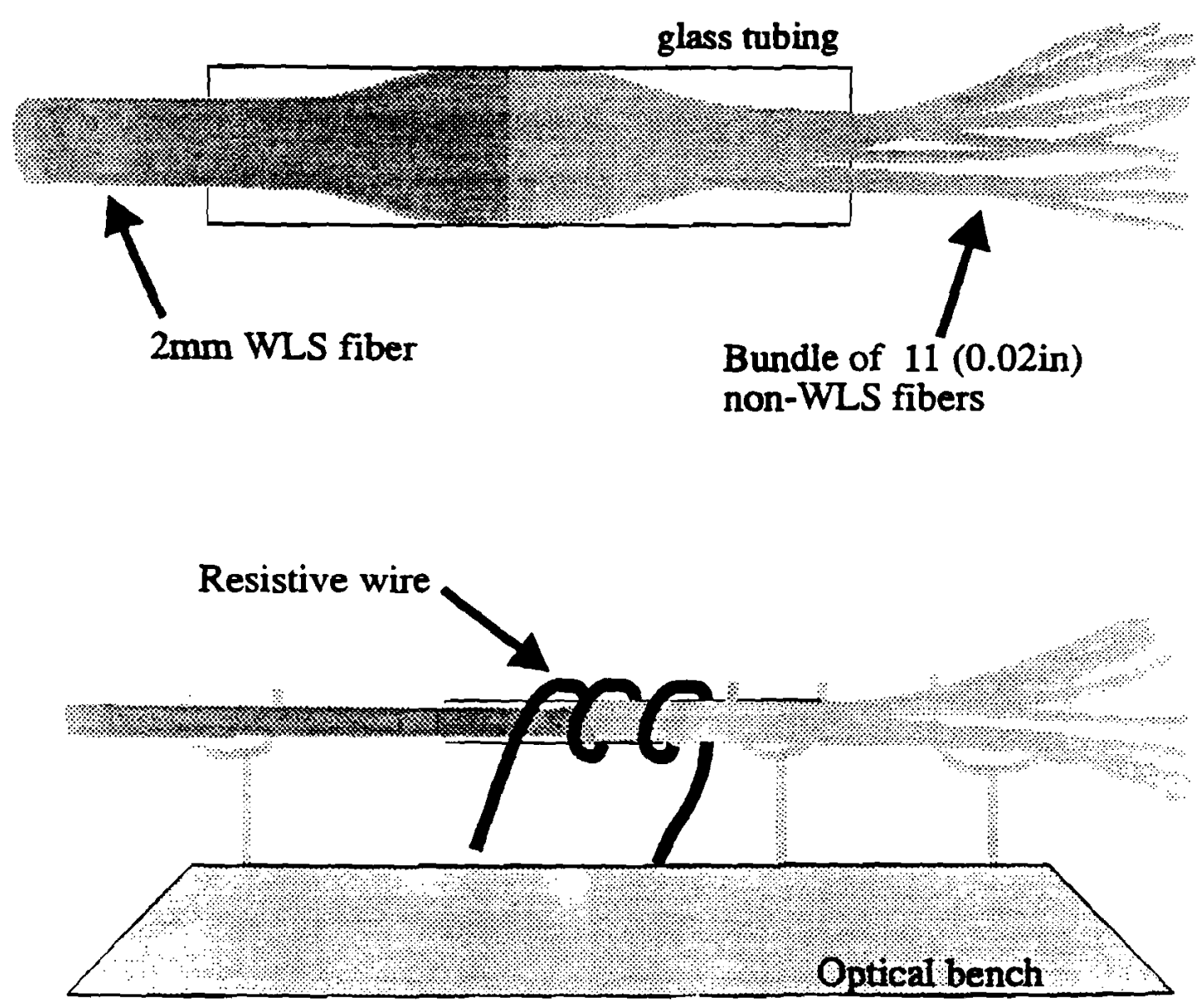

Figure 4.1: A schematic of the setup used in the splicing technique. An optical bench was used to keep the solid core fiber and the fiber bundles straight. 
breakage.

As with the other samples of material, we did a series of qualitative testing on the spliced fibers. Small lengths of green fiber were spliced to a bundle of non-WLS fiber, both PMMA/FP and PS/PMMA, and these samples were immersed in $L_{2}$. Both short term and overnight tests were done. Even without adding glue to the joint, the splice withstood the cold conditions well. There was no sign of breakage or other mechanical stress.

\section{IN2 Testing Setup}

Since many of the prototype tests were performed using a common system, it would be instructive to describe the basic setup. Figure 4.2 is a schematic drawing of the test setup. Cold tests were performed in a 15 inch tall by 6 inch diameter dewar. The lid of the dewar was 1 inches thick polystyrene foam. Holes were punched through the lid for $\mathrm{LN}_{2}$ input, light input, light output and other signals. A piece of black rubber was placed over the lid to improve the insulation and light-tightness. Malleable putty was used to seal the lid-dewar joint and helped to improve the insulation and light-tightness.

A system of three resistors [44] was set up to indicate the level of the $\mathrm{LN}_{2}$ in the dewar. When filling the dewar, we first allowed small amounts of gaseous nitrogen to leak in and slowly cool the dewar and contents for about one hour. After a stable temperature was reached, the nitrogen would begin to condense and fill the dewar. As the level of $\mathrm{LN}_{2}$ in the dewar reached each of the resistors, its resistance would rapidly increase from an initial resistance of $75 \mathrm{k} \Omega$ to $135 \mathrm{k} \Omega$. In this way, one could judge, for instance, if signal changes were related to the liquid nitrogen reaching a particularly significant level (as will be discussed later) or when the dewar was full.

The light source for the tests was either an ultraviolet lamp operating around $400 \mathrm{~nm}$, used to generate light in the scintillator, or a green LED with a wavelength similar to that of the WLS fibers, used to test the fibers. An aluminum housing was designed to hold either the lamp or the LED. Viewing the light source, at a distance of about $10 \mathrm{~cm}$, was a reference photodiode (PD). Directly above the PD was a PVC collar which was used to hold the input fiber bundle. The input fiber bundle could either be the fiber bundle under test, as in the radius of curvature tests for the non-WLS fibers, or it could be bundle used to carry the UV light to a scintillator tile, as in the prototype tile testing. A Hamamatsu R647-1 photomultiplier tube, running off a standard base, was used to measure all output signals. All output signals have been normalized to the initial reference PD signal. 


\section{Schematic of $\mathrm{LN}_{2}$ Feasibility Test Setup}

\section{Light Source}
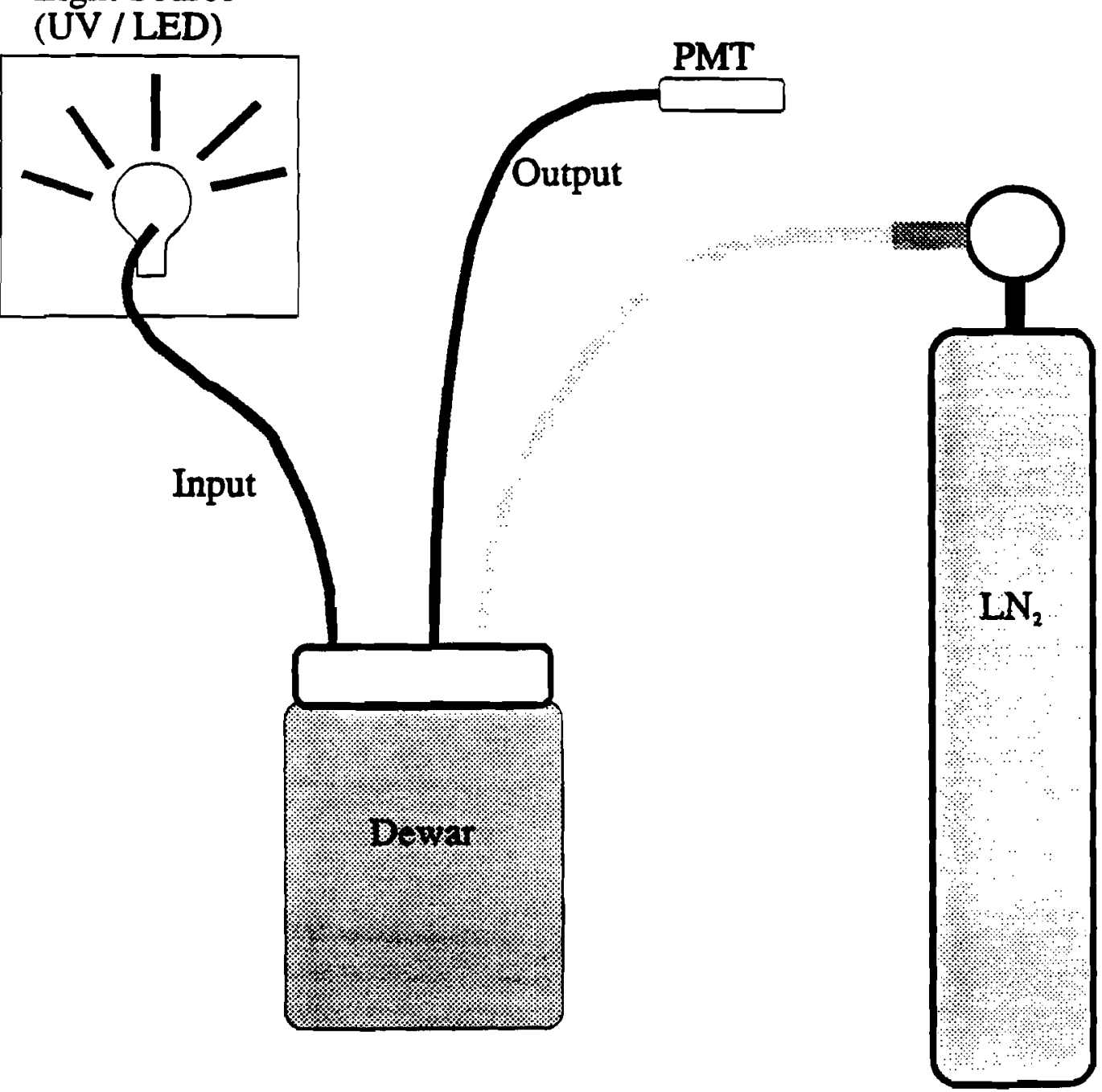

Figure 4.2: A schematic of the liquid nitrogen test setup. 


\section{Radius of Curvature Testing of the non-WLS fibers}

Other than attenuation length, the most important criterion for the non-WLS fiber was the minimum radius of curvature allowed. We anticipated several places where the fibers would have to bend through a fairly small radius of curvature to traverse the distance from tile to PMT. Nominally, the minimm allowable radius of curvature is $15 \times$ (diameter) for the PMMA/FP fiber and $20 \times$ (diameter) for the PS/PMMA fiber. For testing purposes, we ordered 0.02 inches diameter fiber. Therefore, the minimum bend radii are 0.3 and 0.4 inches for the PMMA/FP and PS/PMMA fibers, respectively. We decided to check the nominal limits and investigate how operating in $\mathrm{LN}_{2}$ affected a tightly curved bundle of fibers.

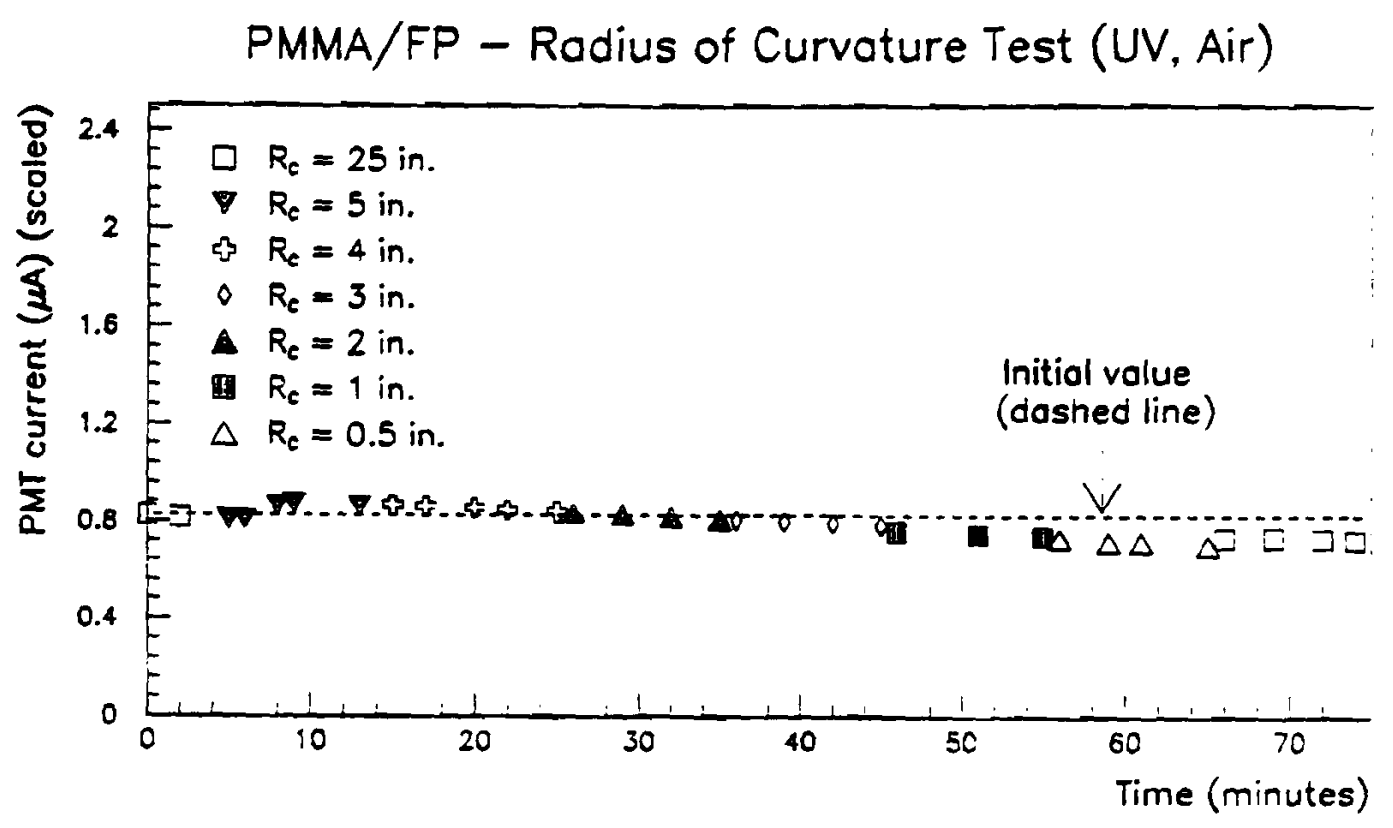

Figure 4.3: The initial radius of curvature test for PMMA/FP fiber, using UV light. The PMT response is plotted as $R_{C}$ is changed from a relared position of $R_{C}=25$ inches to a minimum of $R_{C}=$ 0.5 inches.

The first test was a simple room temperature test, varying the radius of curvature, $\mathrm{R}_{\mathrm{C}}$, of an 80 inch bundle of five PMMA/FP or PS/PMMA fibers. The fiber bundles were encased in black teflon tubing and covered with black felt to improve light-tightness. The light source was the UV lamp. The radius of curvature was manually decreased from a relaxed position, $R_{C}=25$ inches, to $R_{C}=5$ inches and then decreased to $R_{C}=4$ inches, 
$R_{C}=3$ inches, $R_{C}=2$ inches, $R_{C}=1$ inches, $R_{C}=0.5$ inches. The fiber bundle was left in each position for 10 minutes. The results, normalized to the reference photodiode, are shown in Figure 4.3 and Figure 4.4. The light loss is $~ 2 \%$ for the PS/PMMA fiber. The PMMA/FP fiber exhibited a constant decrease in signal, dropping to $\sim 12 \%$ at $R_{C}=$ 0.5 inches.

\section{PS/PMMA - Radius of Curvature Test (UV, Air)}

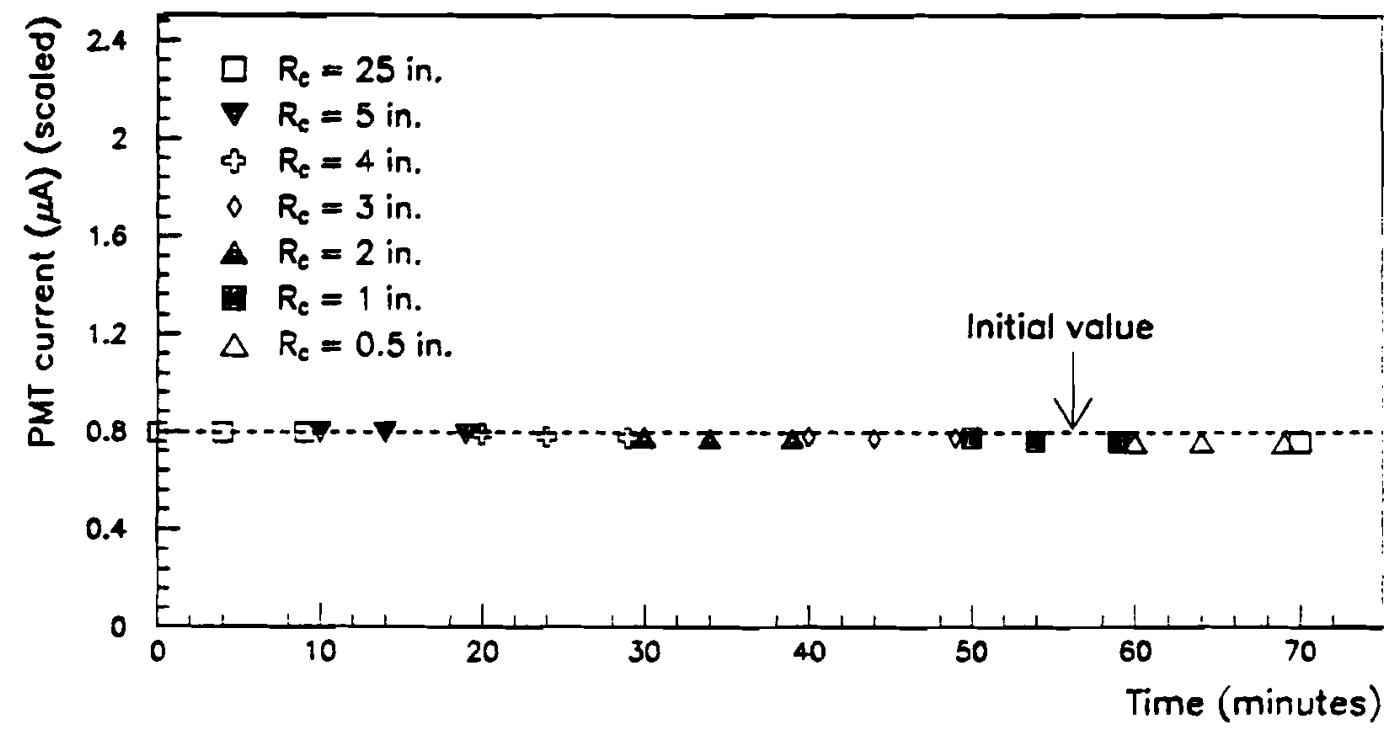

Figure 4.4: The radius of curvature test for PS/PMMA fiber, using UV light. The PMT response is plotted as $R_{C}$ is changed from a relaxed position of $R_{C}=25$ inches to a minimum of $R_{C}=$ 0.5 inches.

As these plastic fibers do not generally transmit well in the UV, the same test was later done using a green LED to more closely simulate the light which would be transmitted by the fiber. Using the LED, we first did a baseline test to check the long-term signal from a bundle of unstressed fibers. A bundle of fibers was taped to the table top and covered with black felt to ensure light-tightness. For $R_{C}=22$ inches, there was a very slight decrease of $0.5 \%$ in the signal over a two day period. For $R_{C}=12$ inches, there was a decrease of $1 \%$ over a one day period. These results are shown in Figure 4.5.

We then repeated the radius of curvature test for the PMMA/FP fiber in air, using the green LED as the light source. This result is shown in Figure 4.6. The radius of curvature was varied from 12 inches to 1 inches. The light loss is $1 \%$ at $R_{C}=2$ inches, but increases to $7 \%$ at $R_{C}=1$ inches. 


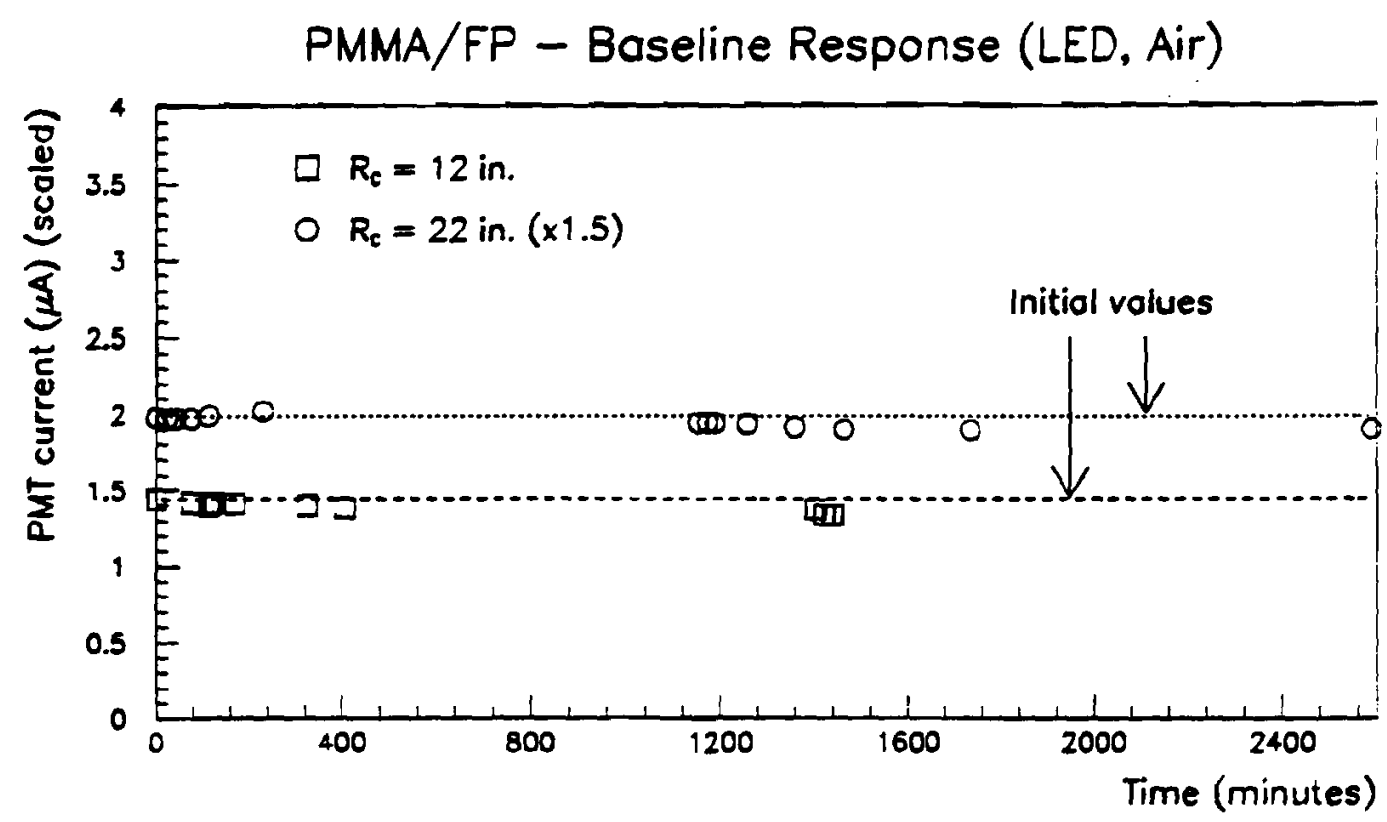

Figure 4.5: Signal from a bundle of PMMA/FP fibers, using the green LED as the light source. The PMT response is plotted for $R_{C}=22$ inches and $R_{C}=12$ inches.

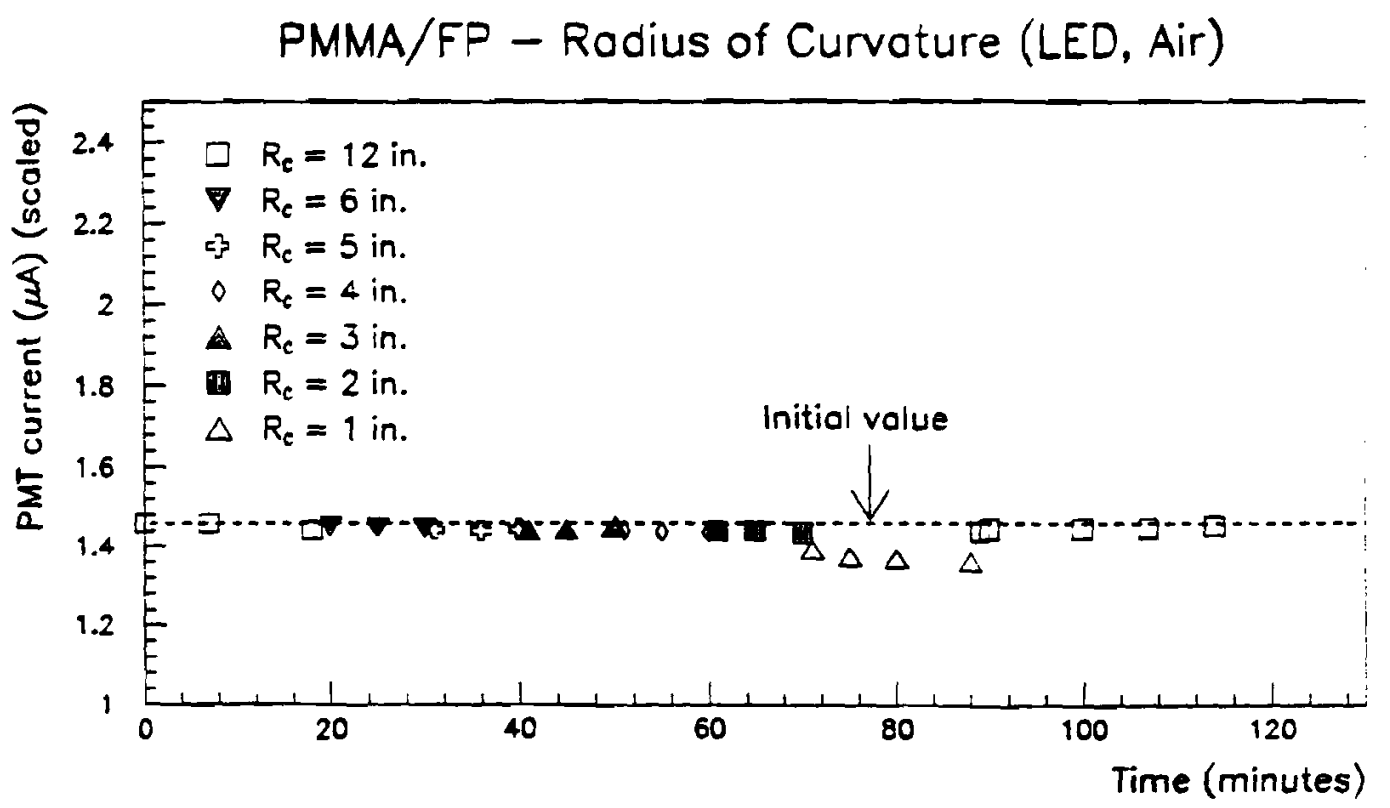

Figure 4.6: The radius of curvature test for PMMA/FP fiber, using the green LED. The PMT response is plotted as $R_{C}$ is changed from a relaxed position of $R_{C}=12$ inches to a minimum of $\mathrm{R}_{\mathrm{C}}=1$ inches. 
Due to the slightly higher light loss of the PMMA/FP fiber, we decided to do additional testing to look at possible long term effects under fairly tight radii of curvature. An acrylic frame was made to hold the fiber bundles at a constant $R_{C}=2$ inches. This frame was inserted into the test dewar. The PMMA/FP fiber bundle was tested using the green LED and both in air and in $\mathrm{LN}_{2}$. In air, the light output of the fiber bundle decreased $\sim 1 \%$. Under cold conditions, there was a definite drop in light output of $\sim 5 \%$ as the fiber bundle was cooling. After a stable temperature was reached, the light output recovered to more than the original level and remained at that higher level. There were several potential causes for this increase, such as temperature effects on the PMT and the reference photodiode or instability of the UV lamp. However, the important result was that we saw no significant long-term light decrease due to the fiber bundle radius of curvature. The $\mathrm{LN}_{2}$ results are shown in Figure 4.7. The level-sensing resistors were used to determine when the dewar was full, indicated on the plot by the 'cold' pointer. The test was maintained for three days. Overnight, the dewar would lose about $30 \%$ of its $\mathrm{LN}_{2}$. Therefore, the dewar was refilled each day.

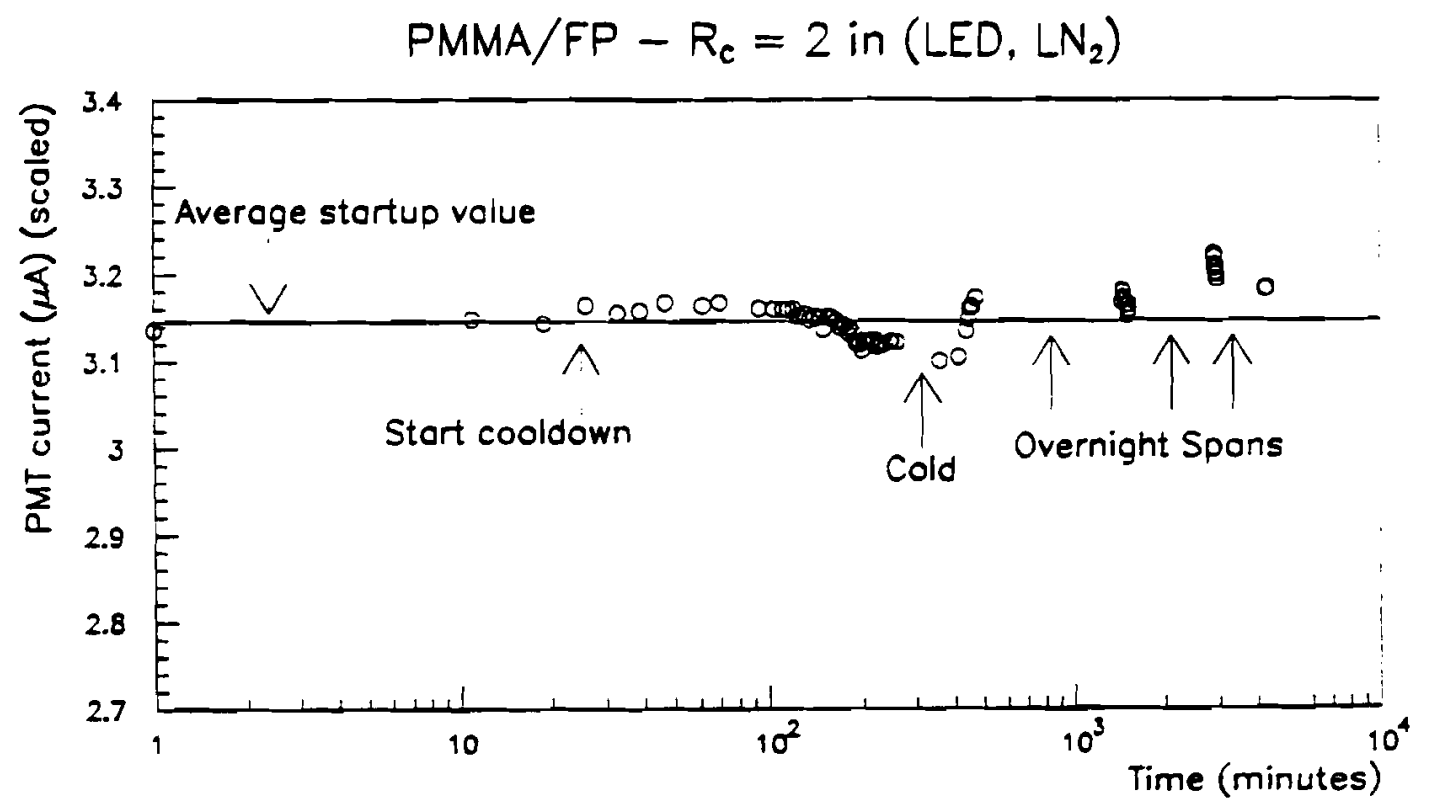

Figure 4.7: Using the green LED light, radius of curvature test for the PMMA/FP fiber bundle in $\mathrm{LN}_{2}$.

Finally, a test was done on a very long length of PMMA/FP fiber bundle. Early in our testing, it was not clear how many times a bundle might have to turn through a tight radius 
while negotiating the distance from tile to PMT. By testing a long bundle and coiling it many times, we could get an idea of the cumnlative effect. The fiber bundle was $25 \mathrm{ft}$ long and was coiled into the dewar. The coils had an average radius of curvature of 2-3 inches. The green LED was used for this test. We noticed it took a long time for a stable initial value to be reached. So we first allowed the bundle to sit overnight in air. The fiber bundle was then cooled down and allowed to sit overnight. No appreciable light loss was observed over the several day span of the test. These results are shown in Figure 4.8.

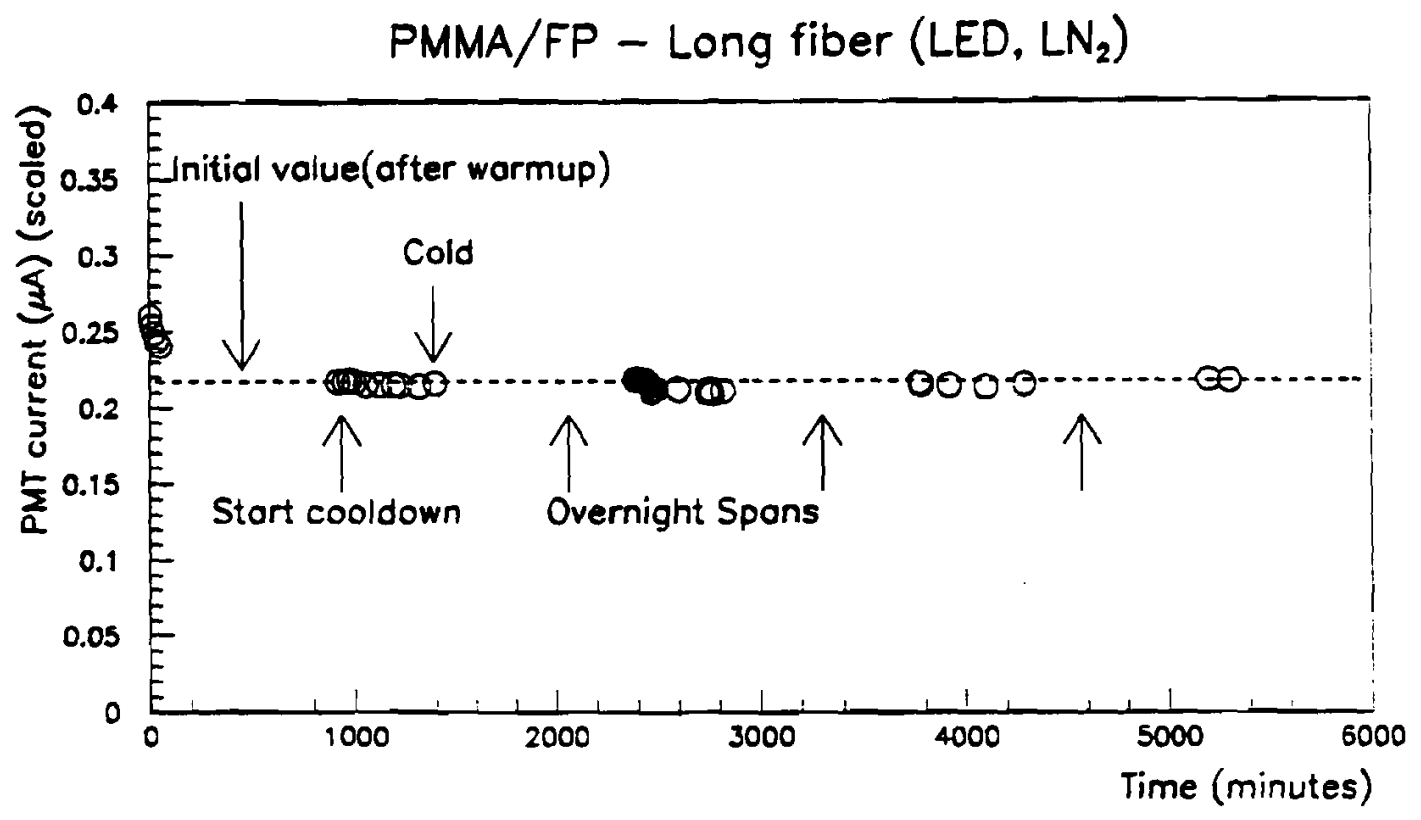

Figure 4.8: Long term radius of curvature test of the PMMA/FP fiber bundle, using the green $\mathrm{LED}$, in $\mathrm{LN}_{2}$.

\section{Tile Prototype Tests}

The most critical test was the test of the prototype test beam tile. This prototype tile was 2 inches by 3 inches, with two fiber grooves. A bundle of polystyrene fibers carried the UV light into the dewar and was glued onto the face of the prototype tile. The non-WLS PMMA/FP bundle from the tile also exited through the lid of the dewar and was viewed by the R647-1 PMT. The tile test setup is shown in Figure 4.9.

Several test runs were performed. The results of the final long-term study of a tile using PMMA/FP bundles are shown in Figure 4.10. The overall drop in signal as the tile was cooled and covered with liquid nitrogen was approximately $40 \%$. 


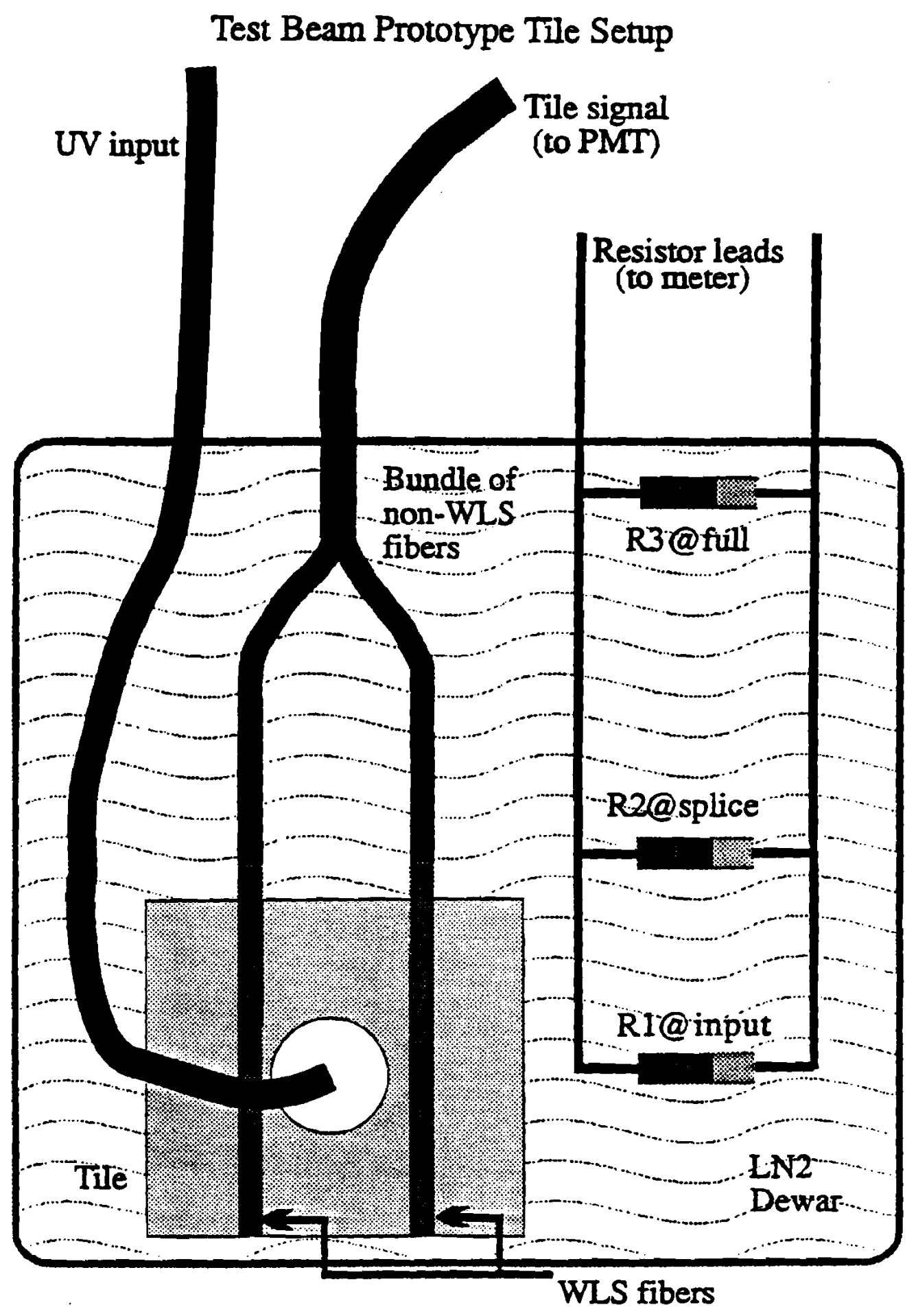

Figure 4.9: The setup for the prototype test beam tile test. The positions of the three level-sensing resistors with respect to tile are shown. 
The three $\mathrm{LN}_{2}$ level-sensing resistors were placed at specific levels so we could determine if a signal drop was correlated with any specific event. The first resistor was placed level with the UV light input bundle. This bundle was located in the center of the tile. The second resistor was placed level with the splice between the WLS green fibers and the nonWLS fiber bundles. The third resistor was placed near the top of the dewar to indicate when it was full. The dewar contents were cooled for about one hour before the $\mathrm{LN}_{2}$ began condensing. As one can see, the light output from the tile began to drop before the $\mathrm{LN}_{2}$ level reached the UV input. Thus, it actually began to drop as soon as the $\mathrm{LN}_{2}$ level reached the bottom of the tile, as one would expect from the difference in the indices of refraction of air and $\mathrm{LN}_{2}$ (as discussed below). The tile response continued to drop until the tile was completely immersed. One can see that the response levels off at the second resistor. This is at the splice, which is just outside the top of the tile. There is no additional drop in response associated with the splice. The tile response remained constant overnight and into the next day as shown.

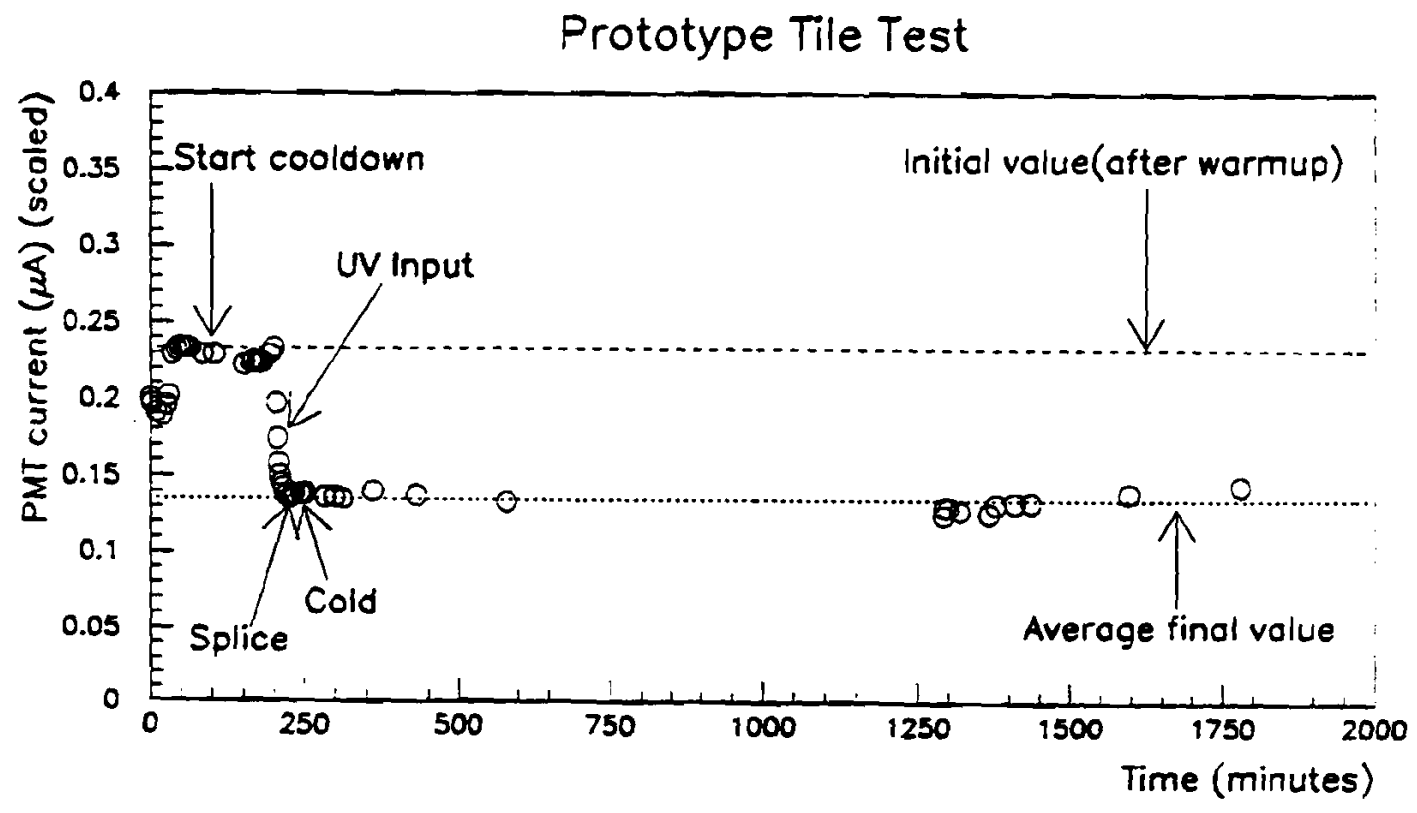

Figure 4.10: Response of a prototype test beam tile as a function of time. The test dewar was slowly filled with liquid nitrogen and important instances are noted.

The overall signal loss of $40 \%$ initially seemed higher than expected. However, this loss can be easily understood by taking into account the difference in the indices of refraction 
of air and $\mathrm{LN}_{2}$ (or LAr). Consider how the signal is produced. Ionization photons are produced when the scintillator is excited by the incident UV light. These photons bounce around inside the tile until one of three things happen: they enter a fiber and are wavelength shifted inside the fiber to produce the signal; they hit the tile surface and escape into the outside fluid medium; or they bounce so often and travel so far that all their energy is essentially lost.

Only the second possibility is affected by the increased index of refraction of the medium. The probability for escape is governed by the critical angle at the scintillator/fluid interface as those photons which undergo total internal reffection may hit a fiber on a later bounce. The critical angle, $\theta_{e}$ is given by:

$$
\sin \theta_{c}=\frac{n_{0}}{n_{i}}
$$

where $n_{0}$ is the index of refraction of the outer medium (either air or $L N_{2}$ ) and $n_{i}$ is the index of refraction of the initial medium (scintillator). For air, $\theta_{c}=\sin ^{-1}(1.00 / 1.58)=$ $39.3^{\circ}$. For $\mathrm{LN}_{2}, \theta_{c}=\sin ^{-1}(1.20 / 1.58)=49.4^{\circ}$. Consider a point on the interface as shown in Figure 4.11. Those photons which hit the interface at an angle less than $\theta_{c}$ will escape and contribute to the signal loss. The volume for photons which do not escape is given by

$$
\begin{gathered}
V=0.5 \int_{0}^{2 \pi} d \phi \int_{0}^{R} r^{2} d r \int_{0}^{80^{\circ}-\theta_{c}} \sin \theta d \theta \\
V=\frac{\pi R^{3}}{3}\left(1-\cos \left(90^{\circ}-\theta_{c}\right)\right) \\
\Rightarrow V_{a i r} \propto 1-\cos \left(50.7^{\circ}\right) \propto 0.367 \\
\Rightarrow V_{\mathrm{LN}_{2}} \propto 1-\cos \left(40.6^{\circ}\right) \propto 0.241 \\
\Rightarrow \frac{V_{\mathrm{LN}}}{V_{\text {air }}}=0.657 .
\end{gathered}
$$

The volume where the photons could potentially be captured is therefore nearly $35 \%$ less when the tile is in $\mathrm{LN}_{2}$ than when it is in air. This is, of course, a first order calculation that does not fully take into account the tile corners and other geometrical factors. However, it does show that the major factor in the light decrease is due to the different indices of refraction of the outside medium.

To make up for some of the signal loss, we made two changes to the tile design. First, we increased the thickness of the scintillator from the nominal ICD thickness of 0.394 inches $(1.0 \mathrm{~cm})$ to 0.75 inches. Second, we increased the depth of the groove and embedded two fibers in each groove. At one point during our R\&D phase, we studied the effect of increasing 
Fluid (air or $\mathrm{LN}_{2}$ )

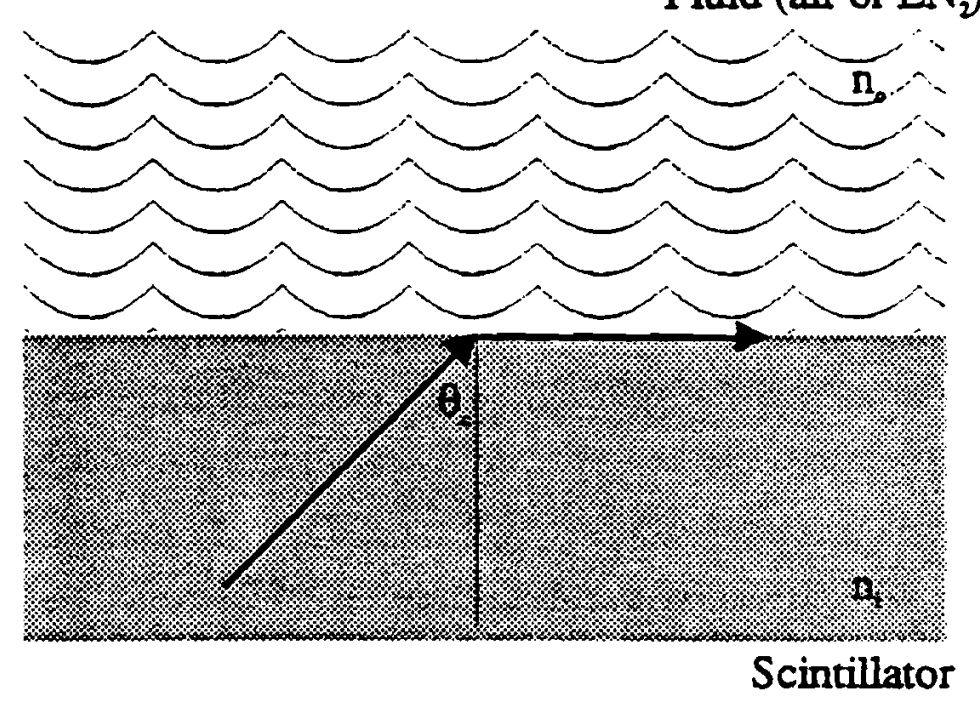

Figure 4.11: Interface between the tile and outer medium (air of $\mathrm{LN}_{2}$ ). 
the depth of the fiber groove. We found a non-negligible benefit to increasing the groove depth, on the order of $25 \%$. This effect can be understood when considering the increased angle subtended by the fiber in a deeper groove. Based on this testing, we decided to double the depth of the groove. We then embedded two of the green WLS solid core fibers in each groove, each fiber spliced to a bundle of the non-WLS fibers.

The fiber groove depth work was later studied in detail by a senior honors student [45]. Using a Monte Carlo simulation to mimic the path of photons in scintillator and fiber, he studied the effect of fiber depth on light output. He found that light output increased as a function of fiber depth, up to an optimum fiber depth. The optimum fiber depth was found to be at $89 \%$ of the tile depth. A special Monte Carlo run was done using the dimensions of one of the test beam tiles. The tile depth was 0.75 inches. By doubling the fiber depth from the nominal 0.08 inches to 0.16 inches, the light output of the tile increased by $19 \%$, in reasonable agreement with the experimentally observed increase.

\subsubsection{Contamination Studies}

One of our primary concerns was the possibility of contamination of the liquid argon due to the various elements in the ICD. The calorimeter response is extremely sensitive to the level of oxygen contamination in the LAr. To study this potential problem, we enlisted the aid of the University of Rochester [46] group who maintained a contamination test cell at Fermilab and at Rochester. The test cell consisted of a miniature stack of uranium and readout layers in a 5 liter cryostat. The response of the test cell to both alpha and beta radiation emitters was monitored before and after addition of potential contaminants. We prepared test samples of each of the ICD elements. Each sample was made up of approximately 10 times the amount by volume which would be in the actual test beam cryostat, scaled down by the ratio of LAr in the test beam cryostat (10000 liters) to that in the test cryostat. Samples included: scintillator, WLS fiber, non-WLS fiber and optical epory. Known safe materials, such as aluminum and teflon, were not included.

Initial test results from the Fermilab test cell gave us a real scare. Contamination levels were so high as to ensure the ruin of the test beam project. However, a leak in the test system was found to be the source of the contamination and we prepared a second set of samples to be tested at the Rochester cryostat. These second results relieved the fears of contamination due to the ICD. At the test beam operating voltage of $2.5 \mathrm{kV}$, there was no 
measurable decrease in the test cell response to either radiation. The oxygen concentration increased at the rate of $0.007 \mathrm{ppm} /$ day. Extrapolating this rate to the volumes at the test beam, an upper limit of $0.15 \mathrm{ppm} /$ year oxygen was determined. Given the short duration of the test beam run, it was decided this rate was not enough to affect the calorimeter performance and we were given the go-ahead to put the ICD in the test beam cryostat.

For a final determination of the LAr contamination rate due to the ICD, we can consider the actual contamination rate at the test beam. Over the 200 day duration of the Load II sun, the alpha response dropped $0.2 \%$, which corresponds to a $0.07 \%$ drop in the calorimeter response [47]. This can be compared to the contamination rate of the previous test beam run (1990 Load I studied the ECEM in detail), in which there were no scintillator tiles. During the 100 day run of the Load I run (using the same volume of LAr), the alpha response dropped $<0.1 \%$, corresponding to a $0.03 \%$ drop in the calorimeter response [48]. The extra $0.01 \%$ decrease in response in Load II implies a yearly decrease of $0.018 \% /$ year. The amount of scintillator in the 10000 liter test beam cryostat was $\sim 11500 \mathrm{~cm}^{3}$. Assuming all the extra contamination was due to the ICD, one finds an upper limit on the decreased rate of response of the calorimeter to be $0.16 \%$ per (yr $/ \mathrm{kl}-\mathrm{LAr} /\left(\mathrm{m}^{3}\right.$-scintillator $)$ ).

\subsubsection{Final Design}

The final design for the test beam ICD tiles consisted of BC. 400 scintillator with two layers of embedded solid core wavelength shifting fibers. The WLS fibers were spliced to bundles of non-wavelength shifting fibers which took the light from the tiles to sapphire glass windows (with a $\geq 95 \%$ transmission rate), embedded in a feedtbrough port. Outside the windows, the PMT's viewed the bundles of fibers. The design differences between the D $\emptyset$ ICD and the TB ICD are summarized in Table 4.1. The electronics for the test beam setup was essentially the same as that used in the real detector. Due to a delay in the design of the ICD pre-amps, we decided to use the calorimeter pre-amps for the test beam ICD tile readout. We used the $10 \mathrm{pf}$ pre-amps (rather than the standard calorimeter $5 \mathrm{pf}$ type) boosting the gain by a factor of two. However, since the test beam tile yieids were lower than the real ICD tiles, the increase in gain was not expected to affect our dynamic range. 


\begin{tabular}{|l|c|c|c|}
\hline Detector: & DQ & \multicolumn{2}{|c|}{ TB } \\
\hline Environment: & Air & \multicolumn{2}{|c|}{ LAr } \\
\hline Scintillator: & BC-414 & \multicolumn{2}{|c|}{ BC-400 } \\
\hline Fiber: & WLS & WLS & non-WLS \\
Material: & PS/PMMA & PMMA/FP & PMMA/FP \\
Diameter: & $100 \mu \mathrm{m}$ & $2 \mathrm{~mm}$ & $0.01 \mathrm{in}$ \\
Number/groove: & $\sim 200$ & 2 & $22(11 /$ WLS fiber $)$ \\
Length: & $\sim 65 \mathrm{~cm}$ & $12-20 \mathrm{~cm}$ & $\sim 3 \mathrm{~m}$ \\
\hline Optical Interface: & None & \multicolumn{2}{|c|}{ Sapphire glass plates } \\
\hline
\end{tabular}

Table 4.1: A comparison of the two detectors, DØ ICD and TB ICD.

\subsubsection{Production of the Test Beam ICD Modules}

To ensure that we did not add any contaminants to the LAr, we took extra care in the production process. A clean room was constructed of unistrut and plastic in the production lab. Fibers and tiles were given an isopropyl wash and rinsed in sterile water. The optical epoxy had been cleared with the contamination study, but the black tape, which was used to wrap the $D \emptyset$ tiles, was not allowed. Therefore, we first wrapped the TB tiles in the teflon film (also used for the DØ tiles). To help secure the tefion film and to improve the light-tightness of the tiles, we wrapped the tiles with a grease-free aluminum foil. The fiber bundles were encased in tefion tubing (washed and rinsed) before being placed in their Al boxes (also washed and rinsed). For transport to FNAL, the boxes were wrapped in a double layer of clean plastic bags.

All 30 of the test beam tiles were tested in the cosmic ray test stand to determine their minimum ionizing response. Before beginning the testing, we chose a minimum acceptable response of $10 \mathrm{pe} / \mathrm{MIP}$. The MIP response for each tile is summarized in Table 4.2. The average response for all tiles was $13 \mathrm{pe} / \mathrm{MIP}$. 


\begin{tabular}{|c||c|c|c|c|c|c|}
\hline$\phi \backslash \eta$ & $0.8-0.9$ & $0.9-1.0$ & $1.0-1.1$ & $1.1-1.2$ & $1.2-1.3$ & $1.3-1.4$ \\
\hline \hline 31 & 13 & 15 & 18 & 14 & 10 & 13 \\
\hline 32 & 13 & 13 & 10 & 17 & 13 & 22 \\
\hline 33 & 13 & 11 & 10 & 14 & 15 & 15 \\
\hline 34 & 13 & 10 & 11 & 11 & 11 & 16 \\
\hline 35 & 11 & 14 & 13 & 16 & 18 & 16 \\
\hline
\end{tabular}

Table 4.2: The MIP (in pe/MII) response for each of the 30 test beam tiles. The light yield was determined using the cosmic ray test stand.

\subsubsection{Calibration System}

A calibration system for the test beam ICD was also included [49]. A complete calibration system would have required transporting ultraviolet light to the tiles as was done for the DØ ICD tiles. Unfortunately, we discovered it was very difficult to transport UV light from the outside of the cryostat to the tiles on the inside. UV light can only be carried by quartz optical fibers, which crack at LAI temperatures. Therefore, it was decided to only calibrate the electronics. Light from a blue LED was fanned out to each of the PMT's. A six-level pulser was used to vary the light intensity. This system proved to be very useful in diagnosing and pinpointing problems with individual tiles and bases.

\subsection{Description of Test Beam Setup}

\subsubsection{General Setup}

Included in the load were four CCEM modules and two each of CCFH modules, CCCH modules, ECMH modules, and ECOH modules. The associated massless gaps, two CCMG's, two ECMH MG's and two ECOH MG's, were also included. Five $\phi$ regions were instrumented with the modified ICD design. Thus there were 10 boxes, with 30 tiles in all. Additionally, an ECMH and an ECOH massless gap were placed directly behind the ICD. This was originally planned and was left on in the design as a backup system to the scintillator. The 
arrangement of the load can be seen in the plan and elevation views in Figure 4.12 and Figure 4.13. The load spanned a $\phi$ range from 28 to 36 and an $\eta$ range from -0.5 to 1.4 . The ICD covered a $\phi$ range from 31 to 35 , with the usual $\eta$ range of 0.8 to 1.4 .

Originally, we planned to instrument four $\phi$ modules with the modified ICD design. Four $\phi$ regions means 24 individual tiles, and 24 sapphire glass interiaces for the 24 photomultiplier tubes. The test beam run plan committee later convinced us to include a fifth $\phi$. However, this decision was made after the feedthrough port which held the sapphire glass windows was designed. It proved impossible, due to space and monetary considerations, to add an extra six windows and tubes. Instead, we accommodated the extra six channels by ganging together six sets of output signals from two different tiles. This ganging was easily accomplished by bundling the fibers from the two tiles together into the same PVC collar. The ganging arrangement is shown in Table 4.3. Ganged tiles are indicated by matching symbols, unganged tiles are unmarked. The ganging scheme was based on maximizing the distance between the ganged tiles, where the distance was measured in $\left(\Delta \eta^{2} \times \Delta \phi^{2}\right)^{1 / 2}$. Additionally, we wanted to maintain a completely unganged $\phi$.

\begin{tabular}{|c||c|c|c|c|c|c|}
\hline$\phi \backslash \eta$ & 09 & 10 & 11 & 12 & 13 & 14 \\
\hline \hline 31 & $\diamond$ & $\triangle$ & $\square$ & & $\bigcirc$ & $\otimes$ \\
\hline 32 & & & & & & $\odot$ \\
\hline 33 & & & & & & \\
\hline 34 & & & & & & $\triangle$ \\
\hline 35 & $\bigcirc$ & $\odot$ & $\otimes$ & & $\diamond$ & $\square$ \\
\hline
\end{tabular}

Table 4.3: The output signal ganging scheme is shown. Ganged tiles are indicated by matching symbols.

The entire load was installed on a specially designed cart which held the modules at the appropriate angles of inclination. This cart was slid into the test beam cryostat, a double-walled cylindrical vessel 9 feet in diameter and 18.5 feet in length. There was a window along the long axis of the cryostat for the beam to enter with minimal scattering. The cryostat itself was mounted on a transporter platform which enailed the cryostat to 


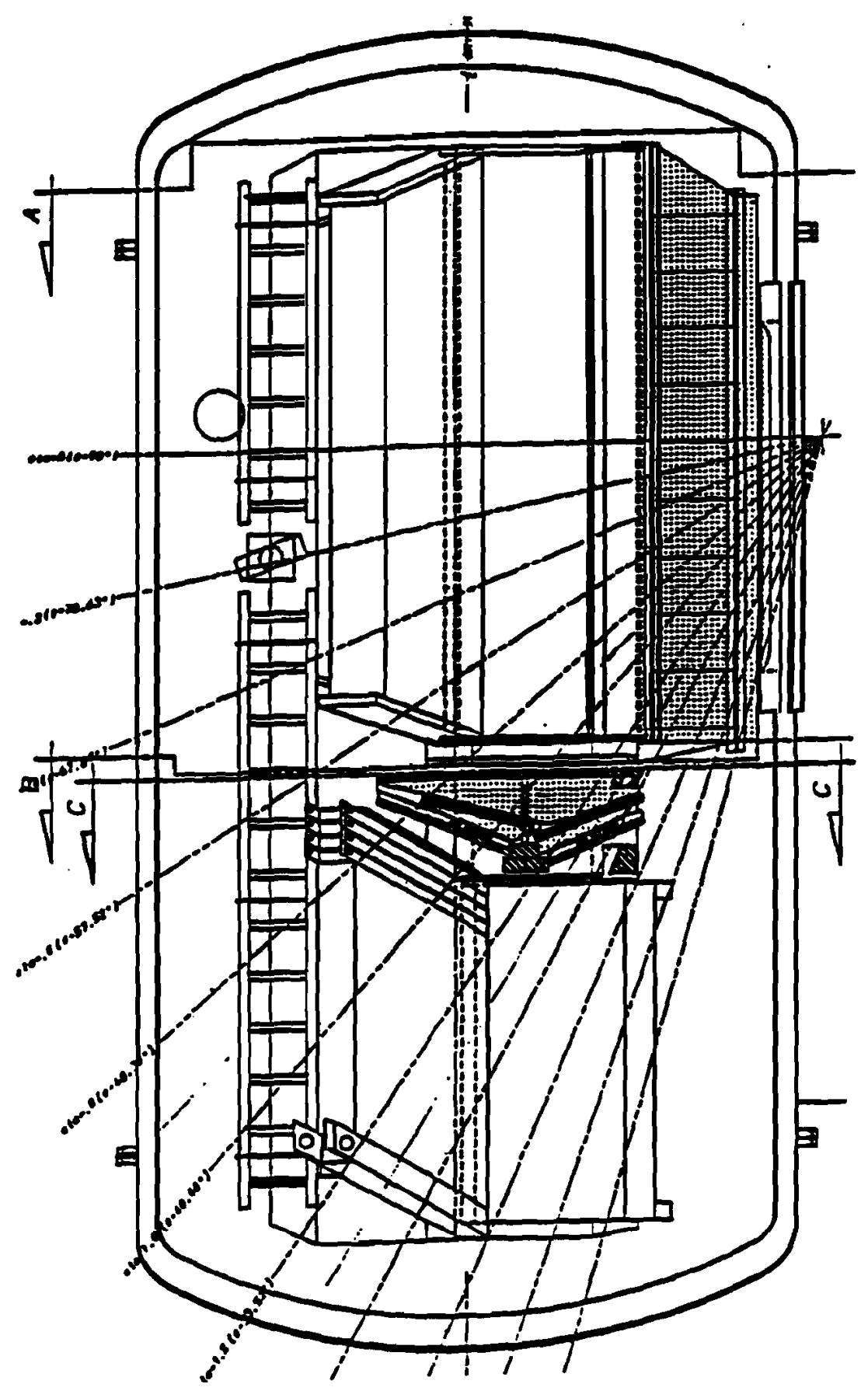

Figure 4.12: A plan view of the modules contained in the Load II run. The interaction point can be seen at the bottom of the picture. The central calorimeter modules are on the right. The end calorimeter modules are on the left. The Intercryostat Detector and Massless Gaps are highlighted in the center. The foam excluder is represented by the dotted area. 


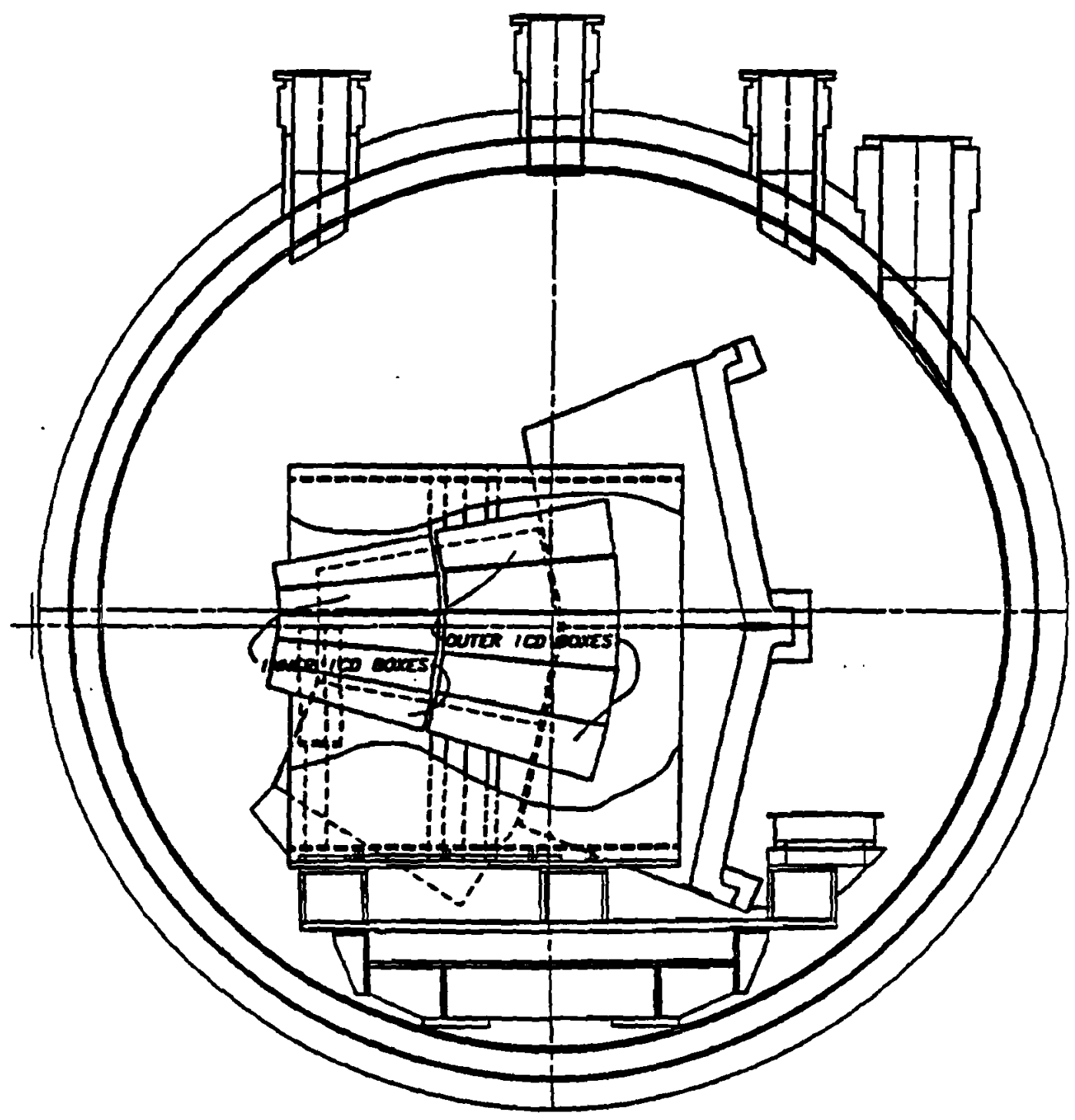

netron to

Figure 4.13: An elevation view of the modules contained in the Load II run. The outline of the cryostat and feedthrough ports can be seen. The ICD boxes are noted and the outline of the steel wall and end calorimeter modules can be seen behind the ICD boxes. 
have three degrees of freedom: rotation about both axes, plus lateral motion parallel to the length of the cryostat. With these three degrees of freedom, it was possible to closely reproduce the correct angles for an incident beam on a wide range of $\eta$ and $\phi$ positions of the modules inside the cryostat. The maximum $\phi$ position was $34.2^{\circ}$, which was just on the edge of the last row of ICD tiles.

\subsubsection{Intercryostat Region Setup}

A closer view of the intercryostat region simulation is shown in Figure 4.14. The double walls of the central cryostat were replaced with a single 1.0 inches thick steel wall [50]. The small protrusions at the high $\eta$ end of the wall simulated the extra material due to the corner of the CC cryostat. The end calorimeter cryostat was mocked up by a series of four steel pieces welded together. The cryostat walls were replaced by 1.25 inches steel plates, while the EC support ring, at $\sim \eta=1.1$, was replaced by thick steel pieces. It is important to note that in $D \emptyset$ the cryostat structures (obviously) follow the curvature of the cryostats. Thus, the CC corner and the EC support ring occur at the same $\eta$ for all $\phi$. However, in the test beam, it was impossible (too expensive) to reproduce the correct curvature of these structures. Thus, the mockup is best at one particular $\phi, \phi=32.5^{\circ}$.

The ICD modules were situated in the small gap between the dummy walls. There were several difficulties in the ICD placement. First, the dimensions of the steel walls were determined by trying to closely reproduce the absorption lengths of the actual walls and air gap $[50,51]$. This determination was done without including room for the ICD, as the ICD was not originally intended to be included in the load. Also, the actual cryostats are curved in $\eta$ space, whereas they were replaced by walls at a single angle in the simulation. The addition of the extra set of massless gaps which didn't exist in $D \emptyset$ (just in case the test beam ICD failed) used up a little bit more of that gap. All these effects combined to severely constrain the available space in which to place the ICD modules. As can be seen in Figure 4.14 , the low eta boxes were placed slightly forward of the high eta boxes. The edges of both boxes lie along the correct $\eta=1.1$ line. However, there are slight differences between the tile sizes and the other $\eta$ boundaries.

A foam excluder, Rohacell 71 supplied by Rohm Inc., was placed between the ICD modules and the walls to simulate the air gap [52]. 


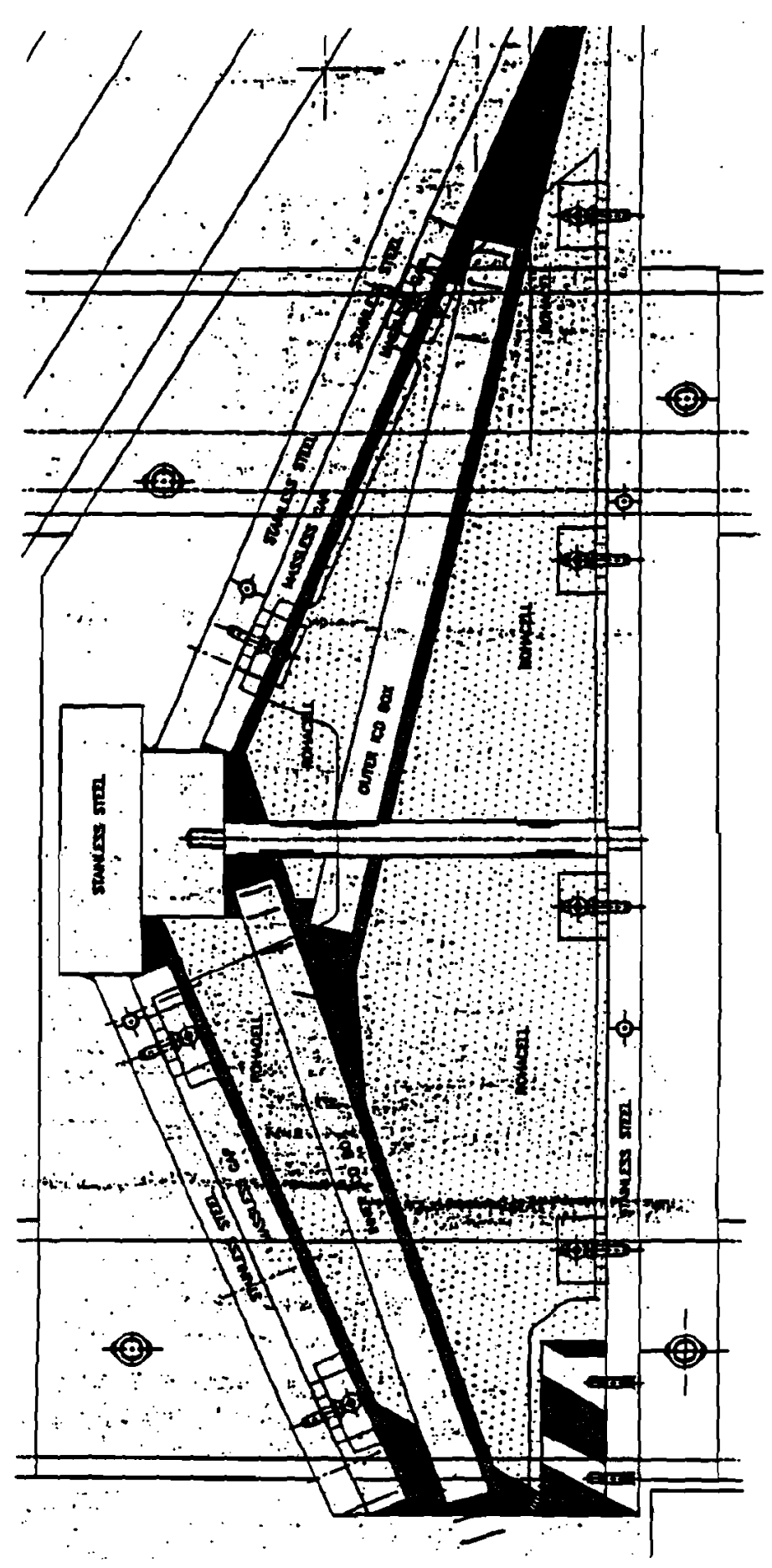

Figure 4.14: A close-up view of the intercryostat region of the test beam. 


\subsubsection{Beamline Elements}

The particle beam at NWA was created in several stages [53]. The NW beamline is shown in Figure 4.15. In the first stage, the $800 \mathrm{GeV}$ primary proton beam is extracted from the Tevatron steered onto an aluminum target, creating a secondary beam of particles. A downstream sweeper magnet, NW4S, was available to clean this beam of unwanted charged particles. The secondary beam was then focussed, steered and defined with a series of magnets, collimators and lead target wheels. The secondary beam went through five enclosures, NW4, NW6, NW7, NW8, and NW9, before reaching the cryostat in NWA. A series of magnets in NW4, NW6 and NW7 produced three horizontal bends. NW4W and NW7W produced the west bends and NW6E produced the east bends. Several other magnets were used to maintain the vertical focus of the beam (NW4V, NW6V, NW7V and NW9V).

There were three lead target wheels, NW4PB, NW6PB and NW7PB, which were used to create the different beam types. Electrons were created by first sweeping the charged particles out of the beam with NW4S. A lead plate at NW4PB then converted the photons in the beam into electrons. Pions were created by inserting thin lead plates at NW6PB and NW7PB which scattered any electrons out of the beam. A series of horizontal and vertical collimators ( $N W 4 \mathrm{CH}, \mathrm{NW} 4 \mathrm{CV}, \mathrm{NW6CH}$ and $\mathrm{NW6CV}$ ) were also used to optimize the beam size.

A series of three proportional wire chambers was used in the beamline to determine the momentum of the incoming beam particles. NW8PWC and NW9PWCB provided the measurements in the $x$-plane and NW9PWCA provided the $y$-plane information. A fourth PWC, NWAPWC, was mounted on the cryostat to provide a final position determination of the beam. Various scintillator paddles were placed in the beamline to provide beam intensity information. A set of three scintillators in NWA were used as the event trigger. Scintillator paddles were installed downstream (behind) the cryostat to provide either a veto for muons in the pion beam or to provide the trigger for the muon beam. Two Cerenkov counters were used to provide particle identification and electron tagging. 


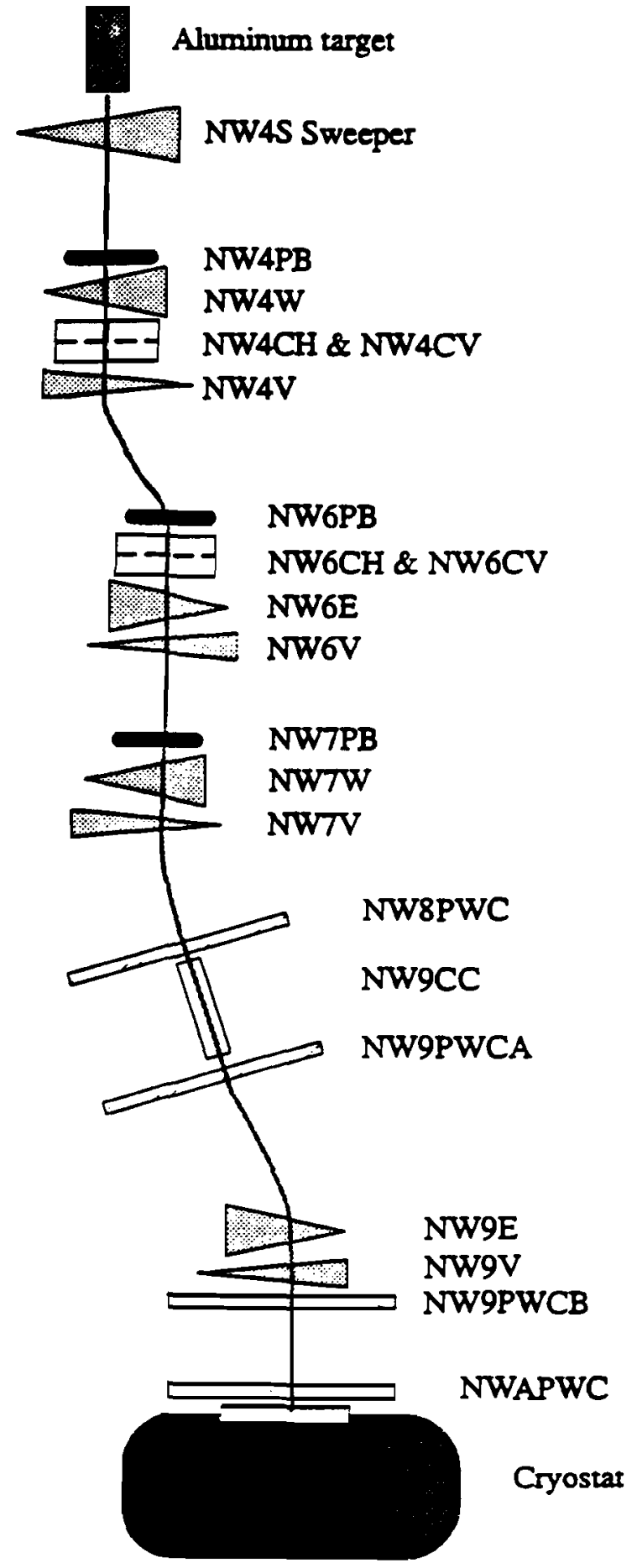

Figure 4.15: The Neutrino West beamline. The beamiline elements are discussed in the text. 
CHAPTER 5

DATA ANALYSIS

\subsection{The ICD Test Beam Mini-DST Package}

There were over 5000 channels of calorimetry in operation at the test beam. Even with suppression of inactive channels, the size of a data file from a single run quickly became very large. In order to decrease the disk space requirements and to facilitate analysis in general, we developed a mini-DST package. ("Data Summary Tapes").

The DST package utilizes a user-defined road around the beam to save relevant calorimeter information. The road is defined such that information from all calorimeter cells satisfying the criteria:

$$
\begin{aligned}
& \left|\eta_{\text {cell }}-\eta_{\text {beam }}\right|<\Delta \eta \\
& \left|\phi_{\text {cell }}-\phi_{\text {beam }}\right|<\Delta \phi
\end{aligned}
$$

is saved. $\Delta \eta$ and $\Delta \phi$, the road size, are defined based on particle type, but can also be easily changed by the user. In addition to saving the above calorimeter cells, all information from each of the detectors in the intercryostat region (ICR) is saved. This includes the Intercryostat Detector, the $C C$ massless gaps and the EC massless gaps. In addition, information from the survey, transporter, CAMAC and PWC's is kept for each event. The DST has a data reduction factor of approximately 30 .

The DST package is based on a standard DØ offline analysis package, CALOR_OFF (for CALORimeter OFFline analysis). It takes advantage of many of the useful CALOR_OFF packages already developed. Information from the test beam survey, camac and the PWC's 
are each accessed via a single call to a pre-existing CALOR_OFF package. Individual channel gain corrections are also incorporated. The complete DST package has been described in an internal $\mathrm{D} \emptyset$ Note [54].

\subsection{Discussion of Energy Loss in Matter}

A brief discussion of the energy loss of particles as they pass through matter would be helpful at this point. As a charged particle goes through matter, it loses energy through several processes. These processes include ionization of the electrons in the target medium, nuclear recoil energy loss, and radiative effects such as bremsstrahlung and pair production. At moderately relativistic velocities, energy loss through ionization dominates and is given by the Bethe-Block equation:

$$
-\frac{d E}{d x}=4 \pi N_{A} r_{e}^{2} m_{e} c^{2} z^{2} \frac{Z}{A} \frac{1}{\beta^{2}}\left[\ln \left(\frac{2 m_{e} c^{2} \gamma^{2} \beta^{2}}{I}\right)-\beta^{2}-\frac{\delta}{2}\right]
$$

where $z e$ is the charge of the incident particle; $\beta c$ is the velocity of the incident particle; $Z$ and $A$ are respectively the atomic number and weight of the target medium; $m_{e}$ and $r_{e}$ are respectively the mass and classical radius of the electron; $I$ is the ionization constant and is given by $16 Z^{0.9} \mathrm{eV}$ for $Z>1$; and $\delta$ is a constant which represents the decreased energy loss due to shielding effects from the charge density of the target nuclei.

The mean energy loss is given by

$$
\Delta E=\frac{d E}{d x} \delta x
$$

where $d x$ is measured in mass per unit area $\left(\mathrm{g} \mathrm{cm}^{-2}\right)$ and $\delta x$ is the thickness of the material through which a particle passes. Generally there will be large fluctuations about this mean. If the mean energy loss in a given thickness is greater than the maximum energy transferred in a single collision (i.e., $\delta x$ is very large) then the fluctuations will have a Gaussian distribution. However, for most physical situations, $\delta x$ will be small and the fluctuations will have a long tail. These fluctuations are well described by a Landau distribution [55]:

$$
\mathcal{L}(\boldsymbol{x})=\operatorname{Aexp}\left(\frac{-1}{2}\left(\beta+e^{-\beta}\right)\right)
$$

with

$$
\beta=\left(x-x_{0}\right) /\left(x_{0} b\right)
$$


where $\boldsymbol{x}_{0}$ is the location of the distribution peak or the most probable value, $A$ is a constant related to the height of the distribution, and $b$ is a factor related to the width of the distribution.

\subsection{Muon Calibration of Test Beam Tiles}

As mentioned above, scintillator based detectors, such as the ICD, have inherent differences in response between detector elements, in this case, the individual tiles. For the D $\emptyset$ ICD tiles, individual calibration of each tile's response to minimum ionizing radiation was performed using cosmic rays. Likewise, each of the test beam tiles was tested with cosmic rays for an in-air calibration. However, in the test beam cryostat, it was not possible to use cosmic rays to measure the MIP response in LAr. Instead, tile calibration was done using a beam of muons as the minimum ionizing particles.

Two full muon scans were done, aimed at each of the 30 test beam tiles. The first scan was taken early in the run (August) using $50 \mathrm{GeV} \mu$ 's. The second scan was completed late in the run (November and December) using $150 \mathrm{GeV}$ and $100 \mathrm{GeV} \mu$ 's. In addition, some $15 \mathrm{GeV}$ and $50 \mathrm{GeV}$ runs were taken at the later time. The two full sets of scans allow us to perform comparison studies with this data, in addition to providing the critical MIP calibration information.

Typical distributions of ADC counts from a muon beam going through a tile are shown in Figure 5.1. The distributions shown are the response from the tile at which the beam was aimed. Important features to note are the well-defined peak and long tail. Some of the tiles also have a large peak near zero. This peak is due either to pedestals or to effects such as a mistuned or poorly aimed beam, a large beam profile which spanned several tiles, or, in the case of Figure 5.1(b), transporter limitation problem. In the above cases, the major energy deposition will usually be in a cell adjoining the tile where the beam was nominally aimed. For analysis purposes, a cut was made to remove those events in which the maximum energy deposition was not in the beam tile. In addition, if any peak remained at zero after the previous cut, that peak was removed from the fit (as the fitting routine only uses non-zero channels, those channels were easily removed by zeroing those channels in the histogram). For some runs, removing those channels severely degraded the fit. These runs were were not used in the following analyses.

In order to extract the MIP normalization from the muon data, several fitting functions 

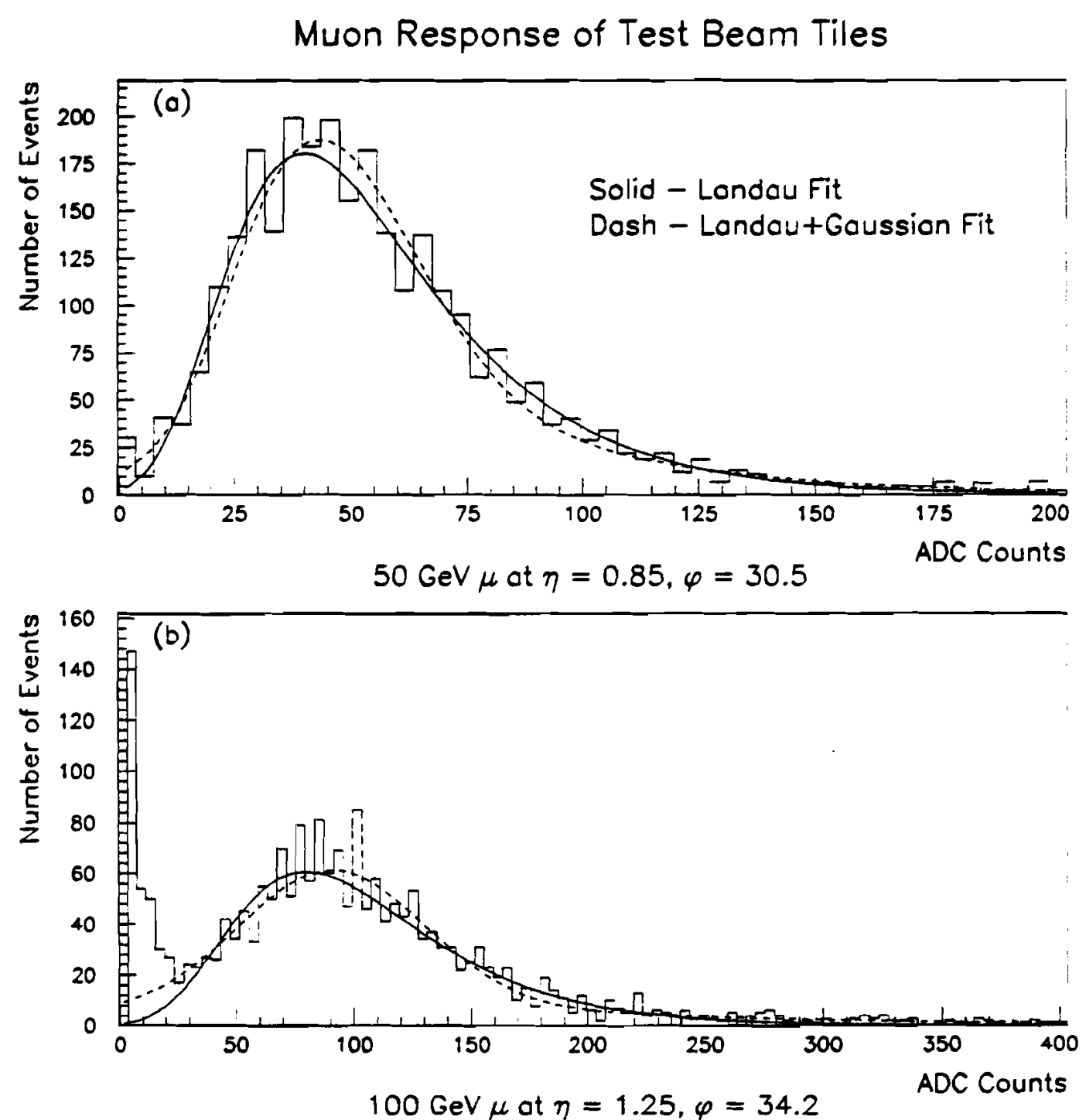

Figure 5.1: Typical distributions of ADC counts for a muon going through a test beam ICD tile. 
were examined. The standard fitting package, MINUIT [56], was employed to determine the fits. The first function studied was a simple Gaussian distribution, which was used to fit the peak only. The long tails and any initial peak were removed from the fit. The main purpose behind using the Gaussian was to get a first order approximation of the MIP normalization which was needed in order to determine the first order ICD sampling fractions urgently needed by $D \emptyset$ before the beginning of the collider run. The Gaussian values agreed (on the order of $10 \%-15 \%$ ) with the other fitting functions studied.

Two other fits to the muon peaks were studied before the final determination of the MIP normalization constants. The first was a Landau distribution:

$$
\mathcal{L}=a_{1} \exp \left(-a_{2}\left(\beta+e^{-\beta}\right)\right)
$$

with

$$
\beta=\left(x-x_{0}\right) /\left(x_{0} a_{3}\right)
$$

where $\mathbf{x}$ is the number of adc counts, $a_{1}$ is a constant related to the height of the distribution, $x_{0}$ is the location of the distribution peak and $a_{2}$ and $a_{3}$ are factors related to the width of the distribution. Nominally, $a_{2}=1 / 2$, but much better fits could be obtained by allowing both $a_{2}$ and $a_{3}$ to vary. The double exponential of the Landau function takes into account the long tail of the MIP distribution very well.

The second function studied was a sum of a Landau distribution plus a Gaussian distribution:

$$
\mathcal{L G}=a_{1} \exp \left(-a_{2}\left(\beta+e^{-\beta}\right)\right)+\exp \left(-1 / 2\left(x-x_{0}\right) /\left(a_{4}\right)\right)
$$

with

$$
\beta=\left(x-x_{0}\right) /\left(x_{0} a_{3}\right)
$$

where the parameters, $a_{n}$ are the same, with the addition of $a_{4}$, the width of the Gaussian distribution. Systematic noise due to experimental apparatus is usually well described by a Gaussian distribution. Thus, including a Gaussian term often improves the overall fit. However, in this case, adding the Gaussian lowers the overall $\chi^{2}$ of the fit very slightly. This is because the intrinsic noise in a scintillator detector is small.

In Figure 5.1, the Landau and Landau plus Gaussian fits are superimposed over the distributions. As one can see, both fits do an excellent job of fitting the muon signal. For comparison, the peak values and $\chi^{2}$ values for these two tiles are listed in Table 5.1. 


\begin{tabular}{|l|c|c|}
\hline$\eta=0.85, \phi=30.5$ & Peak Value & $\chi^{2}$ \\
\hline Landau fit & 39.90 & 2.120 \\
\hline Landau + Gaussian fit & 43.32 & 2.109 \\
\hline$\eta=1.25, \phi=34.2$ & Peak Value & $\chi^{2}$ \\
\hline Landau fit & 80.05 & 1.706 \\
\hline Landau + Gaussian fit & 90.96 & 1.241 \\
\hline
\end{tabular}

Table 5.1: Comparison of Landau and Landau + Gaussian fits to two sample muon distributions.

The $\mathcal{L}$ distribution tends to rely on the leading edge of the peak while the $\mathcal{L} \mathcal{G}$ distribution tends to fit the tails better. This results in a slightly higher peak value for the $\mathcal{L G}$ fit. A comparison of the peak values for all tiles, as determined by each type of fit, is shown in Figure 5.2. Overall, the peak value as dettrmined by the $\mathcal{L}$ fit is $8.8 \%$ lower than the peak value as determined by the $\mathcal{L G}$ fit.

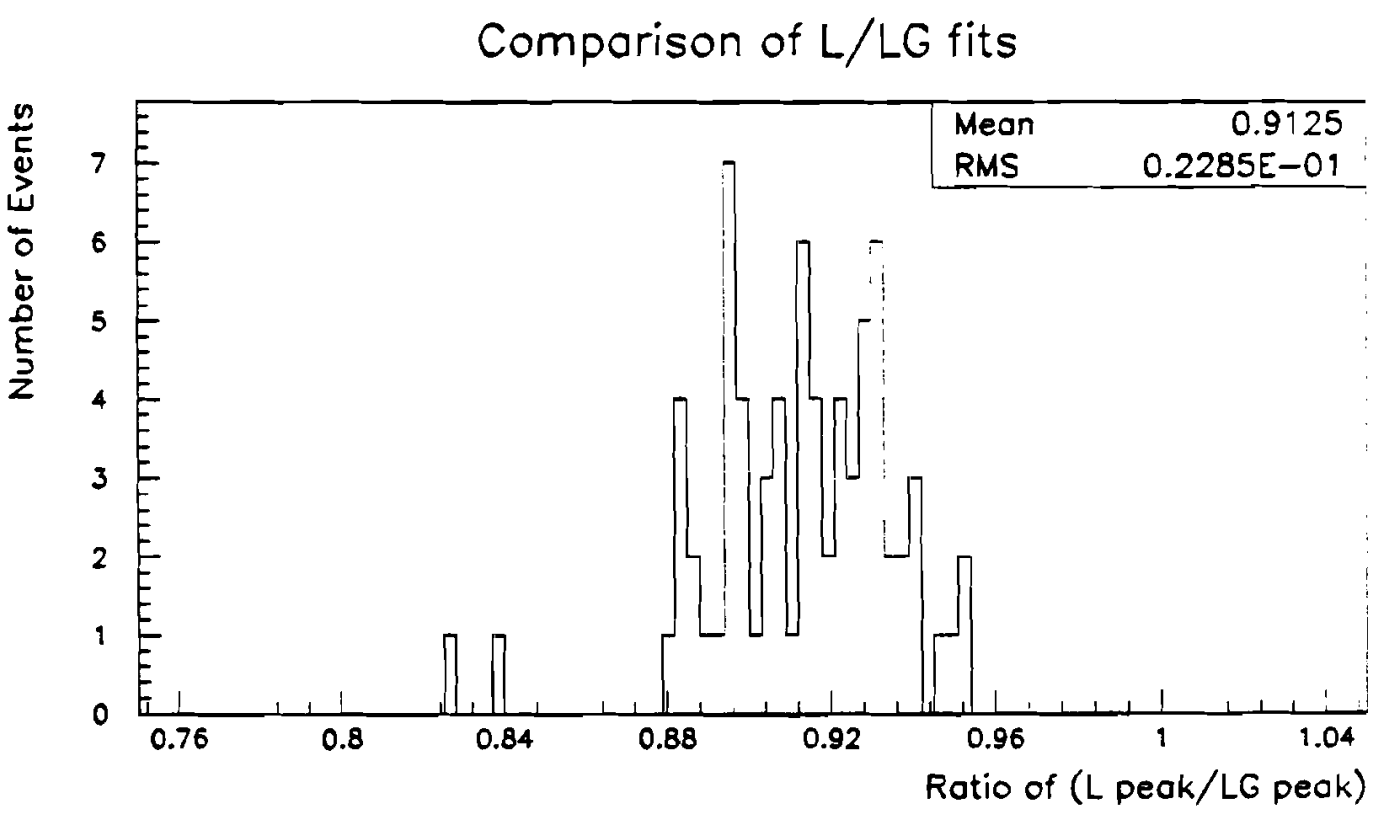

Figure 5.2: The ratio of peak value as determined by a Landau fit to the peak value as determined by a Landau + Gaussian fit.

In the $D \emptyset I C D$, a Landau distribution was used to fit the cosmic ray data in order to determine the MIP normalization for each of the 768 tiles. Given that and the overall 
negligible $\chi^{2}$ improvement in the LG distribution, it was decided to use peak values as determined by the Landau distribution for the test beam tiles also. Most importantly, by using the same MIP normalization for each set of tiles, test beam ICD and real ICD, we could directly transfer the calibration constants from the test beam to $D \emptyset$. The values for the MIP normalizations of the 30 test beam tiles are shown in Table 5.2. The three dead tiles were defined to have a MIP value of 999.9 .

\begin{tabular}{|c||c|c|c|c|c|c|}
\hline$\phi \backslash \eta$ & 09 & 10 & 11 & 12 & 13 & 14 \\
\hline \hline 31 & 36.09 & 18.02 & 999.9 & 52.00 & 41.50 & 205.0 \\
\hline 32 & 28.80 & 27.28 & 48.30 & 78.00 & 49.00 & 133.0 \\
\hline 33 & 32.95 & 26.50 & 21.94 & 29.75 & 38.75 & 37.50 \\
\hline 34 & 37.50 & 23.75 & 33.25 & 56.00 & 83.96 & 31.00 \\
\hline 35 & 26.75 & 28.50 & 999.9 & 46.95 & 90.50 & 999.9 \\
\hline
\end{tabular}

Table 5.2: MIP Normalization constants for the 30 test beam tiles.

The rather large variation in the MIP constants between tiles was because all the tiles were set to the same operating voltage. The optimum procedure would have been to take a muon scan of all the tiles, adjust the individual PMT operating voltages so that all tiles had a MIP normalization constant in a defined dynamic range, and then take a new scan immediately following. However, a muon scan of all 30 tiles took an appreciable amount of time and there was insufficient time in the test beam schedule to allow this optimum calibration of the tiles. The minimal dynamic range needed at the test beam meant a voltage plateau was not critical to the MIP calibration results.

\subsection{Results from Studies with Muon Beams}

One of the advantages to having two (nearly) complete scans of the test beam tiles is that it allows us to study long-term effects such as detector stability in LAr and stability of the MIP calibration. However, the muon scans were done at different energies. Therefore, before we can use the scans to look at the stability of the detector over time, we need to understand the energy dependence of the detector. 


\subsubsection{Energy Dependence of MIP Calibration}

A high-energy muon is a good, but not perfect, approximation to a minimum ionization particle. In general, there is a slight energy dependence of the muon signal in a sampling detector. At higher muon energies, energy loss from pair production, bremsstrahlung and nuclear interactions plays a greater role in the total muon energy loss, eventually becoming greater than the loss through ionization. Thus the muon is no longer a minimum ionizing particle and the detector response to muons will slowly increase with energy. This energy dependence is greater in a multi-layered detector since the radiated energy is contained and sampled and adds to the MIP peak in each layer. A previous test beam study showed a $\sim 4 \%$ rise in the response of the ECEM to muons over the energy range $15 \mathrm{GeV}$ to $150 \mathrm{GeV}$ [57]. The end calorimeter has four readout layers and 21 layers of cells consisting of argon gaps and uranium absorbers. The ICD consists of only a single layer of sampling plus steel absorbers (cryostat walls). Thus, we did not expect to see much energy dependence for the ICD.

The first complete muon scan of the tiles was done at $50 \mathrm{GeV}$, while the latter scan was done mainly at $150 \mathrm{GeV}$, with a few $50 \mathrm{GeV}$ and $100 \mathrm{GeV}$ runs. In addition, two tiles had runs taken at $15 \mathrm{GeV}$. In order to determine if there is any systematic dependence on the energy of the muon, we can compare the peak tile response in a particular tile at two different energies. Figure 5.3 shows the fractional difference in the peak tile response, where the fractional difference is given by:

$$
R=\frac{x_{2}-x_{1}}{x_{2}}
$$

where $x_{2}$ and $x_{1}$ are the peak values for two different scans in the same tile. In each case, $x_{2}$ was the peak of the tile response for the higher energy run; $x_{1}$ the peak for the lower energy run. In Figure 5.3, graph (a) shows the distribution of the fractional difference for all energy comparisons possible, graph (b) is the distribution which compares the response of $150 \mathrm{GeV}$ muons to $50 \mathrm{GeV}$ muons; graph (c) compares the response of $100 \mathrm{GeV}$ muons to $50 \mathrm{GeV}$ muons; and graph (d) compares the response of $50 \mathrm{GeV}$ muons to $15 \mathrm{GeV}$ muons. For additional information, Figure 5.4 plots the fractional difference as a function of time between runs. The energies being compared are noted.

For all energy comparisons, the average of the fractional difference is -0.0097 , with an rms error of 0.0781 . The averages and rms errors in the fractional difference for the various 
Comparison of response for different energies
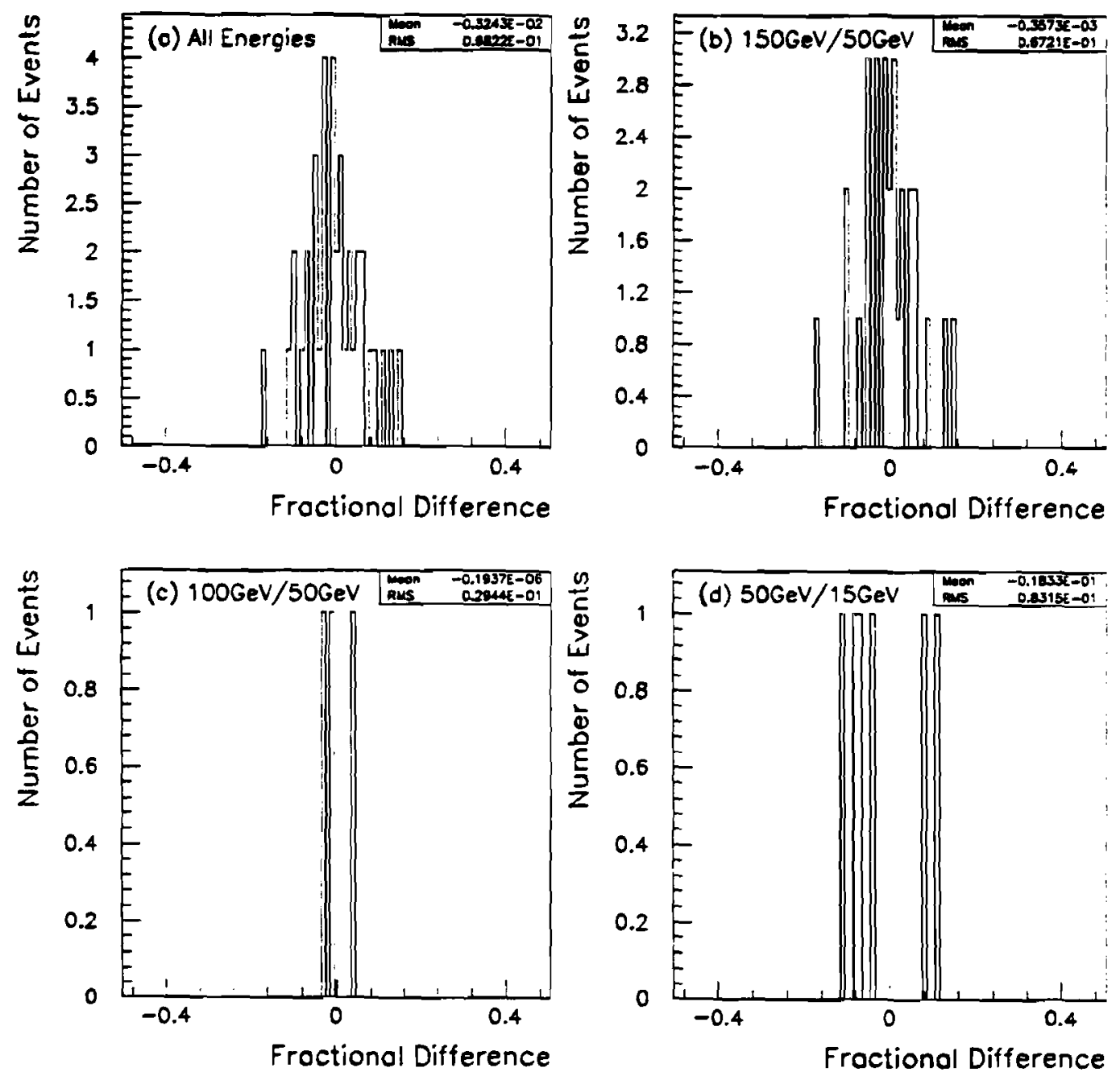

Figure 5.3: The fractional difference in tile response for various tiles to different muon energies. The distribution of the fractional difference is shown for the several different energies being compared. 


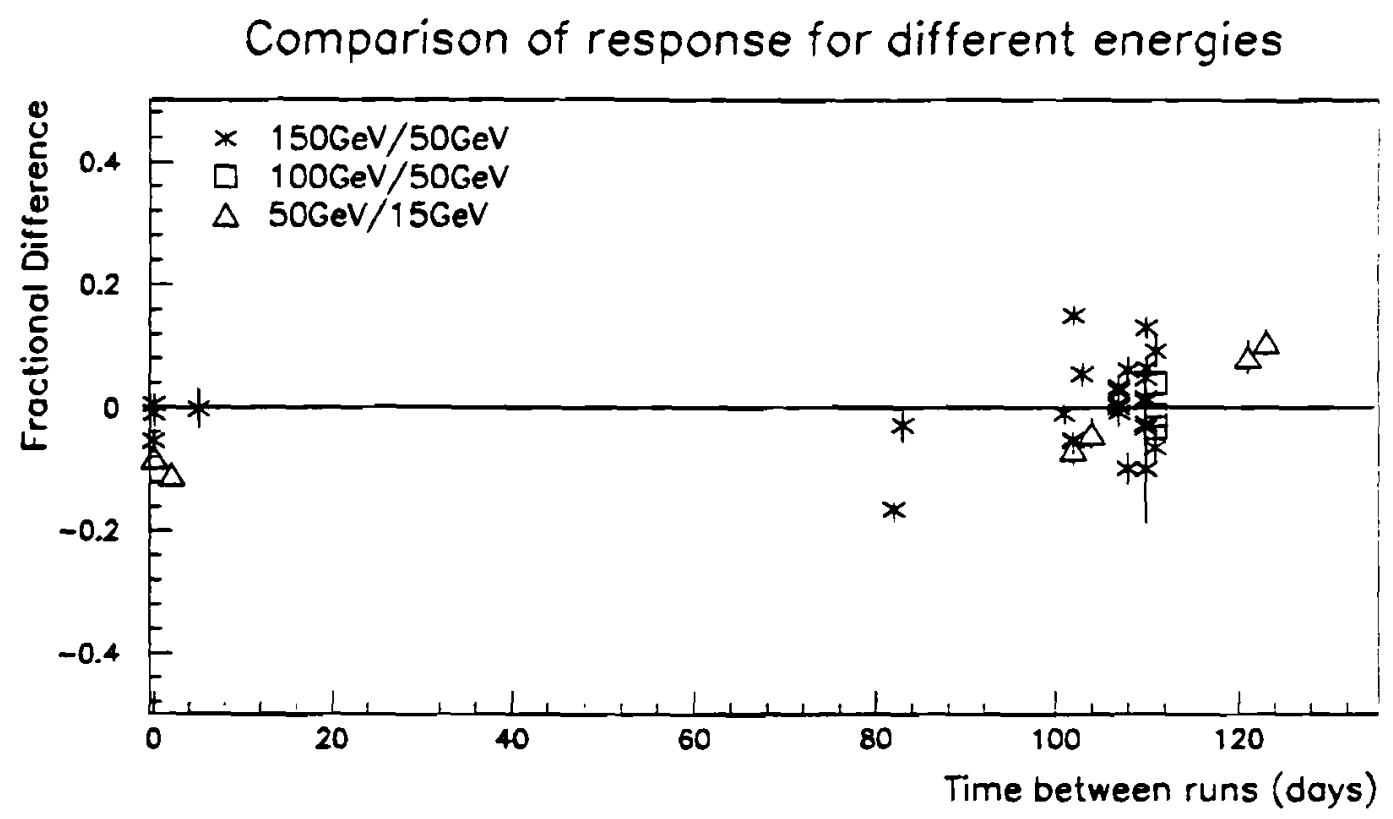

Figure 5.4: The fractional difference in tile response to different muon energies is plotted as a function of time between runs.

energy comparisons are summarized in Table 5.3. For comparison purposes, the table also includes the average fractional difference and rms error for tiles which had more than one muon run at the same energy at different times. Figure 5.5(a) shows the distribution of the fractional difference for same energy runs and $5.5(\mathrm{~b})$ plots the fractional difference as a function of the time between the runs.

For all the energy comparisons, the fractional difference is slightly negative. This negative shift is in the opposite direction expected if there is a true energy dependence in the tile response. Within the rms error of each the distributions, the shift is compatible with zero. Therefore, we conclude there is no discernible increase in response due to the energy of the muon and we can directly use the two sets of muons scans to study the long-term stability of the detector response.

\subsubsection{Stability of Detector Response}

In order to look at the stability of tile response over the length of the test beam run, we consider Figure 5.6, which plots the fractional difference in tile response as a function of 


\begin{tabular}{|c|c|c|c|}
\hline $\mathrm{E}_{1}(\mathrm{GeV})$ & $\mathrm{E}_{2}(\mathrm{GeV})$ & <Fractional Difference & RMS error \\
\hline 50 & 150 & $-0.357 \mathrm{E}-03$ & $0.672 \mathrm{E}-01$ \\
\hline 50 & 100 & $-0.194 \mathrm{E}-06$ & $0.294 \mathrm{E}-01$ \\
\hline 15 & 50 & $-0.183 \mathrm{E}-01$ & $0.832 \mathrm{E}-01$ \\
\hline low & high & $-0.974 \mathrm{E}-02$ & $0.781 \mathrm{E}-01$ \\
\hline same & same & $-0.144 \mathrm{E}-01$ & $0.385 \mathrm{E}-01$ \\
\hline
\end{tabular}

Table 5.3: A comparison of the peak tile response to muons of different energies. The average of the fractional difference in response between the two noted energies is given, along with the rms error of the average.

Comparison of response for same energies
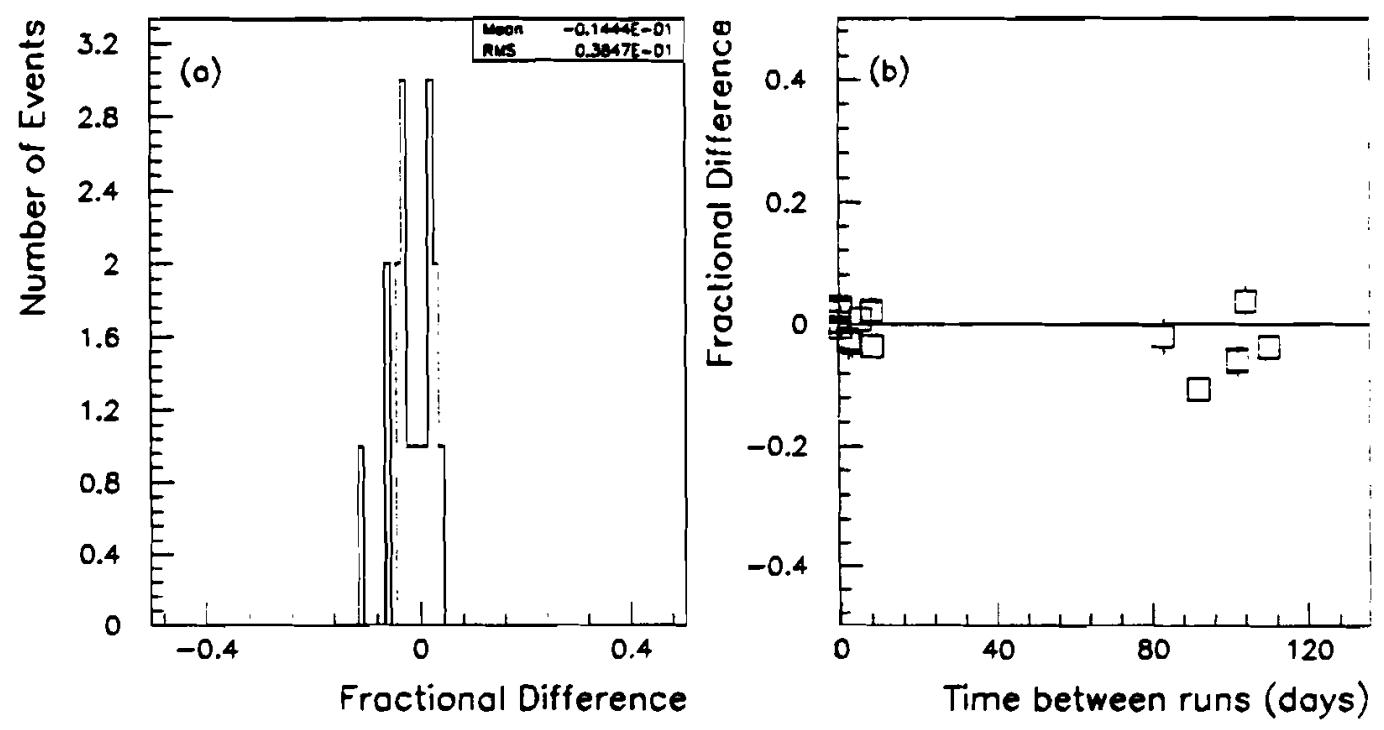

Figure 5.5: The fractional difference in tile response for various tiles to the same muon energy. The distribution of the fractional difference is shown, along with plotting the fractional difference as a function of time between runs. 

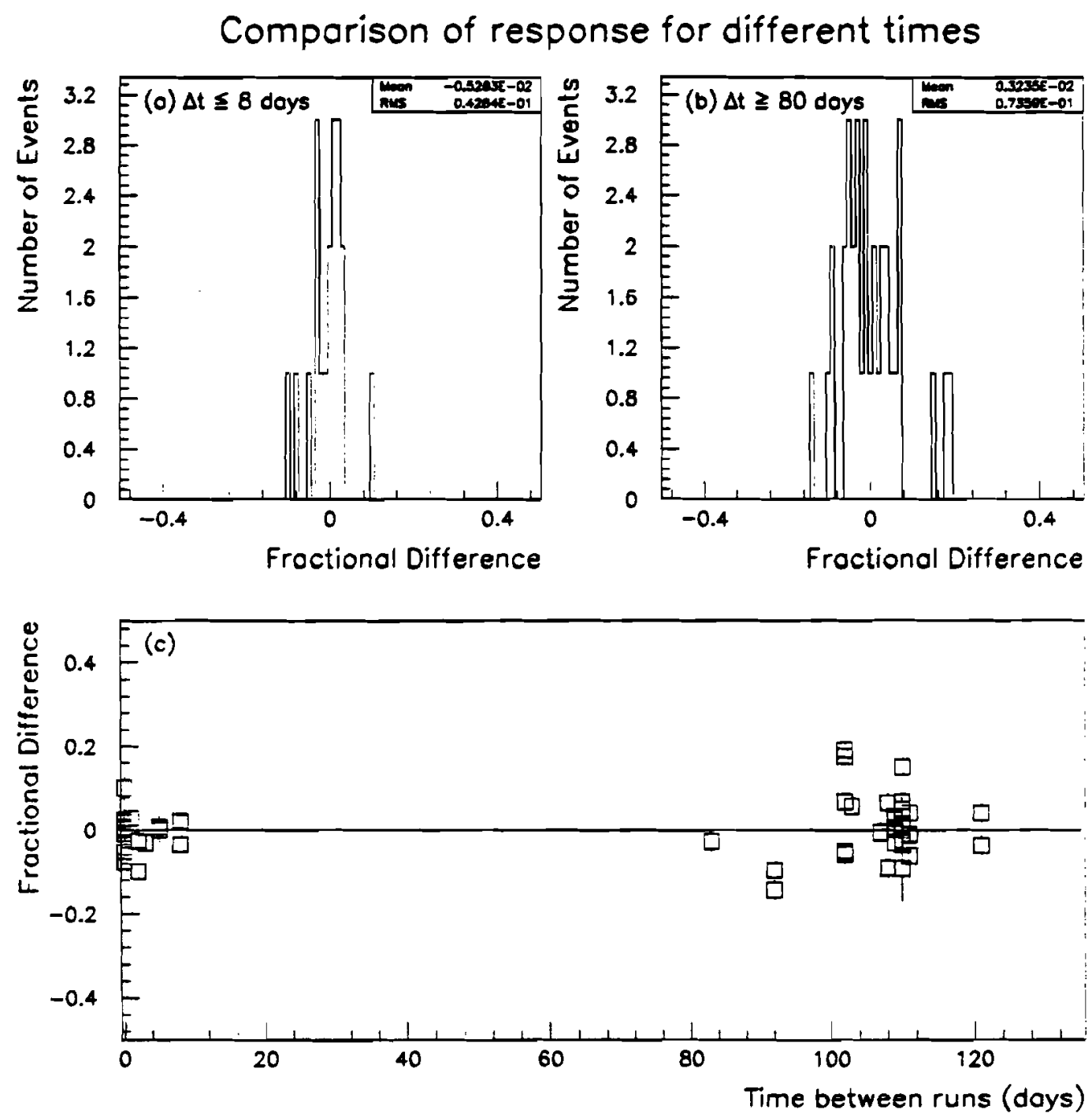

Figure 5.6: The fractional difference in tile response for all the test beam tiles as a function of time between two runs. 
time between runs. Similarly, the fractional difference is given by:

$$
R=\frac{x_{2}-x_{1}}{x_{1}}
$$

where $x_{2}$ and $x_{1}$ are the peak values for two different scans in the same tile. In each case, $x_{2}$ was the peak of the tile response for the later (in time) run; $x_{1}$ the peak for the earlier run. All muon energies are used in this analysis. Graph 5.6(a) shows the distribution of the fractional difference in response for runs taken a very short time apart ( $\Delta t \leq 8$ days) and graph $5.6(\mathrm{~b})$ is the distribution for runs taken a long time apart ( $\Delta t \geq 80$ days). Graph 5.6(c) plots the fractional difference in tile response as a function of time between runs. In both (a) and (b), the mean of the distribution is compatible with zero. The Ims spread of the distribution is a measure of how accurately we can determine the peak of the muon response. The distribution in graph (b) has an rms spread of $7.4 \%$. We therefore assign a systematic error of $7.4 \%$ in the determination of the MIP normalization constants and will be carrying that error over into further analyses.

\subsection{Determination of Sampling Fractions}

\subsubsection{Introduction}

In a sampling detector, a particle loses energy as it traverses both the active medium and the inactive medium. The energy lost in the active medium is measured through the ionization signal of the medium. However, one would really like to know the total energy lost as the particle passed through the detector. This total energy can be found by relating the energy in the dead material to the live energy via the sampling fraction:

$$
s f=\frac{E_{\text {live }}}{E_{\text {dead }}+E_{\text {live }}} .
$$

$E_{\text {live }}$ and $E_{\text {dead }}$ can be calculated using known $d E / d x$ energy losses through matter. For instance, a single CCFH LAr/U cell consists of two argon gaps, a uranium absorber plate, a piece of G10 signal board, and a thin copper pad on the signal board. The thickness, density and mean $d E / d x$ loss for each of these materials is listed in Table 5.4. Also calculated is $\Delta E$ $=\mathrm{N} \delta x \rho d E / d x$, the total energy loss in the medium. The total live energy is $0.929 \mathrm{MeV}$, the total dead energy is $12.843 \mathrm{MeV}$, for a sampling fraction of $6.75 \%$. 


\begin{tabular}{|c|c|c|c|c|c|c|}
\hline Material & $\delta \times(\mathrm{cm})$ & $\mathrm{N}\left(\frac{\#}{\mathrm{cell}}\right)$ & $\rho\left(\frac{\mathrm{g}}{\mathrm{cm}^{3}}\right)$ & $\mathrm{dE} / \mathrm{d} \times\left(\frac{\mathrm{MeV}}{\mathrm{B} / \mathrm{cm}^{2}}\right)$ & $\Delta \mathrm{E}(\mathrm{MeV})$ & Type \\
\hline LAr gap & 0.2198 & 2 & 1.40 & 1.51 & 0.929 & live \\
\hline Ur plate & 0.5994 & 1 & 18.95 & 1.09 & 12.381 & dead \\
\hline G10 board & 0.1144 & 1 & 1.70 & 1.87 & 0.364 & dead \\
\hline Cu pad & 0.0076 & 1 & 8.96 & 1.44 & 0.098 & dead \\
\hline
\end{tabular}

Table 5.4: An example of how to calculate a sampling fraction. The sampling fraction for a single CCFH cell is calculated using the thicknesses, densities, and $d E / d x$ energy losses for the materials which make up the cell.

In the intercryostat region (ICR), calculation of the sampling fractions is not quite as simple. The amount of dead material before and after each of the three ICR detectors (ICD, CCMG and ECMG) is a rapidly changing function of $\eta$. Rather than calculating nominal $d E / d x$ sampling fractions, we decided to instead determine them by using the test beam data. In the following analysis, the general idea is to optimize the sampling fractions for the three detectors in the intercryostat region, CCMG, ICD, and ECMG, such that the total energy deposited in the detector is equal to the total input energy of the beam. For various reasons, in the $D \emptyset$ software system, the calorimeter sampling fractions were all normalized to the response of the end calorimeter electromagnetic layer 3 , i.e., the sampling fraction of ECEM3 was defined to be 1.000 and all other sampling fractions were divided by the ECEM3 response. Thus we are not calculating true sampling fractions, but rather 'sampling weights'.

\subsection{2 $\chi^{2}$ Optimization Technique}

The method is a standard technique in which $\chi^{2}$ is minimized with respect to the sampling weights, $s=s(\eta$, layer $) . \chi^{2}$ is calculated as the difference between the nominal beam energy, $E_{0}$, and the optimized energy seen in the calorimeter:

$$
\chi^{2}=\sum_{j}\left[E_{0}-\sum_{\text {cells }} E_{j}\right]^{2} .
$$

$E_{j}$ is the energy in a cell for the $j t h$ event and is given by:

$$
E_{j}(\eta, \phi, \text { layer })=A(\text { detector }) * s(\eta, \text { layer }) * C(\eta, \phi, \text { layer }) * Q(\eta, \phi, \text { layer })
$$


$Q(\eta, \phi$, layer $)$ is the charge in that cell measured in test beam ADC counts, gain corrected and pedestal subtracted. $A$ (detector) is the $A D C-t o-G e V$ conversion factor and varies slightly for the different subdetectors. The sampling weights, $s$ ( $\eta$, layer) are a function of $\eta$ only for the intercryostat region detectors. The constants, $C(\eta, \phi$, layer $)$, are used to correct for small detector dependent effects such as differences in absorber plate thicknesses. In the case of the ICD, they are the MIP normalization constants.

For this analysis, the fitting package MINUIT was utilized and the following constraints were placed on the data:

- $100 \mathrm{GeV} \pi$ data was used.

- A 17 I $17(\Delta \eta \times \Delta \phi)$ road around the beam was used (only those channels within the road were saved and used).

- Non-zero suppressed data was used.

- $\mu$ 's, e's and poorly reconstructed tracks, as defined by the beamline counters, were discarded.

- Leakage events, in which the shower started in the coarse hadronic layers, were discarded.

- The sampling weights for the electromagnetic layers were fixed.

- The ADC-to- GeV constants were fixed for the electromagnetic layers.

- The ADC-to- GeV constants for the hadronic layers were fixed.

A large road was chosen to be sure to get all the energy in the event. We chose to use non-zero suppressed data as zero suppression was known to have a few percent effect on the total measured energy. The sampling weights and ADC-to- $\mathrm{GeV}$ constants were fixed to values determined by previous analyses. The sampling weights for the calorimeter layers were fixed to the $d E / d x$ values [58]. The ADC-to-GeV constants were determined from test beam data (Load II and Load II) by optimizing the response of the various calorimeters to the total beam energy (e.g., CCEM, ECEM, hadronic layers) $[59,60,61]$. In addition, for the ganged tiles, all the deposited energy was assigned to one of the two ganged tiles based on the distance of closest approach to the actual beam tile. 
Determining the sampling weights was a very iterative process. Each data run was taken at a single $\eta$ within the intercryostat region $(0.7<\eta<1.4)$. However, a pion shower is very broad and spans over many $\eta$ and $\phi$. An incorrect sampling weight for a neighboring layer or $\eta$ can severely impact the optimization at the $\eta$ in question. Therefore, one can't simply determine the sampling weight at the beam $\eta$, independent of the other sampling weights in the intercryostat region. In order to get a zeroth order approximation to the sampling weights, we first assumed a single, constant weight for each of the three intercryostat layers (CCMG, ICD, and ECMG). We determined that single layer constant at each of the relevant $\eta$ positions: $\eta=0.75,0.85,0.95,1.05,1.15,1.25$, and 1.35 . These constants were used as the starting point for the sampling weights at the individual $\eta$ 's.

We next allowed the sampling weights to vary only for the particular cell where the beam was aimed:

$$
\chi^{2}=\sum_{j}\left[E_{0}-\left(\sum_{\text {cell } \neq \neq \text { beam }} E_{j}\right)-E_{\text {beameell }}\right]^{2} .
$$

$E_{j}$ is the energy in a cell for the $j$ th event and is given as above:

$$
E_{j}(\eta, \phi, \text { layer })=A(\text { detector }) * s(\eta, \text { layer }) * C(\eta, \phi, \text { layer }) * Q(\eta, \phi, \text { layer })
$$

Successive iterations were done, slowly improving the sampling weights at each $\eta$. The interplay between the three intercryostat region layers and between the various $\eta$ 's insured that the sampling weights converged to stable values very slowly. The convergence was considered complete when the sampling weights changed less than $1 \%$ from one iteration to the next. The final sampling weights for the three intercryostat detectors are listed in Table 5.5 . The first errors are statistical, the second errors are systematic and include the error in the MIP normalization constants and the error in the sampling weight determination.

The sampling weights are easily understood when considering the structure of the intercryostat region. First, where one or more of the detectors does not exist, the sampling weights of the remaining one(s) increase to pick up the extra energy. There is much more material in the $\eta$ region, $1.1<\eta<1.4$, due to the EC support ring and the curvature of the CC cryostat walls. As can be seen, the ICD sampling weights increase to pick up the extra energy lost in these support structures. These sampling weights for the Intercryostat Detector and the Massless Gaps were transferred over to $D \emptyset$ and used for $D \emptyset$ Run Ia (the first run of $D \emptyset, 05 / 92-05 / 93)$. 


\begin{tabular}{|c|c|c|c|}
\hline$\eta$ & CCMG & ICD & ECMG \\
\hline $0.70-0.80$ & $13.83 \pm 0.28 \pm 1.04$ & 0.000 & $10.50 \pm 0.22 \pm 0.79$ \\
\hline $0.80-0.90$ & $4.148 \pm 0.079 \pm 0.311$ & $14.60 \pm 0.28 \pm 1.10$ & $3.427 \pm 0.065 \pm 0.257$ \\
\hline $0.90-1.00$ & $6.548 \pm 0.119 \pm 0.491$ & $8.853 \pm 0.160 \pm 0.664$ & $3.306 \pm 0.060 \pm 0.248$ \\
\hline $1.00-1.10$ & $5.219 \pm 0.099 \pm 0.391$ & $24.82 \pm 0.47 \pm 1.86$ & $2.158 \pm 0.041 \pm 0.162$ \\
\hline $1.10-1.20$ & $3.546 \pm 0.068 \pm 0.266$ & $61.82 \pm 1.18 \pm 4.64$ & $4.109 \pm 0.078 \pm 0.308$ \\
\hline $1.20-1.30$ & 0.000 & $60.46 \pm 1.12 \pm 4.53$ & $1.756 \pm 0.032 \pm 0.132$ \\
\hline $1.30-1.40$ & 0.000 & $82.43 \pm 1.54 \pm 6.18$ & 0.000 \\
\hline
\end{tabular}

Table 5.5: The final sampling weights for the three intercryostat region detectors: the CC massless gap, the intercryostat detectors and the EC massless gaps.

\subsection{ICD Performance Results}

\subsubsection{Total Energy Determination}

Using the above sampling weights, we can see the improvements in detector response due to the intercryostat detector and the massless gaps. Figures 5.7 and 5.8 show the total calorimeter energy for $100 \mathrm{GeV} \pi$ 's, with and without the ICD and MG's. The response is shown for the central calorimeter at $\eta=0.45, \phi=31.5^{\circ}$, to provide a basis for comparison away from the intercryostat region; and for each of the seven intercryostat region $\eta$ 's. For the lower $\eta$ 's, the energy distribution without the intercryostat detectors is still Gaussian in shape, but the total energy is too low and the distribution is broader than the distribution obtained using information from the intercryostat detectors. If the ICR detectors were not present, the missing energy could be made up somewhat by optimizing the sampling weights of the calorimeter layers.

On the other hand, for the higher $\eta$ 's, there are two processes occurring. In the first, the particles are showering before the ICR and their energy is getting measured in the calorimeter, resulting in a small Gaussian peak at $100 \mathrm{GeV}$. For the other events, the particles do not shower until they reach the ICR and their energy is not sampled until they reach the end calorimeter, resulting in a long tail of particles with energy much less than the 
nominal $100 \mathrm{GeV}$. Without the ICR detectors, the overall energy measurement is severely degraded. The total calorimeter energy and the resolution $\left(\frac{\sigma}{E}\right)$ of the calorimeter are shown in Figure 5.9, with and without the ICR detectors.

\subsubsection{Linearity}

Using the above sampling weights, we can look at the linearity of response of the calorimeter as a function of energy. (The term "calorimeter" will now refer to all of $\mathrm{D} \emptyset$ calorimetry, including the central and end calorimeters, the massless gaps, and the intercryostat detector.) Figures $5.10,5.11,5.12,5.13$, and 5.14 show the reconstructed calorimeter energy as a function of the beam energy over the range from $10 \mathrm{GeV}$ to $100 \mathrm{GeV}$ at the indicated $\eta$ 's in the ICR. The straight line fit is indicated on the plots. Also shown is the fractional deviation from the fit. The error bars include the statistical error, the MIP normalization error and the error due to the optimization process. Figure 5.11 includes two different linearity scans at that $\eta$. The $\sim \pm 3 \%$ deviation indicates the accuracy of the linearity measurement. Overall, the calorimeter shows excellent linearity over the entire energy range. 

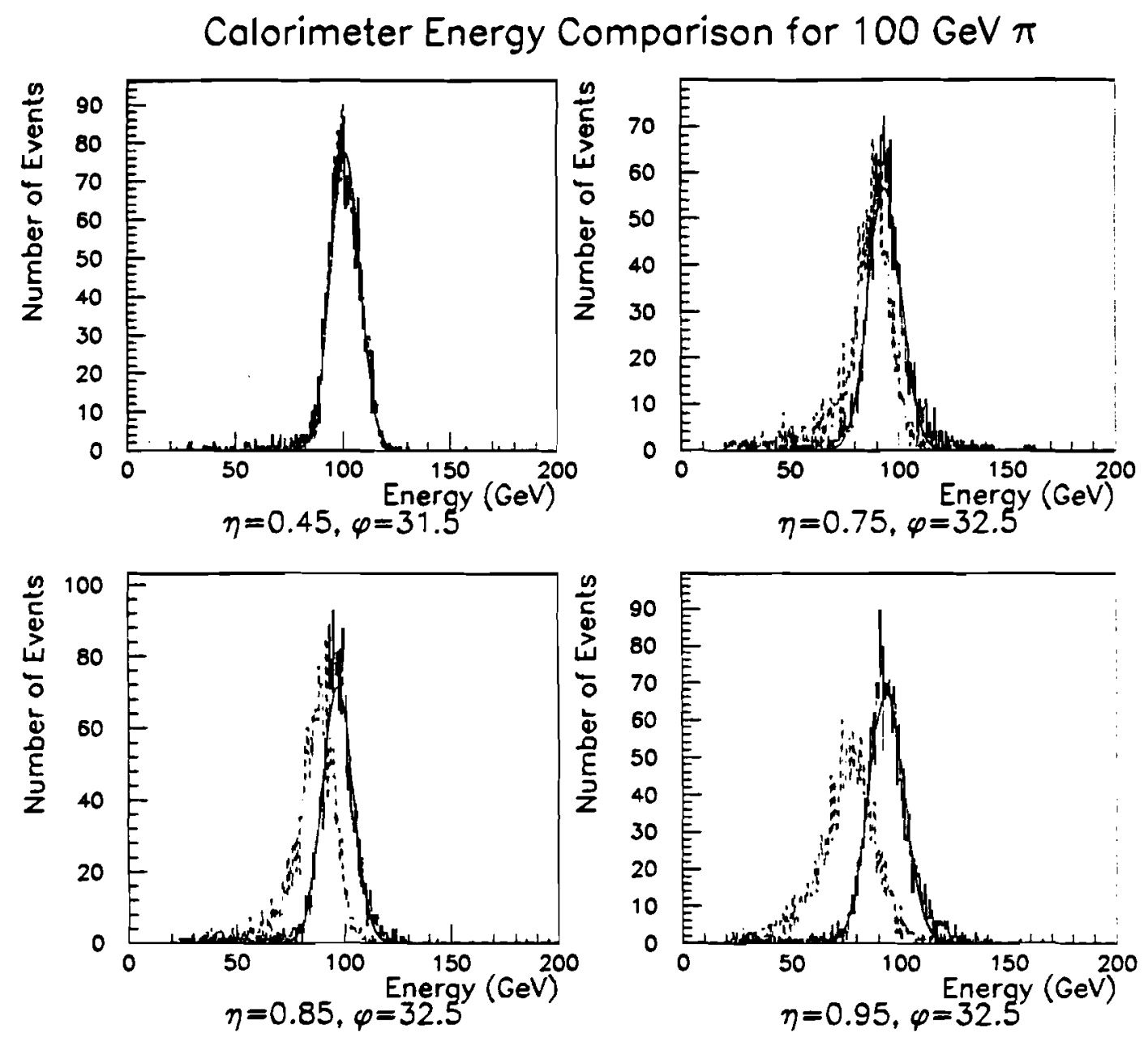

Figure 5.7: The total energy in the intercryostat region is shown, using the optimized sampling weights. The data is for $100 \mathrm{GeV}$ $\pi$ 's at the various ICR $\eta$ 's. For comparison, the calorimeter energy distribution with (solid line) and without the intercryostat region detectors (dashed line) is shown. A Gaussian fit to the total energy is also included. 
Calorimeter Energy Comparison for $100 \mathrm{GeV} \pi$
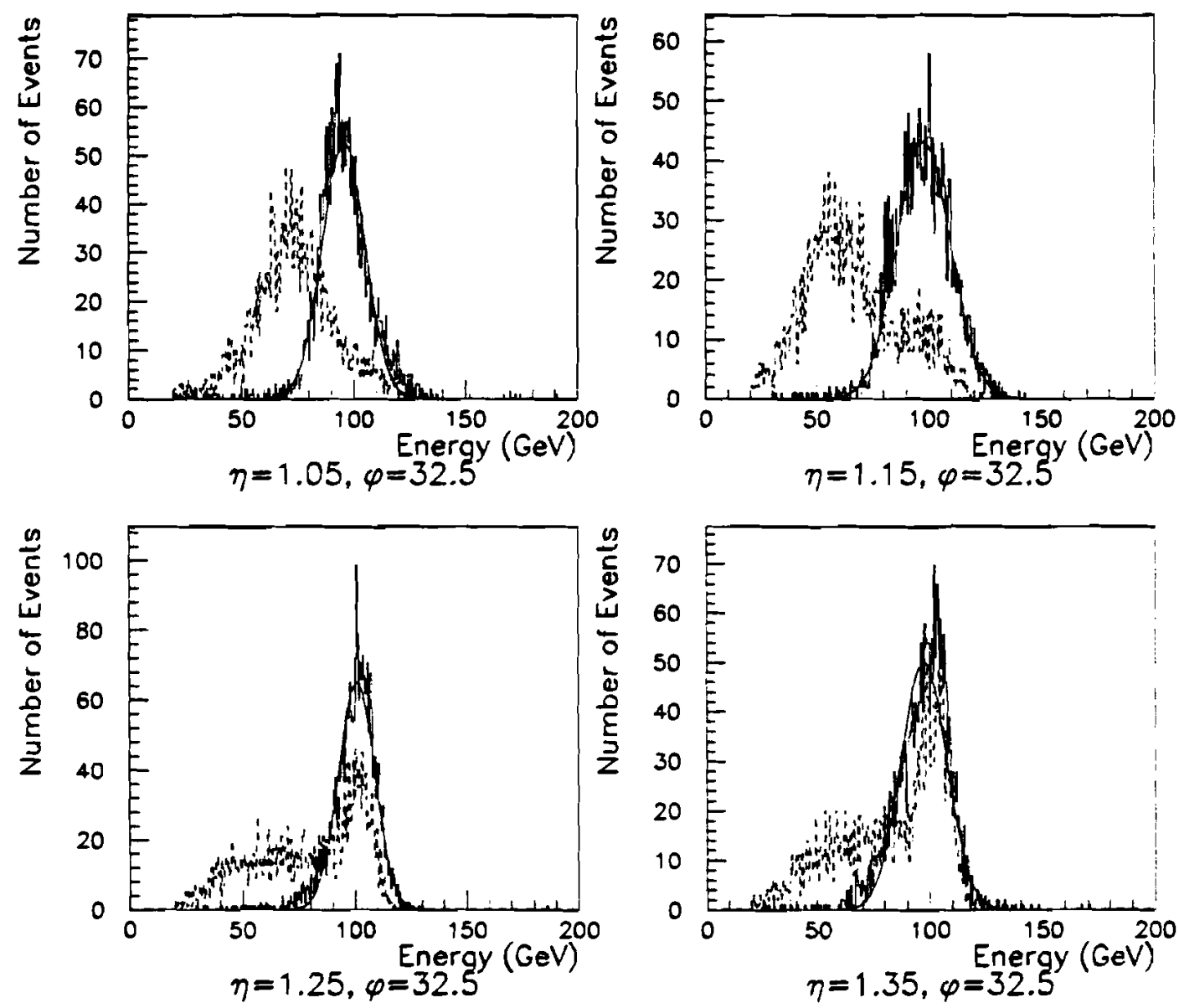

Figure 5.8: The total energy in the intercryostat region is shown, using the optimized sampling weights. The data is for $100 \mathrm{GeV}$ $\pi$ 's at the various ICR $\eta$ 's. For comparison, the calorimeter energy distribution with (solid line) and without the intercryostat region detectors (dashed line) is shown. A Gaussian fit to the total energy is also included. 

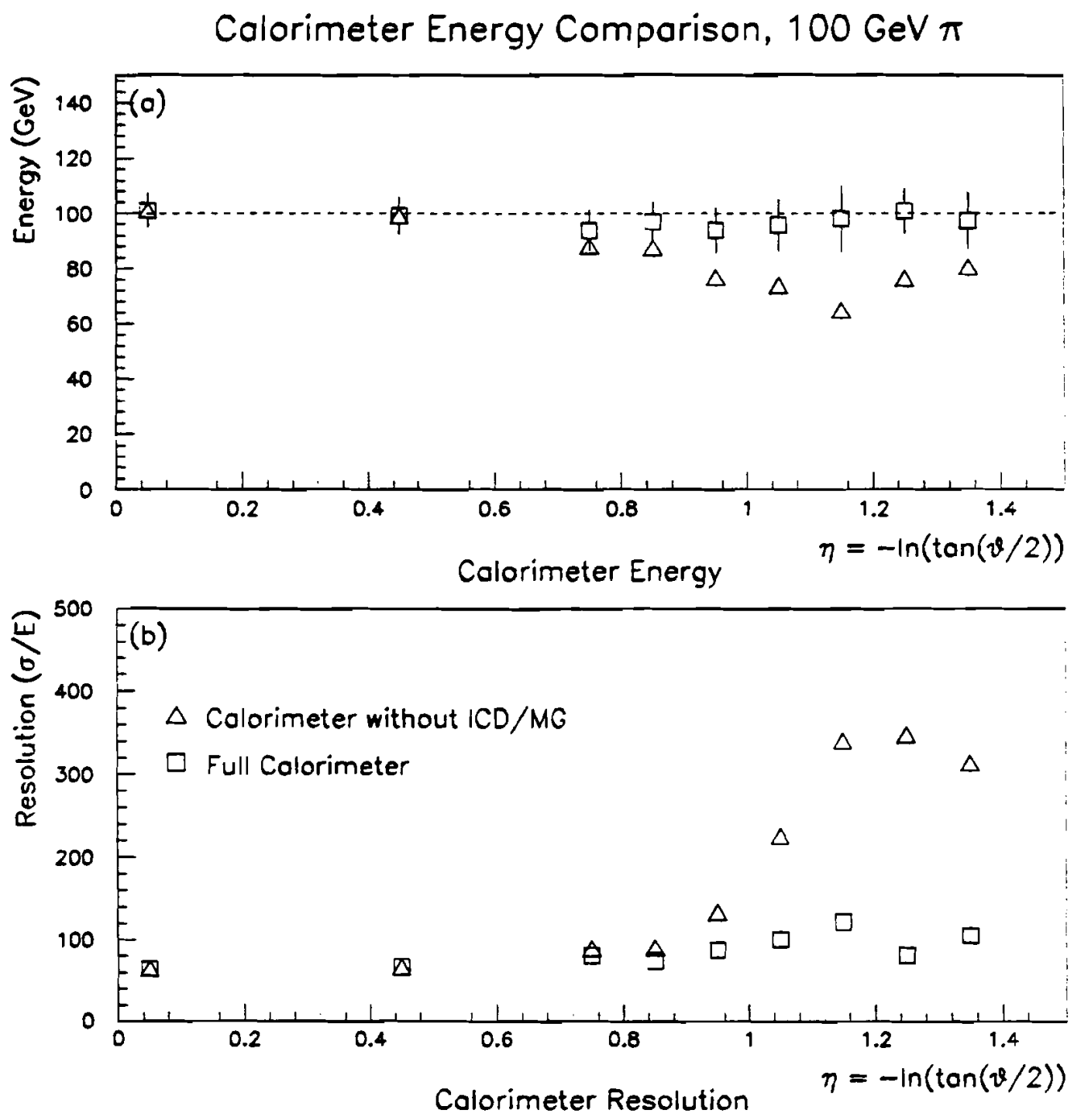

Figure 5.9: The (a) calorimeter energy and (b) resolution in the intercryostat region is shown as a function of $\eta$. The data is for $100 \mathrm{GeV} \pi$ 's. The result with and without the intercryostat region detectors is indicated. 


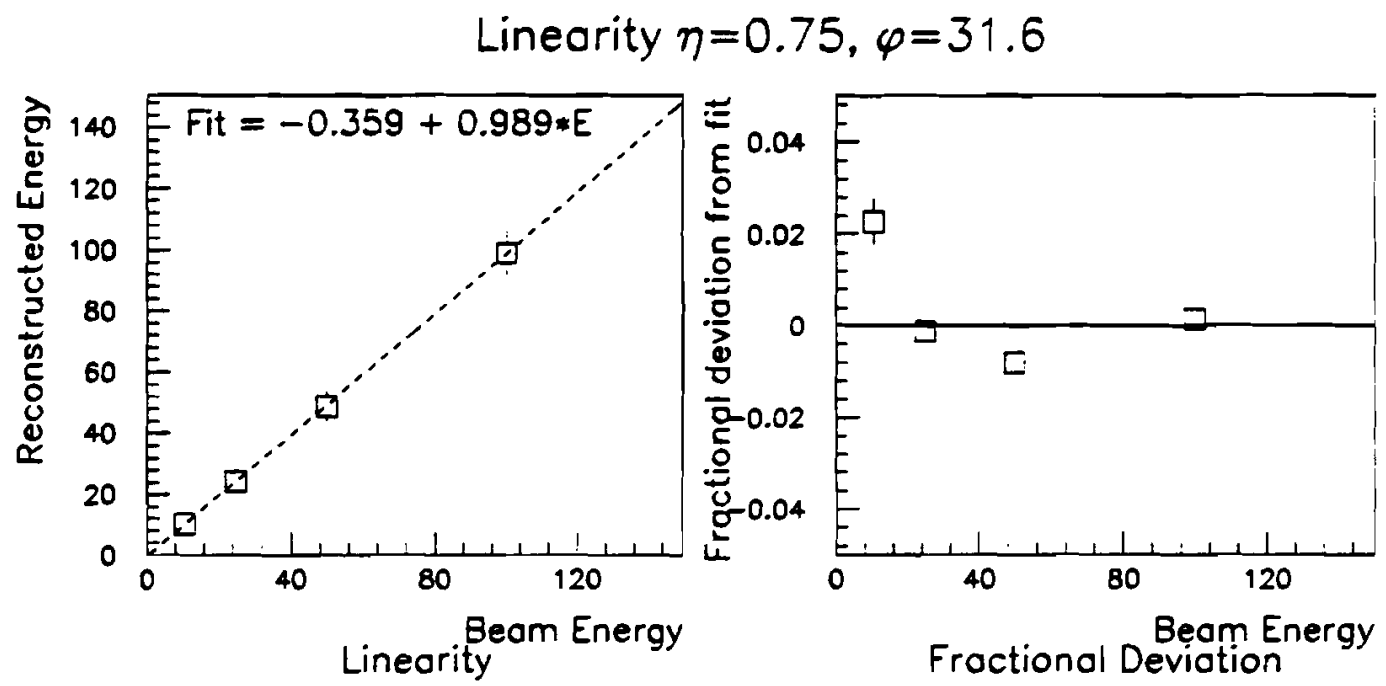

Figure 5.10: The reconstructed calorimeter energy is plotted as a function of beam momentum for $\eta=0.75, \phi=31.6$. The best fit is noted on the plot. The fractional deviation from the straight line fit is also shown.

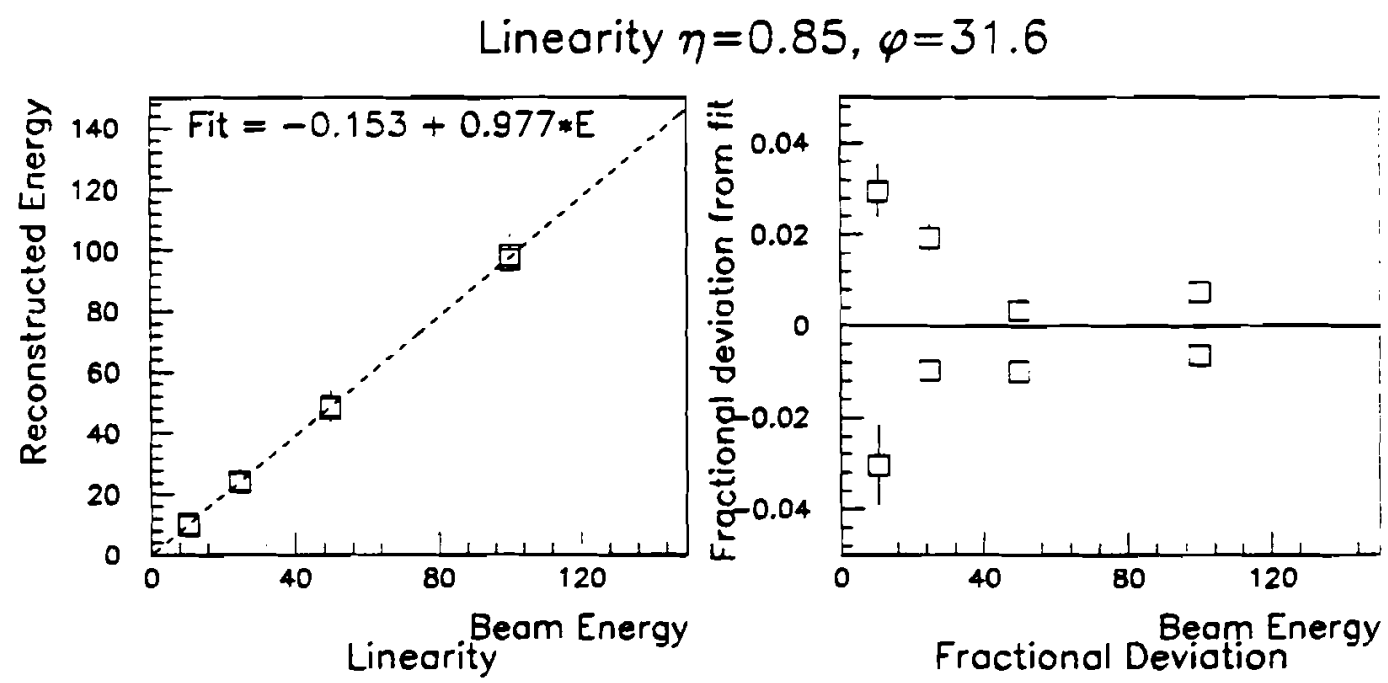

Figure 5.11: The reconstructed calorimeter energy is plotted as a function of beam momentum for $\eta=0.85, \phi=31.6$. The best fit is noted on the plot. The fractional deviation from the straight line fit is also shown. 
Linearity $\eta=0.95, \varphi=31.6$
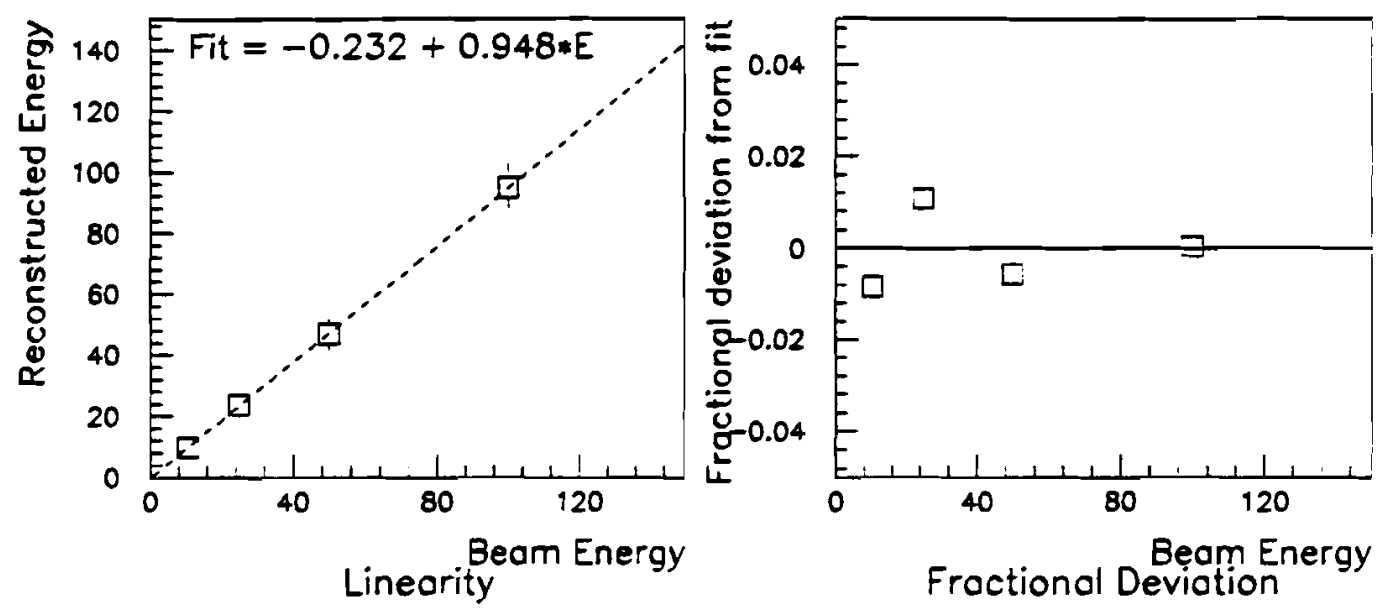

Figure 5.12: The reconstructed calorimeter energy is plotted as a function of beam momentum for $\eta=0.95, \phi=31.6$. The best fit is noted on the plot. The fractional deviation from the straight line fit is also shown.

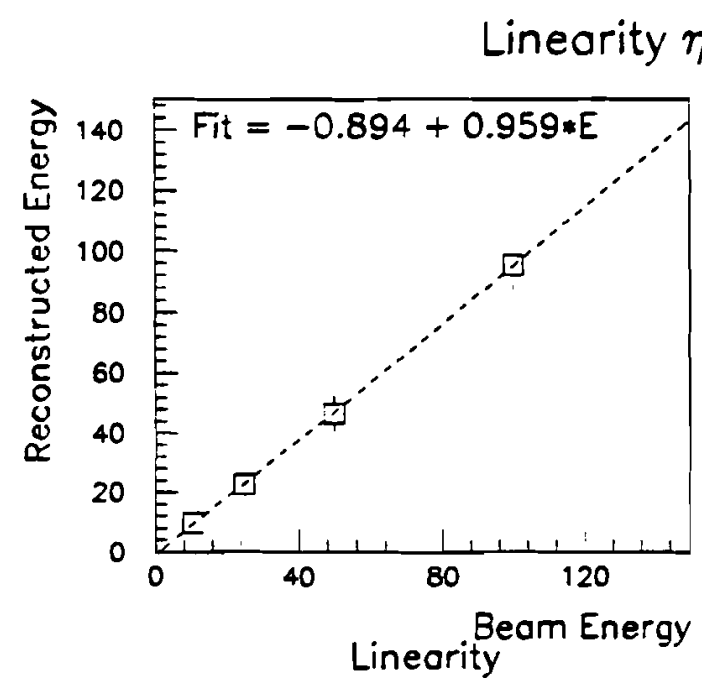

$\eta=1.05, \varphi=31.6$

Figure 5.13: The reconstructed calorimeter energy is plotted as a function of beam momentum for $\eta=1.05, \phi=31.6$. The best fit is noted on the plot. The fractional deviation from the straight line fit is also shown. 


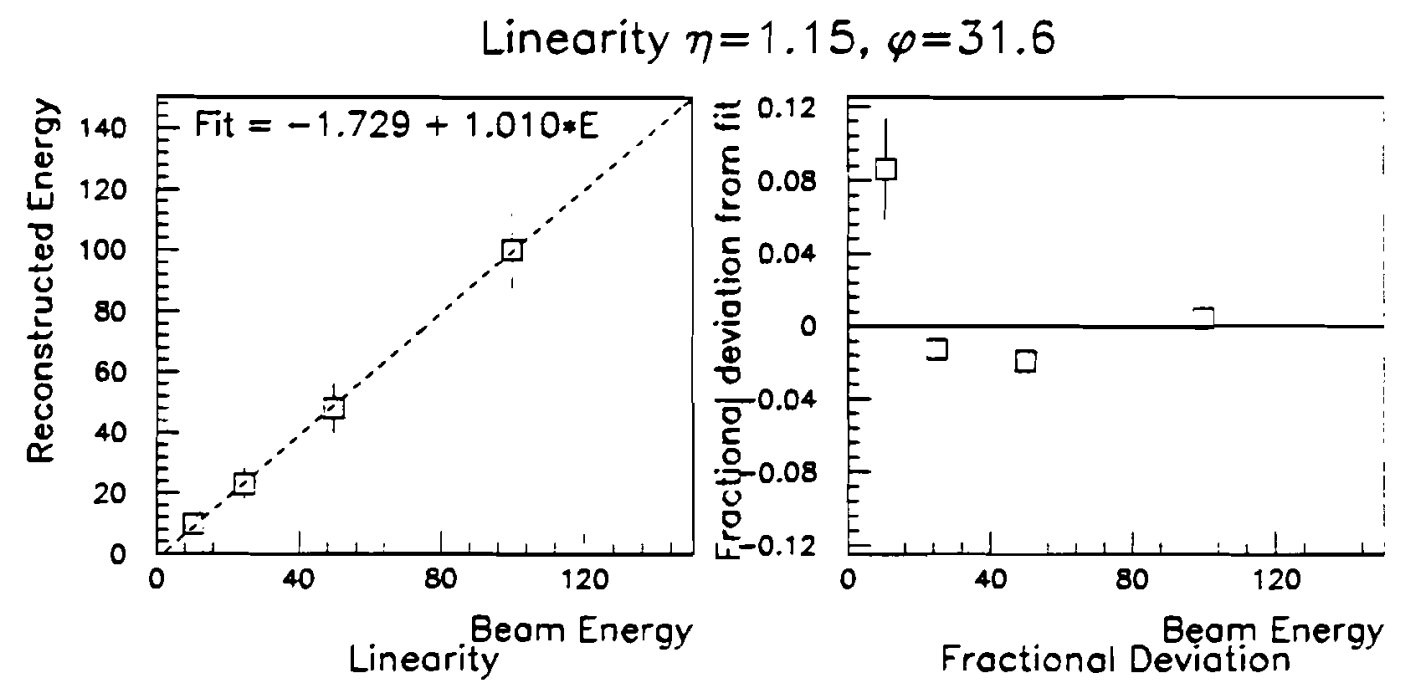

Figure 5.14: The reconstructed calorimeter energy is plotted as a function of beam momentum for $\eta=1.15, \phi=31.6$. The best fit is noted on the plot. The fractional deviation from the straight line fit is also shown. 


\section{CHAPTER 6}

\section{THE TEST BEAM MONTE CARLO SIMULATION}

\subsection{Overview}

The 1991 Load II test beam was simulated using the standard GEANT detector simulation package [62], originally developed at CERN. Monte Carlo simulations are often used to aid in designing a detector or to gain better understanding of an existing detector. In the case of the test beam detector, we can hope to use a Monte Carlo simulation to verify the calibration from the test beam detector and to give us confidence in the sampling weights determined from the test beam data.

\subsection{Description of General Simulation}

GEANT is a detector and physics simulation package. Using GEANT, one can describe the detector geometry in a very detailed manner. The geometry simulation is based on defining the individual volumes which make up a detector. Simulated particles then pass through each of these volumes, depositing energy according to simulated physics processes, such as Bremsstrahlung, Coulomb scattering, $\delta$-ray production, particle decays and showering of electromagnetic or hadronic particles.

The test beam load was very carefully reproduced in the Monte Carlo simulation. For the calorimeter modules, each individual argon gap, absorber plate, signal board, and readout board have been accurately simulated. Dead material, such as support structures, was also included. The test beam cryostat was modelled, as were the beamline materials, such as the PWC's, scintillator paddles and Cerenkov counters. 
The test beam simulation is a plate-level simulation, i.e., each actual volume or plate is modelled, down to the thinnest layer of copper. Conversely, the full DØ GEANT is a mixture-level simulation. In the mixture simulation, a new material is defined to be the weighted average of all the constituent materials in the calorimeter. This material is then used to fill the appropriate volume. The disadvantage of the plate level simulation is that each event takes much more computer time to generate than the mixture level. However, the shower shapes are more accurate using the plate level geometry. The geometry of the test beam simulation can be seen in Figure 6.1.

\subsubsection{Description of Calorimeter Simulation}

The basic module volume of the central calorimeter modules is a polycone which holds all the active and inactive support structures [63]. Inside the polycone is a polygon containing all the active media. The polygon is filled with liquid argon. A single basic cell is used for the entire CC. The cell consists of the absorber, the first argon gap, the signal board (including the copper pads), the second argon gap, and the readout board. Each of these volumes is a plate of the appropriate thickness. Each of the absorber plates, argon gaps, signal boards, and readout boards was modelled as an individual plate.

In order to compare test beam data with the test beam Monte Carlo simulation, it was necessary to first calculate the appropriate sampling fractions for the various modules in the Monte Carlo simulation. These were true sampling fractions (not sampling weights), calculated by using the standard $d E / d x$ energy lost by a minimum ionizing particle going through each of the detector elements. The sampling fraction is then given by:

$$
s f=\frac{E_{\text {live }}}{E_{\text {dead }}+E_{\text {live }}} .
$$

The sampling fractions for each of the modules in the test beam Monte Carlo simulation are listed in Table 6.1, along with the $d E / d x$ sampling fractions for the real $\mathrm{D} \emptyset$ modules for comparison.

\subsubsection{Description of Intercryostat Region Simulation}

The simulation of the intercryostat region was also done as accurately as possible. The dummy steel walls were simulated by breaking them into separate pieces which could be 


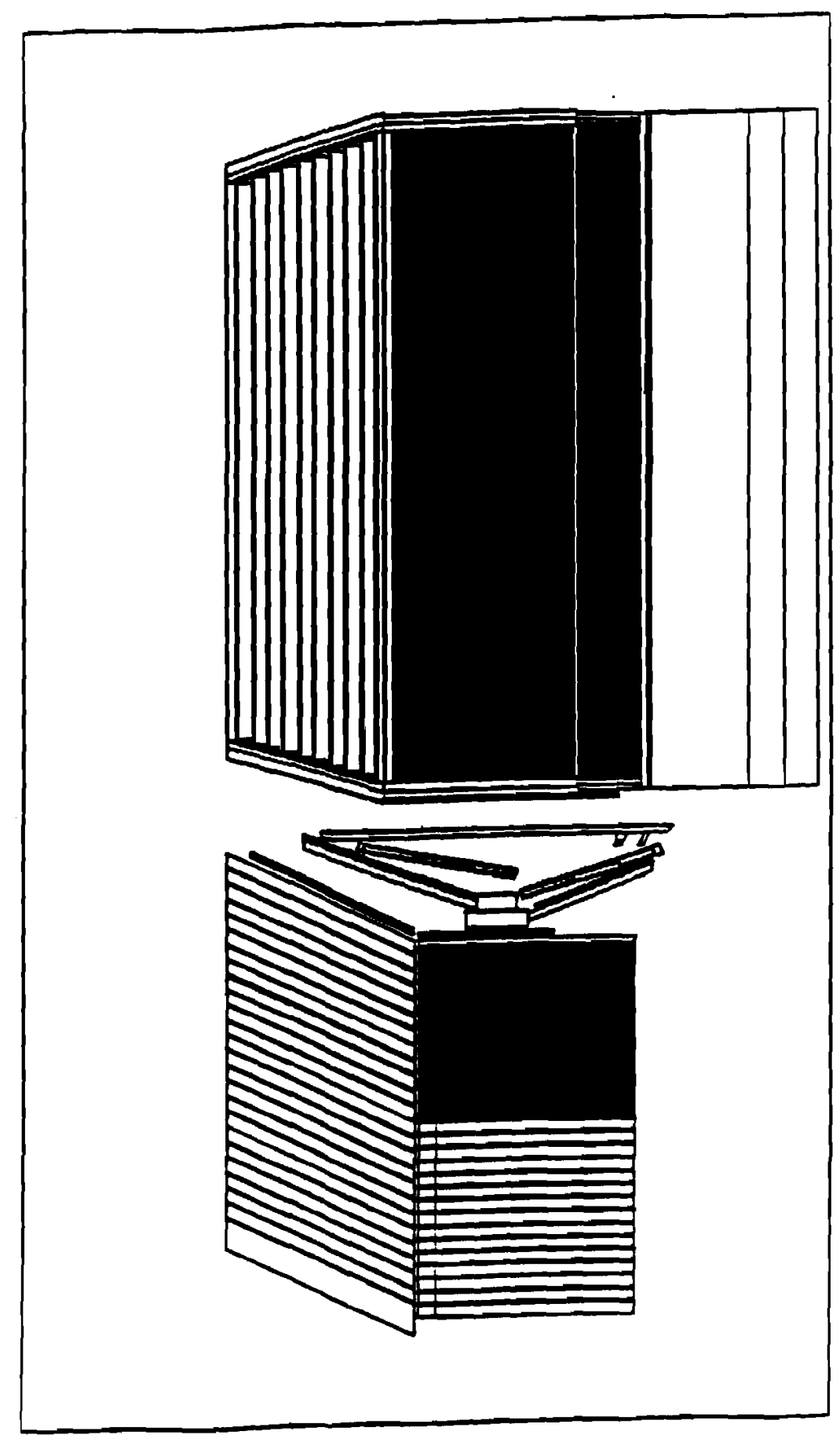

Figure 6.1: A plan view of the test beam Monte Carlo simulation geometry. The central calorimeter electromagnetic and fine hadronic modules and the massless gaps appear as completely shaded in because there are many different layers, such as argon gaps and uranium plates, packed into a very small space. 


\begin{tabular}{|l|r|r|}
\hline Detector & D0 samp. frac. & MC samp. frac. \\
\hline CCEM1 & $10.94 \%$ & $9.14 \%$ \\
\hline CCEM2 & $11.97 \%$ & $12.00 \%$ \\
\hline CCEM3 & $11.90 \%$ & $12.00 \%$ \\
\hline CCEM4 & $12.35 \%$ & $12.20 \%$ \\
\hline CCFH1 & $6.59 \%$ & $6.42 \%$ \\
\hline CCFH2 & $6.90 \%$ & $6.71 \%$ \\
\hline CCFH3 & $6.89 \%$ & $6.70 \%$ \\
\hline CCCH & $1.45 \%$ & $1.46 \%$ \\
\hline ECMFH1 & $6.33 \%$ & $6.27 \%$ \\
\hline ECMFH2 & $6.92 \%$ & $6.79 \%$ \\
\hline ECMFH3 & $6.90 \%$ & $6.79 \%$ \\
\hline ECMFH4 & $6.90 \%$ & $6.79 \%$ \\
\hline ECMCH & $1.64 \%$ & $1.57 \%$ \\
\hline ECOCH1 & $1.64 \%$ & $1.74 \%$ \\
\hline ECOCH2 & $1.64 \%$ & $1.74 \%$ \\
\hline ECOCH3 & $1.64 \%$ & $1.35 \%$ \\
\hline
\end{tabular}

Table 6.1: The $d E / d x$ sampling fractions for the modules in the test beam Monte Carlo simulation. The sampling fractions for the real modules are listed for comparison. The laver column refers to the detector software label and will be discussed later. 
described by existing GEANT volumes. Their material was iron. The walls were positioned by using the test beam survey information. Survey marks on the walls were related to the positions of the calorimeter modules.

Two aluminum boxes, high eta and low eta, were created using GEANT trapezoidal volumes and filled with liquid argon. Five copies were made of each of these boxes and the boxes were positioned with respect to the walls using information from our own survey of the boxes during installation. Similarly, each of the six different sizes of tiles was constructed using a scintillator-filled trapezoid. These were then copied five times and placed in each of the five boxes. The test beam cryostat volume was filled with excluder in the Monte Carlo simulation. Thus it was not necessary to create the individual excluder volumes. A close-up view of the intercryostat region is shown in Figure 6.2. The ICD tiles can be seen, nestled inside their box volumes.

\subsection{Determination of Calorimeter Normalization Constants}

The response of a calorimeter to an incident particle is a very complicated process [64]. It depends on the type of particle, the material and thickness of the absorber, the material and thickness of the active medium, the amount of energy deposited in the active medium, and the ionization density of the active medium. The calorimeter response to a minimum ionizing particle will be much different than the response to an electron or non-minimum ionizing proton depositing the same amount of energy. In the calculation of energy for the real detector, these differences are buried in the sampling weights and the ADC-to-GeV constants. However, for the Monte Carlo data, these differences must be explicitly taken into account.

The total energy seen in the Monte Carlo simulation detector will be:

$$
\mathrm{E}=\sum_{j} E_{j}(\eta, \phi, \text { layer }) * s f(\text { layer }) / \frac{(e \text { or } \pi)}{\operatorname{mip}}
$$

where $E_{j}$ is the energy deposited in a cell, the sampling fractions are given above and the $\frac{e}{m i p}$ and $\frac{\pi}{m i p}$ are constants which take into account the difference in calorimeter response to an electron or pion versus a minimum ionizing particle. As we are looking at hadrons in the real data, $\frac{\pi}{\operatorname{mip}}$ is the important constant.

The determination of $\frac{\pi}{m i p}$ is based on certain assumptions. These constants are most strongly dependent on the absorber material ( $Z$ of the absorber) and are only weakly de- 

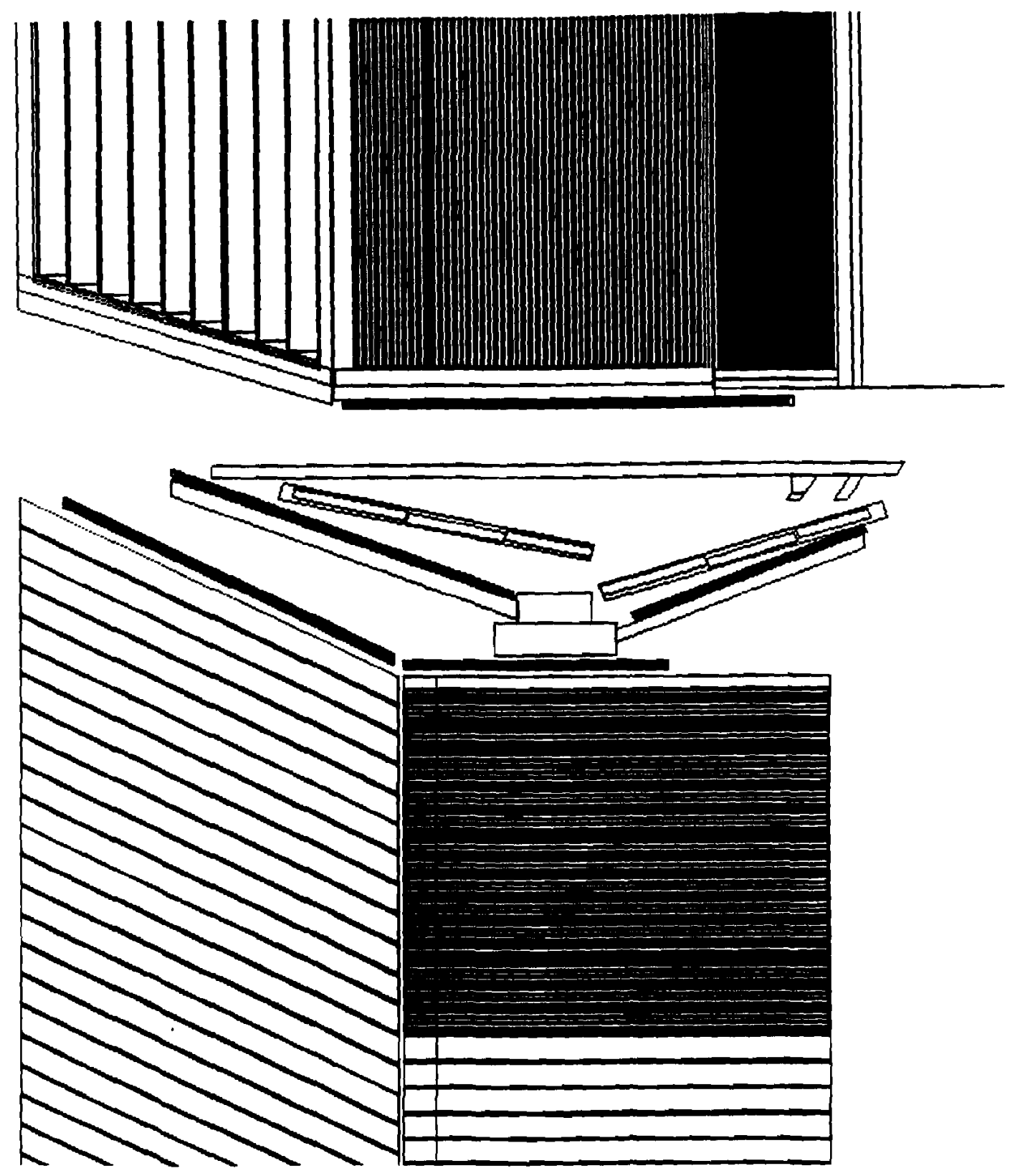

Figure 6.2: A plan view of the intercryostat region in the test beam Monte Carlo simulation geometry. The massless gaps appear as completely shaded in because there are many different layers packed into a very small space. However, the differentiation between the layers can now be seen for the electromagnetic and fine hadronic modules. 
pendent on the thickness of the absorber (and only for very thin, $\leq 3 \mathrm{~mm}$, plates). The CCEM, CCFH and ECMFH use uranium as the absorber $(Z=92)$; the FH uranium plates are $6 \mathrm{~mm}$ thick, the EM plates are $3 \mathrm{~mm}$ thick. The $\mathrm{CCCH}$ uses $46.5 \mathrm{~mm}$ copper absorber plates $(Z=29)$; the ECMCH and ECOCH use $46.5 \mathrm{~mm}$ steel absorber plates $(Z=26)$. The most obvious choice is one $\frac{\pi}{m i p}$ constant for all uranium calorimetry (CCEM, CCFH and ECMFH), one constant for the copper ( $\mathrm{CCCH}$ ) and one for the steel calorimetry (ECMCH and $\mathrm{ECOCH})$.

The $\frac{\pi}{m i p}$ constants for the uranium and copper calorimetry were determined by optimizing the calorimeter response to $100 \mathrm{Gev} \pi$ 's at $\eta=0.45, \phi=31.5^{\circ}$. The constant for the steel calorimetry was found by optimizing the calorimeter response at $\eta=1.05, \phi=32.5^{\circ}$, as will be noted below.

\subsection{Comparison of Monte Carlo Data with Test Beam Data}

The Monte Carlo simulation data was generated with very low cutoffs, $10 \mathrm{keV}$ for electrons and photons; $100 \mathrm{keV}$ for hadrons. Approximately 500 events were generated for each point. The simulation data was subjected to the same cuts as the real data, where relevant. In particular, a $17 \times 17(\Delta \eta \times \Delta \phi)$ road around the beam was used in the analysis; $100 \mathrm{GeV}$ $\pi$ data was used and leakage events were discarded. The sampling fractions used were the ones calculated for the simulation modules, as mentioned above. The simulation particles, being a monoenergetic beam travelling down a perfect beamline, did not suffer from such effects as mistuned beams or poorly reconstructed tracks.

Sampling fractions were determined for the intercryostat region detectors in an analogous fashion to the determination of the sampling weights for the real detectors. A $\chi^{2}$ optimization technique was used to optimize the total energy. A slight modification was introduced due to the $\frac{\pi}{m i p}$ constant for the EC coarse hadronic (steel absorber) modules. That constant was first set equal to the nominal value for the copper absorber and a set of sampling fractions determined for the ICR. The steel $\frac{\pi}{m i p}$ constant was then determined at $\eta=1.05, \phi=32.5^{\circ}$, the $\eta$ with the maximum energy deposition in that layer. The optimization was then redone to obtain the final set of ICR sampling fractions.

The most straight-forward method for comparison of Monte Carlo simulation data and test beam data is to look at the fractional energy in each layer of the detector. The detector readout layers are labelled from 1 to 17 , as one moves radially outward from the interaction 


\begin{tabular}{|c|c|}
\hline Layer & Detectors \\
\hline 1 & CCEM1 \\
\hline 2 & CCEM2 \\
\hline $3-6$ & CCEM3 \\
\hline 7 & CCEM4 \\
\hline 8 & CCMG \\
\hline 9 & ICD \\
\hline 10 & ECMG \\
\hline 11 & CCFH1, ECMFH1 \\
\hline 12 & CCFH2, ECMFH2 \\
\hline 13 & CCFH3, ECMFH3 \\
\hline 14 & ECMFH4 \\
\hline 15 & CCCH, ECMCH, ECOCH \\
\hline 16 & ECOCH2 \\
\hline 17 & ECOCH3 \\
\hline
\end{tabular}

Table 6.2: The readout layers and corresponding detector layers are listed. 
point. The layers and their corresponding detectors are listed in Table 6.2. The third CCEM layer actually consists of four separate readouts because of the finer $\Delta \eta \times \Delta \phi$ segmentation $(0.5 \times 0.5)$ and is labelled as layers 3-6. For comparison purposes, all the energy in the CCEM3 layer has been added together into layer 3. The first figure, Figure 6.3(a), plots the fractional energy of each layer $\left(E_{\text {layer }} / E_{\text {total }}\right.$ ) as a function of layer number for $100 \mathrm{GeV} \pi$ 's at $\eta=0.45, \phi=31.5^{\circ}$, where the central calorimeter $\frac{\pi}{m i p}$ constants were determined. The errors on the data are determined by the errors on the sampling weights (statistical and systematic). The errors on the Monte Carlo data are purely statistical. Also shown in plot (b) of that figure is the difference between the layer energy in data and the layer energy in the Monte Cario simulation. Figures 6.4, 6.5, 6.6, 6.7, 6.8, 6.9, 6.10, and 6.11 are the same comparison plots for $\eta=0.05$ and $\eta=0.75-1.35$.

The layer energies predicted by the Monte Carlo simulation generally follow the trend of the layer energies from the data, shown in the (a) plots of the comparison figures. From the (b) plots, we see that there are, however, basic differences at the few $\mathrm{GeV}$ (out of $100 \mathrm{GeV}$ ) level. These differences exist even at the calorimeter only $\eta$ 's of 0.45 (where the $\frac{\pi}{m i p}$ optimization was done) and 0.05 , where the ICD and MG's do not exist. The calorimeter energy differences make optimization of the intercryostat region layers difficult and generally, as shown in Figure 6.12, the ICR layers do not make up the full $100 \mathrm{GeV}$ input energy. Figure 6.12(a) compares the total energy in data and simulation and (b) detector resolution, as a function of $\eta$. The error bars in (a) are the widths from the Gaussian fit to the total energy. It is obvious that further improvements to the calorimeter and intercryostat region of the Monte Carlo simulation are necessary to obtain agreement at the few percent level, before we can directly use the sampling fractions predicted by the Monte Carlo simulation for $D \emptyset$. Nonetheless, the current Monte Carlo simulation has allowed us to gain general confidence in the test beam results.

Before we proposed to implement the ICD in the test beam run, there were many discussions about how to calibrate the detector response. Monte Carlo simulation studies were one such method discussed and there were several attempts at using the full D $\emptyset$ GEANT simulation to determine optimum sampling weights (see for example [65]). In retrospect, those methods incorrectly predicted less energy in the ICR detectors than determined via the test beam run. The inclusion of the ICD in the test beam run was therefore vitally necessary in order to calibrate the detector before the start of the full $\mathrm{D} \emptyset$ collider run. 


\section{Fractional Energy Comparison, $100 \mathrm{GeV} \pi, \eta=0.45$}
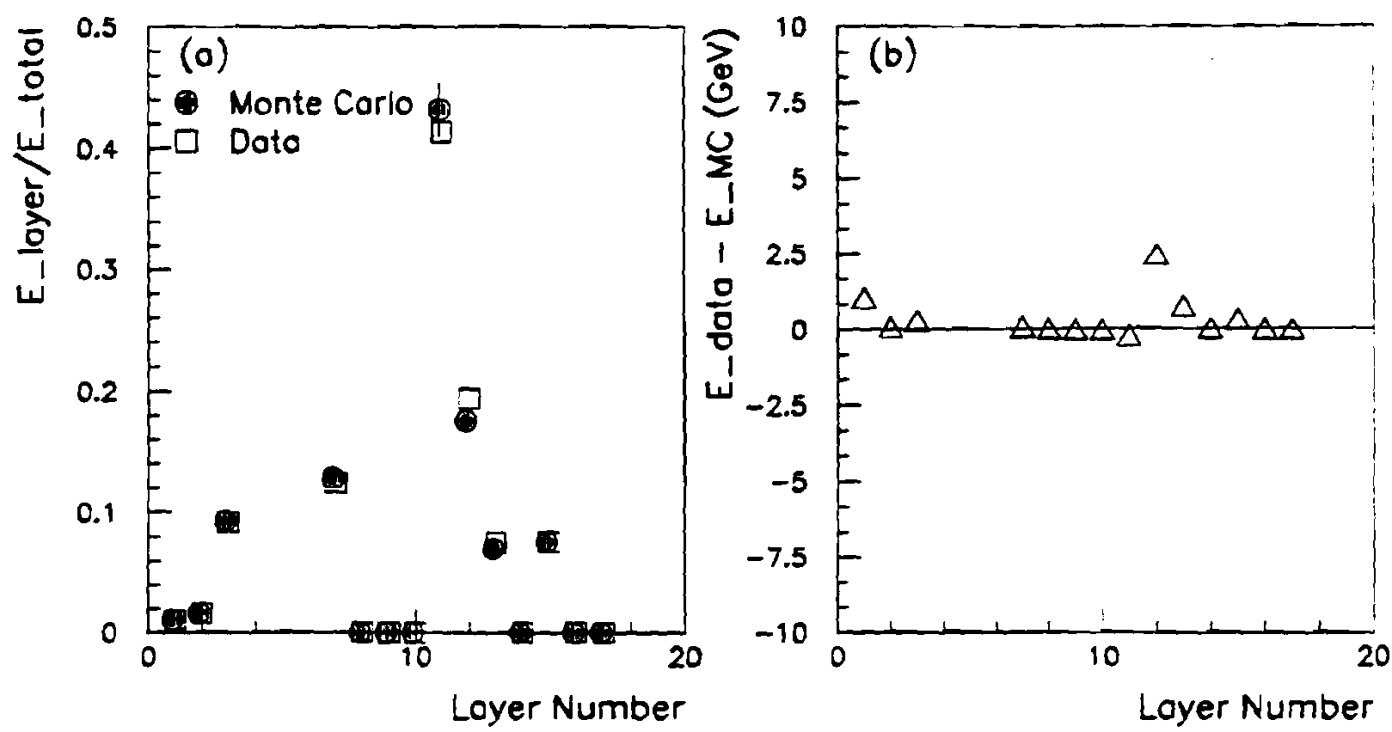

Figure 6.3: A comparison between test beam data with Monte Carlo simulation data at $\eta=0.45$. (a) shows the fractional energy in each readout layer for data and simulation. (b) shows the difference between the energy in each layer for the Monte Carlo simulation data and the real data.

Froctional Energy Comparison, $100 \mathrm{GeV} \pi, \eta=0.05$
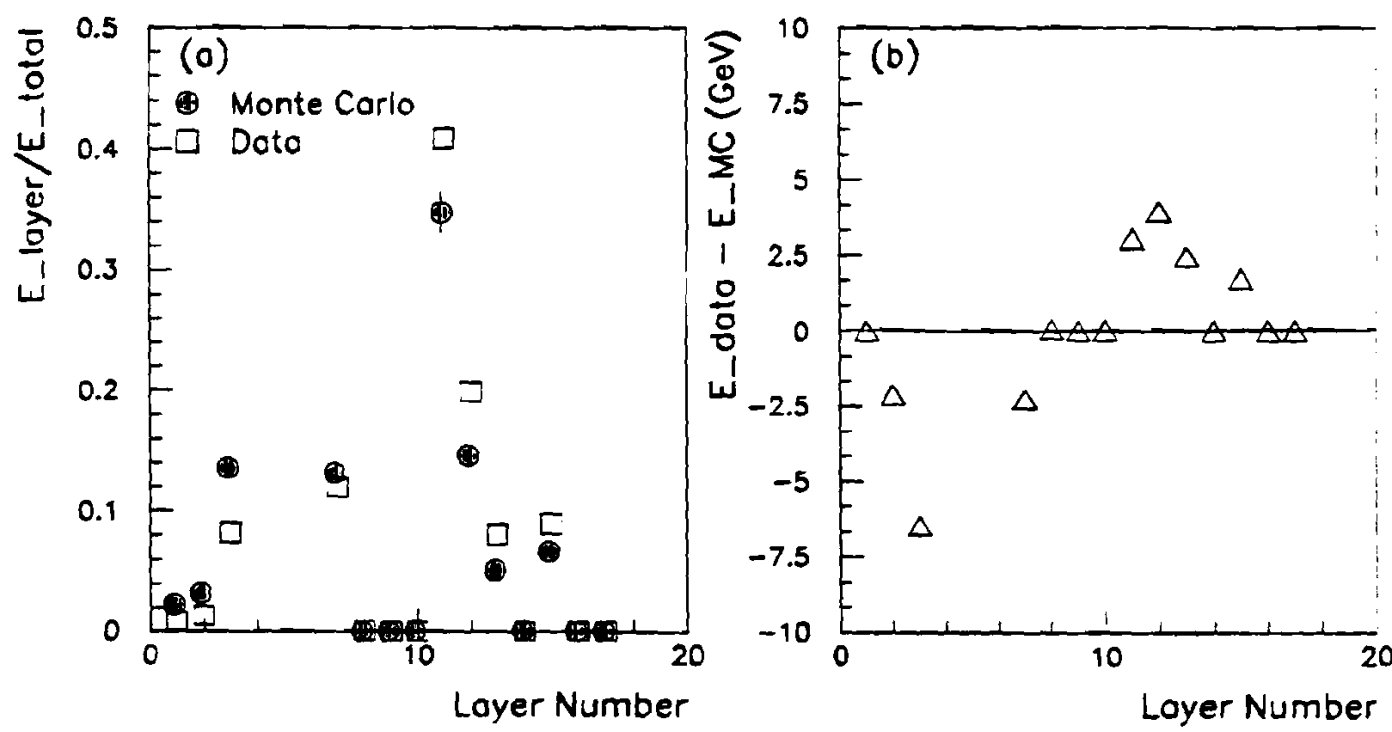

Figure 6.4: A comparison between test beam data with Monte Carlo simulation data at $\eta=0.05$. (a) shows the factional energy in each readout layer for data and simulation. (b) shows the difference between the energy in each layer for the Monte Carlo simulation data and the real data. 
Fractional Energy Comparison, $100 \mathrm{GeV} \pi, \eta=0.75$
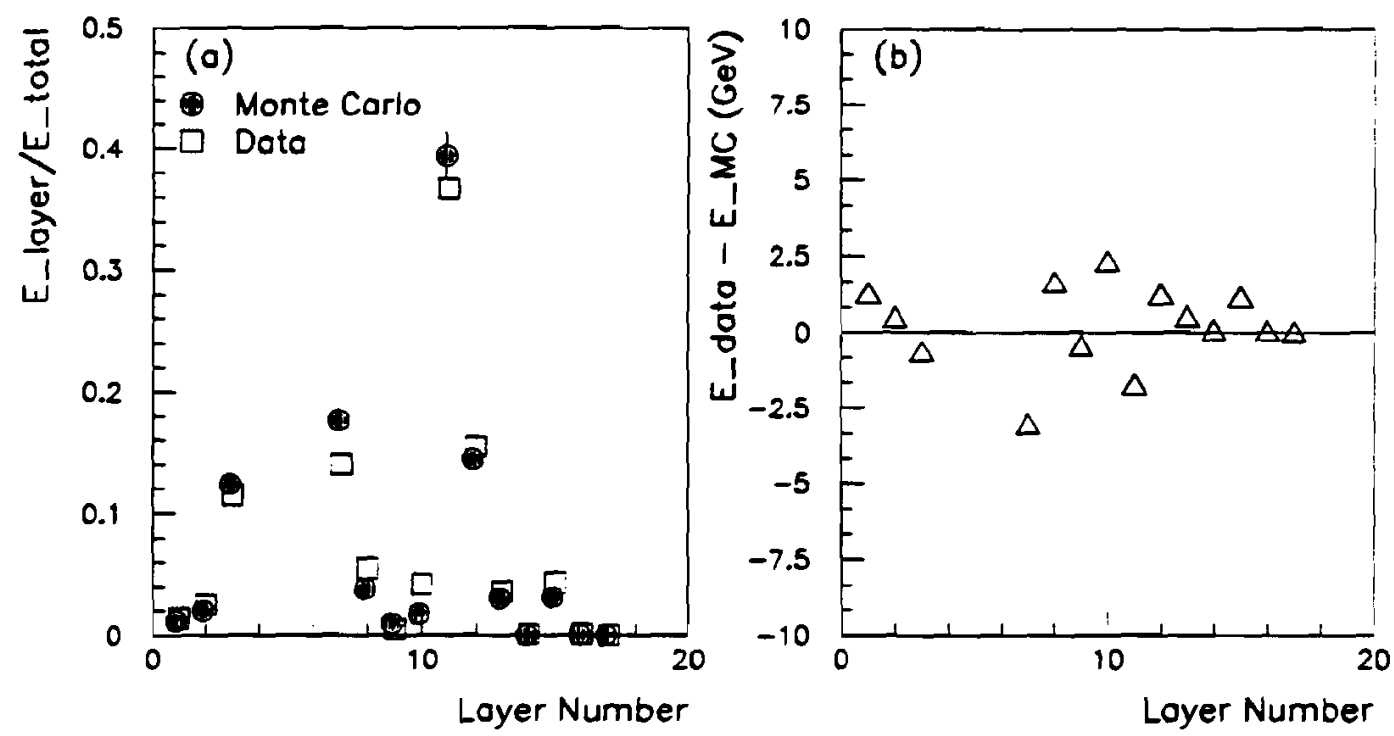

Figure 6.5: A comparison between test beam data with Monte Carlo simulation data at $\eta=0.75$. (a) shows the fractional energy in each readout layer for data and simulation. (b) shows the difference between the energy in each layer for the Monte Carlo simulation data and the real data.

Fractional Energy Comparison, $100 \mathrm{GeV} \pi, \eta=0.85$
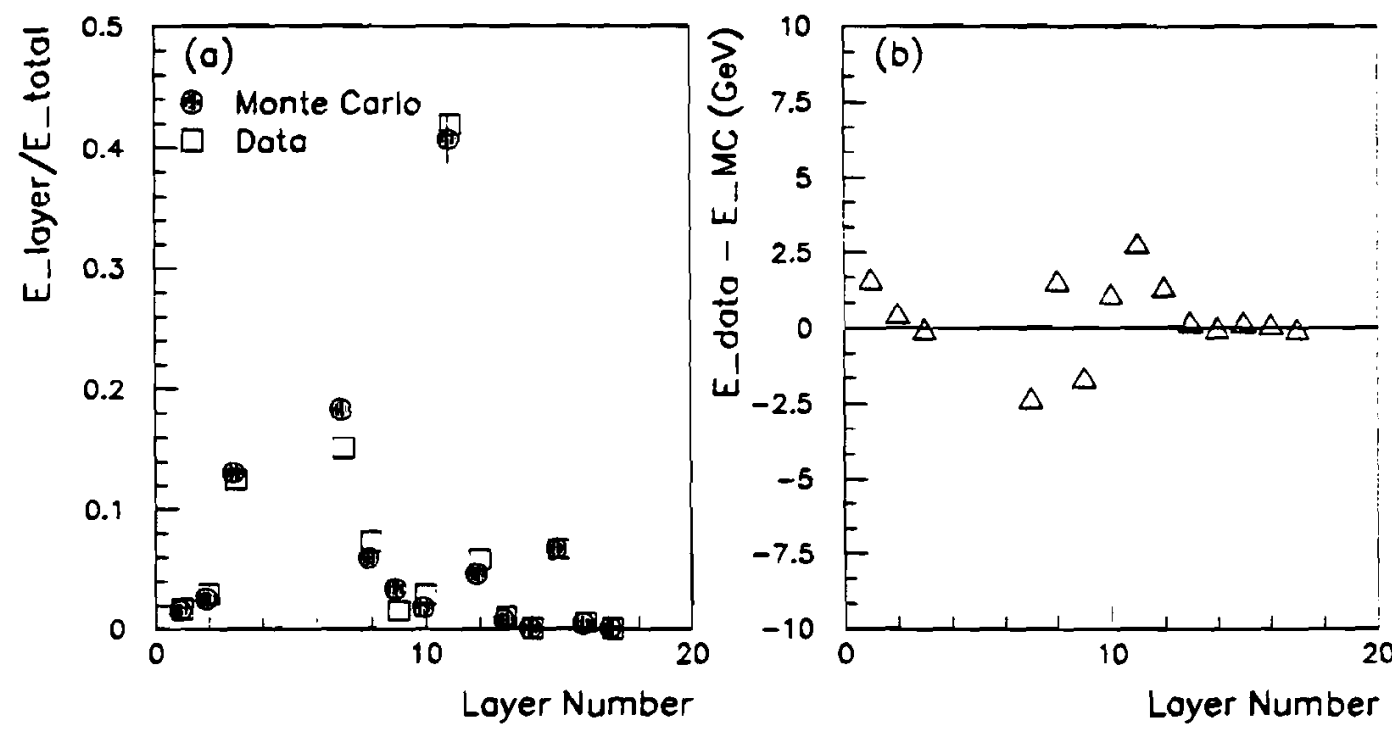

Figure 6.6: A comparison between test beam data with Monte Carlo simulation data at $\eta=0.85$. (a) shows the fractional energy in each readout layer for data and simulation. (b) shows the difference between the energy in each layer for the Monte Carlo simulation data and the real data. 
Fractional Energy Comparison, $100 \mathrm{GeV} \pi, \eta=0.95$
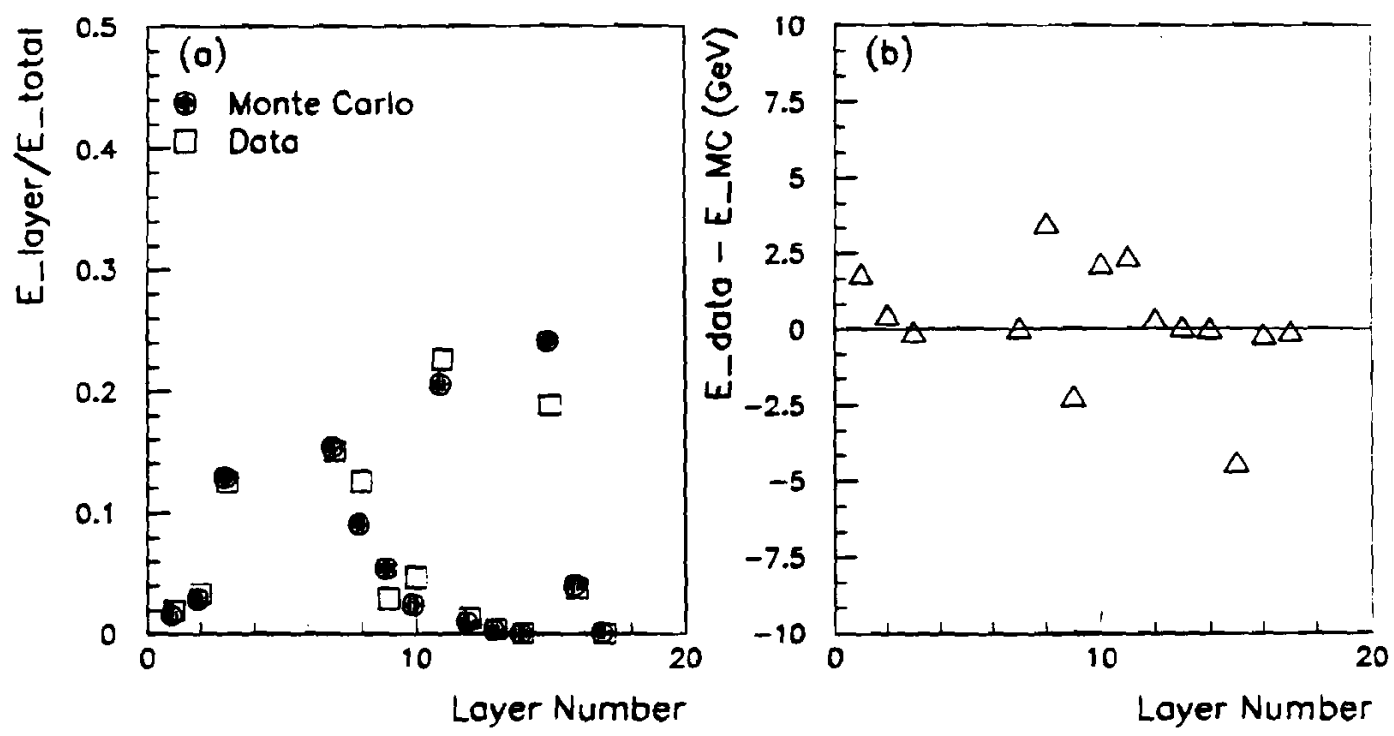

Figure 6.7: A comparison between test beam data with Monte Carlo simulation data at $\eta=0.95$. (a) shows the fractional energy in each readout layer for data and simulation. (b) shows the difference between the energy in each layer for the Monte Carlo simulation data and the real data.

Fractional Energy Comparison, $100 \mathrm{GeV} \pi, \eta=1.05$
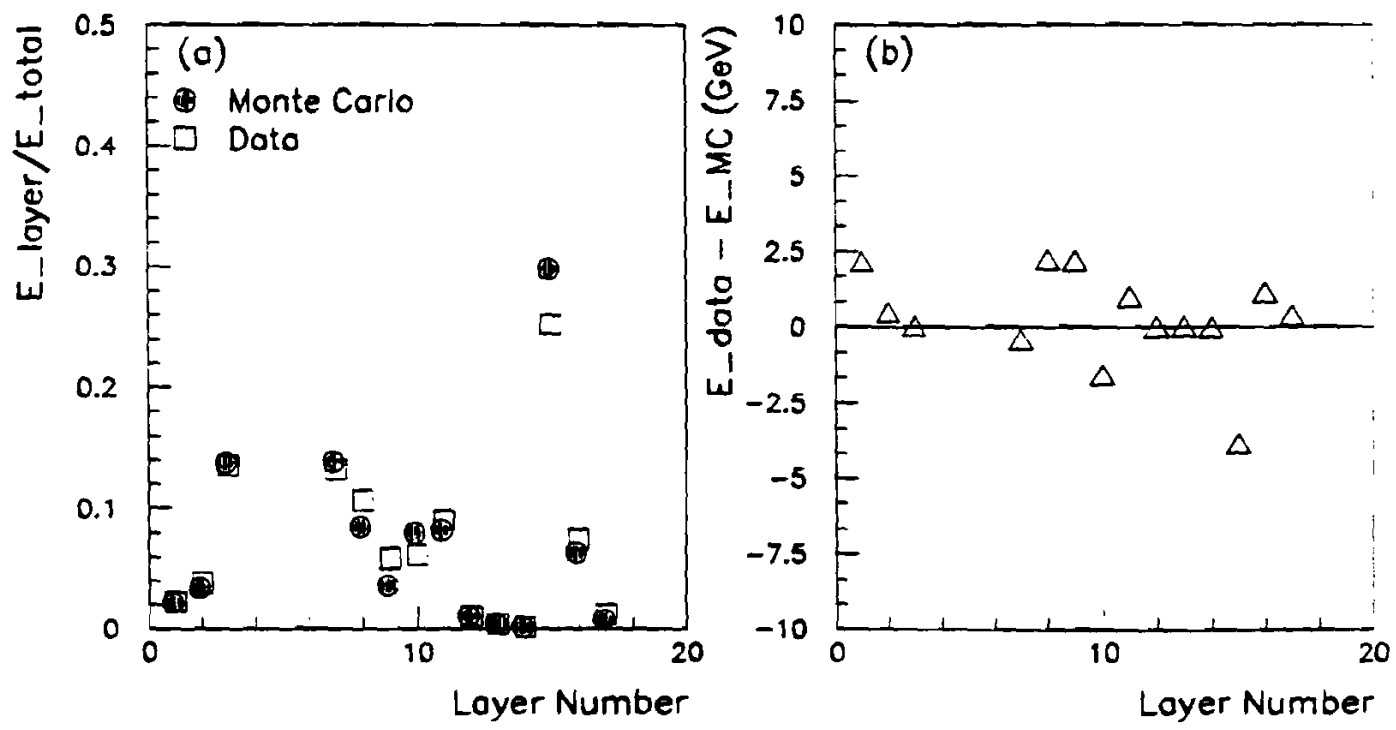

Figure 6.8: A comparison between test beam data with Monte Carlo simulation data at $\eta=1.05$. (a) shows the fractional energy in each readout layer for data and simulation. (b) shows the difference between the energy in each layer for the Monte Carlo simulation data and the real data. 
Fractional Energy Comparison, $100 \mathrm{GeV} \pi, \eta=1.15$
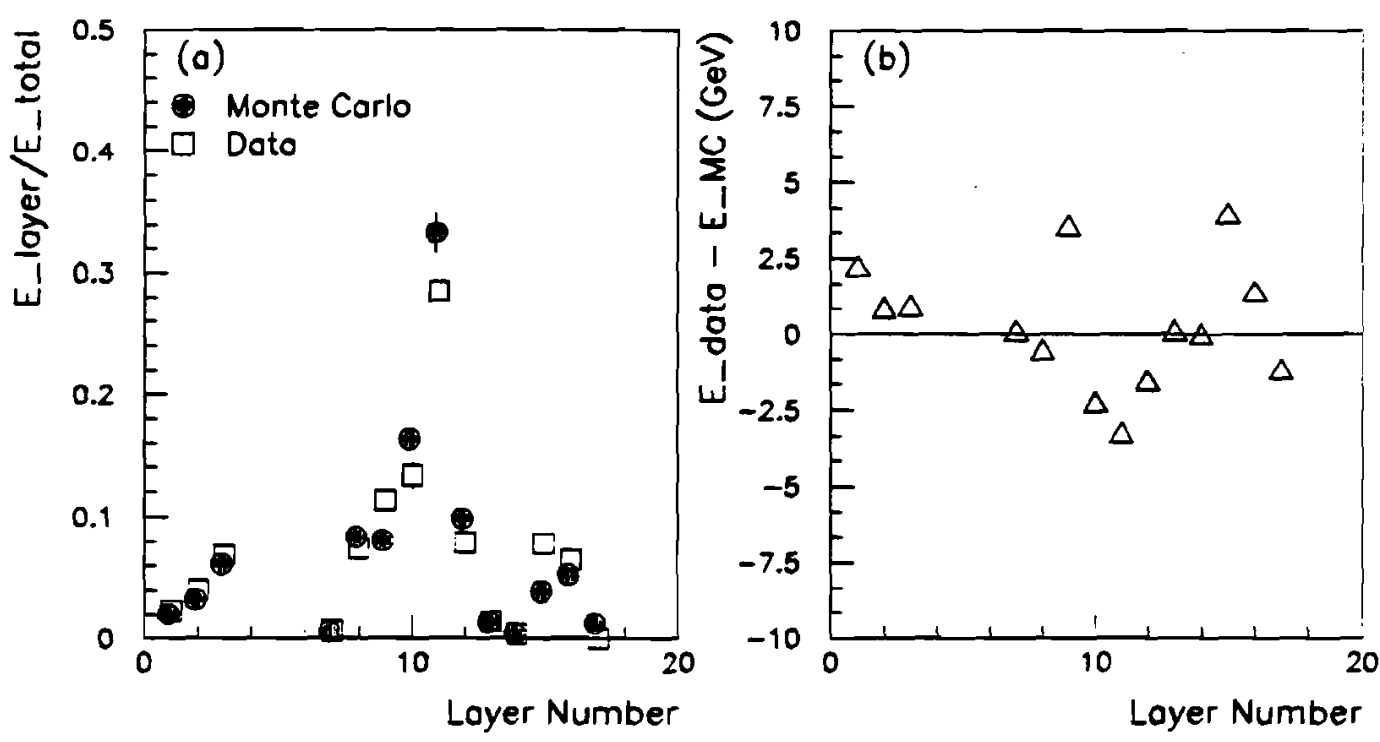

Figure 6.9: A comparison between test beam data with Monte Carlo simulation data at $\eta=1.15$. (a) shows the fractional energy in each readout layer for data and simulation. (b) shows the difference between the energy in each layer for the Monte Carlo simulation data and the real data.

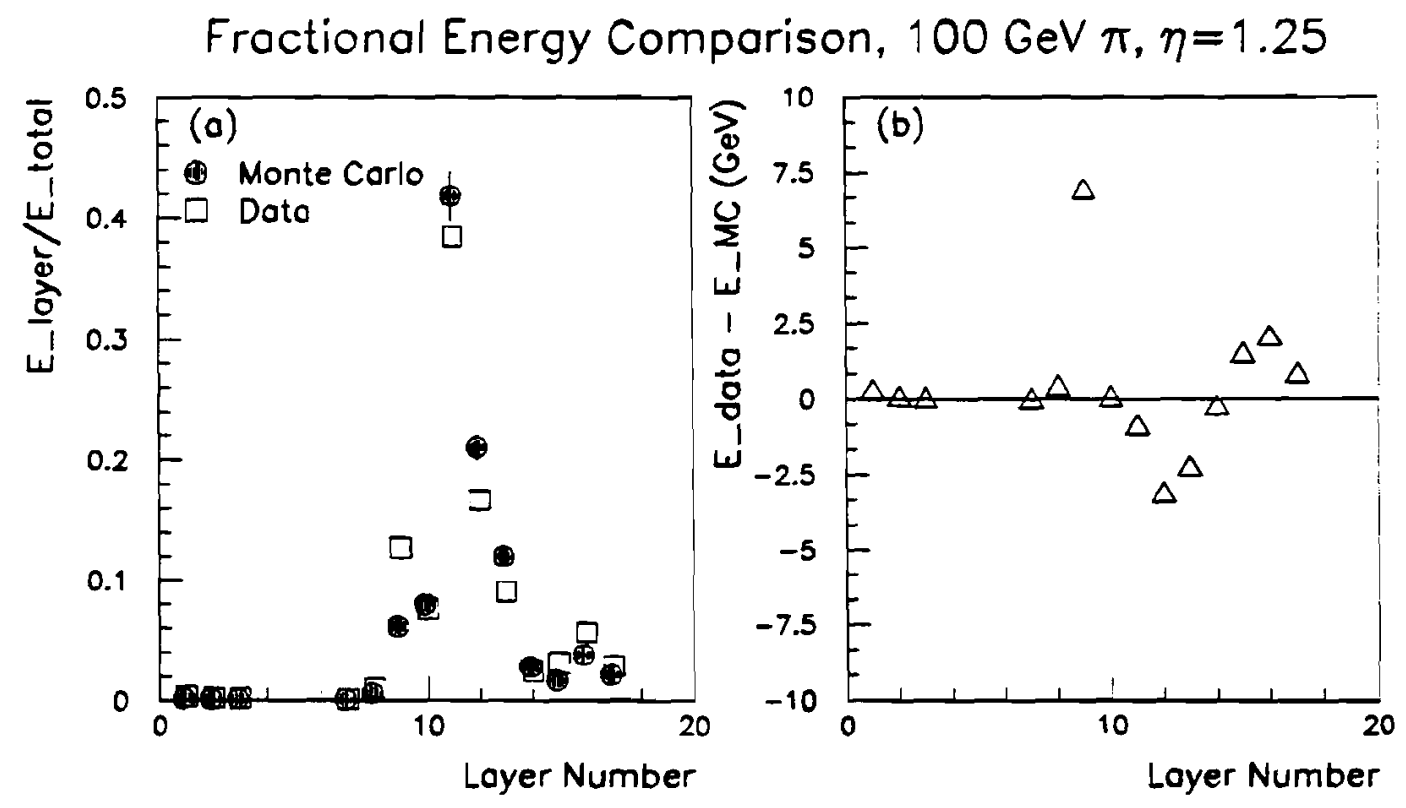

Figure 6.10: A comparison between test beam data with Monte Carlo simulation data at $\eta=1.25$. (a) shows the fractional energy in each readout layer for data and simulation. (b) shows the difference between the energy in each layer for the Monte Carlo simulation data and the real data. 
Fractional Energy Comparison, $100 \mathrm{GeV} \pi, \eta=1.35$
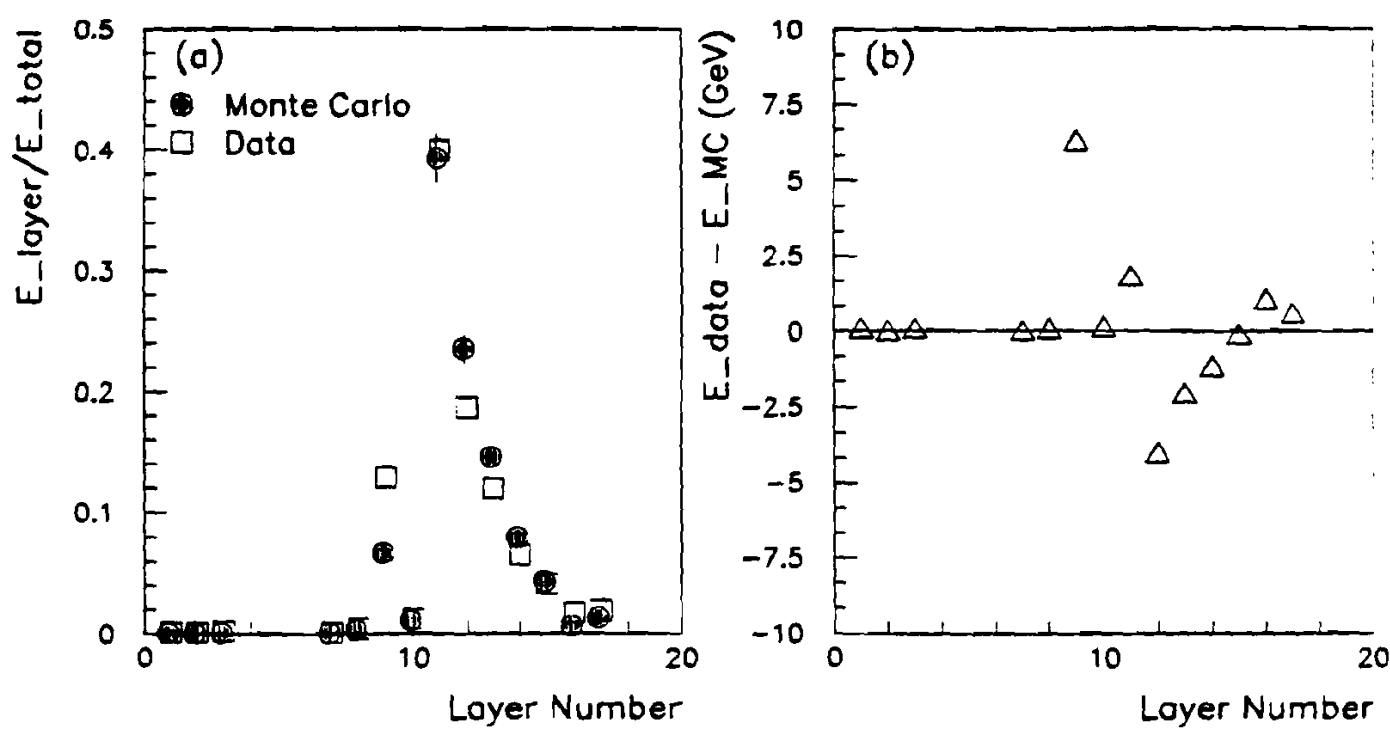

Figure 6.11: A comparison between test beam data with Monte Carlo simulation data at $\eta=01.35$. (a) shows the fractional energy in each readout layer for data and simulation. (b) shows the difference between the energy in each layer for the Monte Carlo simulation data and the real data. 

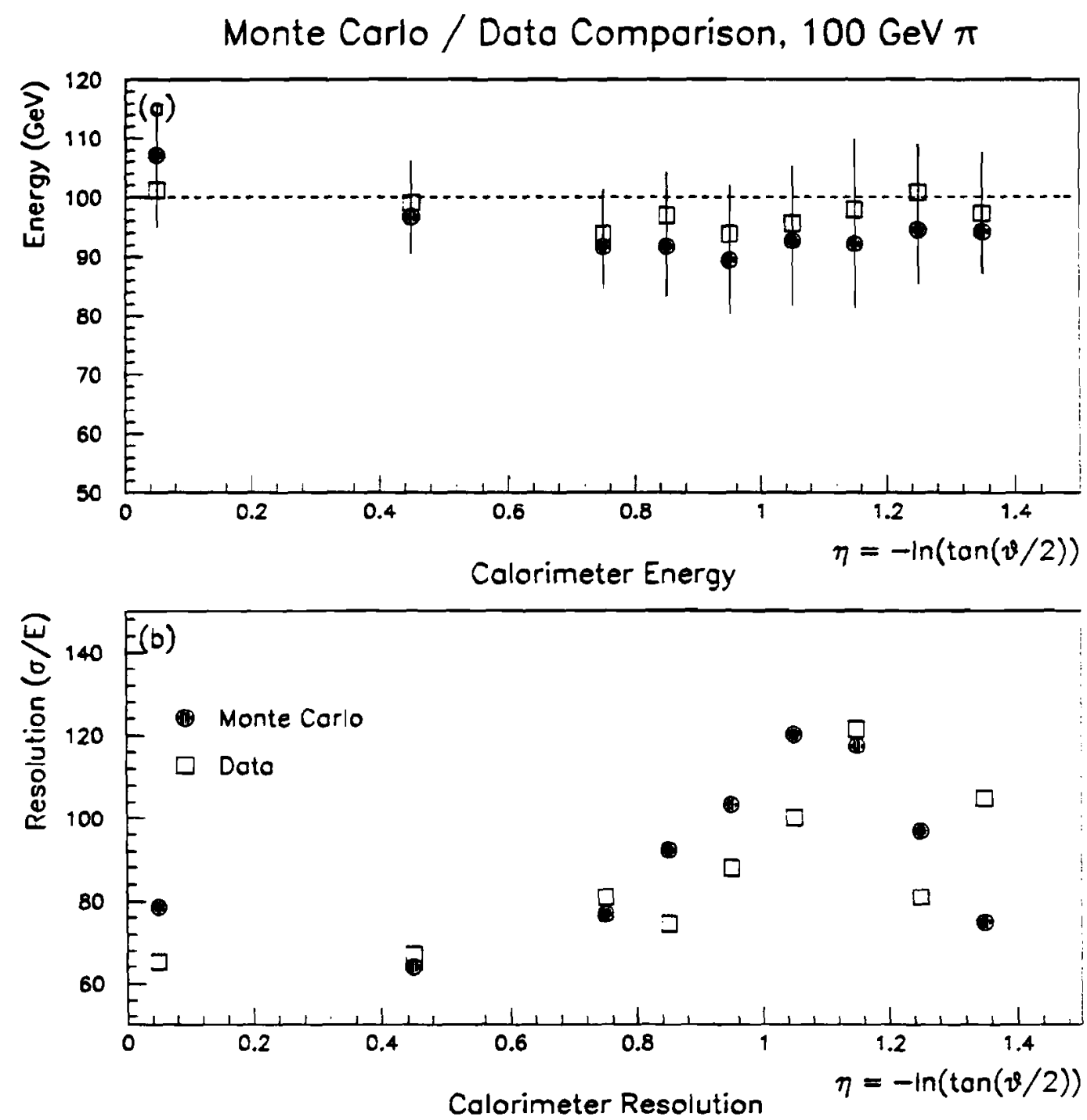

Figure 6.12: A comparison between test beam data and Monte Carlo simulation data for the (a) calorimeter total energy and (b) resolution. 


\section{CHAPTER 7}

\section{THE IMPACT OF THE INTERCRYOSTAT DETECTOR ON D $\emptyset$ PHYSICS}

A thesis such as this would not be complete without a brief look at how the ICD is performing in-situ in the $D \emptyset$ experiment. The $D \emptyset$ detector started taking data in May, 1992. To date, the experiment has received an integrated luminosity, $\int L d t$, of $\sim 28 \mathrm{pb}^{-1}$ and written $\sim 15 \mathrm{pb}^{-1}$ to tape. The integrated luminosity is plotted in Figure 7.1. Due to the main ring, $D \emptyset$ suffers from an increased dead time. A special veto has been implemented to block off the time when a proton store is being ejected into the main ring. This veto greatly decreases main ring background and accounts for approximately $22 \%$ of the dead time.

\subsection{Jets}

Event displays are a useful way to get an intuitive feel for collider events. The next set of figures, Figures 7.2 and 7.3 , are an event display from a two jet event, with both of the jets in the intercryostat region. Figure 7.2 is an $R-Z$ view (radial view, showing the $z$-axis, parallel to the beam pipe). The energy deposition is summed over $\phi$ with the small circle at the bottom left of the picture indicating the $\phi$ range (all $\phi$ in this picture) which is summed. In this view of the calorimeter, one can see the two beam jets along the beam pipe. The two hadron jets are also clearly visible. The first jet is mostly in the central calorimeter electromagnetic section while the second jet is mainly in the ICR and end calorimeter. The ICD display is indicated by the thin lines between the cryostats. In this view, it is clearly lit up in response to energy deposition. The rest of the detector is remarkably clean of any energy deposition. The view in Figure 7.3 is an $R-\phi$ projection of the same event. The 
two jets are clearly back-to-back. The top jet is mostly EM energy, while the bottom jet is almost entirely ICD and MG energy. The $\mathbb{Z}_{\mathrm{T}}$ is in the direction of the bottom jet. One can easily imagine the increase in the calculated $\mathbb{F}_{\mathrm{T}}$ if the intercryostat detectors were not available.

The excellent $\mathrm{D} \emptyset \mathrm{E}_{\mathrm{T}}$ resolution for minimum bias events is shown in Figure 7.4 as a function of the total $\not_{\mathrm{T}}[66]$. The $\mathbb{Z}_{\mathrm{T}}$ resolution is $\sigma\left(\mathbb{Z}_{\mathrm{T}}\right)=1.08 \mathrm{GeV}+0.019 \sum \mathbb{T}_{\mathrm{T}}$. The CDF Collaboration, also running at FNAL, finds the $\boldsymbol{Z}_{\mathrm{T}}$ resolution of their detector to be $\left(0.47 \pm 0.03 \mathrm{GeV}^{1 / 2}\right)\left[\sum E_{\mathrm{T}}\right]^{1 / 2}[67]$. For comparison, Table 7.1 lists the $\mathrm{D} \emptyset$ and CDF $\mathbb{E}_{\mathrm{T}}$ resolutions at various energies. $\mathrm{D} \emptyset$ compares favorably with the CDF $\mathbb{F}_{\mathrm{T}}$ resolution, indicative of its high degree of hermeticity.

\begin{tabular}{|c|c|c|}
\hline Energy $(\mathrm{GeV})$ & $\mathrm{D} \emptyset \sigma\left(\mathbb{F}_{\mathrm{T}}\right)(\mathrm{GeV})$ & $\mathrm{CDF} \sigma\left(\mathrm{F}_{\mathrm{T}}\right)(\mathrm{GeV})$ \\
\hline 50 & 2.0 & 3.3 \\
\hline 100 & 3.0 & 4.7 \\
\hline 200 & 4.9 & 6.7 \\
\hline
\end{tabular}

Table 7.1: A comparison of the $\mathbb{Z}_{\mathrm{T}}$ resolution at various energies for the $\mathrm{D} \emptyset$ and CDF detectors.

A preliminary calculation of the inclusive cross section for jet production, $d \sigma / d \mathrm{E}_{\mathrm{T}} d \eta$, is shown in Figure $7.5[68]$. The jets are restricted to the central region $(|\eta| \leq 0.9)$ and the errors include the systematic uncertainty in the energy scale of the $D \emptyset$ detector. A nextto-leading order theoretical calculation is plotted on top of the experimental distribution.

The impact of the intercryostat detectors can be seen by considering Figure 7.6 [69], which shows jet production as a function of jet $E_{T}$ (number of events for a particular $E_{T}$ ), for the full calorimeter (solid points) and for the calorimeter without the ICD and MG's (dashed points). As can be seen, including the intercryostat region detectors can have a significant effect on the measured $E_{T}$ of a jet. For instance, at an $E_{T}=40 \mathrm{GeV}$, without the intercryostat region detectors, the number of jets is mismeasured by nearly a factor of two. This type of mismeasurement would severely degrade the ability of $D \emptyset$ to do basic QCD studies such as the inclusive cross section for jets as shown above. 


\subsection{Jet-Photon Asymmetry}

A striking example of the impact of the intercryostat detectors can be seen by considering events with a single jet and photon [69]. Such an event is shown in Figure 7.7. The single photon candidate is clearly seen in bottom part of the CCEM, with no central detector tracks pointing to it (i.e., not an electron). The balancing jet can be seen in the EC. Events such as this can be used, for example, to compare detector performance in the CC and EC. The jet energy should balance the photon energy. We define the asymmetry variable as:

$$
A=\frac{E_{\mathrm{T}}^{J e t}-E_{\mathrm{T}}^{\gamma}}{0.5\left(\mathrm{E}_{\mathrm{T}}^{J^{e t}}+\mathrm{E}_{\mathrm{T}}^{\gamma}\right)} .
$$

This variable is a measure of the fractional missing energy of the jet. Figure 7.8 shows the asymmetry as a function of jet eta where the photon has been restricted to the central calorimeter and the jet can be anywhere. Figure 7.8(a) plots the transverse energy of the photon and the jet. The events have been selected by two different triggers. The first peak in the photon $\mathrm{E}_{\mathrm{T}}$ spectrum is from an $\mathrm{E}_{\gamma} \geq 12.0 \mathrm{GeV}$ Level 2 trigger; the second peak is from an $E_{\gamma} \geq 30.0 \mathrm{GeV}$ trigger. The energy of the jet has not been selected.

The asymmetry is then plotted in Figure 7.8(b) without the ICD and massless gaps and (c) with the ICD and MG's. Including the information from the intercryostat region detectors, the energy of the balancing jet is clearly better measured. Without these detectors, the energy of the balancing jet can be mismeasured by as much as $40 \%$. The general negative shift of the asymmetry is due to several different effects. First, the jet is defined by a cone algorithm, i.e., all energy within a cone defined by $\left[\Delta \eta^{2}+\Delta \phi^{2}\right]^{1 / 2}=0.7$ is summed and used as the 'total energy' of the jet. However, there is always leakage outside the cone and that energy is lost. Hence, the energy of the jet will always be lower than the energy of the photon. In addition, there are kinematic effects. In Figure 7.8(a), the structure of the photon energy spectrum is due to the trigger requirement that the energy of the photon is greater than a certain energy (12 or $30 \mathrm{GeV}$ ). However, the distribution of the jet energies is somewhat Gaussian around the peaks of the photon distribution. Thus, while there will be $15 \mathrm{GeV}$ photons, for instance, that are balanced by $11 \mathrm{GeV}$ jets, the reverse will not be true and there is a general shift towards negative asymmetry. The most important point, though, is that this asymmetry study is one of the strongest evidences of the impact of the intercryostat region detectors on the performance of the $D \emptyset$ detector.

Jet balancing or jet-photon balancing methods such as this were once considered as 
possible methods of calibrating the intercryostat region detectors, before our proposal to include the ICD in the test beam run. Looking at Figure 7.8(c), there are still fluctuations in the asymmetry variable. These fluctuations are partly due to statistics and partly due to other incompletely understood effects, such as a shift in the vertex or cone size effects. While the improvements in the asymmetry using the ICD and MG's are obvious, it is not obvious that we could have calibrated the detectors starting only from such information as in plot (b). The ICD and MG calibration constants gleaned from the test beam run were crucial to DØ's ability to fully utilize the calorimeter immediately upon the startup of the collider run.

\subsection{Top Search}

Since the beginning of the run, the $D \emptyset$ top analysis group has been hard at work on searches for the top. The most promising decay channel, due to the low background, is the $t \bar{t} \rightarrow W W b \bar{b} \rightarrow l_{\bar{l}}+\mathbb{Z}_{\mathrm{T}}+j$ jets and has been the focus of the initial search. While no definitive answers have yet come out of that analysis, $D \emptyset$ has interesting events which are not inconsistent with the hypothesis of a leptonic top decay. One such event is shown in Figure 7.9 and Figure 7.10. In the first figure, a restricted $\phi$ view, the $\mu$ can be seen going through the three layers of muon chambers and electron can be seen in the lower part of the CCEM. An exploded view of the calorimeter showing all $\phi$ is seen in Figure 7.10. The $\mu$ leaves a minimum ionizing trail in the top half of the calorimeter, the two jets can be seen crossing from the $\mathrm{CC}$, through the intercryostat region and ICD and into the EC. 


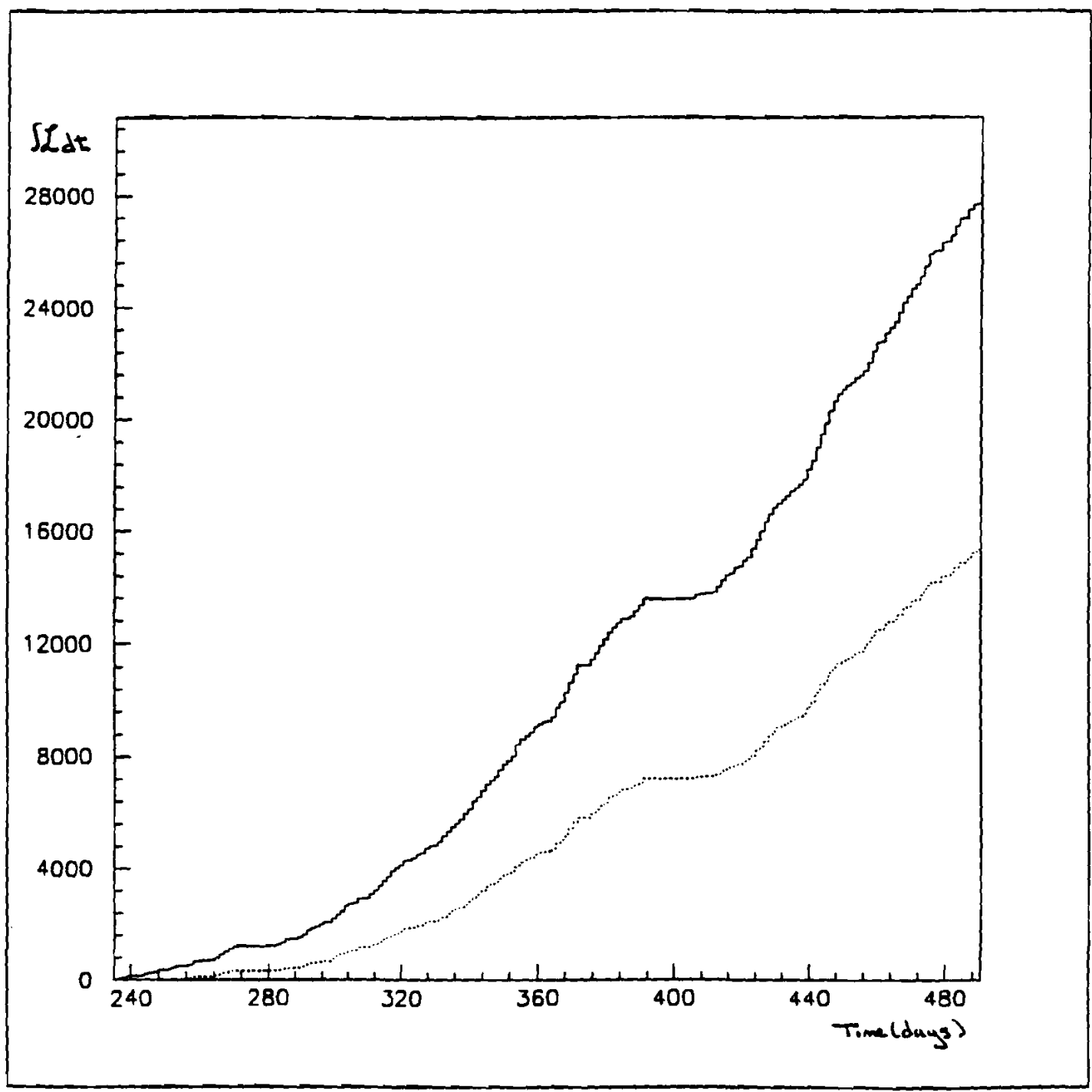

Figure 7.1: The DO integrated luminosity is plotted as a function of the number of days since accelerator start. The solid curve is the delivered luminosity; the dotted curve is the luminosity written to tape. 


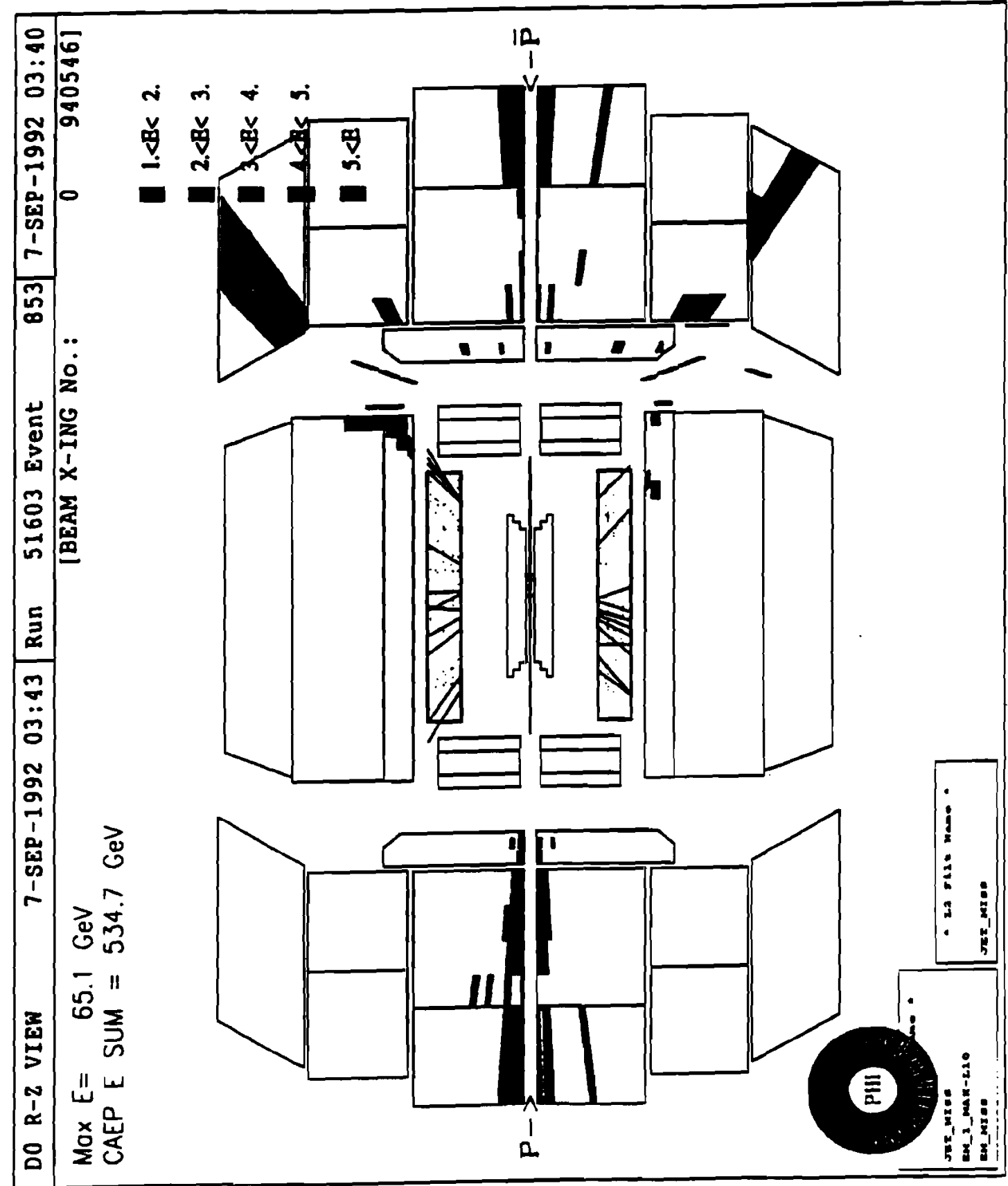

Figure 7.2: An event display from a two jet event, with both jets depositing energy in the intercryostat region. This is the $R-Z$ view. 


\section{$D \varnothing$ preliminary}

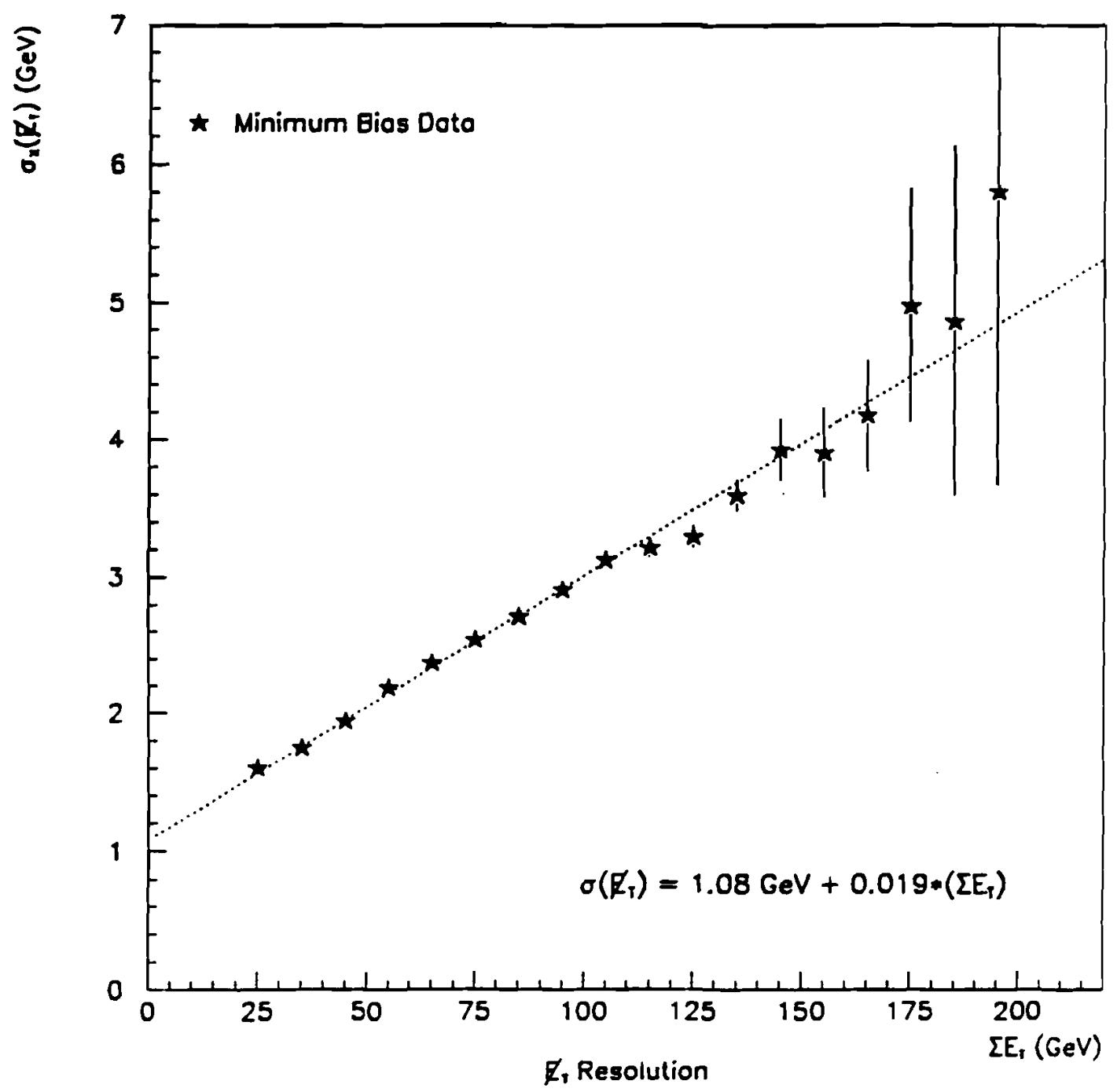

Figure 7.4: The $\Psi_{T}$ resolution of the $D \emptyset$ detector as a function of total \# $_{\mathrm{T}}$ for minimum bias events. 


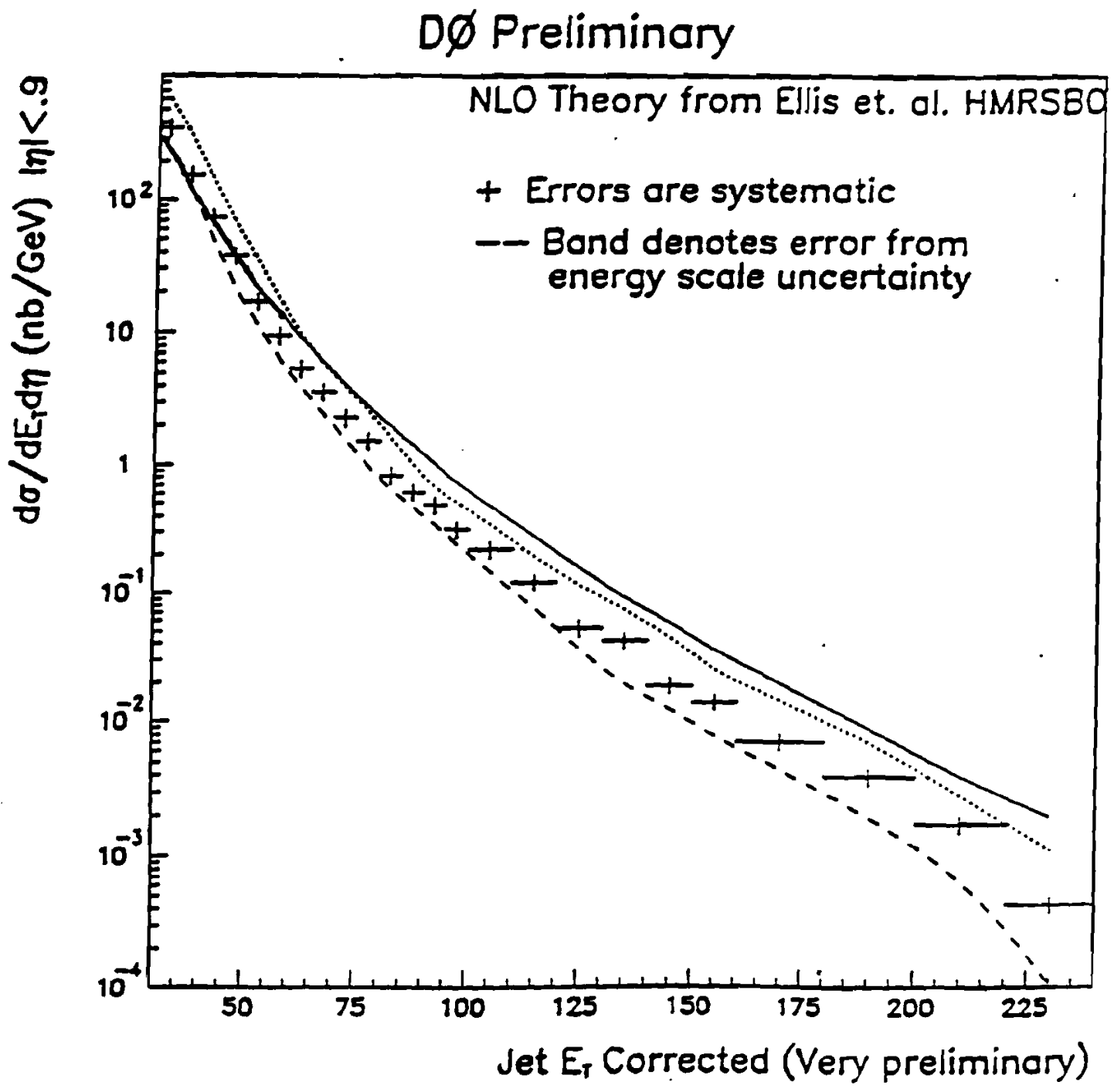

Figure 7.5: The inclusive cross section for jet production, $d \sigma / d \mathrm{E}_{\mathrm{T}} d \eta$, as a function of $\mathrm{E}_{\mathrm{T}}$ of the jet. A next-to-leading order calculation is also plotted for comparison. 


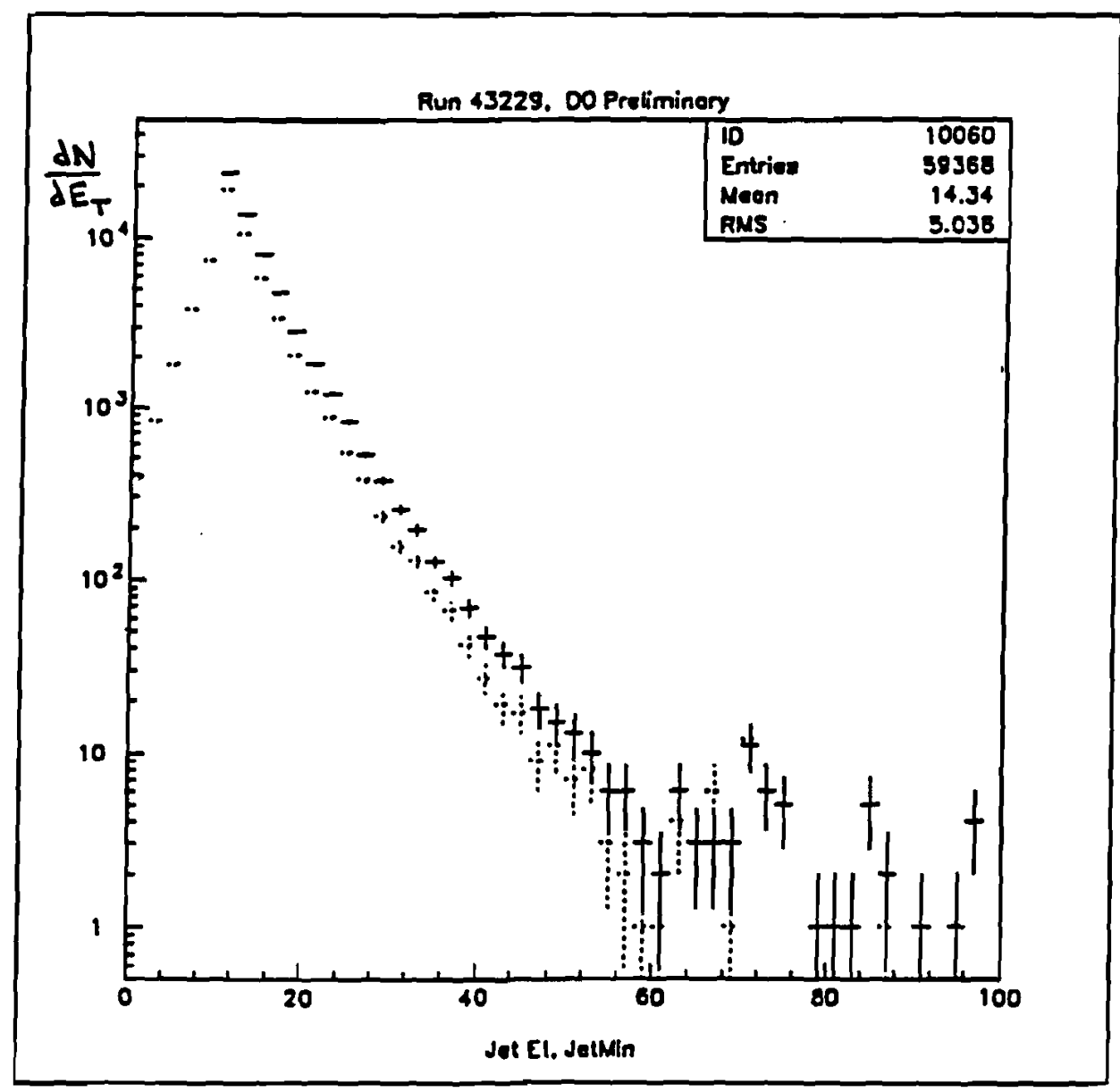

Figure 7.6: Jet production, $d N / d E_{T}$, as a function of jet $E_{T}$, for events which have at least one $\geq 20 \mathrm{GeV}$ jet. Using the full calorimeter yields the solid points, using the calorimeter without the information from the intercryostat detectors yields the dashed points. Error bars are statistical only. 


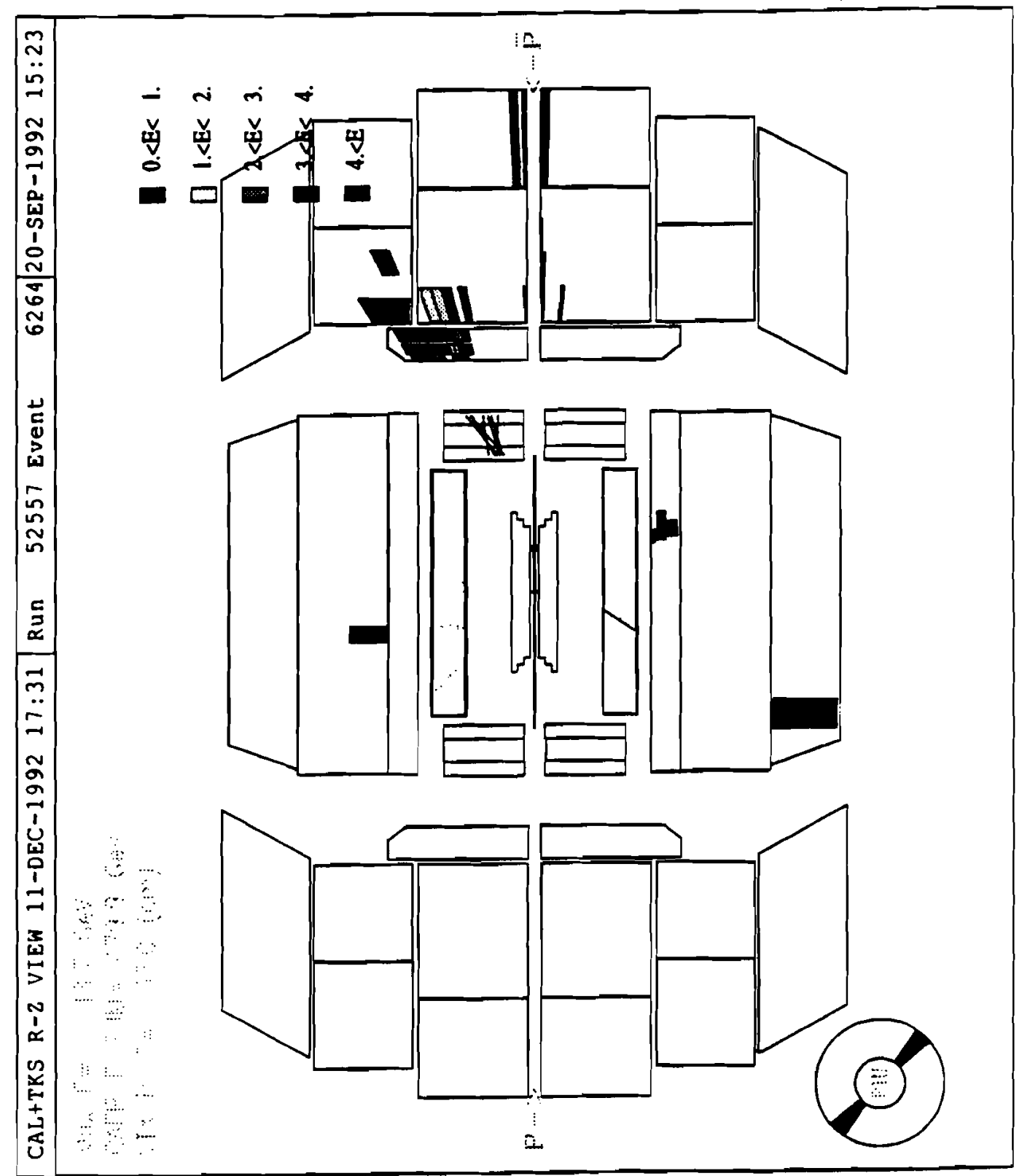

Figure 7.7: A calorimeter view of a direct photon candidate event. 

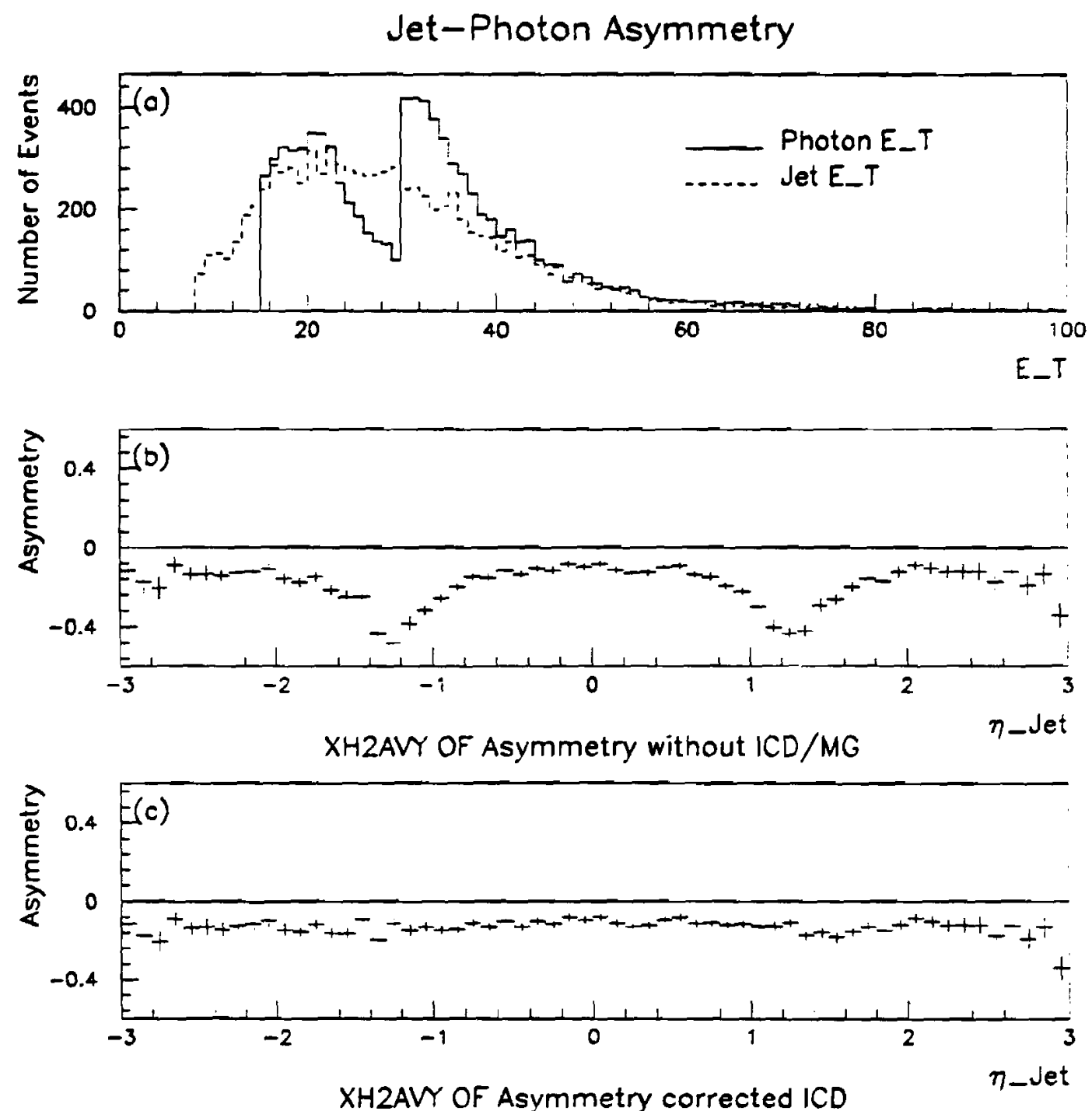

Figure 7.8: The asymmetry $\left(A=\frac{E_{T}^{J n}-E_{T}^{\gamma}}{0.5\left(E_{T}^{j e t}+E_{T}^{\gamma}\right)}\right)$ is plotted for events with a single photon and a single jet. Plot (a) shows the photon and jet $\mathrm{E}_{\mathrm{T}},(\mathrm{b})$ plots $A$ as a function of the jet $\eta$ without the intercryostat region detector and (c) with the intercryostat region detectors. 


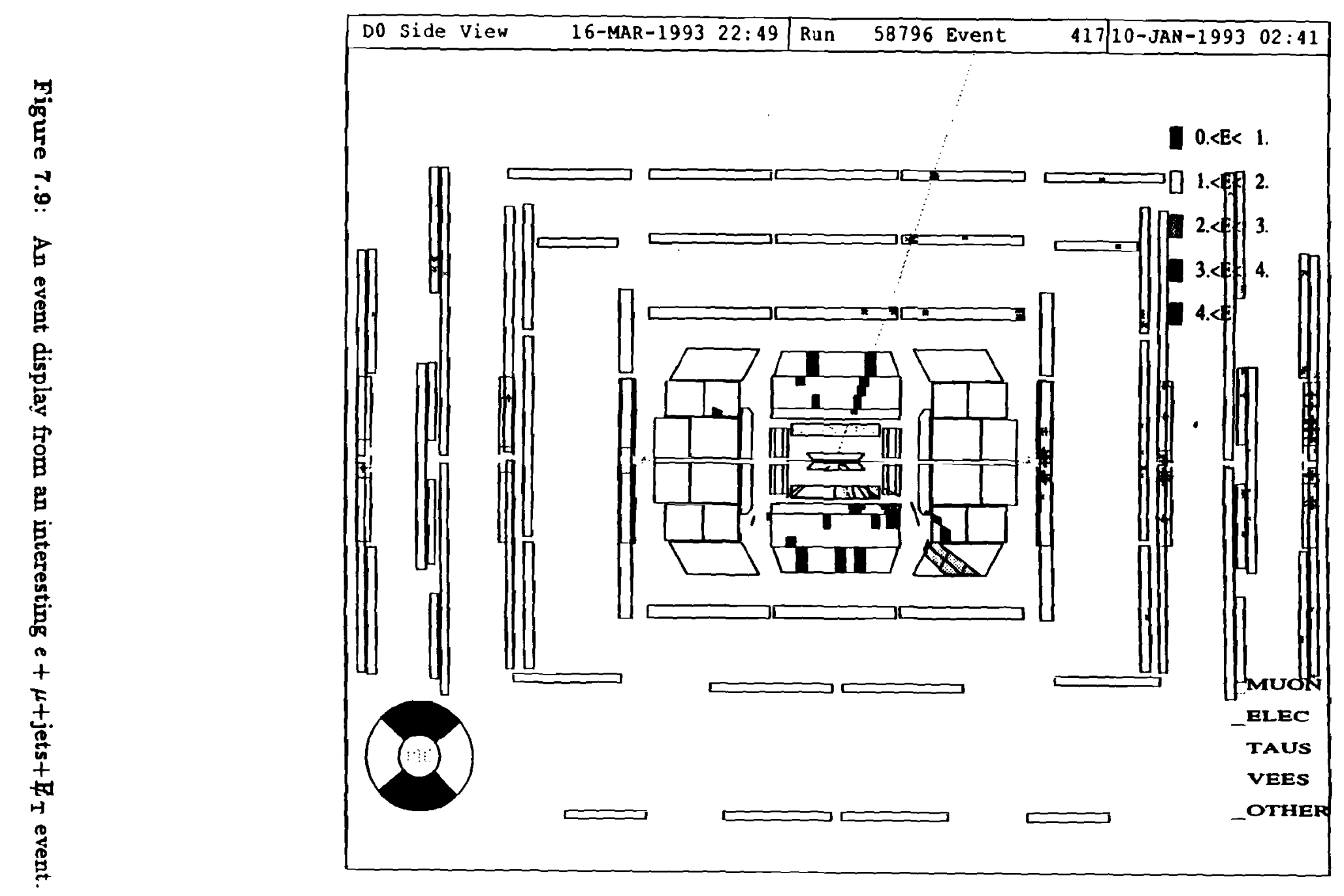



CHAPTER 8

\section{CONCLUSION}

We have designed and built a unique detector, the Intercryostat Detector. We have studied the response of that detector in the full $D \emptyset$ configuration using a test beam. The inclusion of the ICD into that test beam represented the first use of a scintillator sampling detector in a liquid argon environment. From the test beam project, we have determined the calibration constants (sampling weights) necessary to use the ICD in situ at $D \emptyset$ and additionally, have determined the sampling weights for the massless gaps. We have shown that the use of the ICD and the Massless Gaps dramatically improves the hermeticity and performance of the DO calorimeter. We have shorn that the ICD and MG's can make an important contribution to the physics program at $D \emptyset$. 


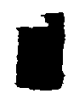

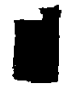

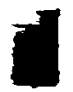

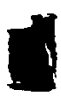

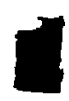

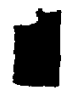

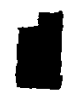

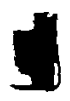

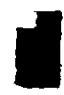

,

APPENDICES

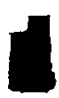

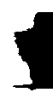

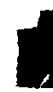

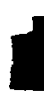

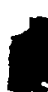

,

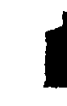

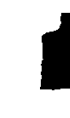

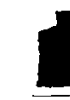


APPENDIX A

\section{The D $\emptyset$ Collaboration}

B. Gomez, B. Hoeneisen, D. Mendoza, P. Nechev, J.P. Negret, O. Ramirez, J. Roldan, A. Serna Universidad de Los Andes, Bogota, Columbia

J. Chen, D. Fein, G. Forden, E. James, K. Johns, P. Loch, L. Markovsky, B. May, A. Milder, J. Rutherfoord, M. Shupe, A. Smith, C. Zeitnitz

University of Arizona

S. Aronson, M. Fatyga, J. Featherly, B. Gibbard, H. Gordon, N. Graf, J.M. Guida, W. Guryn, S. Kahn, S. Protopopescu, P. Yamin Brookhaven National Laboratory

J. Bantly, D. Cullen-Vidal, D. Cutts, J. Hoftun, R. Lanou, F. Nang, D. Nešić, R. Partridge, H.W. Xu, R. Zeller Brown University

K. Bazizi, B. Choudhary, J. Ellison, R. Hall, A. Heinson, T. Huehn, A. Khachatouran, A. Kernan, S. Wimpenny University of California, Riverside

G. Alves, G. Lima, A.K.A. Maciel, V. Oguri, A. Santoro, M. Souza, M. Vaz Centro Brasileiro de Pesquisas Fisicas, Rio de Janeiro, Brazil

H. Casitlla, G. Herrera

CINVESTAV, Mexico City, Mexico

I. Adam, P. Franzini, U. Heintz, S. Kanekal, P.M. Tuts

Columbia University

V. Kapoor, R.K. Shivpuri

Delhi University, Delhi, India

S. Abachi, S. Ahn, J.F. Bartlett, P. Bhat, F. Borcherding, A. Brandt, A. Bross, J.M. Butler, J. Christenson, W.E. Cooper, M. Demarteau, N. Derisenko, H.T. Diehl, M. Diesburg, R. Dixon, D. Eartly, D. Elvira, H.E. Fisk, S. Fuess, C. Gao, K. Genser, C. Gerber, D.R. Green, H. Greenlee, H. Haggerty, S. Igarashi, A.S. Ito, M. Johnson, 
A. Jonckheere, H. Jostlein, B. Klima, S. Krzywdzinski, R. Li, Q.Z. Li-Demarteau, R. Lipton, L. Lueking, E. Malamud, I. Manning, H.-S. Mao, M.I. Martin, H. Melanson,

X.-C. Meng, W. Merritt, S. Mishra, H.E. Montgomery, C.T. Murphy, M. Narain,

N. Oshima, J. Ortmanns, A. Para, C.H. Park, A. Peryshkin, H. Prosper, Y. Que,

P. Quintas, P. Rapidis, R. Raja, A.L. Read, W. Smart, R.P. Smith, A. Taketani,

M. Tartaglia, D.-C. Wang, L. Wang, D. Wood, P. Xie, R. Yamada, M.-J. Yang, Y. Zhou, Y. Zhu

Fermi National Accelerator Laboratory

W. Dharmaratna, M. Goforth, S. Hagopian, V. Hagopian, A. Klatchko, S. Linn, R. Madden, H. Piekarz, C. Shaffer, H. Wahl, D. Xiao, S. Youssef Florida State University

J. Balderston, R. Cence, M. Cummings, M. Jones, M. Peters, C. Yoshikawa University of Hawaii

M. Adams, D. Averill, H. Goldberg, S. Margulies, T. McKibben, J. Solomon University of Illinois, Chicago

G. Alvarez, T. Marshall, H.J. Martin, C. Murphy, D. Zieminska, A. Zieminski Indiana University

E.W. Anderson, J.M. Hauptman, M. Pang, A. Zinchenko Iowa State University

J.S. Kang, C.L. Kim, D.W. Kim, S.K. Kim, H.K. Park, Y.M. Park Korea University, Seoul, Korea

H. Aihara, J. Bendich, L.P. Chen, A.R. Clark, O.I. Dahl, A. Goldschmidt, P. Grudberg, L. Kerth, S.C. Loken, R.J. Madaras, E. Oltman, N.A. Roe, A. Spadafora, M.L. Stevenson, M. Strovink, T. Trippe, P. Virador Lawrence Berkeley Laboratory

A. Baden, W.G. Cobau, N.J. Hadley, S. Kunori, A.L. Lyon, D. Norman, K. Streets, P. Tamburello University of Maryland

N. Amos, T.L. Geld, H.A. Neal, L. Oesch, J. Qian, D. Stewart, G.R. Snow University of Michigan

M. Abolins, R. Brock, D. Edmunds, S. Fahey, E. Flattum, K. Frame, R. Genik, N. Grossman, S. Jerger, J. Linnemann, J. McKinley, P. Mooney, D. Owen, B. Pi, B. Pope, H. Weerts, Y. Xia Michigan State University

P. Ermolov, A. Leflat, S. Rusin, E. Shabbalina Moscow State University, Russia

J. Kourlas, A. Mincer, M. Mudan, P. Nemethy, J. Sculli, J. Yang, Q. Zhu New York University 
R. Demina, M. Glaubman, S. Reucroft, T. Yasuda Northeastern University

M. Fortner, J. Green, D. Hedin, R. Markelof, V. Sirotenko, S. Willis Northern Illinois University

R.E. Avery, S. Blessing, D. Buchhoiz, B. Gobbi, Y.-K. Li, Y.-C. Liu, T. Joffe-Minor, H. Schellman Northwestern University

V. Balarmurali, N. Biswas, J. Jaques, R. Kehoe, M. Kelly, R. Ruchti, J. Warchol, M. Wayne University of Notre Dame

S. Beri, V. Bhatnagar, S. Chopra, J.M. Kohli, J.B. Singh, P.M. Sood Panjab University, Chandigarh, India

Y. Antipov, B. Baldin, V. Bezzubov, N. Bozko, V. Burtovoy, S. Chekulaev, D. Denisov, S. Denisov, A. Efimov, O. Eroshin, V. Evdokimov, A. Kirunin, B. Klochkov, V. Klyukhin, I. Kotov, V. Kochetkov, E. Kozlovsky, A. Mayorov, Y. Pischalnikov, V. Podstravkov, V. Riadovikov, A. Shkurenkov, D. Stoyanova, A. Suhanov, A. Vorobiev Institute for High Energy Physics, Protvino, Russia

B. Abbott, D. Koltick, R. McIlwain Purdue University

D. Adams, G. Eppley, H. Miettinen, J. Skeens Rice University

G. Blazey, J.P. Borders, C. Cretsinger, S. Durston, G. Fanourakis, T. Ferbel, S. Grudenendahl, R. Hirosky, K. Hodel, F. Lobkowicz, P. Slattery University of Rochester

Y. Ducros, J. Glicenstein, J.R. Hubbard, P. Mangeot, B. Mansoulie, A. Pluquet, J. Teiger, A. Zylberstejn CEN Saclay, France

R. Astur, D. Chakraborty, W. Chen, D. Claes, J. Cochran, R. Engelmann, S. Feher, G. Finocchiaro, M.L. Good, P. Grannis, J.A. Guida, T.C. Heuring, J. Jiang, C.K. Jung, C. Klopfenstein, H.L. Li; S. Lami, G. Landsberg, J, Lee-Franzini, S. Lökös, M. Marx, R. McCarthy, K. Ng, M. Parterno, D. Pizzuto, S. Rajagopalan, L. Rasmussen, M. Rijssenbeek, P. Rubinov, R.D. Schamberger, S. Snyder, J. Thompson, C. Yanigasawa, J. Yu State University of New York, Stony Brook

M. Botlo, L. Cormell, P. Dingus, M. Diwan, H. Fenker, S. Fredricksen, V. Glebov, H. Johnstad, J. Kotcher, K. McFarlane, C. Milner, T. Regan, D. Schmid, I. Sheer, F. Stocker, M. Takashima, E. Wang, J. Womersley Superconducting Supercollider Laboratory 
B.S. Acharya, M.R. Krishnaswamy, N.K. Mondal, V.S. Narasimham, M.V.S. Rao, P.R. Vishwanath

Tata Institute of Fundamental Research, Bombay, India

K. De, P. Draper, E. Gallas, J. Li, L. Sawyer, M. Sosebee, A. White University of Texas, Arlington

A. Boehnlein, M. Gui, R. Huson, J.T. White, J. Wightman

Texas A\&M University 
APPENDIX B

Study of inclusive $\Lambda$ production in $e^{+} e^{-}$annihilations at $29 \mathrm{GeV}$ 
Study of inclusive $\Lambda$ production in $e^{+} e^{-}$annihilations at $29 \mathrm{GeV}$

T. L. Geld, H. Neal, C. Akeriof, J. Chapman, D. Errede, (") M. T. Ken, P. Kesten, ${ }^{(b)}$ D. 1. Meyer, D. Nitz, R. Thun, and R. Tschirhart ${ }^{(e)}$ Unibersity of Michigen, Ann Arbor, Michigan 48109

S. Abachi, ${ }^{(d)}$ M. Derrick, P. Kooijman, ${ }^{(c)}$ B. Muagrave, L. Price, J. Repond, and $K$. Sugano Areonne National Laboratory, Argonne, Illinois 60439

D. Blocicus, "n B. B. Brabeon, J.-M. Brom, (s) C. Jung, (b) H. Ogren, D. R. Rust, and A. Snyder ${ }^{(i)}$

Indiane University, Bloomington, Indiane 47405

B. Cork

Lawrence Berkeley Laboratory, Berkeley, California 94720

P. Baringer, ${ }^{(j)}$ B. G. Bylsma, ${ }^{(k)}$ R. Debonte, E. H. Low, ${ }^{(1)}$ R. L. Mcllwain, D. H. Miller, C. R. Ng, K. Rangan, $(m)$ and E. Shibala

Pundue University, West Lafayetle, Indiana 47907

\title{
(HRS Collaboration
}

(Received 8 July 1991)

\begin{abstract}
Cross sections are presented for the inciusive production of $A$ byperons in electron-positron annihilations at $\sqrt{s}=29 \mathrm{GeV}$ based on the full 291-pb-1 sumple of dat taken in the High Resolution Spectrometer experiment at the SLAC $e^{+} e^{-}$stonge ring PEP. These results, and the associated correlation anslyses. are consistent with the Lund model predictions with the stnnge diquark suppression ratio $\delta$ fxed at $0.59 \pm 0.10 \pm 0.18$, as compared to the standard Lund value of 0.32 . The $A$ multiplicity has been found to be $0.182 \pm 0.020$ per event. The opposite-atrangeness multiplicity $\left\langle n_{A \pi}\right\rangle$ bes been mensured to be $0.046 \pm 0.020$, whereas the like-strangeness multiplicity $\left(n_{A A+\bar{A}}\right)$ is $0.009 \pm 0.028$. A strong correlation is found between $A$ 's and $\bar{\Lambda}$ 's; when one is found in an event, the other is found in the same event with a probability that exceeds $50 \%$.
\end{abstract}

PACS number(s): 13.65. $+i$, 13.87.Fh, 14.20Jn

(a) Present addrest: University of Illinois, Urbana. IL 61801.

tblPresent address: Santa Clara University. Santa Clara, CA 95053.

(c) Present address: Fermi National Laboratory, Batavis, IL 60510 .

(c) Present addreas: Fermi National Laboratory, Batavia, IL 60510.

(e) Present address: NIKHEF.H. Amsterdam. The Netherlends.

inpresent address: Staniord Linear Accelerator Center, Sten. ford. CA 94305.

(ePresent address: CRN Division de Hautes Energies, Strasbourg, France.

(b) Present address: Stanford Linear Accelerator Center, Stanford. CA 94305.

(i) Present address: Stanford Linear Accelerator Center. Stanford. CA 94305.

(j)Present address: University of Kansas, Lawrence, KA 66045. (k)Present addreas: Ohio State University, Columbus, $\mathrm{OH}$ 43210 .

'"'Present address: Virginia Polytechnic Institute and State University, Blacksburg, VA 24061.

Imi'Present address: Electronic Data Systerns of Canade. Oshawa, Canada.

\section{INTRODUCTION}

We report herein the results of an analysis of the inclusive production of $\Lambda$ hyperons in $e^{+} e^{-}$annihilations at $29 \mathrm{GeV}$ based on data from the High Resolution Spectrometer (HRS) experiment conducted at the SLAC $e^{+} e^{-}$stornge ring PEP. The $\Lambda$ cross sections presented are the first based on the final sample of HRS data. corresponding to an integrated luminosity of $291 \pm 7 \mathrm{pb}^{-1}$.

Details of the experimental apparatus have been previously published in Ref. [1], the techniques used to select $A$ 's have been summarized in Rer. [2] and previous results, based on an integrated luminosity of $256 \pm 8 \mathrm{pb}^{-1}$, have been reported in Ref. [3]. The present analysis, in addition to benefiting from the increased luminosity, is based on the standard HRS version of the Lund Monte Cario program $[4,5]$ (version 5.3), which is a more advanced version than previously used in any HRS inclusive $\Lambda$ analyzis. The previous analyses were based on detector acceptances calculated using Lund version 4.3 as the event generator. The new Monte Carlo sample also had twice as many $\Lambda$ 's as in the previous sample. In addition, the reconstruction techniques used previously varied from the "standard" HRS cuts summarized in 
Ref. [2]. The present analysis uses a more direct approach in determining the background in the single and dual particle spectra. The particular techniques employed in refining the data smples utilized are described below.

Studies of the inclusive production of neutral kaons and lambda hyperons are relevant to the general question of how strange particles are produced in $e^{+} e^{-}$collisions. The cross sections for these processes, when compared with those from other reactions, provide an insight into strange-quark fragmentution. Further, events contuining multiple strange particles can be used to determine what knowledge one strange particle has sbout the production of the others in the ame eveat. Whether or not strange particles with opposite strangeness are more likely to be in the ame jet than in opposite jets is an example of a question whose answer relates directly to the production process.

The problem under study, however, extends well beyond the tracking of strangeness quantum numbers. There is considerabie interest in the question of how baryons in general are produced in the fragmentation process. Though the subject has been explored by several experiments in recent years, numerous central issues remnin unresolved. The baryons mose acceasible for study are the proton and lambda hyperon. Complicating the analysis of the production of these particles, however. is the fact that they arise as products of $e^{+} e^{-}$annihilations both as a result of fragmentution processes and as a result of the decays of other baryonic states.

In present fragmentation models baryons are produced via the creation of diquark pain which then combine with a neighboring quark to produce the baryon. The Lund model parameter that governs the ratio of the baryonic to mesonic production rates is $(q q / q)$, specifying how much more dificult it is to produce 2 diquerk pair than a quark pair. In the inclusive production of $A$ 's the strangeness quanta can come either from the quark or the diquark. To describe both the $\boldsymbol{K}^{0}$ and $\Lambda$ inciusive production dats, two additional parameters are required: $(s / d)$ and $\delta=(u s / u d) /(s / d)$. The first parameter specifies the relative rate of the production of strange quarks and light quarks. The second specifies the extra suppression associated with the production of a strange diquark in contrast with the production of a diquark composed of light quarks - over and beyond what would be expected on the basis of the suppreasion due to $(s / d)$ alone.

A variant of this simple parametrization for produciag baryons is the "popcorn model," in which the remnant quarks left over after the quark pair and diquerk pair produce a baryon contribute to the production of intermediate mesons and other baryons, rather than directly forming the counterpart antibaryon. This effect is illustrated in Fig. 1 [6]. The simple Lund model implies a rather strong correlation between $\Lambda \bar{\Lambda}$ pairs, while the popcorn model implies a much weaker correlation between the two. Recent work by a UCLA group [7] attempts to avoid the heavy parametrization of the Lund model by relying on two minimal assumptions: the Wilson area law and the principle of hadronic phase space.
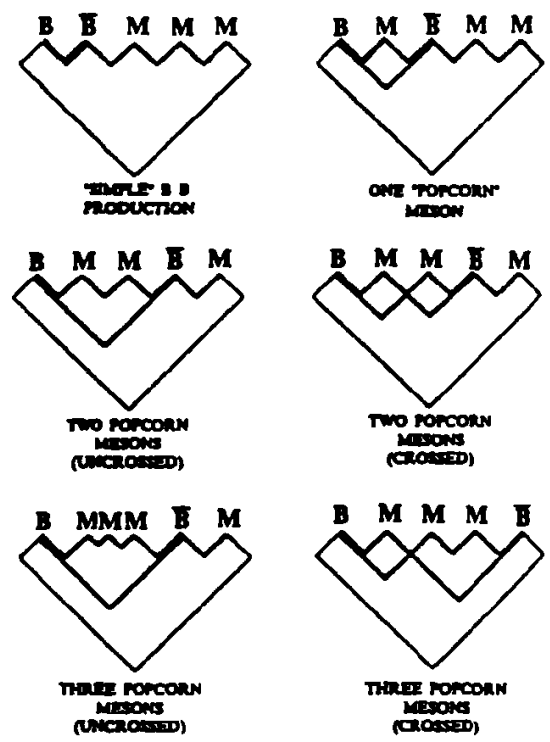

FIG. 1. Comparison of possible production mechanisms.

Their general overall agreement with available data is impressive and their $\Lambda^{0}$ multiplicity prediction of $0.18 \mathrm{com}$ pares well with the value to be presented here.

In this paper we utilize the large sample of $\Lambda$ and $\bar{\Lambda}$ events to extract the $\delta$ parameter and to examine the dominance of the simple Lund fragmentation mechanism through an analysis of the production rates of likestrangeness and opposite-strangeness $\Lambda(\bar{\Lambda})$ pairs.

The HRS consisted of an inner and outer driftchamber system which permitted charged-particle track positions to be determined to $200 \mu \mathrm{m}$ over a radial distance of $1.89 \mathrm{~m}$, and a barrel shower system which had an energy resolution of $\pm 0.16 / \sqrt{E} \mathrm{GeV}$, all contained within : 16-kG longitudinal magnetic feld. Because of the signature of vee events within the harge volume of the HRS detector, it was possible to identify $\Lambda$ hyperons with - high level of confidence. In the 123000 hadronic events observed in the experiment, there were 8698 neutral keons, $1259 \Lambda^{\prime}$ 's and $1124 \bar{\Lambda}$ 's. The $\Lambda(\bar{\Lambda})$ events represent the single largest sample of any experiment conducted in this energy region. An analysis of the neu. tral kaon events has been reported in a separate publication [B].

We summarize below the selection steps used to extract the final set of data utilized in this analysis, and describe the selection criteria used in the identification of lambdas. Also, the approuch used to determine the background for each mass bin is discuseed. Then a summary of the cross sections for lambda production is given as a function of $z$ the fractional energy, and $y$ the rapidity. Comparisons are made with previously published resuits and a summary is provided of the Lund model parameters resulting from fits to the cross section.

Correlations between $\Lambda$ 's and $\bar{\Lambda}$ 's in the same event are also reviewed in an effort to determine the extent to 
Which the production of multiple strange particles might be enhanced or retarded beyond that one would expect on the basis of the random single-particle production probabilities.

\section{EVENT SELECTION}

A typical event in the HRS bas 13 charged-particle tracks. To pick out a true vee in the midst of these tracks requires a rather sophisticated reconstruction process. The selection of the acturl data sample wes based on a set of conditions placed both upon the individual chrrgedparticle tracks and upon the track pairs [2]. The individual track conditions were the $\chi^{2}$ per degree of freedom from the fit of each track to the drift-chnmber hits had to be less than 10; the polar angle was constrained by the relation $\cos (\theta)<0.9$, where $\theta$ is the track angle with respect to the beam; the transverse momentum was restricted by the condition $p_{t}>120 \mathrm{MeV} / \mathrm{c}$, the track had to pass within $0.4 \mathrm{~m}$ of the intersection point in the $z$ direction (along the beam line), and within $0.3 \mathrm{~m}$ in the $x-y$ piane; at least $50 \%$ of the drift chamber layers traversed by the track must have been used in the fit; and, reconstructed track segments from looping churged tracks are removed.

The potential ves were identified by systematic pairing of oppositely charged tracks. Intersection points were then calculated as secondary vertices and subjected to the following criteria: the distance in the $x-y$ plane from the primary to secondary vertex was greater than $9 \mathrm{~mm}$; the distance in $z$ between the two tracks at the intersection point was leas than $30 \mathrm{~mm}$; the. impact parameter of the neutral particle momentum at the primary vertex was less than $5 \mathrm{~mm}$; the tangent of the angle between the vee's momentum direction and the line connecting the primary and secondary vertices was less than 0.05; the sum of distances of closest approach to the primary vertex of the two tracks in the $x-y$ plane was greater than $2 \mathrm{~mm}$; the tracks had to have no drift chamber hits between the primary and secondary vertices; each event bad to have a charged track multiplicity greater than 5; and, in order to remove nonhadronic (e.g., two-photon or beam gas) events, the total energy was required to be greater than $10 \mathrm{GeV} / c$.

\section{Cross sections}

To determine the inclusive cross section for the production of $\Lambda$ 's (where $\Lambda$ refers to both $\Lambda$ and $\bar{\Lambda}$ ) it is necessary to extract the real number of $\Lambda$ 's above background in each $z$ or $y$ bin and the detector acceptance for each $z$ or $y$ bin.

The HRS Lund Monte Carlo simulation (version 5.3) was used to caiculate the acceptance of the detector. Particles generated by the Monte Carlo simulation were passed through a detector simulation program and the particles that emerged from the simulated detector were then subjected to the same cuts as the real particles. An error on the efficiency was calculated using a binomial distribution of the number of reconstructed particles and a contribution due to systematic uncertainties in back-

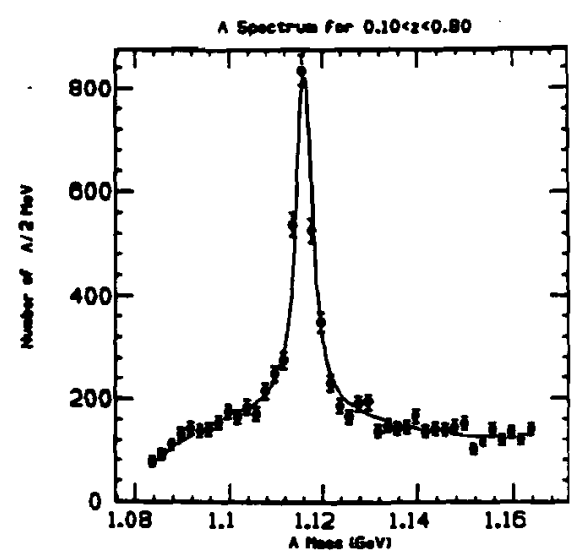

FIG. 2. Pion-proton mass distribution.

ground fit, which dominnted the error.

Figure 2 presents the pion-proton mass distribution, and a fit consisting of a Breit-Wigner (BW) and a thirddegree polynomial background. We find a $\Lambda$ central mass value of $1.1157 \pm 0.0001 \mathrm{GeV}$, and $\& \Gamma$ of $0.0042 \pm 0.0002$ GeV. In order to avoid the problem of having signal events in the tail of the $\Lambda$ Breit-Wigner be interpreted as background in a joine polynominl-BW forced fit, we made a separate determination of the background, removing all bins within $\pm 15 \mathrm{MeV}$ of the $A$ mass penk. The signal was then determined through a bin-by-bin subtraction of this background from the total bin populations.

The inclusive differential cross section for particle production is

$$
\frac{d \sigma}{d z}=\frac{N}{\Delta z A L} \text {. }
$$

where $N$ is the number of particles, $\Delta z$ is the width of the $z$ interval. $A$ is the acceptance of the detector in that $z$ in. terval, and $L=291 \pm 7 \mathrm{pb}^{-1}$ is the integrated luminosity. The cross section $s / B(d \sigma / d z)$ was calculated, using an adjusted value of $\sqrt{3}=28.3 \mathrm{GeV}$ to take into account ra. diative corrections.

The $\Lambda$ inclusive cross section is shown in Fig. 3, with the bin-by-bin values in Table I. The errors in the luminosity, the efficiency, the background fit, and the statistical error in the number of particies were added in quad-

TABLE I. Inclusive A cross sections as a function of fractional enersy.

\begin{tabular}{lcc}
\hline$z$ & No. of A & (s/B)do/dz (nb GeV $)$ \\
\hline 0.125 & 585.9 & $539.4 \pm 66.4$ \\
0.175 & 411.4 & $193.8 \pm 24.6$ \\
0.225 & 329.1 & $142.4 \pm 19.2$ \\
0.275 & 223.4 & $85.7 \pm 12.3$ \\
0.350 & 223.8 & $56.9 \pm 8.1$ \\
0.450 & 99.3 & $26.9 \pm 4.8$ \\
0.650 & 51.2 & $5.6 \pm 1.2$ \\
\hline \hline
\end{tabular}




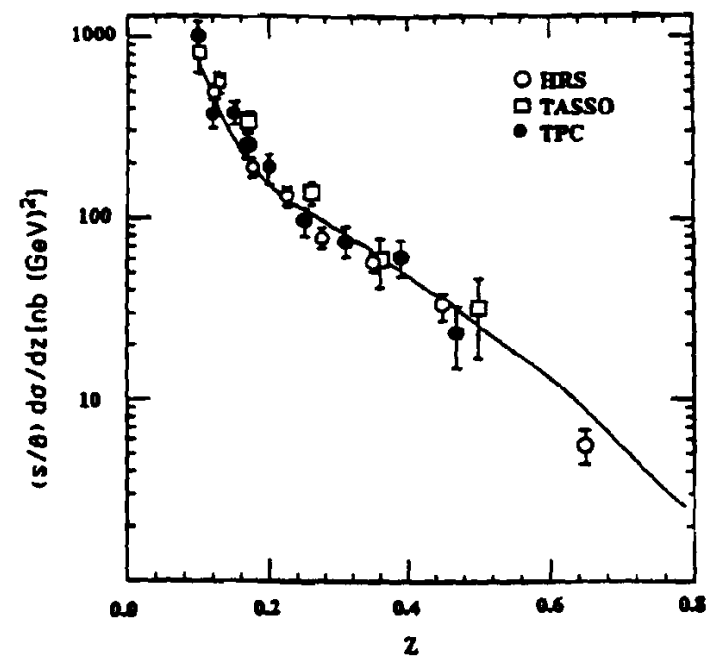

FIG. 3. Lambda-inclusive cross section at $29 \mathrm{GeV}$.

rature to get the final error quoted. Included for comparison are the results from other experiments in this energy range. As indicated, the agreement with the experiments of TASSO (at $34 \mathrm{GeV}$ ) [9] and TPC [10] is good.

The curve shown in Fig. 3 is a Lund fit, which is discussed later. Using these data we calculate tocal cross sections of $\sigma_{A}=52.8 \pm 6.0 \mathrm{pb}$ for the interval $0.10 \leq z \leq 0.80$. Extrapolating the Lund fit to the $\Lambda$ data to cover the unmeasured extremes gives a total cross section of $76.9 \pm 7.5 \mathrm{pb}$ for the interval $0.0 \leq z \leq 1.0$. These measurements lead to a single-particle multiplicity of $\left\langle n_{\Lambda}\right\rangle=0.182 \pm 0.020$.

As a check on the method we used to extract the $\Lambda$ signal above background, a similar analysis was made for the neutral kaon data. This analysis yieided $\sigma_{K^{0}}=472.5 \pm 9.7 \pm 11.4 \mathrm{pb}$ for the interval $0.06<z<0.70$. This value was then compared to the recentiy reported HRS value $\sigma_{x^{0}}=480.6 \pm 6.1 \pm 14.5 \mathrm{pb}$ for the same interval, determined independently using an analysis procedure designed to optimize the $K$ signal-tonoise ratio [8].

\section{LUND PARAMETERS}

The rate of production of inclusive lambdes in $e^{+} e^{-}$ annihilations can be parametrized in terms of the strengths of a small number of basic processes involved in the production of the strange quarks that end up in the final lambdas. In particular, the parameters that describe the rates for producing $s \bar{f}$ pairs $|s / u|$ and strange diquarks (us/ud] form the keys for specifying lambde production in the Lund model. From earlier analyses of the HRS data, the ratio of $K$ meson and pion inclusive cross sections yieids a $s / d$ value of $0.34 \pm 0.03$ [11]. The diquark ratio, $99 / q=0.078 \pm 0.005$, is from the TPC mea-

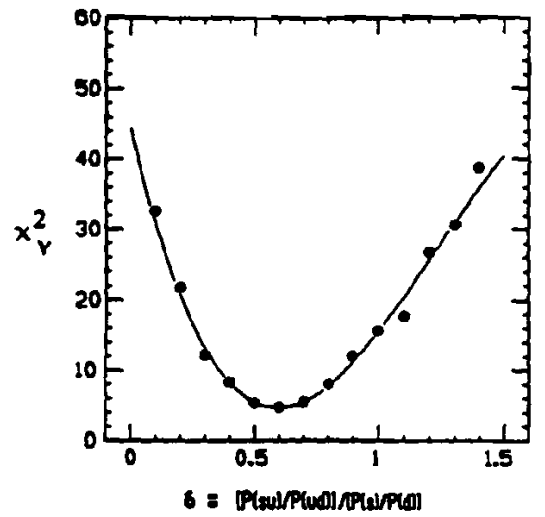

FIG. 4. $\chi^{2} v \delta . \chi^{2}$ is celculated by comparing the Lundgenerated $[(1 / \sigma) d \sigma / d z]$ distribution to the experimentally determined distribution.

surement of proton and pion inclusive cross sections [12]. We measure the final major parameter. $\delta=(u s / u d) /(s / d)$. There are other processes which can contribute to lambda inclusive production, including the decays of higher-mass strange particles, and the production of baryon-antiberyon systems with mesonic inter. mediate states, such as in the popcorn model. All known higher-mass strange baryon decays have been explicitly taken into account in our subsequent analysis. In particular, the charmed lambda hyperon decay $\Lambda_{c} \rightarrow \Lambda+X$ is explicitly taken into account with a branching ratio of $23 \% \pm 10 \%$ [13].

The sensitivity of the rapidity cross section to $\delta$ is illustrated in Fig. 4. At each point on the curve in Fig. 4, a $x^{2}$ was calculated by comparing the experimentally determined rapidity distribution (shown in Fig. 5) with the rapidity distribution predicted using the Lund Monte Carlo

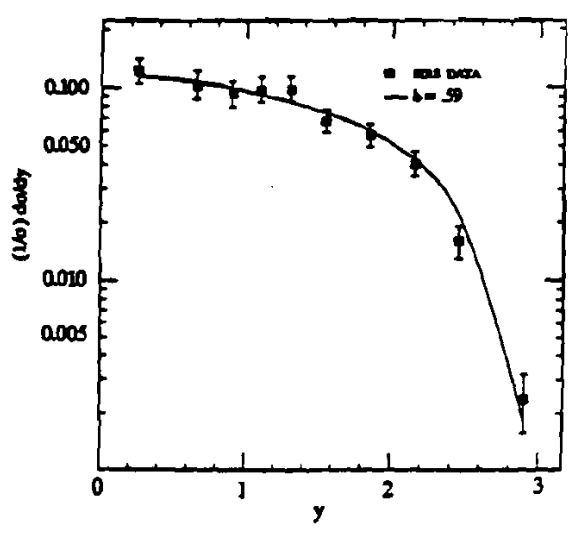

FIG. S. Lambda-inclusive cross section as a function of rapidity. 


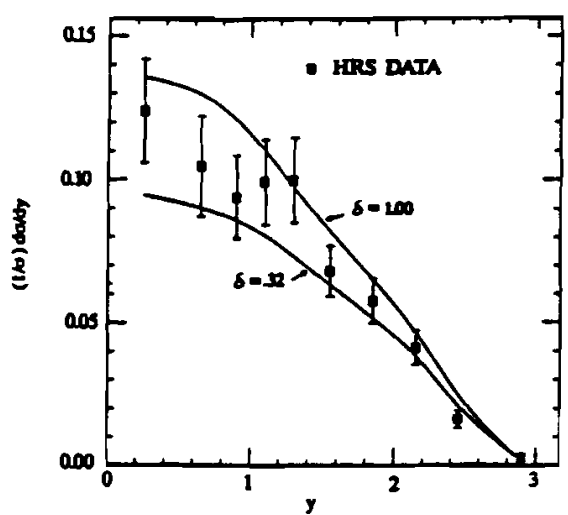

FIG. 6. Comparison of the mpidity distribution to predicted distributions for $\delta=0.32$ and 1.00 .

simulation (with $\delta$ varying between 0.1 and 1.4 ) to generate a new set of $\Lambda^{\prime} \mathrm{s}$. The $\delta$ value corresponding to the best fit is $0.59 \pm 0.10 \pm 0$. 18. (The deep minimum found in $\chi^{2}$ vs $\delta$ is a much stronger minimum than found in previous HRS publications [14].) The first error is statistical while the second is a systematic error based on the errors in $q q / q, s / d$, and $B\left(\Lambda_{c} \rightarrow \Lambda+X\right)$. The systematic error is consistent with Monte Carlo results obteined by varying each of these parameters independently. We note that $d \sigma / d y$ is much more sensitive than $d \sigma / d z$ to $\delta$, due at least in part to the additional information of the thrust axis contained in $y$. The curves shown in Figs. 3 and 5 are the inclusive cross sections with $\delta=0.59$, superimposed on the experimental cross sections. Figures 6 and 7 show, respectively, comparison of various $\delta$ values to the rapidity distribution and the contribution from $\Lambda_{c}$ decay. This value of $\delta$ is significantly larger than the default value of 0.32 in the standard Lund model $[4,5]$. The implication is that a larger fraction of the lambdas produced in inclusive electron-positron annihilations derive from the production of strange-diquark pairs than is postulated in the basic Lund model.

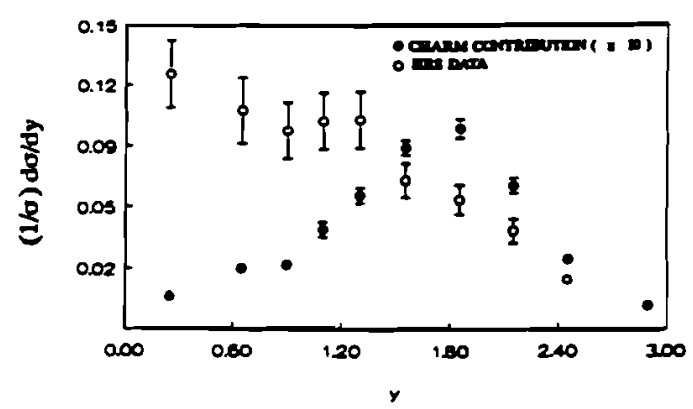

FIG. 7. Contribution to $\Lambda$ production from $\Lambda_{r}$ decay. (Charm contribution has been multiplied by a factor of 10. .)

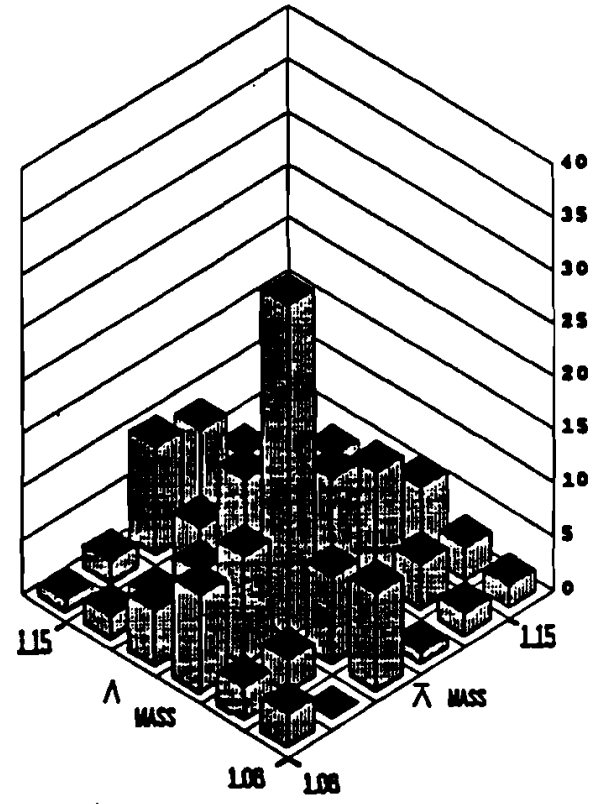

FIG. 8. Mass distribution of opposite-strangeness pairs.

\section{CORRELATIONS}

Additional insight into strange-baryon production processes can be gained by examining the rate at which $\Lambda \cdot \bar{\Lambda}$ pairs are produced, compared to the rate for the generation of single lambdas. This comparison provides a measure of local strangeness conservation and 2 test of the reasonableness of the proposed production processes.

We observed 212 candidates for $\Lambda-\bar{\Lambda}$. A threedimensional view of the $\Lambda \cdot \bar{\Lambda}$ distribution is shown in Fig. 8. We estimate that $68.2 \pm 27.5$ of these pairs are true sigmal events. The background subtraction is made using a detailed analysis of Monte Carlo events, with cross checks to ensure that the single-particle background spectra are well reproduced. We calculate a corresponding value of $\left(n_{A \bar{\Lambda}}\right)=0.046 \pm 0.020$, in agreement with previous experiments. The single-lambda inclusive production rate $\left(n_{\Lambda}\right)$ and the opposite-strangeness production rate $\left(n_{A}-\right)$ indicate $\lambda$ value of $0.51 \pm 0.23$, where

$$
\lambda=2 \frac{\left\langle n_{\Lambda \bar{A}}\right\rangle}{\left\langle n_{A}\right\rangle+\left\langle n_{\bar{\Lambda}}\right\rangle}
$$

indicating that half the time $\wedge \Lambda$ or $\bar{\Lambda}$ is produced, it is accompanied by its antiparticle. We have also examined our data for lambda pairs of the same strangeness and, employing the same background subtraction technique as above, find $\left(n_{A A}+\bar{\Lambda} \bar{\Lambda}\right)=0.009 \pm 0.028$. It is thus improbable that when a lambda of one strangeness is observed in an event another lambda of the same strangeness will also be present in that event. 
TABLE II. Comparison of A parameters.

\begin{tabular}{lccccc}
\hline \hline Experiment & Energy (GeV) & $n_{A}$ & $n_{A \pi}$ & $n_{A A+\pi}$ & $\delta$ \\
\hline TPC [10] & 29 & $0.211 \pm 0.017$ & $0.053 \pm 0.013$ & & \\
TASSO [9] & 35 & $0.218 \pm 0.023$ & $0.052 \pm 0.016$ & & \\
JADE [15] & 35 & $0.234 \pm 0.064$ & & $0.0052 \pm 0.0088$ & 0.48 \\
Mark II [16] & 29 & $0.213 \pm 0.022$ & & & \\
HRS-86 [3] & 29 & $0.220 \pm 0.023$ & $0.054 \pm 0.018$ & & $0.89 \pm 0.10 \pm 0.46$ \\
HRS & 29 & $0.182 \pm 0.020$ & $0.046 \pm 0.020$ & $0.009 \pm 0.028$ & $0.59 \pm 0.10 \pm 0.18$ \\
\hline
\end{tabular}

Value set.

\section{CONCLUSIONS}

We have reported the cross sections for inclusive lamb. da production in $e^{+} e^{-}$annihilations at $29 \mathrm{GeV}$, and have extracted the single-particle and pair-production rates. We have found $\left\langle n_{A}\right\rangle=0.182 \pm 0.020$, $\left\langle n_{A \bar{A}}\right\rangle=0.046 \pm 0.020$, and $\left\langle n_{M A+\bar{A}}\right\rangle=0.009 \pm 0.028$. Our cross-section measurements are consistent with the Lund model predictions, with $\delta=0.59 \pm 0.10 \pm 0.18$.

A comparison of the principal parameters extracted from recent lambda inclusive measurements is presented in Table II.

\section{ACENOWLEDGMENTS}

We wish to thank the stafis of SLAC and the . borating institutions whose efforts made this expern poscible. This work was supported in part by the Department of Energy under Contracts Nos. W. 31 ENG.38 (Argonne National Laboratory), DE.A1 765ER01112 (University of Michigan), DE.A1 76SF00098 (Lawrence Berkeley Laboratory), DE-A1 76ER01428 (Purdue University). and DE-Al 84ER40125 (Indiana University).

$2045(1990)$.

[1] HRS Collaboration, D. Bender et al., Phys. Rev: D 30 , S1S (1984).

[2] HRS Collaboration, M. Derrick at al., Phys. Rev. D 35, 2639 (1987).

[3] HRS Collaboration, P. Baringer et al, Phy. Rev. Letr. 56. 1346 (1986)

[4] T. Sjostrand. Comput. Phys. Commun. 27, 243 (1982).

[5] T. Sjostrand. Comput. Phys. Commun. 23, 229 (1983).

[6] H. Aihare et al. Report No. UCLA-HEP-90-002 (unpublished). A synopsis is published in K. Abe in Proceedings of the 2Sth Intermational Conference on High Energy Physic, Singapore. 1990, edited by K. K. Phus and Y. Yamaguchi (World Scientific, Singapore, 1991), p. 33.

[7] C. D. Buchanan and S.-B. Chun, Report No. UCLAHEP-90-003 (unpublished). A synopsis is published in Abe [6].

[8] HRS Collaboration, S. Abachi et al., Phys. Rev. D 41 ,
[9] TASSO Collaboration, W. Braunsehweis of al., 2 p C 45, 209 (1989).

[10] IPC Collaboration, H. Aihara et al., Phys. Rev. I rm 274 (11985).

[11] HRS Collaboration, M. Derrick at al., Phyz. Letl o 519 (1985).

[12] H. Yamamoto, in QCD and Beyond, Proceedins of Tweatieth Rencontre de Moriond. edited by 1 " Thanh Yan (Editions Frontiere, Gifsur-Yvette, 10091.

[13] K. Abe et al., Phys. Rev. D 33, 1 (1986).

[14] P. Buringer (private cormmuniction).

[15] JADE Collaboration, W. Bartel et al., Phys. Let * 325 (1981).

[16] Mart II Collaboration, C. de la Vaissiere et af $P$ Rev. Lett. S4, 2071 (1985). 


\title{
APPENDIX C
}

\section{Frequently Used Abbreviations}

\author{
ACD analog to digital converter \\ CC Central Calorimeter \\ CCCH Central Calorimeter Coarse Hadronic module \\ CCEM Central Calorimeter Electromagnetic module \\ CCFH Central Calorimeter Fine Hadronic module \\ CDC Central Drift Chamber
}

CCMG Central Calorimeter Massless Gap

DØ D-Zero; refers to the experiment, the collaboration responsible for the experiment, or (as D0), the interaction region for which the experiment was named

$\mathbf{E}_{\mathrm{T}}$ transverse energy

$E_{\mathrm{T}}$ missing transverse energy

EC End Calorimeter

ECEM End Calorimeter Electromagnetic module

ECIH End Calorimeter Inner Hadronic module

ECICH End Calorimeter Inner Coarse Hadronic module

ECIFH End Calorimeter Inner Fine Hadronic module

ECMH End Calorimeter Middle Hadronic module

ECMCH End Calorimeter Middle Coarse Hadronic module

ECMFH End Calorimeter Middle Fine Hadronic module

ECMH MG End Calorimeter Middle Hadronic Massless Gap

ECOH End Calorimeter Outer Hadronic module

ECOCH End Calorimeter Outer Coarse Hadronic module 
ECOH MG End Calorimeter Outer Hadronic Massless Gap

FDC Forward Drift Chambers

FNAL Fermi National Accelerator Laboratory

FP fluorinated polymer

ICD Intercryostat Detector

ICR intercryostat region

$\mathbf{L N}_{2}$ liquid nitrogen

LAr liquid argon

MG massless gap

MIP minimum ionizing particle

NWA Neutrino West Area

PE photoejectrons

PMMA polymethyl methacrylate

PMT photomultiplier tube

PS polysterene

PDT proportional drift tube

PWC proportional wire chamber

SAMUS Small Angle Muon System

SM The Standard Model

TRD Transition Radiation Detector

WAMUS Wide Angle Muon System

WLS wavelength shifting

VTX Vertex Chamber 


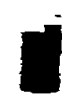

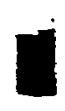

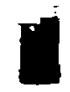

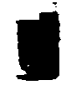

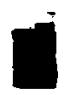

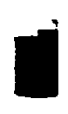

.

,

BIBLIOGRAPHY

.

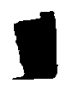

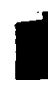

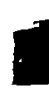

.

,

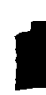

, 


\section{BIBLIOGRAPHY}

[1] Thanks go to Dr. Kaushik De for his leadership and all his help in the test beam project, particularly, the test beam electronics and the feedthrough port design.

[2] A. Salam. Weak and Electromagnetic Interactions. In N. Svartholm, editor, Elementary Particle Theory: Relativistic Groups and Analyticity, Proceedings of the Eighth Nobel Sympositm, page 367, 1968.

[3] S. Weinberg. A model of leptons. Phys. Rev. Lett., 19:1264, 1967.

[4] S. Glashow. The Vector Meson in Elementary Particle Decays. PhD thesis, Harvard University, 1959.

[5] M. Gell-Mann. A schematic model of baryons and mesons. Phys. Lett., 8:214, 1964.

[6] C. Quigg. Gauge Theories of the Strong, Weak, and Electromagnetic Interactions. Benjamin/Cummings., 1983.

[7] G. Kane. Modern Elementary Particle Physics. Addison-Wesley Publishing Co., 1987.

[8] D. Bender et al. Tests of QED at $29 \mathrm{GeV}$ center-of-mass energy. Phys. Rev., D30:515, 1984. The HRS Collaboration.

[9] T.L. Geld et al. Study of inclusive $\Lambda$ production in $e^{+} e^{-}$annihilations at $29 \mathrm{GeV}$. Phys. Rev., D45:3949, 1992.

[10] I would like to think Dr. Byron P. Roe for the use of his PAW-based Feynman diagram programs, which I used to produce all the Feynman diagrams in this thesis.

[11] P. Aurenche et al. Gluon content of the nucleon probed with real and virtual photons. Physical Review, D39:3275, 1989.

[12] P. Aurenche, R. Baier, and M .Fontannaz. Prompt photon production at colliders. Physical Review, D42:1440, 1990.

[13] P.D. Grannis et al. The DØ Design Report. Fermilab, unpublished., Nov., 1984.

[14] P.D. Grannis et al. The DØ Detector. To be submitted to NIM., 1993.

[15] M. Month and M. Dienes. Proceedings of the AIP Conference on Physics of Particle Accelerators. American Institute of Physics., 1989. See, for example, the references contained therein.

[16] A.R. Clark et al. The central tracking detectors for DØ. Nuclear Instruments and Methods in Physics Research, A279:243-248, 1989.

[17] S. Rajagopalan. The $d E / d x$ Capabilities of the $D \emptyset$ Tracking System. PhD thesis, Northwestern University, 1992. 
[18] J.F. Detœuf et al. Status of the Transition Radiation Detector for the D $\emptyset$ experiment. Nuclear Instruments and Methods in Physics Research, A279:310-316, 1989.

[19] J.F. Detœuf et al. The D $\emptyset$ Transition Radiation Detector. Nuclear Instruments and Methods in Physics Research, A265:157-166, 1988.

[20] F. Feinstein. Etude d'un Detecteur a Rayonnement de Transition pour l'Esperience $D \emptyset$ au FNAL. PhD thesis, A L'Universite de Paris-Sud, Centre d'Orsay, 1987.

[21] T. Behnke. The Central Drift Chamber for the $D \emptyset$ Experiment: Design, Construction and Test. PhD thesis, The State University of New York at Stony Brook, 1989.

[22] D. Pizzuto. DØ Central Tracking Chamber Performance Studies. PhD thesis, The State University of New York at Stony Brook, 1991.

[23] R. Avery et al. Results from a beam test of the $\mathrm{D} \emptyset$ forward drift chamber. IEEE Trans. Nucl. Science, NS-1234:1234, 1992.

[24] J. Bantly. The DØ Forward Drift Chamber Performance and Physics Capability in the 1990 FNAL Testbeam Run. PhD thesis, Northwestern University, 1992.

[25] M. Abolins et al. Hadron and electron response of uranium/liquid argon calorimeter modules for the $\mathrm{D} \emptyset$ detector. Nuclear Instruments and Methods in Physics Research, A280:36-44, 1989.

[26] R.D. Schamberger. D $\emptyset$ Calorimeter Electronics. In E. Borchi, T. Ferbel, D. Nygren, A. Penzo, and P.G. Rancoita, editors, Proceedings of the International Conference on Advanced Technology and Particle Physics, 1991.

[27] C. Brown et al. $\mathrm{D} \emptyset$ muon system with proportional drift tube chambers. Nuclear Instruments and Methods in Physics Research, A279:331-338, 1989.

[28] J.M. Butler et al. Study of fast gases, resolutions and contaminants in theD $\emptyset$ muon system. Nuclear Instruments and Methods in Physics Research, A290:122-130, 1990.

[29] D. Denisov. Small Angle Muon System (SAMUS) for the D $\emptyset$ Detector at Fermilab. DØ Note \#1367, unpublished., 1992.

[30] Y. Antipov et al. Drift tubes from the SAMUS muon spectrometer of the D $\emptyset$ detector at FNAL. DØ Note \#1278, unpublished., 1991.

[31] F. Nang and R. Partridge. Level 0 Detector Layout. D $\emptyset$ Note \#998, unpublished., 1990 .

[32] R. Partridge. The Level 0 Trigger. DØ Note \#1295, unpublished., 1991.

[33] R. Partridge. The Level 0 Trigger. DØ Note \#1218, unpublished., 1991.

[34] K. McFarland. Design and Testing of a Calibration System for the Level 0 Trigger at DØ. DØ Note \#840, unpublished., 1989.

[35] A. White. CC/EC Study Status Transparencies. DØ Note \#620, unpublished., 1987. 
[36] A. White. The Status of the Intercryostat Detector. Dø Note \#769, unpublished., 1988.

[37] A. Jonckheere. Simulating DO and Hermeticity Studies or How to Effect a Detector Deaign. DØ Note \#638, unpublished., 1987.

[38] M.G. Albrow et al. A uranium scintillator calorimeter with plastic-fibre readout. Nuclear Instruments and Methods in Physics Research, A256:23-37, 1987.

[39] K. De et al. Proposed Design for a Photomultiplier Tube Testing Facility. D $\emptyset$ Note \#925, unpublished., 1990.

[40] L. Oesch, K. De, and H. Neal. The Design, Construction and Testing of the DØ ICD Laser Calibration System. DØ Note \#1273, unpublished., 1993.

[41] Private communication with M. Marx, 11/89.

[42] G.R. Kalbfleisch. Possible Counter Array for Triggering a Bubble Chamber. In M. Derrick, editor, Proceedings of the International Conference on Bubble Chamber Technology, 1970.

[43] M. Atac et al. A Simple Method For Fusing Plastic Fibers. FNAL Preprint \#FN-537, 1990.

[44] I would like to thank my husband, Jon, for suggesting this wonderfully high-tech design to monitor the $\mathrm{LN}_{2}$ level.

[45] Albert L. Jew. Presentation of Research Project on the DZERO ICD Tiles. University of Michigan Honors Thesis project, unpublished. In addition, I would like to thank Albert for doing a special set of his Monte Carlo runs for me to study the effect of groove depth on light output in the test beam tiles., 1992.

[46] Many thanks to Bob Hirosky and Jerry Blazey for taking the time to do the contamination studies for the Testbeam ICD.

[47] R. Hirosky. Response of DØ U-LAr Calorimeters at Low Energies and the Effect of Oxygen Contamination. $\mathrm{PhD}$ thesis, The University of Rochester, 1993.

[48] G.C. Blazey. Monitoring Liquid Argon Purity at DØ. In D.F. Anderson, M. Derrick, H.E. Fisk, A. Para, and C.M. Sazama, editors, Proceedings of the First International Conference on Calorimetry in High Energy Physics, 1991.

[49] Thanks go to Dr. Robert C. Ball for his work in designing, building and installing the test beam calibration system.

[50] B. Daniels. Test Calorimeter Simulation Design. D $\emptyset$ Note \#1043, unpublished., 1990.

[51] The steel walls and cart were designed and provided by the Brookhaven National Laboratory $\mathrm{D} \emptyset$ group.

[52] The foam excluder was designed and provided by the University of Rochester $D \emptyset$ group. 
[53] P. Bhat. TB1990 Beamline Operation, Performance and Studies. DØ Note \#1076, unpublished., 1990.

[54] T.L. Geld, K. De, and J.P. Borders. Test Beam ICD DST format. DØ Note \#1271, unpublished., 1992.

[55] L. Landau. In Russian. Journal of Experimental Physics (USSR), 8:201, 1944.

[56] F. James and M. Roos. MNNUTT, Function Minimization and Error Analysis, Release 89.12. CERN Program Library, D506, unpublished., 1989.

[57] W.G.D. Dharma et al. DØ End Calorimeter Response to Muons and $\frac{4}{e}$ Ratio - Test Beam Load 1. DØ Note \#1351, unpublished., 1992.

[58] Calorimeter $d E / d x$ values determined by Dan Owen.

[59] J.P. Borders. Calibration of the DZERO Hadronic Response Using Test Beam Data. DØ Note \#1502., 1993.

[60] S. Peryshkin. Short Explanation of how the Sampling Weights for CCEM were chosen. DØ Note \#1498, unpublished., 1992.

[61] A.L. Spadafora. Test beam Results from the D $\emptyset$ Endcap Electromagnetic Calorimeter Module. In D.F. Anderson, M. Derrick, H.E. Fisk, A. Para, and C.M. Sazama, editors, Proceedings of the First International Conference on Calorimetry in High Energy Physics, 1991.

[62] R. Brun et al. GEANT3.14 Users Guide. CERN Preprint, unpublished., 1989.

[63] T.C. Heuring. Electrons in the DØ Central Calorimeter: A Comparison of Testbeam Data and Monte Carlo Simulation. PhD thesis, The State University of New York at Stony Brook, 1993.

[64] R. Wigmans. On the energy resolution of uranium and other hadron calorimeters. Nuclear Instruments and Methods in Physics Research, A259:389-429, 1987.

[65] K. De. A Monte Carlo Study of Hadronic Energy Corrections in the ICD. D $\emptyset$ Note \#1017, unpublished., 1990.

[66] R.J. Madaras. Highlights from DØ. In C.H. Albright, P.H. Kasper, R. Raja, and J. Yoh, editors, Proceedings of the 7th Meeting of the American Physical Society Division of Particles and Fields., page 19, 1993.

[67] F. Abe et al. Measurement of the W-boson mass in 1.8-TeV p p collisions. Phys. Rev., D43:43, 1991.

[68] R.V. Astur. Inclusive Jet Cross Sections at the DØ Detector. In C.H. Albright, P.H. Kasper, R. Raja, and J. Yoh, editors, Proceedings of the 7th Meeting of the American Physical Society Division of Particles and Fields., page 993, 1993.

[69] Thanks go to Dr. Kaushik De for these analyses and figures. 